\title{
Synaptic Ultrastructure and \\ Regulation of Synaptic Transmission in Caenorhabditis elegans
}

\author{
Dissertation
}

\author{
for the award of the degree \\ "Doctor rerum naturalium" \\ of the Georg-August-Universität Göttingen
}

submitted by

Maike Kittelmann

born in

Erfurt, Germany

Göttingen 2012 


\section{Thesis Committee:}

Dr. Stefan Eimer ( $1^{\text {st }}$ reviewer, advisor $)$

Dept. of Molecular Neurogenetics, European Neuroscience Institute Göttingen

Prof. Dr. André Fiala ( $2^{\text {nd }}$ reviewer $)$

University of Göttingen, Dept. of Molecular Neurobiology of Behaviour

Prof. Dr. Martin Göpfert

University of Göttingen, Dept. of Cellular Neurobiology

Date of the oral examination:

21.06.2012 
Herewith I declare, that I prepared the dissertation "Synaptic Ultrastructure and Regulation of Synaptic Transmission in Caenorhabditis elegans" on my own and with no other sources and aids than quoted.

Göttingen, May $9^{\text {th }}, 2012$

Maike Kittelmann 
For my family 


\section{Acknowledgement}

I want to thank Dr. Stefan Eimer for his supervision and the opportunity to work on this exciting project in his lab.

A big thank you goes to the people of the Eimer lab, especially Christoph, Jan, our external Caro, Mandy, Nora, Nikhil, Ling, Janny, Marija, Christian, Sabine and Katrin. You were very supportive and created a nice working atmosphere during the last three years. Special thanks to Mandy, Nora and Caro for helpful comments on this thesis! Furthermore I want to thank Sabine and Katrin for running the lab and providing technical help when needed.

I also want to thank Ali Pilewa and Matthias Weyl for outstanding IT assistance, Heiko Röhse for taking care of the Confocal microscope and its users and the ENI staff for creating this work nice environment.

I also want to thank my thesis committee members Prof. Fiala and Prof. Göpfert for their time and valuable input during this thesis.

Spending 10 months abroad at the University of California, San Diego was a very important experience on a professional as well as on a personal level. This adventure would not have been possible without the support of the Education Abroad Program Göttingen and the Fulbright Foundation, which I greatly thank.

A special thank you goes to Prof. Yishi Jin and Prof. Andrew Chisholm and their lab who took me in for a year as well as Prof. Ellisman and his lab who patiently taught me EM tomography at NCMIR, UCSD. Thank you Alex Goncharov for sharing your EM workspace and your insights into worm ultrastructure with me!

Gary and Aline Christiansen are the people who made me feel at home in San Diego and I can't thank them enough for opening their home and family to me and getting me started with the everyday commodities.

I want to thank my collaborators Prof. Yishi Jin, Prof. Hidenori Taru and Prof. Janet Richmond for their input and helpful discussions and comments on the project and this thesis. 
I am grateful to be a member of the GGNB Sensory and Motor Neuroscience program. I want to thank GGNB not only for supporting me financially with a Junior Group Stipend, a Bridging Fund and Travel Grants, but also providing me with a variety of method courses and opportunities to meet with other scientists. Thanks also to Tobias Moser, the other students of the SMN program and the organizing team in the background for fun and exciting retreats!

A big thank you also goes to the GGNB administration for taking care of all the tedious bureaucracy that is involved in graduating!

Electron microscopists are a very special kind of people and I was lucky enough to be office mates with three of them over the years. They all enriched not just my everyday life, but also shared much of their knowledge and experience.

Thank you Caro (Cora) for the never-ending supply of Drei??? audio books, chocolate and Gunnar stories!

Thank you Jan (Jens) for patiently supervising me, singing EAV for me and making me laugh until tears were running.

Thank you Christoph (Carsten) for keeping me updated on the world news, amusing me with your paranoia, eating all my candy ;0) and especially for putting up with me while writing this thesis.

The time in Göttingen will always be connected to the friends that I first made during the orientation way back in 2003. Sabine, Eva, Henning, Micha, Kristin, Stephan, Marcus and Basti, thank you for the amazing parties, great times and many memories!

Many thanks to my parents and my immediate and extended family for their infinite love and support. You may never completely understand what I do, but you never get tired trying. I am very lucky!

Last but not least I want to thank my husband for his unconditional love, his support in anything I do, the countless times I could lay back and rely on him, the trust and strength to let me leave for 10 months to the U.S. and the way he makes me feel save and at home when he is with me. 



\section{Table of Contents}

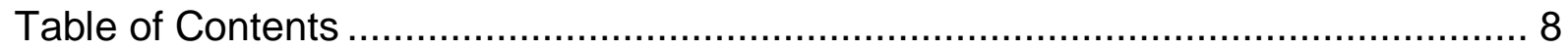

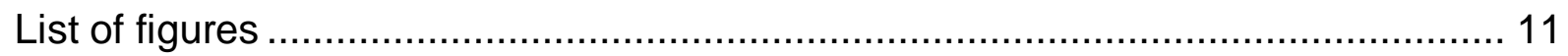

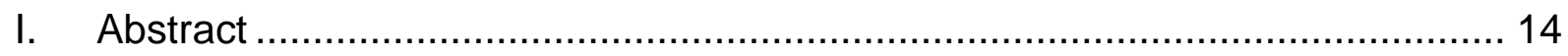

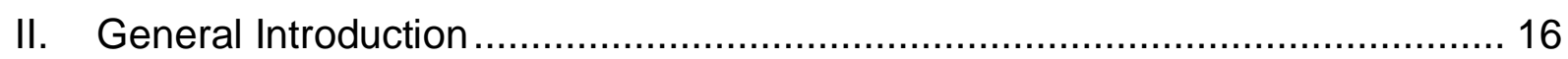

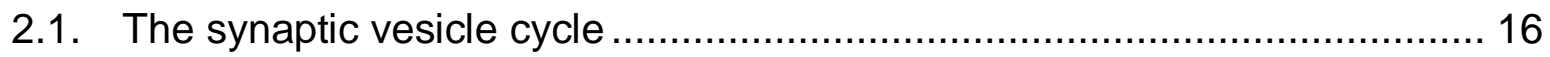

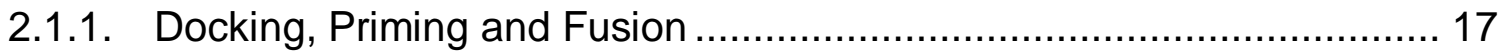

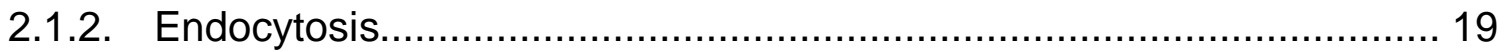

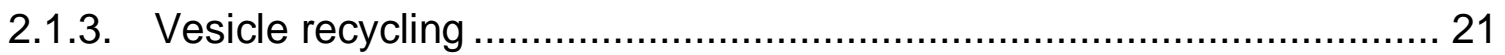

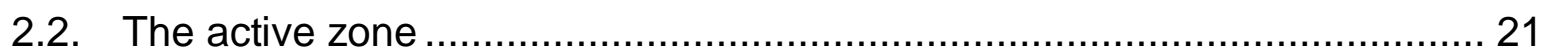

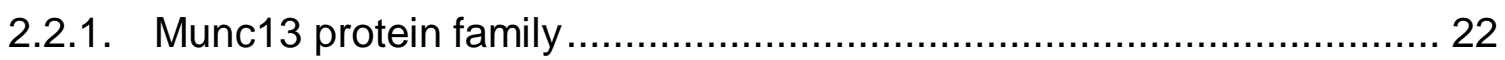

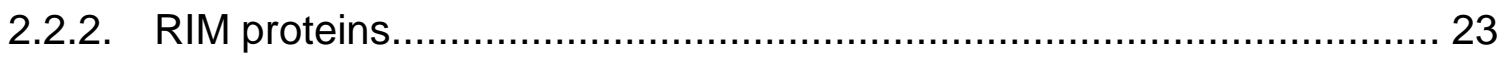

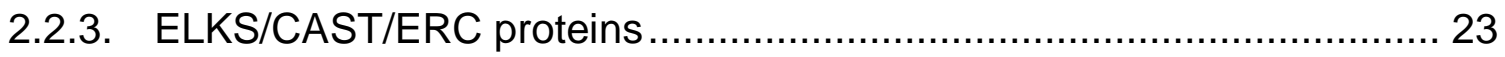

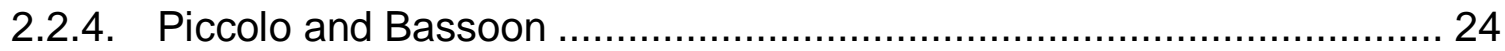

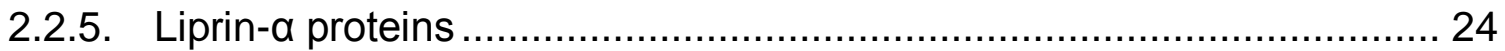

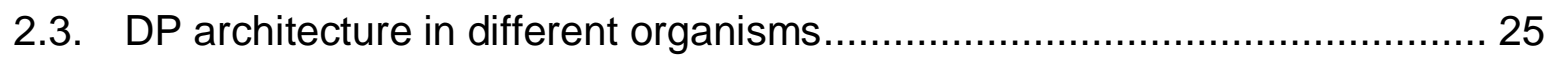

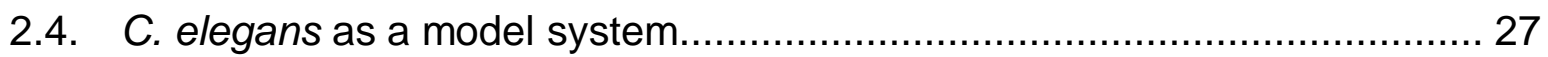

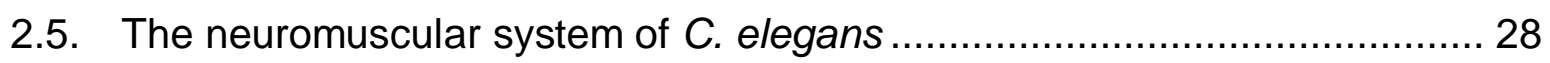

2.6. High-pressure-freezing and freeze substitution for electron microscopy .... 34

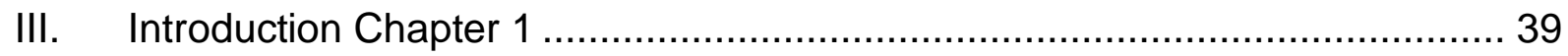

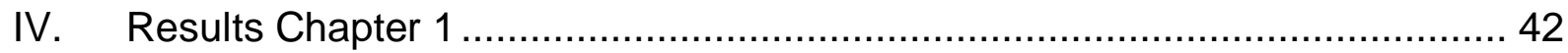

4.1. 3D reconstructions reveal the complex structure of presynaptic dense

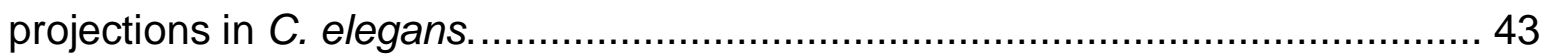

4.2. The active zone protein SYD-2 regulates the size of DPs and affects proper

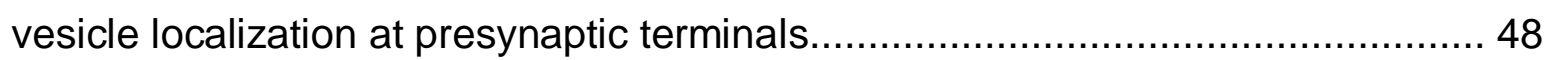

4.3. Synaptic transmission is affected in syd-2 mutant animals....................... 58

4.4. The ability of SYD-2 to promote the formation of longer DPs depends on

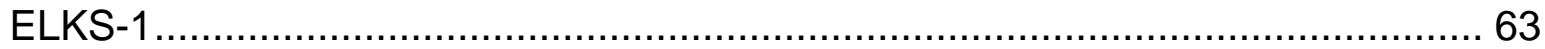

4.5. Loss of the negative regulator RSY-1 enhances elongation of DPs in syd-2

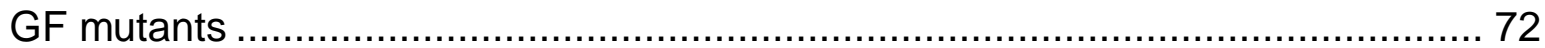

4.6. The size of NMJ DPs is regulated by counteracting regulators ................. 75

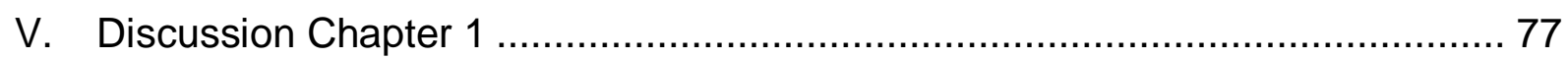


5.1. A branched DP structure can provide a microenvironment for vesicle fusion. .

5.2. SYD-2 and RSY-1 are counteracting proteins that define NMJ DP size..... 80

5.2.1. SYD-2 determines the length of NMJ DPs ............................... 80

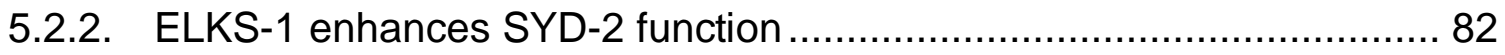

5.2.3. RSY-1 counteracts SYD-2 function in defining DP size ..................... 83

5.2.4. Interaction of SYD-2 with kinesin UNC-104 as mechanism to alter DP size

5.2.5. Synaptic plasticity via regulation of DP size ............................... 85

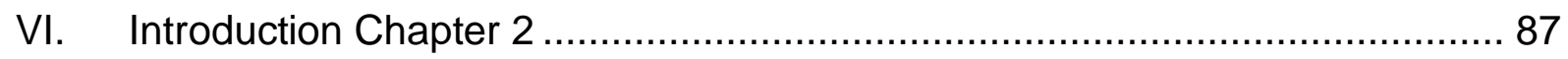

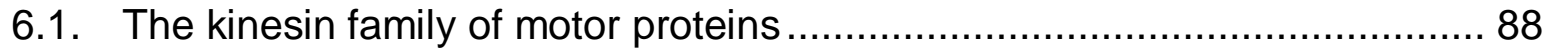

6.2. Kinesin cargo recognition and unloading ...................................... 89

6.3. UNC-104 and its function in synaptogenesis .................................... 90

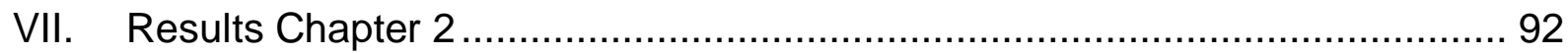

7.1. Synaptic vesicles mislocalize to cell bodies and dendrites in unc-104 mutants

7.2. Active zone proteins localize to axons and dendrites in unc-104 mutants... 96

7.3. SVs and DCVs are absent from synapses in dorsal cord motor neurons... 98

7.4. DCV-like vesicle accumulation and ectopic synapses formation in unc-104

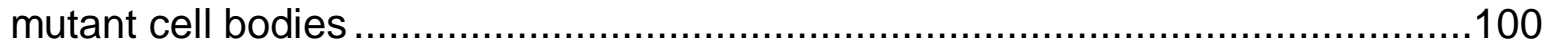

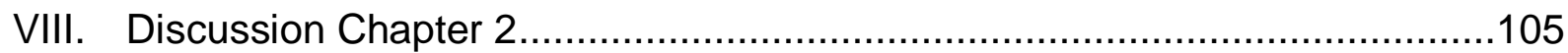

8.1. UNC-104 specifically transports large DCV-like SV precursors ................106

8.2. Active zone components are transported differently than SV precursors and

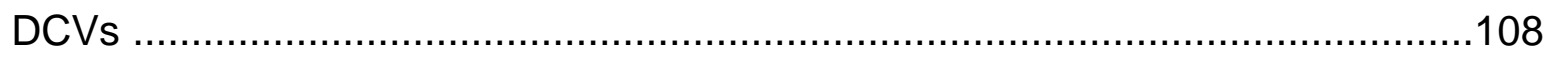

8.3. Ectopic synapses are formed in unc-104 mutant cell bodies....................111

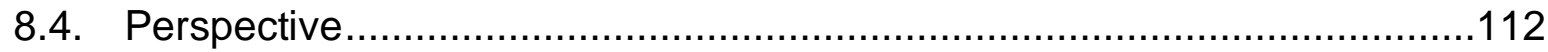

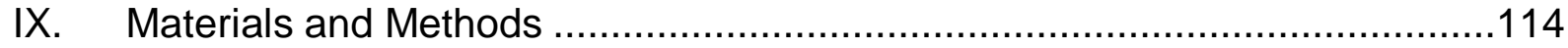

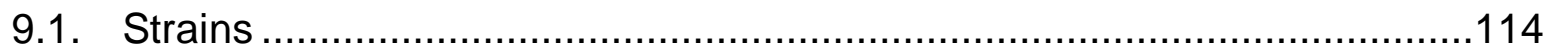

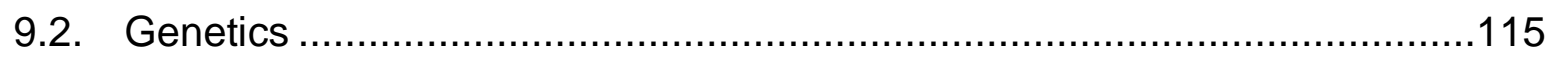

9.3. High-pressure-freezing / Freeze substitution ................................116

9.4. Production of formvar coated grids...........................................116

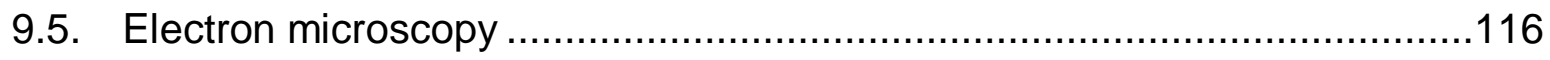

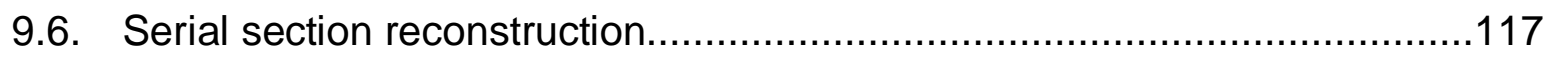

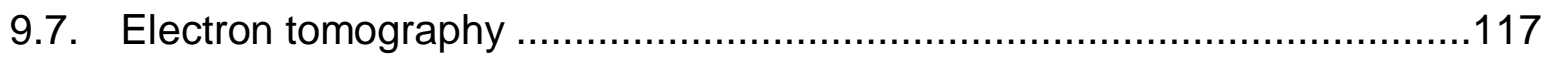




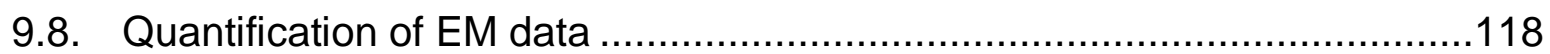

9.9. Fluorescence microscopy and quantification .................................118

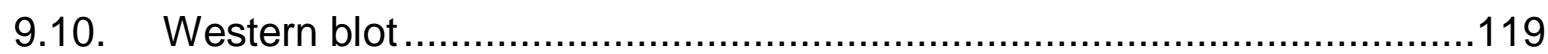

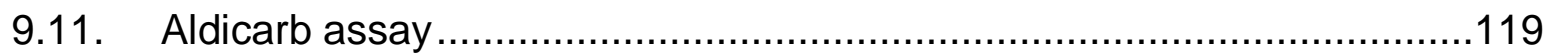

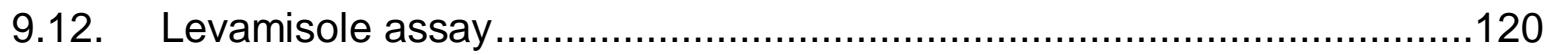

9.13. PCR mutagenesis and cloning ..................................................

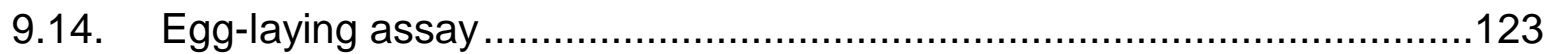

9.15. Biochemistry (performed by collaborator Hidenori Taru) .....................123

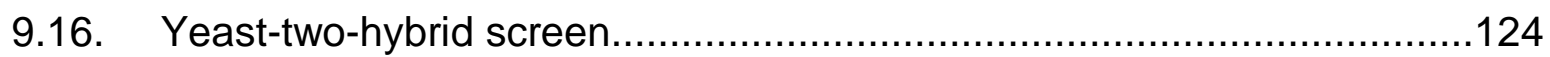

9.17. Electrophysiology (performed by collaborator Janet Richmond) ............124

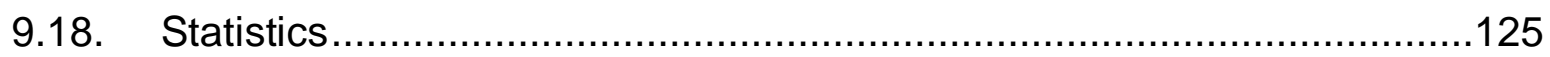

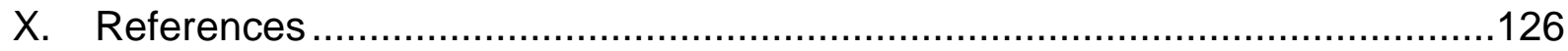

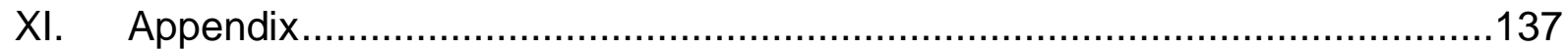

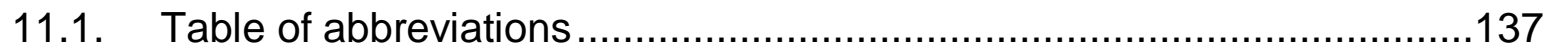

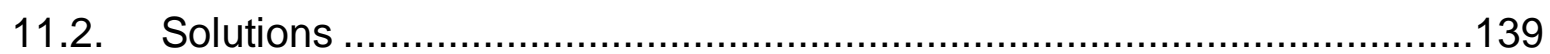

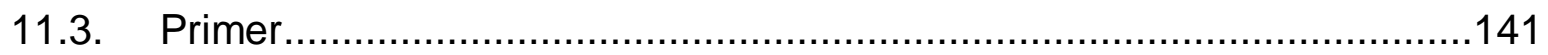

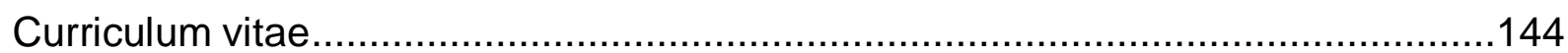




\section{List of figures}

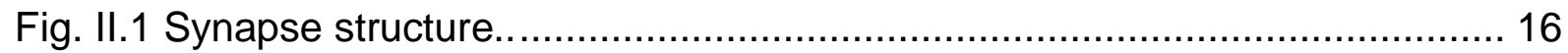

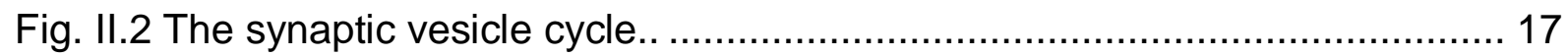

Fig. II.3 Formation of the SNARE-complex between vesicle and cell membrane.... 19

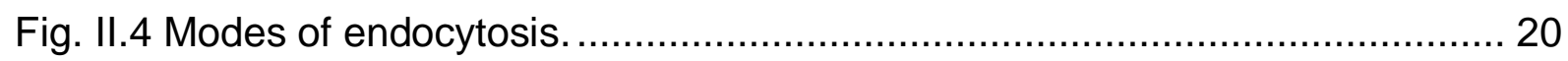

Fig. II.5 Protein structure and domains of CAZ proteins................................ 21

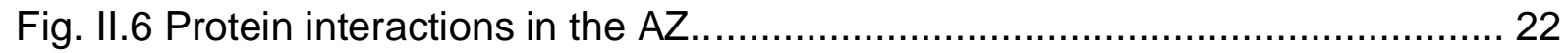

Fig. II.7 DP architecture in different organisms and synapse types.................... 26

Fig. II.8 The nervous system of C. elegans. .............................................. 28

Fig. II.9 Muscle arms extend from body wall muscles to form neuromuscular

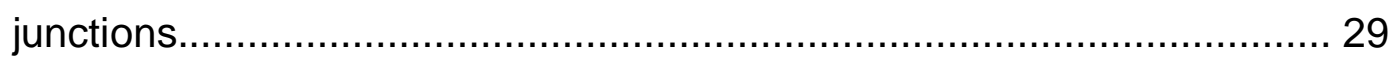

Fig. II.10 Types of motor neurons in C. elegans....................................... 30

Fig. II.11 Alternating contraction of dorsal and ventral body wall muscles............... 31

Fig. II.12 Differentiation of GABAergic and cholinergic synapses in C. elegans....... 32

Fig. II.13 Neuronal wiring to elicit backward locomotion upon anterior and posterior

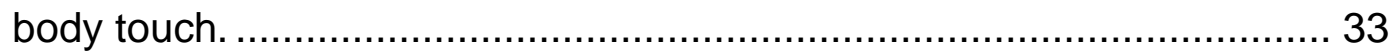

Fig. II.14 Cryofixation enhances ultrastructural preservation in C. elegans............. 36

Fig. II.15 Workflow from high-pressure-freezing to imaging........................... 37

Fig. II.16 Electron microscopy tomography............................................ 38

Fig. III.1 Hierarchical assembly of the active zones in C. elegans...................... 40

Fig. IV.1 Synapse morphology at neuromuscular junctions. .............................. 43

Fig. IV.2 Dense projections of $C$. elegans NMJs often branch............................ 44

Fig. IV.3 Fibrous extensions extend from the DP contacting adjacent SVs. ........... 44

Fig. IV.4 Branched morphology of the NMJ DP was verified by longitudinal thin sections and tomography...................................................... 45

Fig. IV.5 Architecture of GABAergic and cholinergic synapse DPs are similar....... 47

Fig. IV.6 Neuron-neuron synapse DPs have a similar geometric organization as NMJ

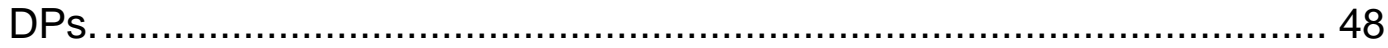

Fig. IV.7 syd-2 gene and SYD-2 protein structure. ...................................... 49

Fig. IV.8 Western blot analysis following SDS-PAGE of worm lysates. ................. 50

Fig. IV.9 DP length of NMJs is affected in syd-2 mutants. ............................ 52

Fig. IV.10 3D reconstructions of NMJ DPs indicate the preservation of branched

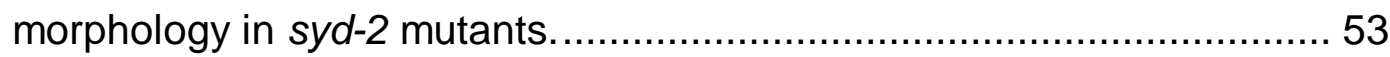


Fig. IV.11 3D Reconstructions of GABA- and cholinergic motor neurons in the ventral nerve cord of wild type and syd-2 mutants................................. 54

Fig. IV.12 Less SVs and DCVs are localized at NMJ DPs in syd-2 LF mutants. ..... 55

Fig. IV.13 Synaptic vesicle localization is altered at cholinergic NMJs in syd-2 LF

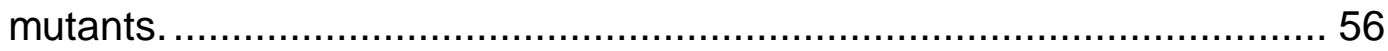

Fig. IV.14 Synaptic vesicle localization is altered at GABAergic NMJs in syd-2 LF mutants.

Fig. IV.15 Localization of AZ marker RIM-1/UNC-10 at NMJs is normal in syd-2 mutants.

Fig. IV.16 Reduced numbers of SVs are docked to the AZ membrane in syd-2 LF mutants. 59

Fig. IV.17 Evoked response is reduced in syd-2 LF and syd-2 super-GF mutants. .. 60

Fig. IV.18 syd-2 LF mutants are egg-laying defective.

Fig. IV.19 Aldicarb sensitivity is altered in syd-2 mutants. 62

Fig. IV.20 Levamisole sensitivity is normal in syd-2 mutants except for syd-2(ok217) LF. 63

Fig. IV.21 elks-1 gene and ELKS-1 protein structure. 64

Fig. IV.22 Axon and synapse morphology is unaltered in elks-1 LF mutants. 65

Fig. IV.23 Normal SV docking and vesicle clustering in elks-1 LF mutants. 66

Fig. IV.24 Normal evoked response in elks-1 LF mutants. 67

Fig. IV.25 The amino acid exchange R184C in SYD-2 GF protein leads to enhanced interaction with ELKS-1.

Fig. IV.26 SYD-2 LH1 with R184C amino acid exchange shows enhanced binding to ELKS-1. 69

Fig. IV.27 The highly conserved LH1 domain in the SYD-2 N-terminus oligomerizes more easily when the R184C substitution is present. 69

Fig. IV.28 Double syd-2; elks-1 LF mutants are similar to syd-2 single LF mutants. 70 Fig. IV.29 ELKS-1 is required in the syd-2 GF mutant to form elongated NMJ DPs. 71 Fig. IV.30 Evoked response is not altered in elks-1(js816); syd-2(ju487) double mutants.

Fig. IV.31 Loss of the negative regulator rsy-1 enhances the formation of elongated NMJ DPs in syd-2 GF mutants.

Fig. IV.32 3D Reconstructions of GABAergic and cholinergic motor neurons in the ventral cord of syd-2 GF; rsy-1 LF double mutants. 
Fig. IV.33 LF mutations in genes known to encode AZ proteins do not affect NMJ DP

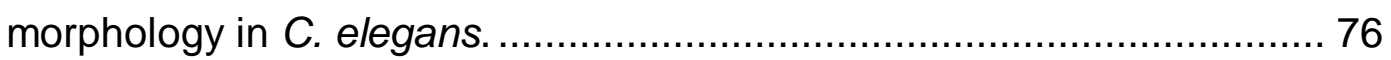

Fig. V.1 Bay structure in C. elegans DPs................................................ 78

Fig. V.2 Tangential view of the presynaptic NMJ dense T-bar base in the larvae of the flesh flie Sarcophaga bullata.................................................. 78

Fig. V.3 Positive and negative regulators control DP size. .................................. 86

Fig. VI.1 Intracellular transport by different kinesin motors in neurons. .................. 87

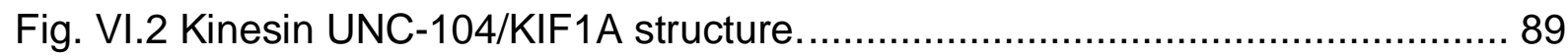

Fig. VII.1 UNC-104 protein domains and mutation sites................................ 92

Fig. VII.2 SV marker expression in wild type and unc-104 mutants..................... 94

Fig. VII.3 DCV marker expression in wild type and unc-104 mutants. .................... 95

Fig. VII.4 AZ marker expression in wild type and unc-104 mutants. ...................... 97

Fig. VII.5 Vesicle transport to synaptic sites is severely disturbed in unc-104 mutants.

Fig. VII.6 Accumulation of DCV-like vesicles and ectopic synapse formation in unc104 mutant cell bodies. 101

Fig. VII.7 DCV-like vesicles in the cell bodies of unc-104 mutants are larger than wild type synaptic SCVs. .102

Fig. VII.8 Clear core vesicles at cell body DPs in unc-104 are smaller than wild type SVs. .103

Fig. VII.9 Muscle arms contact cell bodies in unc-104 mutants. 104 


\section{Abstract}

The triggered release of synaptic vesicles (SVs) is tightly regulated in time and space by an elaborate network of proteins forming the active zone (AZ). When visualized by electron microscopy (EM), a morphological hallmark of the presynaptic $A Z$ is an electron dense projection (DP) situated at the center of the $A Z$ and extending into the cytoplasm. DPs are surrounded by a cluster of clear core SVs and have been proposed to play an important role in organizing and regulating SV release. However, our knowledge about its molecular composition, assembly mechanisms, and highresolution 3D structure are limited. The neuromuscular junction (NMJ) of the nematode Caenorhabditis elegans provides an easily accessible model system to study AZ assembly, structure and function in regulated synaptic transmission. By using high-pressure-freeze (HPF) and freeze substitution (FS) EM in combination with tomography, I could show that $C$. elegans DPs are highly structured. DPs at cholinergic and GABAergic NMJs are branched, forming bay-like slots in which SV can usually be found docked to the AZ membrane. These bays thus may correspond to SV release sites. Large neuron-neuron synapse DPs appear to be polymers of the smaller NMJ DP unit with the same geometric arrangement of branch points and bays, indicating that DPs at functionally distinct synapses in C. elegans employ a common principle of structural assembly.

To gain insights into the mechanisms that control synapse assembly, we analyzed the DP morphology in mutants lacking AZ proteins by HPF EM. Previous studies have proposed the $C$. elegans Liprin- $\alpha$ homolog synapse defective 2 (SYD-2) to be a key regulator in synapse formation by recruiting other synaptic proteins to nascent release sites. I demonstrate that SYD-2 together with a second AZ protein, ELKS-1, determines DP size in C. elegans NMJs. Whereas loss of SYD-2 results in significantly reduced DP size and inefficient vesicle recruitment, a gain-of-function (GF) mutation leads to the formation of elongated DPs. However, the ability of SYD-2 GF to promote DP elongation strictly depends on the presence of ELKS-1. A negative regulator of synapse formation, RSY-1, has recently been discovered to impair SYD2 functionality. In this study I provide evidence indicating that RSY-1 acts as a counteracting negative regulator of DP size. I therefore implicate SYD-2/Liprin- $\alpha$ in the dynamic polymerization of DPs in cooperation with other regulators, rather than in the generation of DPs per se. 
To gain deeper insight into how axonal transport contributes to synapse assembly, I analyzed mutants with defective motor proteins known to be involved in synaptic protein transport. Kinesin-3 UNC-104 has been identified as a neuron-specific anterograde motor protein required for the transport of SVs and DCVs to synaptic sites. Loss of UNC-104 results in the accumulation of vesicles in the cell bodies. To understand if and how this transport defect affects $A Z$ assembly, I analyzed $C$. elegans unc-104 mutants by confocal and electron microscopy. My initial analysis provides further evidence that the UNC-104 cargo binding domain is responsible for the attachment of SV precursors and DCVs to UNC-104. The formation of AZ DPs is, however, only moderately affected in unc-104 mutants, implying that organelles associated with $A Z$ components may utilize alternative transport mechanisms. For the first time, I report the formation of ectopic, but functional, synapses in unc-104 mutant cell bodies. Furthermore, the vesicles accumulating in the cell bodies were characterizes as DCV-like vesicles distinct from synaptic DCVs in wild type. We suggest that these DCV-like vesicles are SV precursors that transport SV proteins to AZs, where mature SVs can then be assembled by local endocytosis. 


\section{General Introduction}

Nervous systems range from very simple neuronal networks to the highly complex human brain with billions of neurons. As the word "system" implies, neurons interact with each other to realize the challenges of the nervous system to control and coordinate all vital functions in the organism. The structural elements of neurons that enable their communication with each other or with their specific target cells are called synapses (Foster and Sherrington, 1897). The most common chemical synapse typically consist of three components (Peters and Palay, 1996): (1) the presynaptic terminal where the incoming electrical signal is transformed into a chemical signal in form of neurotransmitter released from synaptic vesicles (SVs) via exocytosis in the active zone (AZ); (2) the synaptic cleft into which SVs secrete their neurotransmitter and (3) the postsynaptic terminal with specific neurotransmitter receptors where the chemical information is retransformed into an electrical signal via opening of ion channels (Fig. II.2).

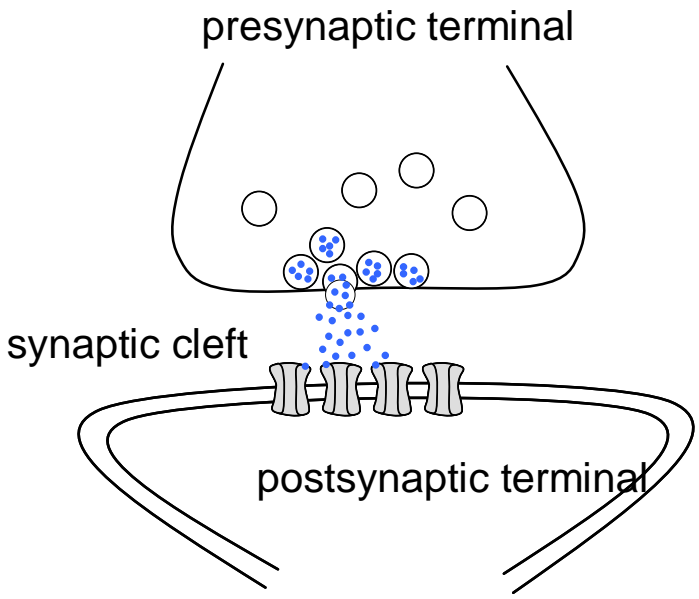

Fig. II.1 Synapse structure. The asymmetrical architecture of a chemical synapse consists of a presynaptic terminal where the stimulus arrives and triggers SVs to fuse with the plasma membrane. Released neurotransmitters diffuse into the synaptic cleft towards the receptor field of the postsynaptic terminal. Binding of neurotransmitters to the ligand-gated ion channel receptors results in ion influx and an evoked response in the postsynaptic target cell.

To allow efficient and reliable synaptic transmission, exocytosis and subsequent endocytosis of SVs have to be tightly regulated in time and space (Wojcik and Brose, 2007).

\subsection{The synaptic vesicle cycle}

The synaptic release machinery has been under intense investigation during the last decades. A number of proteins have been reported to be crucial for regulating the 
different steps of the vesicle cycle: docking, priming, fusion, local endocytosis and recycling (Fig. II.2) (reviewed in Sudhof, 2004, Richmond, 2005).

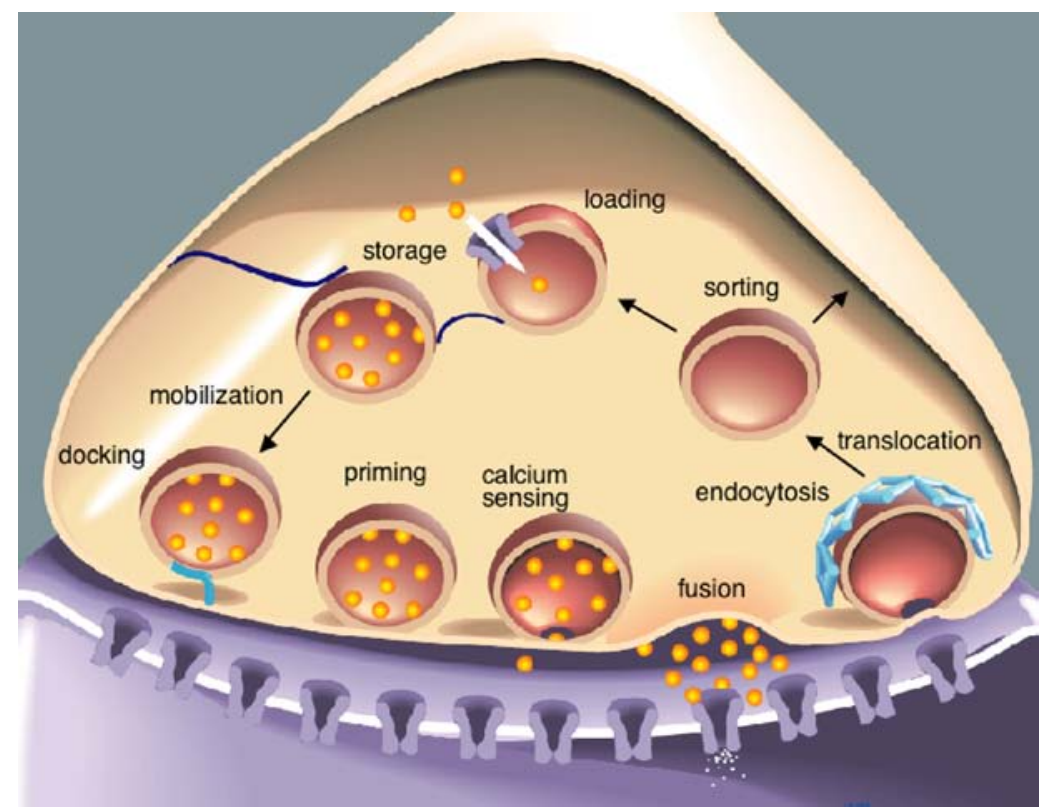

Fig. II.2 The synaptic vesicle cycle. SVs are filled with neurotransmitters and transported to the presynaptic $A Z$ membrane to which a subset of the vesicles docks. These vesicles become fusion-competent during the priming step. Upon arrival of an action potential, calcium levels rise, calcium binds to calcium sensors and thus triggers vesicle fusion with the presynaptic membrane. Neurotransmitters are released into the synaptic cleft where they bind to postsynaptic receptors and induce an evoked response. Vesicle membrane and proteins from the synaptic cleft are retrieved via clathrin-mediated endocytosis. Taken from (Richmond, 2005). This work is licensed under the Creative Commons Attribution 3.0 Unported License. To view a copy of this license, visit http://creativecommons.org/licenses/by/3.0/ or send a letter to Creative Commons, 444 Castro Street, Suite 900, Mountain View, California, 94041, USA.

\subsubsection{Docking, Priming and Fusion}

SVs are mobilized into the proximity of the AZ where they first dock to the presynaptic membrane and are then primed into a "readily releasable" state. The differentiation between these two conditions has been coined by electrophysiological properties. While primed vesicles are rapidly released upon arrival of an action potential or calcium influx, docked vesicles although close to the presynaptic membrane, are only released upon sustained stimulation, for example via sucrose (reviewed in Schikorski and Stevens, 2001, Rettig and Neher, 2002). In electron micrographs of conventionally fixed samples it has not been possible to clearly differentiate these two vesicle "pools" because both sit directly on or very close to the presynaptic membrane. Today, new preparation and imaging techniques as highpressure-freezing (HPF) and electron microscopy (EM) tomography (refer to 2.6) may help to identify docked versus primed vesicles (reviewed in McDonald and Auer, 
2006, Siksou et al., 2009). The interaction of the SV associated GTPase RAB-3 with the AZ proteins RIM (Rab3-interacting molecule; (Wang et al., 1997)), which in turn binds to Munc13 and calcium channels (Coppola et al., 2001) may resemble a molecular mechanism for docking SVs to the AZ in close vicinity of the calcium influx.

Priming of docked vesicles is characterized by partial formation of the SNARE complex between $\mathrm{v}$ - and t-SNARES localized in the vesicle and target plasma membrane. SNARE proteins play a central role in membrane fusion processes (reviewed in Chen and Scheller, 2001, Jahn et al., 2003). They were identified in an affinity screen as REceptors for N-ethylmaleimide-sensitive fusion proteins (NSF) and soluble NSF attachment proteins (SNAPS), both of which are components of the intracellular membrane fusion apparatus (Söllner et al., 1993). SNARES are small, membrane-associated proteins with a C-terminal transmembrane domain, a characteristic SNARE motif of 60-70 highly conserved amino acids and an N-terminal domain (reviewed in Hong, 2005, Jahn and Scheller, 2006, Kloepper et al., 2007). The initial differentiation of $v$-SNARES versus t-SNARES is now replaced by a more recent classification that differentiates between $\mathrm{Q}$ (glutamine) and $\mathrm{R}$ (arginine) SNARES, depending in the conserved amino acid in their SNARE motif contributing to the SNARE 0 layer of the SNARE complex (Fasshauer et al., 1998). A set of both types specifically bind to each other via their $\alpha$-helical SNARE motifs to form the SNARE complex and thus ensure the correct compartments to fuse. The SNARE complex (also called SNAREpin) is assembled by rapid N- to C-terminal zipping of the four helices into a helix bundle. This process brings the two membranes into close proximity and thus catalyzes membrane fusion (Pobbati et al., 2006). The SNARE complex has been shown to be minimally required for membrane fusion in vitro (Weber et al., 1998). In synapses the R-SNARE synaptobrevin is localized in the membrane of SVs while the Qa-SNARE syntaxin-1 and Qbc SNARE SNAP-25 (synaptosome-associated protein of $25 \mathrm{kDa}$ ) are localized in the presynaptic membrane (Sutton et al., 1998) (Fig. II.3). 


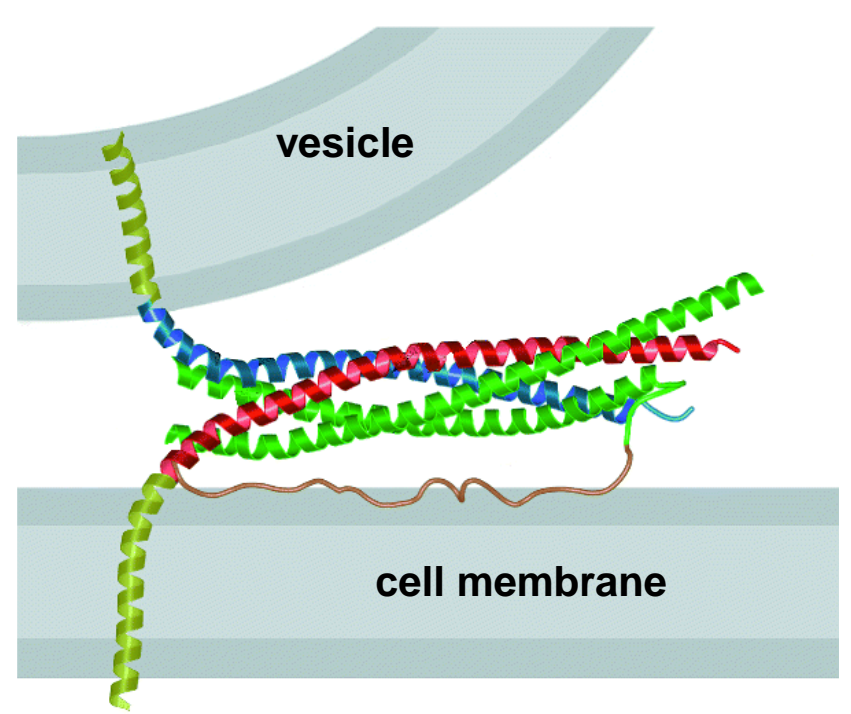

Fig. II.3 Formation of the SNARE-complex between vesicle and cell membrane. Syntaxin (red) and SNAP-25 (green) are anchored in the presynaptic cell membrane whereas synaptobrevin is associated with the vesicle membrane (blue). A four helix bundle is formed by the SNARE motifs from syntaxin and synaptobrevin as well as the two SNARE motifs provided by SNAP-25. Modified with permission from Macmillan Publishers Ltd: Nature (Sutton et al., 1998), copyright 1998.

The partially assembled SNARE complex of primed SVs keeps the two membranes about 3-4 $\mathrm{nm}$ apart and is held at this intermediate state by grappling proteins such as complexin (reviewed in Sudhof and Rothman, 2009). These vesicles can rapidly fuse upon stimulation and are therefore considered "readily releasable". The vesicleassociated calcium sensor synaptotagmin has been implicated in SV release (Perin et al., 1990, Brose et al., 1992, Fernandez-Chacon et al., 2001). Binding of calcium to the two $\mathrm{C} 2$ domains of synaptotagmin is thought to trigger completion of SNARE complex formation and binding of phospholipids in the plasma membrane (Fernandez et al., 2001). Insertion of four membrane-binding loops from the C2 domains results in membrane curvature, therefore supporting the hemifusion between the vesicle and plasma membrane (Martens et al., 2007). Upon formation of a fusion pore neurotransmitters are secreted into the synaptic cleft and diffuse towards the postsynaptic side and its receptors. These ligand-gated ion channels open upon binding of specific neurotransmitters leading to a hyper- or depolarization of the postsynaptic cell, depending on the type of ion channel (Stroud et al., 1990, Gage, 1998, Richmond and Jorgensen, 1999).

\subsubsection{Endocytosis}

After vesicle fusion, the vesicle membrane and proteins have to be retrieved via endocytosis and recycled to ensure equilibrium of the vesicle pool and maintenance of the presynaptic surface area (Miller and Heuser, 1984). Several different modes of endocytosis have been proposed, the three major modes being kiss-and-run, bulk 
endocytosis and the most common and well-established clathrin-mediated endocytosis (Fig. II.4) (reviewed in Jung and Haucke, 2007, Rizzoli and Jahn, 2007).

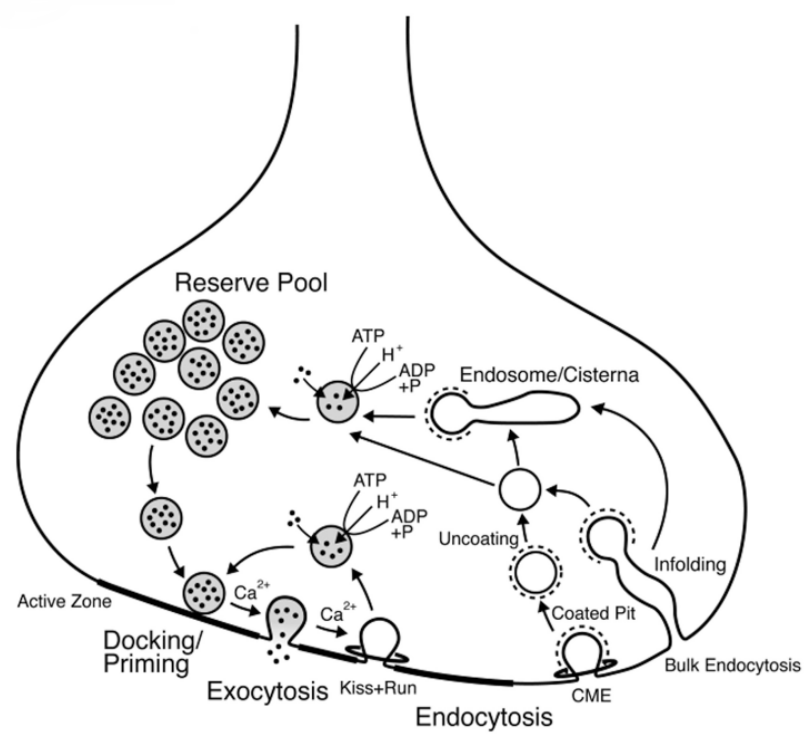

Fig. II.4 Modes of endocytosis. After exocytosis, vesicles can be retrieved via different modes of endocytosis: (1) "Kissand-run" follows a transient formation of a fusion pore. The original vesicle is pinched off right away. (2) Clathrin mediated endocytosis via formation of a clathrincoated pit. (3) Bulk endocytosis follows strong stimulation where many vesicles fuse and therefore cause a fast increase in membrane area. Large amounts of membrane invaginate into the presynaptic terminal and become endosome-like intermediates. Vesicles can be formed via clathrin-depended mechanisms from there. Reprinted from (Haucke and Klingauf, 2007) Endocytosis in neurons. In: A. Bean (Ed.) Protein Trafficking in Neurons, with permission from Elsevier.

Vesicle recycling via clathrin coating is thought to occur in four major steps: (1) Vesicle proteins and adaptor proteins such as AP180 and AP-2 are recruited to the membrane (Schmid, 1997, Haucke and De Camilli, 1999, Nonet, 1999). (2) Adaptor proteins recruit clathrin to form a clathrin-coated pit invaginating into the cytoplasm. The synaptic proteins endophilin and synaptojanin have been implicated in this budding process, possibly by acting on membrane composition and curvature (Harris et al., 2000, Huttner and Schmidt, 2000, Farsad et al., 2001, Schuske et al., 2003). (3) Fission of these coated pits from the plasma membrane is mediated by the GTPase dynamin, which forms a helical polymer around the membrane constriction. Upon GTP hydrolysis the coated vesicle is detached from the plasma membrane (Sweitzer and Hinshaw, 1998). (4) The uncoating from the clathrin cage was shown to be accomplished by the ATPase Hsc70, which is recruited via auxilin in vitro and in vivo (Ungewickell et al., 1995, Greener et al., 2001). 


\subsubsection{Vesicle recycling}

After endocytosis, vesicles may either directly rejoin the vesicle pool or, as several studies suggest, go through an endosomal sorting step (e.g. Wucherpfennig et al., 2003, Rizzoli et al., 2006) (Fig. II.4). To prepare the newly recycled SVs for exocytosis a vacuolar $\mathrm{H}+$ ATPase is responsible for re-acidification (Takamori et al., 2006). The established proton electrochemical gradient fuels the neurotransmitter uptake by neurotransmitter transporters (reviewed in Ahnert-Hilger et al., 2003).

\subsection{The active zone}

Triggered fusion of SVs with the presynaptic membrane occurs at the presynaptic $A Z$, a specialized area opposite to the postsynapse consisting of an elaborate protein network forming the cytomatrix at the active zone (CAZ). In electron micrographs this $A Z$ is characterized by an electron dense projection (DP), which is thought to be involved in the spatial organization of components such as calcium channels and the SV release and retrieval machinery (reviewed in Dresbach et al., 2001)

Five protein families are known to play an important role for the functionality of the $A Z$ (reviewed in Schoch and Gundelfinger, 2006). Protein structure with important domains and identified interactions are shown in Figure II.5 and II.6.

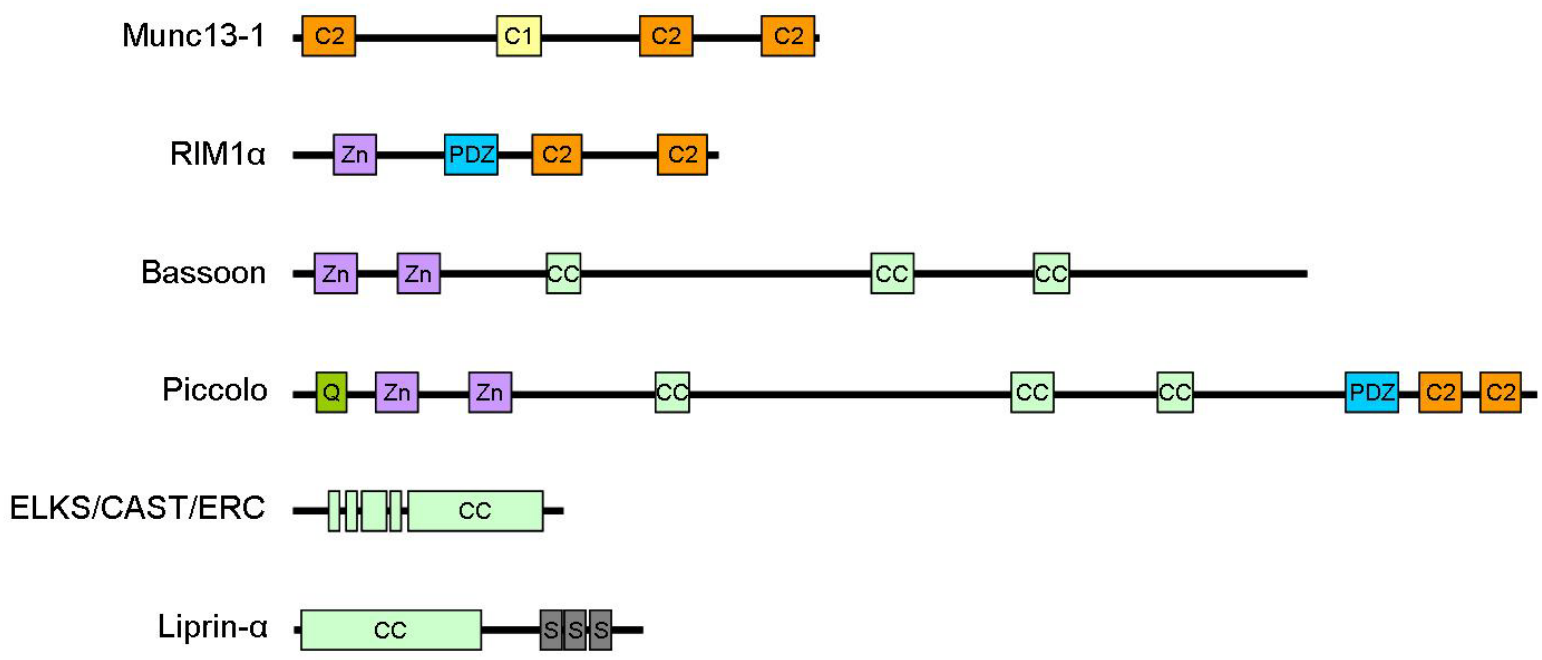

Fig. II.5 Protein structure and domains of CAZ proteins. Munc13-1 contains three C2-domains involved in calcium binding and a C1-domain involved in diacyl-glycerol and phorbolesters binding. RIM1a exhibits two C2-domains, an N-terminal zinc-finger domain ( $\mathrm{Zn}$ ) and a PDZ-domain involved in numerous protein interactions. Both Bassoon and Piccolo have two zinc-finger domains and three coiled-coil (CC) domains. Piccolo additionally contains a PDZ domain, two C2-domains and proline- 
rich sequences (Q). ELKS/CAST/ERC consists mainly of coiled-coil (CC) domains. Liprin- $\alpha$ is composed of $\mathrm{N}$-terminal coiled coil domains (CC) and $\mathrm{C}$-terminal sterile-alpha-domains (S) involved in recruiting other AZ proteins. Adapted from (Schoch and Gundelfinger, 2006).

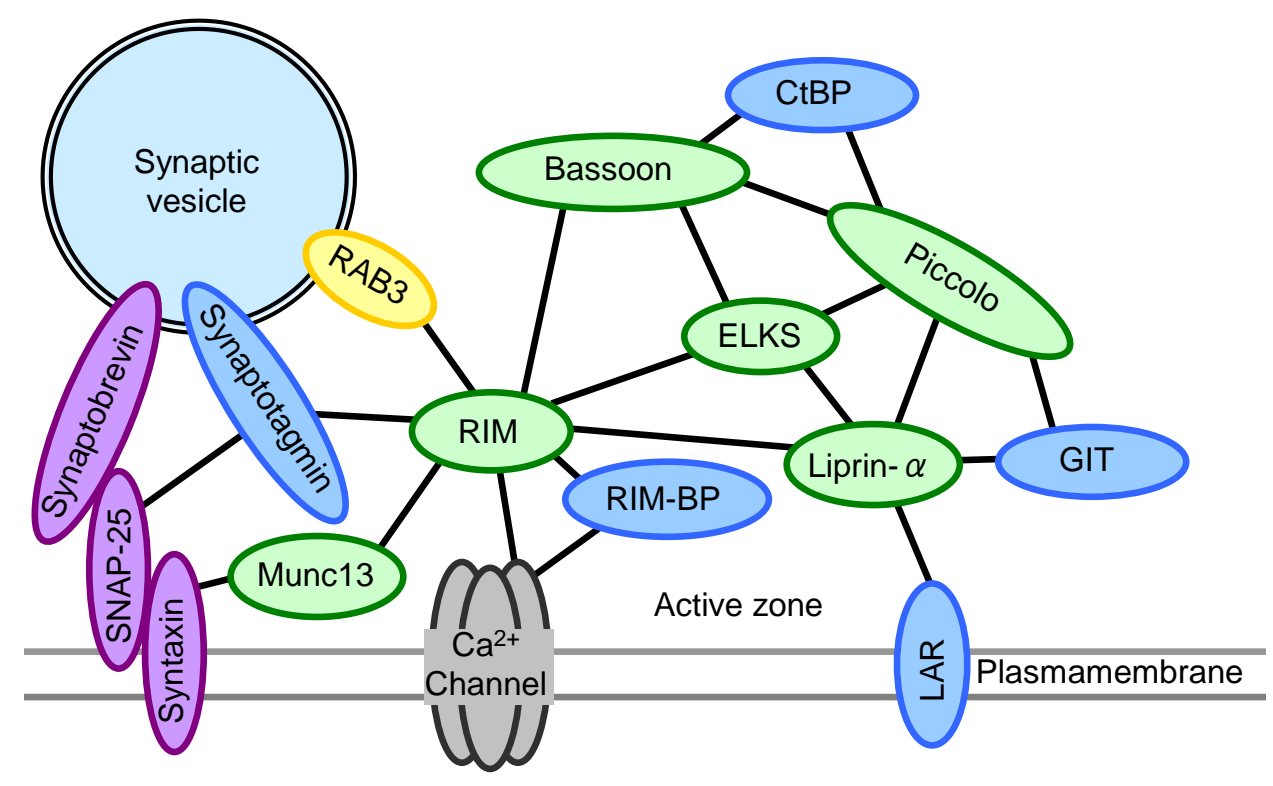

Fig. II.6 Protein interactions in the AZ. AZ enriched proteins are shown in green, SNARE proteins in purple, GTPases in yellow and other presynaptic proteins in blue or gray. (ELKS) ERC/RAB6interacting/CAST; (GIT) G-protein coupled receptor kinase-interactor; (LAR) LCA-related protein tyrosine phosphatase; (Rab) Ras-related in brain; (RIM) Rab interacting molecule; (RIM-BP) RIMbinding protein; (SNAP) synaptosome associated protein. Adapted from (Mittelstaedt et al., 2010).

\subsubsection{Munc13 protein family}

In vertebrates four Munc13 homologs (Munc13-1, Munc13-2, Munc13-3 and Munc134) are known. Except for the splice variant ubMunc13-2 (Koch et al., 2000), they are expressed exclusively in the brain, specifically at synapses (Brose et al., 1995). The C. elegans homolog uncoordinated (unc)-13 exhibits two splice isoforms (Kohn et al., 2000). All Munc13 isoforms share a $C 1$ domain that can bind the second messenger diacylglycerol and a central Munc homology domain flanked by two C2 domains (Fig. II.5) (Koch et al., 2000).

Several interactions of Munc13-1 with other CAZ proteins have been demonstrated: for example RIM1a (Betz et al., 2001) and syntaxin (Betz et al., 1997) (Fig. II.6). Currently, Munc-13/UNC-13 is believed to play a crucial role in priming of SVs via syntaxin interaction, thereby preparing them for release (Ashery et al., 2000, Weimer et al., 2006). 


\subsubsection{RIM proteins}

RIM was initially identified as Rab3a interacting molecule (Wang et al., 1997). Again, several homologs and isoforms are present in vertebrates whereas only one RIM homolog (UNC-10) has been identified in C. elegans (Koushika et al., 2001). RIM proteins are localized in the plasma membrane within $100 \mathrm{~nm}$ of the presynaptic DPs (Weimer et al., 2006). They belong to a family of scaffolding proteins that are composed of zinc-finger, PDZ and C2 domains (Fig. II.5) and interact with various proteins besides Rab3a: Munc13-1 (Betz et al., 2001); ELKS/CAST (Ohtsuka et al., 2002); synaptotagmin, SNAP-25 and voltage-dependent calcium channels (Coppola et al., 2001) and Liprin- $\alpha$ (Schoch et al., 2002) (Fig. II.6). In C. elegans unc-10 mutants a reduction of docked vesicles adjacent to the DP has been proposed and was later verified by EM tomography (Weimer et al., 2006, Stigloher et al., 2011). These finding suggesting that RIM holds SVs close to the DP and AZ membrane to facilitate docking and priming, possibly through simultaneous Rab3 and UNC-13 binding (Dulubova et al., 2005).

\subsubsection{ELKS/CAST/ERC proteins}

Members of this protein family consist mostly of coiled coil domains (Fig. II.5) and were identified in several independent screens, hence the various names. ELKS are glutamate $(E)$, leucine $(L)$, lysine $(K)$ and serine $(S)$ rich proteins and were first described in papillary thyroid carcinoma to activate a cytoplasmic tyrosine kinase (Nakata et al., 1999, Nakata et al., 2002). CAST (CAZ-associated structural protein) was isolated from rat brain membrane fractions and found to localize at synapses by immuno-histochemistry and immuno-EM (Ohtsuka et al., 2002). ERC (ELKS/Rab6interacting protein/CAST) was identified in a yeast-two-hybrid screen for proteins interacting with the PDZ domain of RIM (Wang et al., 2002).

The N-terminus of the Drosophila AZ protein Bruchpilot is partially homologous to human and C. elegans ELKS (Wagh et al., 2006) and was recently identified as major structural component of the Drosophila NMJ and photoreceptor dense projection (T-bar) (Kittel et al., 2006, Wagh et al., 2006, Fouquet et al., 2009). At synaptic sites, loss of BRP leads to impaired calcium channel clustering in the AZ, loss of T-bars and reduced evoked response (Kittel et al., 2006). Like all CAZ proteins, also ELKS interacts with other AZ proteins: RIM1, Munc13-1 (Ohtsuka et 
al., 2002) and Liprin-a (Ko et al., 2003b) (Fig. II.6). To date, only one ELKS homolog is known in C. elegans (Ohtsuka et al., 2002). Although no synaptic defects could be distinguished in $C$. elegans elks-1 loss-of-function mutants, ELKS-1 does play a crucial role in regulating functionality of SYD-2 (Dai et al., 2006), a scaffolding protein implicated in AZ assembly (see 2.2.5 and III).

\subsubsection{Piccolo and Bassoon}

Piccolo and Bassoon are structurally related proteins (Fenster et al., 2000) and the largest of the known CAZ proteins (Cases-Langhoff et al., 1996, tom Dieck et al., 1998). Through their zinc fingers and coiled coil domains (Fig. II.5), binding to other proteins like ELKS (Takao-Rikitsu et al., 2004) and dual prenylated Rab3A and synaptobrevin2/NAMP2 (Fenster et al., 2000) is mediated. Piccolo additionally has a PDZ domain interacting with CAMPGEFII (Fujimoto et al., 2002). The C2 domains bind to L-type voltage-dependent calcium channels (Shibasaki et al., 2004) and forms calcium-dependent homo- and heterodimers with Rim2 (Fujimoto et al., 2002) suggesting a regulatory function in synaptic transmission as calcium sensor (Fig. II.6).

Mouse Bassoon mutants encounter free floating ribbons, disturbed localization of $A Z$ components and impaired synaptic transmission in retina and cochlear hair cells (Dick et al., 2003, Khimich et al., 2005, Frank et al., 2010), suggesting a function in synapse assembly in vertebrates. To date, no homologs were identified in C. elegans and Drosophila.

\subsubsection{Liprin- $\alpha$ proteins}

Liprin- $\alpha$ proteins are scaffolding proteins with $\mathrm{N}$-terminal coiled coil domains and three C-terminal sterile alpha motif (SAM) domains (Fig. II.5) originally identified as LAR Interaction Protein (LIP.1). Vertebrate Liprin- $\alpha 2$ and - $\alpha 3$ are brain-specific, while Liprin- $\alpha 1$ and - $\alpha 4$ are expressed also outside the nervous system (Serra-Pages et al., 1998). With their C-terminal coiled coil domains, they interact with RIM (Schoch et al., 2002), ELKS (Ko et al., 2003b) and G protein-coupled receptor kinase-interacting protein (GIT) (Ko et al., 2003a) (Fig. II.6). They additionally bind to the SV motor 
protein KIF1A/UNC-104 (Shin et al., 2003) and the postsynaptic glutamate receptor interacting protein (GRIP) (Wyszynski et al., 2002).

The invertebrates Drosophila and C. elegans carry only one liprin-a gene: Dliprin- $\alpha$ and syd-2, respectively. Dliprin-a was shown to be required for normal presynaptic AZ morphology and proper synaptic transmission (Kaufmann et al., 2002). The $C$. elegans homolog SYD-2 (synapse defective 2) was isolated in a genetic screen for mutations affecting SV localization in C. elegans motor neurons (Zhen and Jin, 1999). SYD-2 has been implicated in the recruitment of several synaptic proteins to presynaptic DPs suggesting it to be a key regulator of DP assembly (Zhen and Jin, 1999; Patel et al., 2006).

Although many AZ proteins have been identified and various genetic and biochemical interactions were demonstrated, the mechanism by which these proteins form the highly complex CAZ is largely unknown. In fact, most proteins localize independently of each other to synaptic sites (Koushika et al., 2001, Deken et al., 2005).

\subsection{DP architecture in different organisms}

As described above, the $A Z$ is the most crucial structure of the synapse to regulate and control synaptic transmission. Due to its proteinaceous content it can easily be identified in the electron microscope as dense structure. Typically a cloud of SVs clusters around it. In this study I focused on the 3D ultrastructure of the electron dense projection (DP) at the neuromuscular junctions (NMJ) in C. elegans and its function in synaptic transmission. Although a DP is found in almost every synapse and the same function of tethering SVs to the $A Z$ has been proposed, the architecture differs severely between different types of synapses and in different organisms (reviewed in Zhai and Bellen, 2004).

NMJ DPs in C. elegans (Fig. II.7 A) were described as rather simple and plaque-like (White et al., 1986), while Drosophila exhibits a more complex T-bar shaped DP (Prokop and Meinertzhagen, 2006) (Fig. II.7 B). Frog NMJs were described by Harlow and colleagues as array-like structure with beams and ribs connecting docked vesicles to putative calcium channels (Fig. II.7 C) (Harlow et al., 2001). 


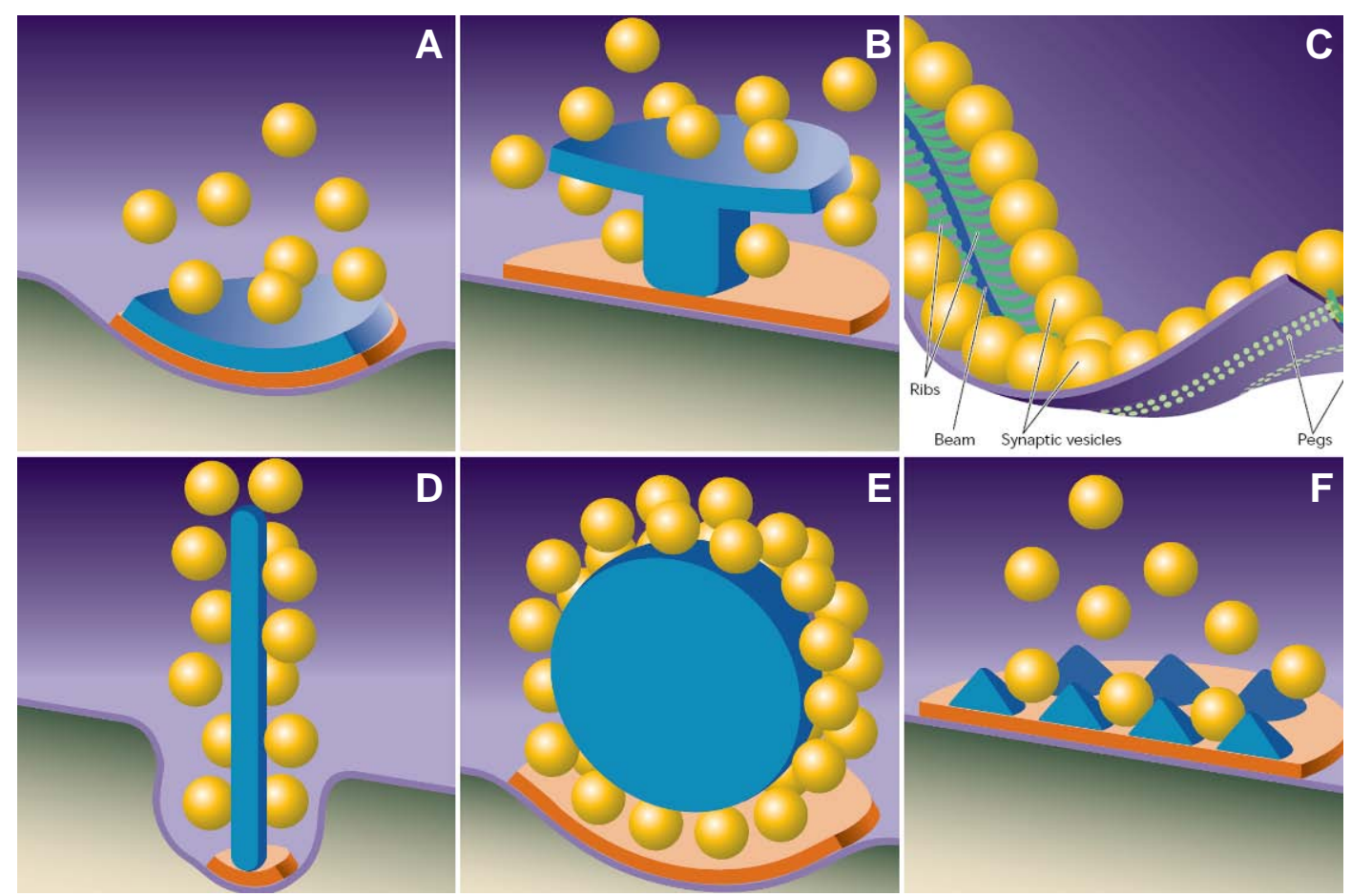

Fig. II.7 DP architecture in different organisms and synapse types. Neuromuscular junction DPs (blue) are highly divers between C. elegans (A), Drosophila (B) and frog (C). DPs of sensory neurons employ large DP structures to ensure rapid SV (yellow) replenishment during continuous release. Examples are ribbon synapses in photoreceptors of the retina (D) and ribbons in frog saccular hair cells (E). AZs in the mammalian central nervous system contain a web-like organization of small DPs with interspaces for SV docking and fusion (F). Taken and modified from (Zhai and Bellen, 2004).

Sensory synapses are usually characterized by a very large DP that can tether a multitude of SVs. This feature likely reflects their function in replenishing the readily releasable SV pool that is needed for the continuous release in sensory neurons (Lenzi and von Gersdorff, 2001). Examples are the ribbon synapses in photoreceptors and bipolar cells in the retina (Fig. II.7 D) and in frog saccular hair cells (Fig. II.7 E). Mammalian central nervous system synapses on the other hand exhibit a web-like pattern of small DPs forming 50-100 nm slots for SV docking and fusion (Fig. II.7 F) (Phillips et al., 2001).

Although many $A Z$ proteins have been identified (see also 2.2), the precise molecular composition of the AZ DP is largely unknown as are the mechanisms for their assembly. It has been proposed that $A Z$ proteins are transported via dense core vesicles (DCVs) to nascent synapses (Zhai et al., 2001, Shapira et al., 2003). These transport vesicles of $80 \mathrm{~nm}$ size were isolated from rat brain lysates with Piccolo antibodies and co-purified with other AZ proteins as syntaxin, SNAP25 and Bassoon (Zhai et al., 2001) as well as Munc18, Munc13, Rab3a/c, RIM and subunits of the N- 
type calcium channel (Shapira et al., 2003). DCVs of similar size and morphology were observed in cultured hippocampal neurons and were positively labeled for Piccolo and Bassoon (Zhai et al., 2001), supporting the hypothesis of DCVassociated transport of $A Z$ components to presynaptic sites.

Several lines of evidence suggest that components vary between different synapse types and organisms. For retina ribbon synapses, RIBEYE has been shown to be the major constituent (Schmitz et al., 2000), whereas Drosophila T-bar formation strongly depends on Bruchpilot as structural component (Kittel et al., 2006, Wagh et al., 2006, Fouquet et al., 2009). For $C$. elegans, many proteins involved in the $A Z$ assembly have been identified, yet the structural components of the DP are unknown.

\subsection{C. elegans as a model system}

The nematode Caenorhabditis elegans was introduced as a model in the 1960s by Sydney Brenner to study neurobiology in a simple multi-cellular organism. The adult worm is only about $1 \mathrm{~mm}$ long and $65 \mu \mathrm{m}$ in diameter and its anatomy is very simple. Animals are fed with Escherichia coli (OP50) on agar plates and have a very fast life cycle (3 days). The transparency of the worm is ideal for live fluorescence and bright field imaging. C. elegans was the first metazoan whose genome was completely sequenced (C. elegans Sequencing Consortium, 1998) and a tremendous amount of genetic tools is available. Most worms are hermaphrodites and only a small fraction are males. Hermaphrodites usually reproduce via self-fertilization but progeny can also be generated by crossing with males, which offers the possibility of genetic studies. Mutants are often viable and homozygotes can easily be maintained. The formation of dauer larva allows storage of mutant strains over month. These and other numerous advantages have lead to a wide use of $C$. elegans as a model organism (reviewed in Riddle et al., 1997).

One of the greatest advantages for studies in C. elegans is the constancy of somatic cell number (eutely) and stereotypic cell positions and connections between individual animals. This includes the nervous system, which is composed of exactly 302 neurons in hermaphrodites. The nervous system consists of the nerve ring, head and tail ganglia and a dorsal and a ventral nerve cord (Fig. II.8). C. elegans neurons have a very simple morphology, usually with an unbranched axon and dendrite 
following a trajectory highly stereotypic between individuals. A complete neural connectivity map is available for in C. elegans due to serial EM reconstruction by John White (White et al., 1986).

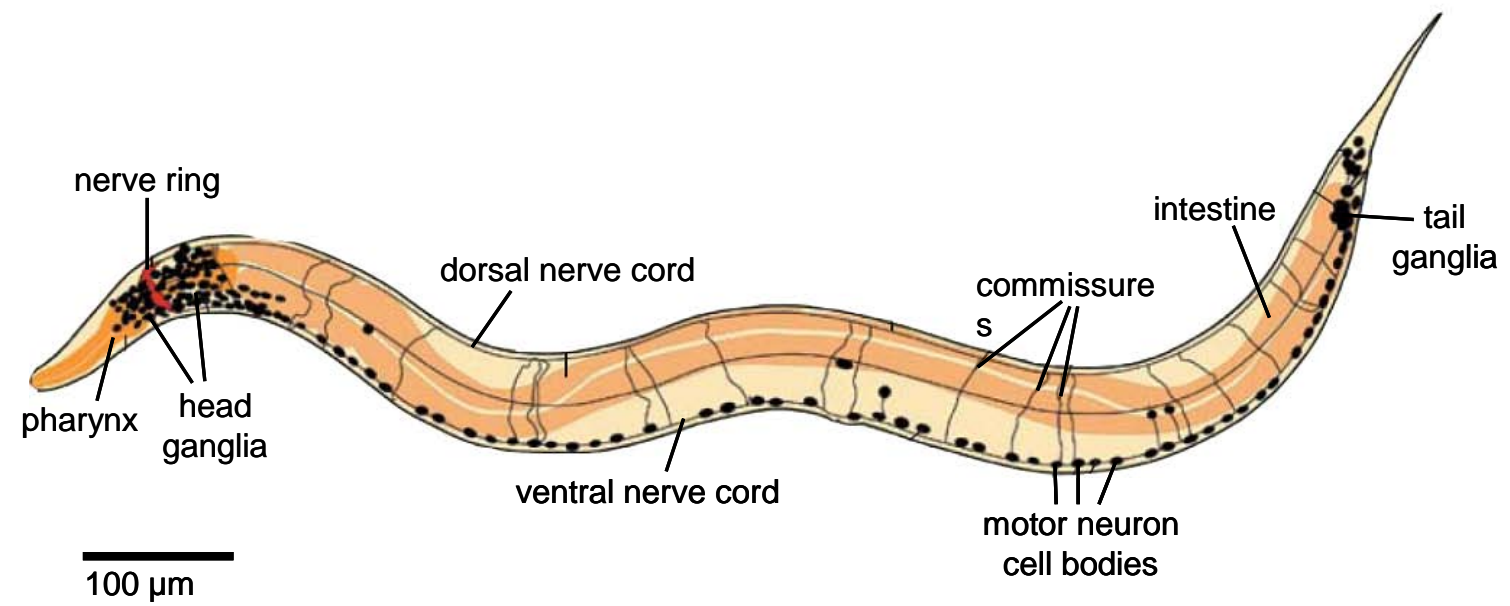

Fig. II.8 The nervous system of C. elegans. The head is oriented to the left with the nerve ring and head ganglia containing most of the neuronal cell bodies. A dorsal and a ventral nerve cord extend from the nerve ring to the tail ganglia. Cell bodies of cholinergic and GABAergic motor neurons innervating the body wall muscles are localized in the ventral nerve cord. Axons and dendrites reach the dorsal side via commissures. Image taken and modified from (Gally and Bessereau, 2003).

\subsection{The neuromuscular system of $C$. elegans}

The neuromuscular system of $C$. elegans is composed of body wall muscles and motor neurons that run in two nerve cords (ventral and dorsal) along the anteriorposterior axis of the animal. The cell bodies of the motor neurons innervating the dorsal and ventral body wall muscles (BWM) are localized exclusively in the ventral nerve cord. To innervate dorsal body wall muscles or receive synaptic input at NMJs, neurons must extend axons or dendrites via commissures to the dorsal side (Fig. II.8) using the UNC-6/netrin and SLT-1/slit pathways (reviewed in Chisholm and Jin, 2005). NMJs are formed en passant in local swellings of the axons and aligned like "pearls on a string" along the ventral and dorsal nerve cords (White et al., 1986). This allows easy imaging with light and fluorescence microscopy as well as fast identification of NMJs in electron micrographs. The number of synapses formed is relatively invariant between animals (White et al., 1986), which allows direct quantification of synaptic assembly defects via observation of synapse number, size and morphology. The possibility to fuse fluorescent proteins like GFP to proteins and 
express them in compartments or tissues of interest (Chalfie et al., 1994, Tsien, 1998) allows easy screening for defects in synapse assembly or vesicle mislocalization in C. elegans (Nonet, 1999).

The 95 longitudinal body wall muscles responsible for $C$. elegans locomotion are organized in four quadrants on both sides of the nerve cords (Fig. II.9) (Sulston and Horvitz, 1977). Opposed to most other nervous systems, nematode muscle cells extend muscle arms towards the nearest nerve cord to form NMJs rather than neurons sending axons towards the muscle cells (Stretton, 1976) (Fig. II.9).
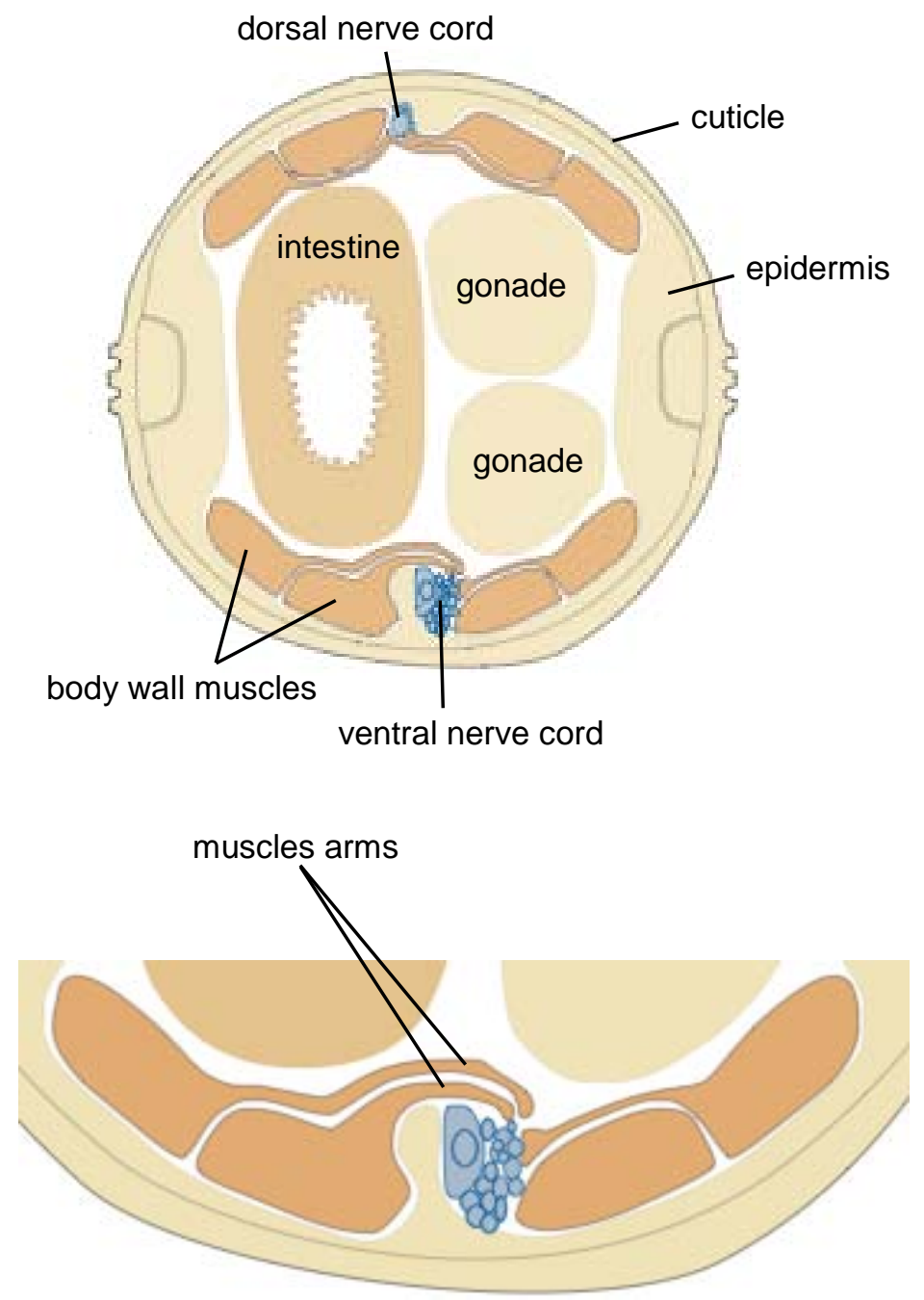

Fig. II.9 Muscle arms extend from body wall muscles to form neuromuscular junctions. The scheme shows a $C$. elegans cross section. Two rows of body wall muscles on each side of the nerve cords form a quadrant. They extend muscle arms towards the adjacent cord to form neuromuscular junctions. Image taken and modified from (Gally and Bessereau, 2003).

Forward and backward locomotion on solid surfaces is accomplished by sinusoidal body bends that pass wave-like through the length of the body. The body bends are 
generated by simultaneous contraction of body wall muscles (BWM) on one side and relaxation on the opposite side. Two types of motor neurons realize the reciprocal activation and inhibition: Cholinergic motor neurons releasing Acetylcholine (ACh) and GABAergic motor neurons releasing $\gamma$-Aminobutyric acid (GABA), respectively (McIntire et al., 1993, Richmond and Jorgensen, 1999) (Fig. II.10 and II.11).

presynaptic terminal

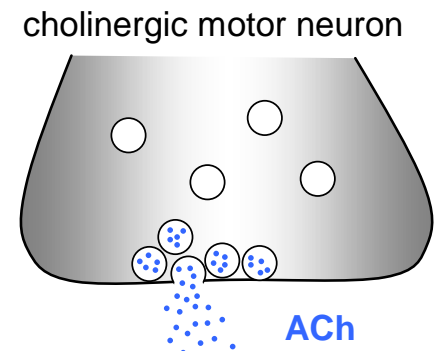

GABAergic motor neuron

postsynaptic membrane
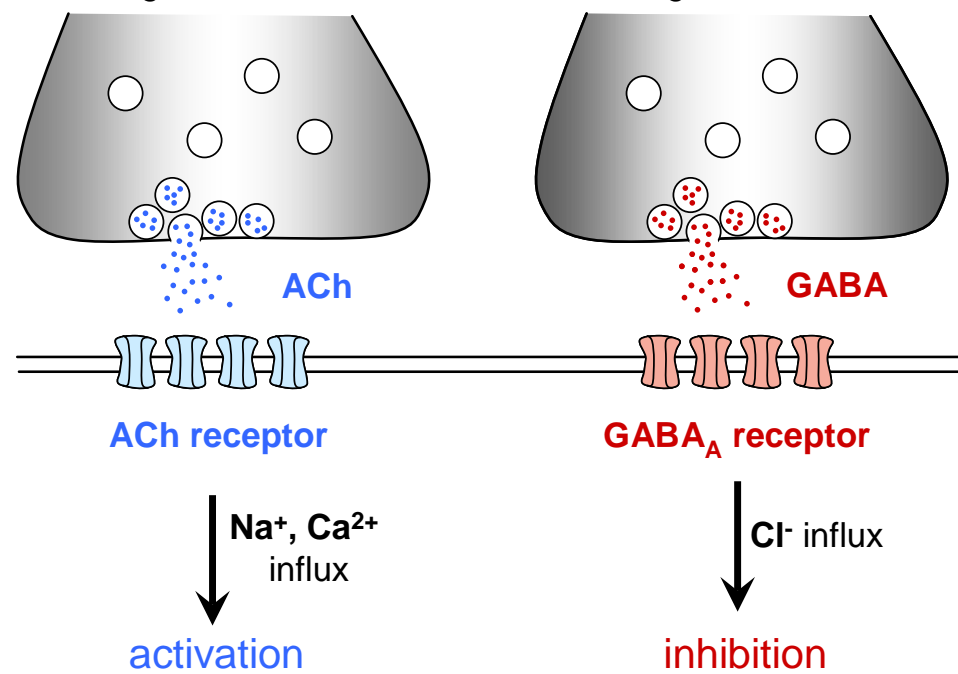

activation

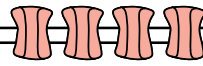

$\mathrm{GABA}_{\mathrm{A}}$ receptor

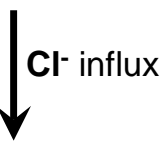

inhibition

Fig. II.10 Types of motor neurons in C. elegans. Acetylcholine (ACh) released from cholinergic neurons binds to postsynaptic nicotinic ACh receptors and causes muscle activation/contraction through $\mathrm{Na}+$ and $\mathrm{Ca} 2+$ influx. On the contrary, $\gamma$-Aminobutyric acid (GABA) released from GABAergic neurons binds to GABAA receptors and causes muscle inhibition/relaxation by opening channels permeable for cloride ions.

Postsynaptic acetylcholine receptors are non-selective cation channels. Binding of ACh is followed by an influx of sodium and calcium ions and leads to the depolarization of the muscle cell. The BWM contracts upon this activation. In contrast, $\mathrm{GABA}_{A}$ receptors are ion channels permeable for chloride. Binding of GABA causes an influx of chloride and consequently leads to hyperpolarization and relaxation of the muscle (Fig. II.10 and II.11) (Richmond and Jorgensen, 1999). Two distinct sets of motor neurons are responsible for forward and backward movement: Excitatory cholinergic B-type motor neurons (DB and VB) are involved in forward movement, while cholinergic A-type motor neurons (DA and VA) render backward movement. The cross-inhibition is in both cases carried out by the inhibitory GABAergic DD and VD neurons (reviewed in de Bono and Maricq, 2005). 


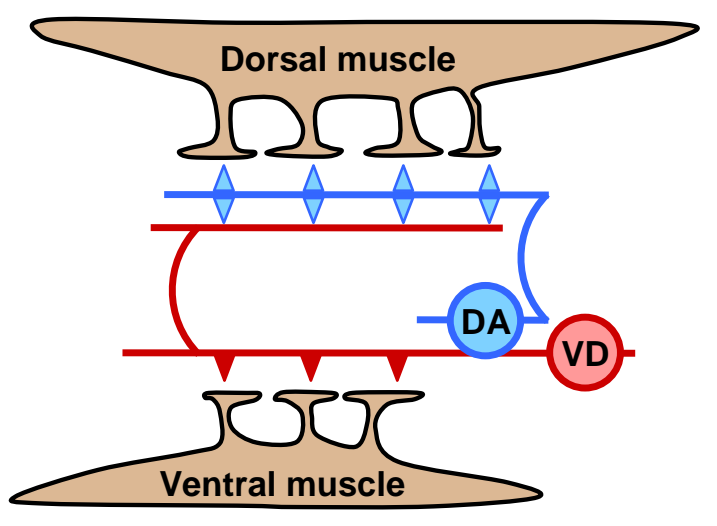

\section{$\Delta$ Acetylcholine, excitatory}

$\boldsymbol{\nabla}$ GABA, inhibitory
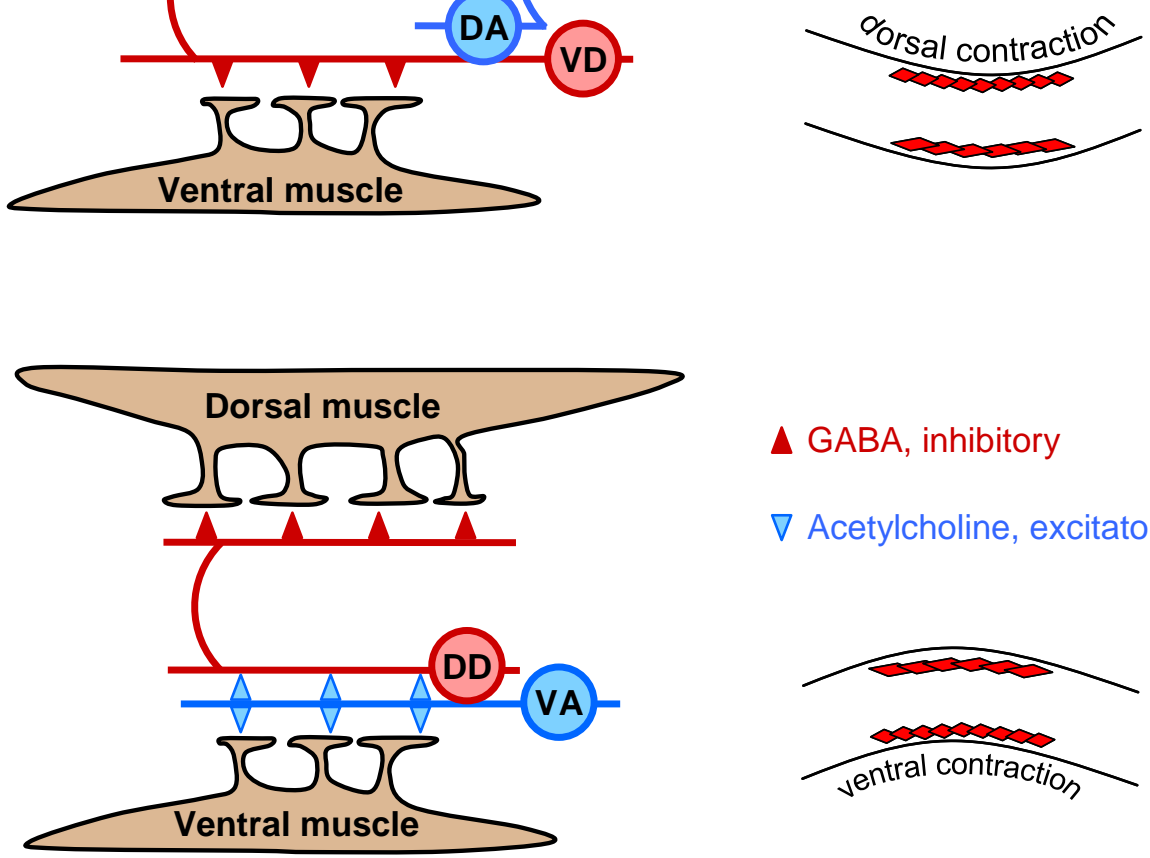

$\triangle$ GABA, inhibitory

$\nabla$ Acetylcholine, excitatory

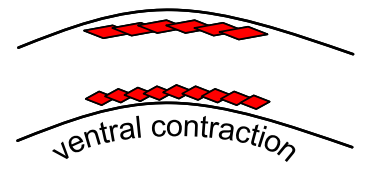

Fig. II.11 Alternating contraction of dorsal and ventral body wall muscles. Top: Excitatory cholinergic DA neurons extend their axons via commissures to the dorsal cord to form dyadic synapses, which release ACh and activate the dorsal BWMs, leading to contraction. The same synapses simultaneously innervate inhibitory VD dendrites. The stimulus is transmitted to the axons of the VDs in the ventral cord where GABA release is triggered and leads to inhibition and relaxation of the opposite ventral BWMs. Bottom: Similarly, ventral BWMs are excited via VA neurons leading to contraction. VA neurons simultaneously innervate inhibitory DD dendrites leading to release of GABA at their dorsal synapses, which results in relaxation of dorsal BWMs.

In electron micrographs, GABAergic and cholinergic motor neurons can be discriminated by their postsynaptic partners. While GABAergic synapses exclusively project onto body wall muscles and muscle arms (Schuske et al., 2004), cholinergic synapses project onto both, muscles and the dendrites of GABAergic neurons (Mclntire et al., 1993) (Fig. II.12). 
GABAergic synapses
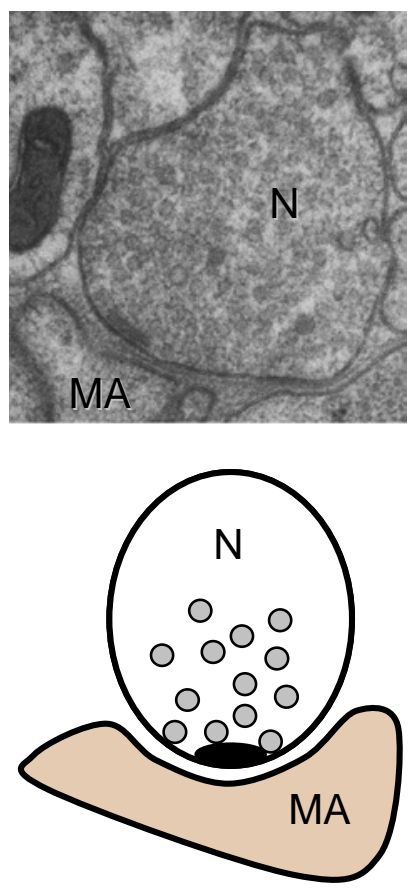
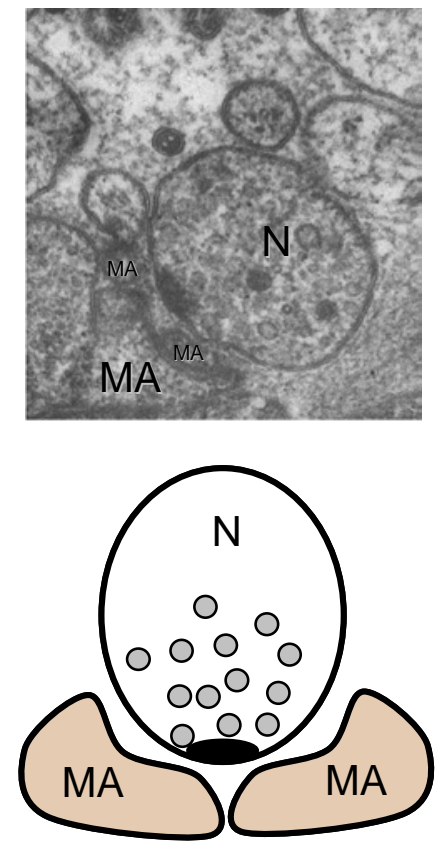

cholinergic synapses
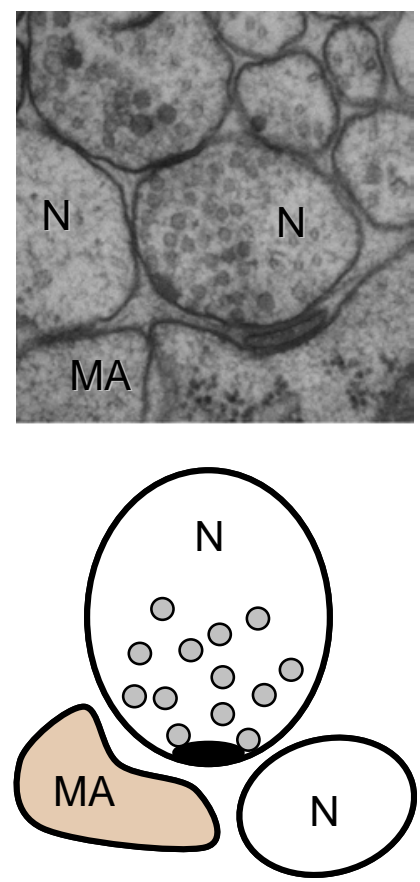

Fig. II.12 Differentiation of GABAergic and cholinergic synapses in C. elegans. Types of motor neurons $(\mathrm{N})$ were discriminated via their postsynaptic targets. GABAergic neurons exclusively project onto muscle cells and muscle arms (MA), while cholinergic neurons innervate muscle cells as well as dendrites of GABAergic neurons $(\mathrm{N})$.

Forward or backward locomotion is generated as a reaction to environmental cues of mechanical, thermal or chemical kind (Hedgecock and Russell, 1975, Chalfie et al., 1985, Pierce-Shimomura et al., 1999). A complex network of neuron-neuron interactions including a specific set of command interneurons has been identified to drive forward or backward locomotion upon mechanosensation (reviewed in de Bono and Maricq, 2005, Goodman, 2006). Anterior body touch is recognized by the sensory neurons AVM (anterior ventral microtubule (MT) cell) and ALM (anterior lateral MT cell), whereas posterior body touch is detected by sensory neurons PVM (posterior ventral MT cell) and PLM (posterior lateral MT cell). These sensory neurons transmit the electrical stimulus to command interneurons via gap junctions and chemical synapses. In turn, the command interneurons activate motor neurons specific for the desired locomotion direction. A simplified wiring diagram is shown for backward movement upon anterior and posterior body touch (Fig. Il.13) (reviewed in de Bono and Maricq, 2005). 


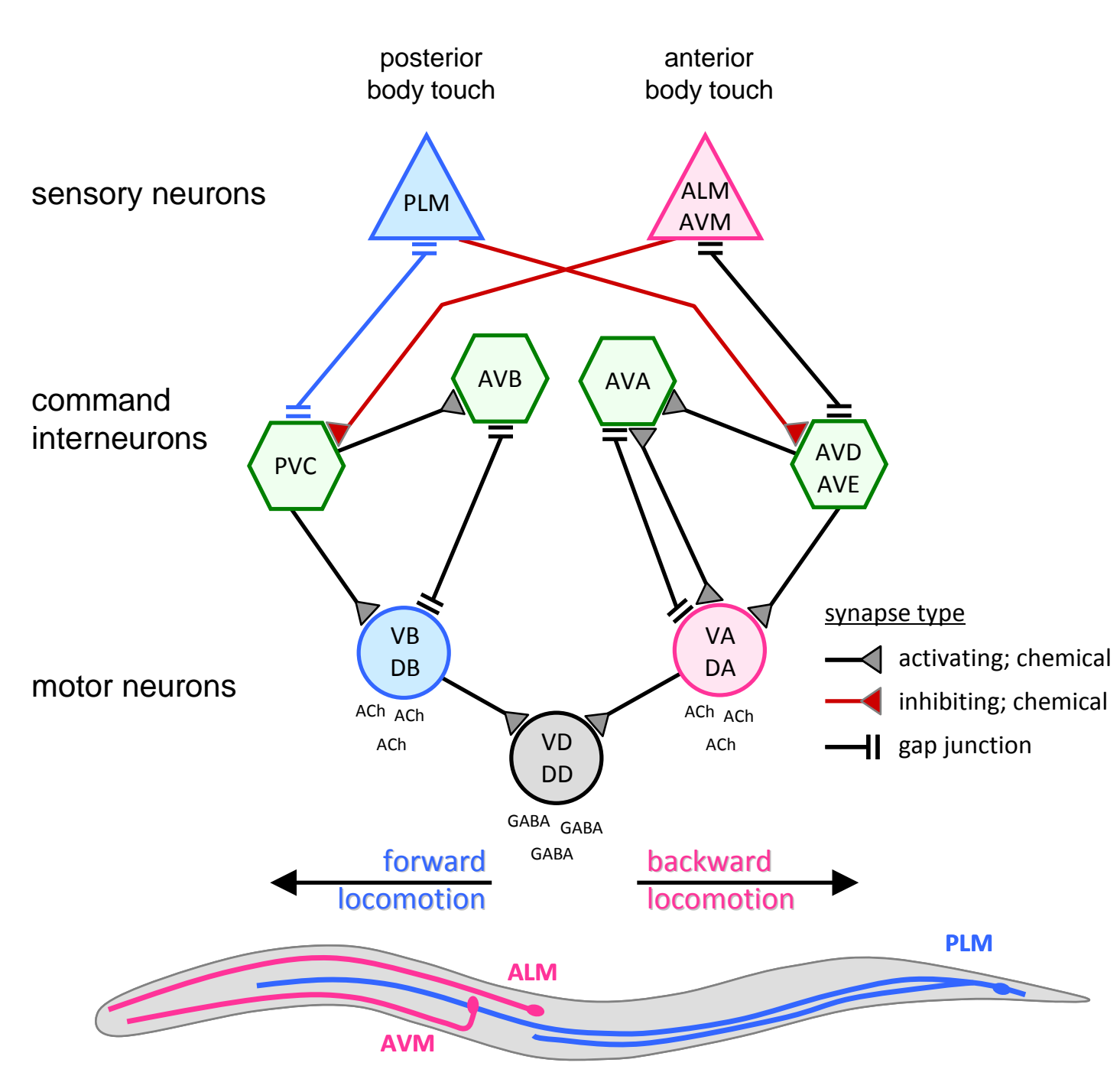

Fig. II.13 Neuronal wiring to elicit backward locomotion upon anterior and posterior body touch. Mechanosensory input resulting from body touch is transmitted via gap junctions from ALM/AVM or PLM touch neurons to the respective command interneurons AVD/AVE or PVC, respectively. Upon anterior touch, AVD and a second command interneuron AVA innervate the A-type cholinergic motor neurons (VA, DA) responsible for backward movement. Likewise, PVC and a second command interneuron (AVB) activate the B-type cholinergic VB and DB motor neurons responsible for forward movement upon posterior touch. Cholinergic motor neurons innervate muscles and simultaneously GABAergic DD and VD neurons to generate body bends. The circuit for forward locomotion is inhibited through touch neurons and interneurons controlling backward locomotion and vice versa. Adapted from (de Bono and Maricq, 2005) and (Altun and Hall, 2011). Not all synaptic connections are indicated.

As movement patterns of $C$. elegans are rather simple and controlled by a distinct set of neurons, analysis of locomotion can yield as an easy read out for impaired synaptic transmission in motor neurons. A specialized motor activity in C. elegans is 
egg-laying. Cholinergic VC4 and VC5 neurons as well as hermaphrodite specific neurons (HSN) are involved in innervating the vulva muscles to extrude eggs through the vulva opening (White et al., 1986, Shen et al., 2004). Similar to locomotion analysis, egg-laying behavior can be used as read out for impaired synaptic transmission.

\subsection{High-pressure-freezing and freeze substitution for electron microscopy}

Synapses in general are very small structures. Motor neuron synapses in C. elegans specifically are only about $500 \mathrm{~nm}$ in diameter. Our structures of interest, the AZ DPs and the surrounding SVs are even smaller with sizes of $200 \mathrm{~nm}$ and $30 \mathrm{~nm}$, respectively.

Despite recent advances in light microscopy (STED, STORM, PALM or TIRF) enhancing resolution to 15-20 $\mathrm{nm}$ for mapping proteins in cells (Donnert et al., 2006) and even $5.8 \mathrm{~nm}$ for imaging dense color centers in crystals (Rittweger et al., 2009), it is not sufficient to thoroughly analyze SV distribution and $A Z$ architecture. Therefore, this study is mainly based on EM analysis. Electron microscopes use a beam of electrons with a 100,000 times shorter wavelength than photons and therefore higher resolution to illuminate the specimen. Hence the resolution is much higher. Depending on the sample and the acceleration voltage, resolution can go down to the atomic level (Erni et al., 2009). Additionally, all structures in the tissue are displayed without specific labeling as is required for fluorescence microscopy. Some electrons can pass the sample unscattered and can be detected by a CCD (charge-coupled device) camera. Those electrons scattered by the atomic nucleus or the electron shell of a molecule in the sample are not detected. They are displayed as dark spots and therefore accounting for the contrast. Biological samples have an intrinsic low contrast due to the low atomic numbers of predominant carbon and nitrogen and hydrogen compounds. This contrast can be enhanced by additional staining of proteins and nucleotides with heavy metals like lead citrate and uranyl acetate (refer to 9.5.). One of the disadvantages of EM is, that samples have to be water-free, eliminating the possibility of imaging live tissue. They also need to be very thin (usually up to $200 \mathrm{~nm}$ ) for the electron beam to penetrate the tissue. This implies the need for special sample preparation. 
Conventional chemical fixation and dehydration is usually done at room temperature. The fixation depends on the diffusion of fixative with specific cross linkers into the tissue. Diffusion is limited by the diffusion rate and also by natural diffusion barriers like exoskeletons. While infiltration and cross-linking take place, cellular components can be degraded or translocated, limiting the quality of the ultrastructure and nativity of the tissue. In C. elegans, diffusion of fixative into the animal is strongly impaired due to the thick cuticle and worms are moving for hours in the fixing solution (Weimer, 2006). Disruption of the cuticle beforehand improves infiltration but at the same time disrupts the integrity of nearby tissue. Conventional preparation methods would therefore not allow us to reliably characterize the architecture of the $A Z$ in $C$. elegans.

To prevent alteration of cellular component localization, methods like plunge freezing (Sosa et al., 1994), jet spray and cold block (slamming) cryo fixation (Dubochet et al., 1988, Lupetti et al., 2005), were developed. Cryo-immobilized components cannot move during infiltration and dehydration and therefore nativity of the sample is well preserved. However, only samples of a few micrometers are applicable for these methods. The reason is the poor heat conductance of water, which leads to ice crystal formation within the tissue, strongly disrupting the cellular integrity.

The development of high-pressure-freezing (HPF) by Hans Moor provided a great tool to partially overcome these issues (Moor, 1987). It is based on the physical phenomenon that the freezing point of water is lowered under high pressure (2000 bar applied for HPF) and water becomes amorphous ice (also called vitreous ice), slowing down ice crystal nucleation and growth (Moor, 1987). Samples up to $6 \mathrm{~mm}$ in diameter and $600 \mu \mathrm{m}$ in thickness can be frozen (in liquid nitrogen at 2000 bar) by the available HPF machines (Leica/Baltech/Wohlwend). Due to its small size, $C$. elegans is especially well suited for HPF. The preservation of the ultrastructure and nativity of $C$. elegans can be strongly improved using HPF and FS compared to classic chemical fixation (Rostaing et al., 2004) (Fig. II.14). 


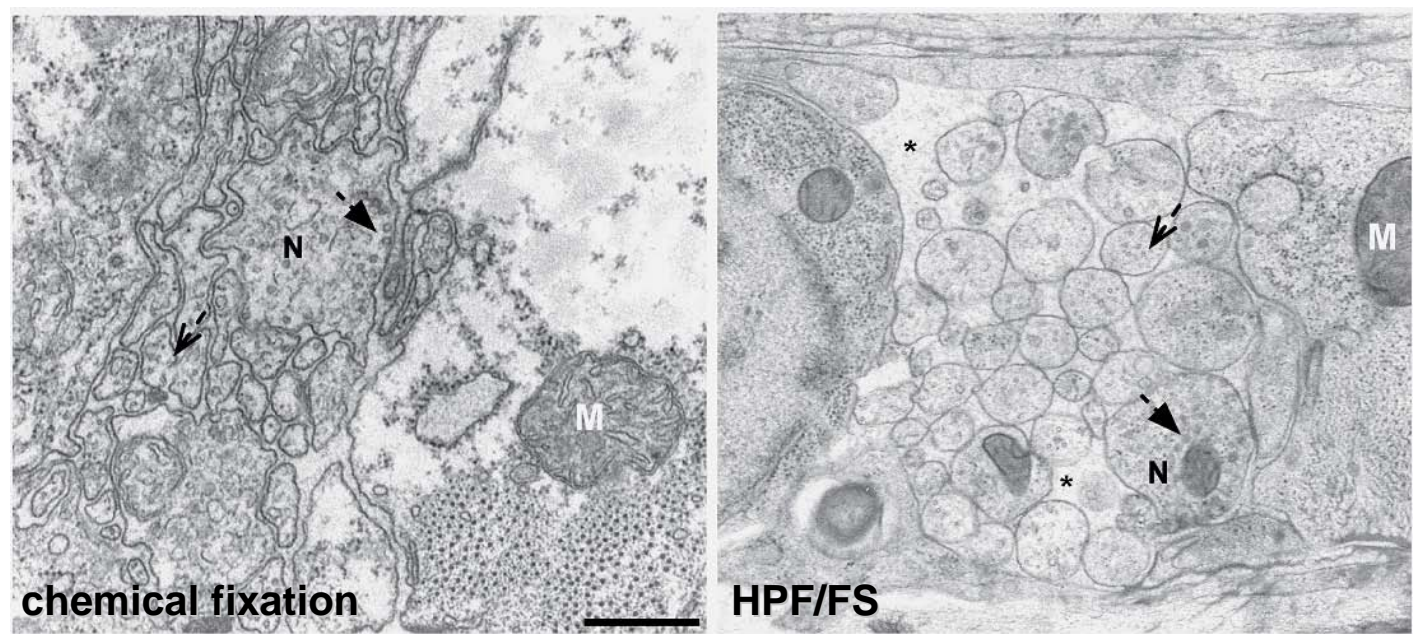

Fig. II.14 Cryofixation enhances ultrastructural preservation in C. elegans. Classical chemical fixation leads to artifacts like rippled membranes of neurons $(N)$ and compressed neuron processes within the nerve cord. This is caused by slow fixation and dehydration at room temperature. After HPF followed by FS, neurons are circular with interspaces. Arrows point out SVs (dashed arrow) and microtubules (open dashed arrow). Scale bar is $500 \mathrm{~nm}$. Reprinted from (Rostaing et al., 2004); with kind permission from Springer Science and Business Media.

20 to $30 \mathrm{C}$. elegans can easily be frozen in one specimen holder. While still cryoimmobilized at low temperatures $\left(-90^{\circ} \mathrm{C}\right)$, animals are slowly infiltrated with a fixative such as osmium tetroxide and tannin to cross-link lipids and macromolecules (refere to 9.3). Other fixatives like uranyl acetate or glutaraldehyde are also often used. During infiltration at low temperatures, water is slowly substituted by organic solvents. This method is called freeze substitution (FS). Finally, specimens are infiltrated and embedded at room temperature with hard epoxy resins for a good morphologic preservation or methacrylate-based resins for immune-labeling studies (Rostaing et al., 2004). Resins are hardened by heat or UV light. The plastic-embedded animal can then be cut with a diamond knife into thin sections needed for transmission EM (Fig. Il.15). 

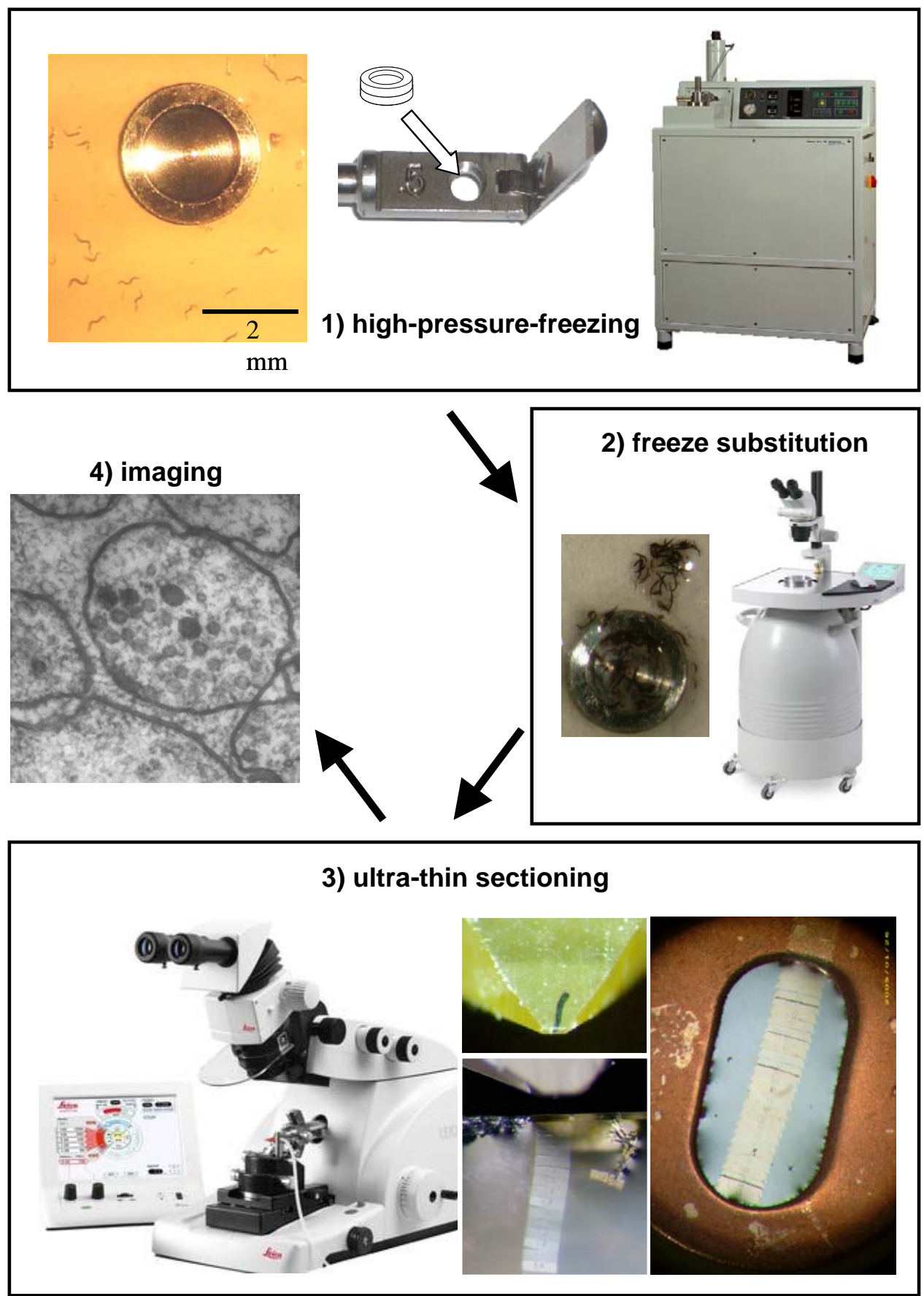

Fig. Il.15 Workflow from high-pressure-freezing to imaging. About 20 to 30 adult $C$. elegans were placed into freezing chambers (top left). Chambers were closed and transferred to a metal holder for HPF and rapidly frozen in a Baltech010 (top right). Frozen specimens were transferred in the chambers into a Leica automatic freeze substitution (AFS) machine at $-90{ }^{\circ} \mathrm{C}$ (right). Infiltration and fixation was done while temperatures were raised from $-90{ }^{\circ} \mathrm{C}$ to $-4{ }^{\circ} \mathrm{C}$ over days. Freeze-substituted worms are stained dark from osmium tetroxide (right). After embedding in plastic, worms were cut into thin serial sections on a Leica ultramicrotome and collected on formvar coated grids (bottom). Poststained sections were imaged at a Zeiss EM 902A at $80 \mathrm{kV}$ (left). Images are taken from http://www.spectraservices.com/HPM010.html; http://www.leica-microsystems.com/products/electronmicroscope-sample-preparation/biological-specimens/; photographs were kindly provided by Dr. Jan Hegermann. 
To precisely investigate the fine structure and relative localization of intracellular components to each other, we need 3D information. EM tomography of HPF and FS treated samples allows us to obtain complex 3D ultrastructural details of organelles and structures with a resolution down to about $5 \mathrm{~nm}$ (Frank et al., 2002). To resolve the architecture of the NMJ DP in C. elegans, thick sections of the nerve cords were imaged in the electron microscope from a number of angles (Fig. II.16). Backprojections from the obtained images can then be processed by a computer software and result in a 3D volume reconstruction of the sample (refer to 9.7).
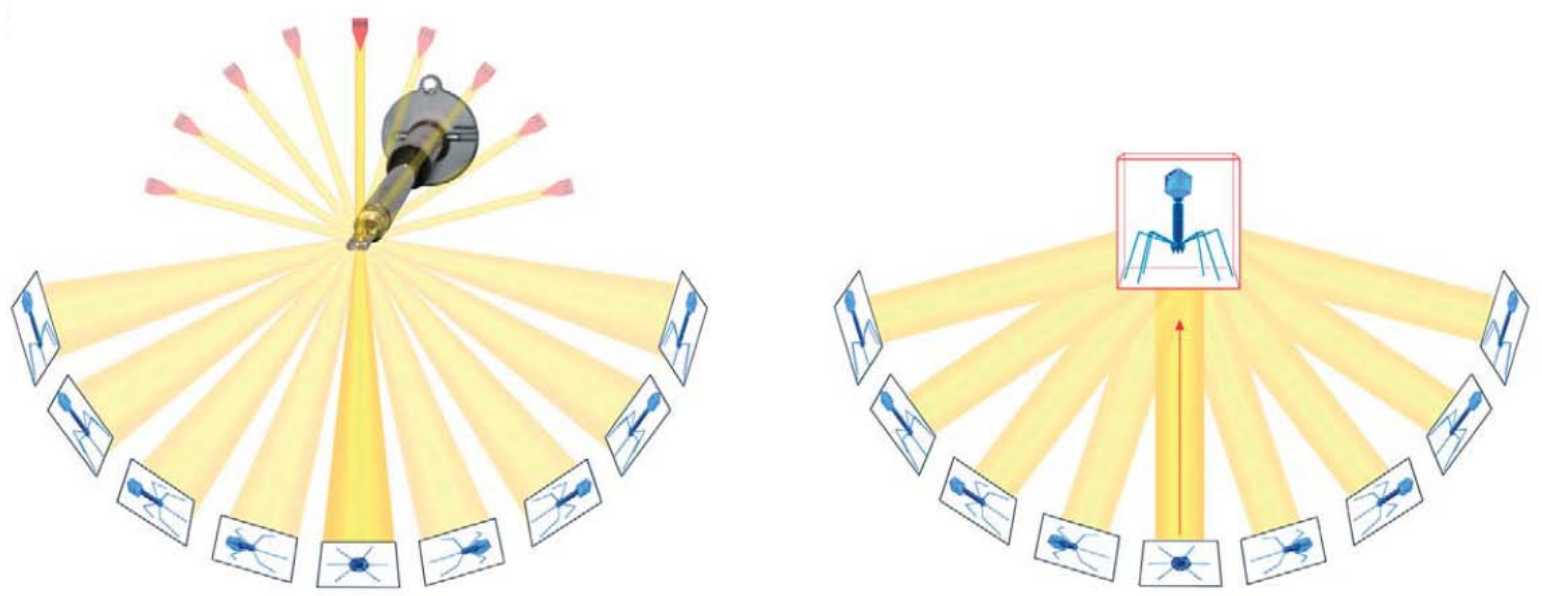

Fig. Il.16 Electron microscopy tomography. Left: The biological specimen is imaged from different angles by tilting the specimen holder in microscope. Right: Computer-aided back-projection of each tilt view is used to reconstruct a 3D volume of the original structure. Reprinted from (McIntosh et al., 2005) with permission from Elsevier.

Employing serial section reconstruction and EM tomography on $C$. elegans fixed via HPF and FS, I attempted to characterize the ultrastructure of NMJs DPs in more detail. The established description should serve as basis upon which defects and phenotypes of $A Z$ morphology in synaptic transmission mutants can be judged. Accordingly, I conducted an ultrastructual analysis of DP morphology and synaptic transmission regulation in mutants involved in AZ assembly, with special focus on SYD-2, an established regulator of AZ formation. 


\section{III.Introduction Chapter 1}

The presynaptic active zone (AZ) and its elaborate network of proteins are crucial for the release and subsequent endocytosis of synaptic vesicles (SV). However, despite their importance for orchestrating SV release, little is known about the ultrastructure of the $A Z$ matrix and its characteristic dense projection (DP). Depending on the type of synapse and the organism, the architecture of these DPs varies in size and structure; possibly reflecting the different functional profiles of these synapses (reviewed in Zhai and Bellen, 2004; also refer to 2.3).

Early electron microscopic (EM) analysis of conventionally fixed C. elegans led to the description of neuromuscular junction (NMJ) DPs as rather simple and plaque like structures (White et al., 1986). The implementation of high-pressure-freeze (HPF) and freeze substitution (FS) in EM preparation now allows reliable preservation of the ultrastructure in a nearly native state in intact animals. Fine structures such as tethers extending from the $A Z$ and between SVs can now be observed (Stigloher et al., 2011). Only recently, EM tomography and the development of computer-aided reconstruction programs for 3D image analysis have provided us with the tools to investigate the architecture of the DP more closely. As initial task of my thesis, I attempted to provide a precise characterization of the wild type NMJ DP ultrastructure by combining HPF sample preparation with serial section reconstruction and EM tomography.

Subsequently I wanted to use this description to identify the function of known $A Z$ proteins in DP assembly. A number of proteins has been described to localize at presynaptic sites and interact with components of the fusion machinery (also refer to 2.2). However, our understanding of how the formation of DPs is controlled is limited. One of the most promising candidates for $A Z$ assembly in $C$. elegans is the scaffolding protein SYD-2, the C. elegans homolog of human Liprin-a (also refer to introduction 2.2.5). It is evolutionarily conserved and genetic data support an upstream regulatory role of SYD-2/Liprin- $\alpha$ in AZ formation by recruiting synaptic proteins to presynaptic sites (Zhen and Jin, 1999, Dai et al., 2006, Patel et al., 2006). An elegant study on the hermaphrodite specific neurons (HSN) in C. elegans has established an assembly hierarchy for AZ components (Patel et al., 2006). Patel and colleagues fluorescently tagged known AZ proteins and expressed them in wild type animals to characterize their localization within the HSN. Then they examined how 
the localization of the same proteins is altered in deletion mutants for the same panel of proteins (Patel et al., 2006). Their genetic analysis revealed a three-layer hierarchy for HSN assembly (Fig. III.1): (1) synaptic localization in HSN is determined by the transmembrane adhesion molecules SYG-2 and its receptor SYG-1. During synaptogenesis they are expressed in vulval epithelial cells and HSN, respectively. Their interaction defines the region for synapse formation. However, SYG-1 and SYG-2 are only required in a subset of neurons and no equivalent in motor neurons and interneurons has yet been identified. (2) Downstream regulators SYnapse Defective 1 (SYD-1) and SYnapse Defective 2 (SYD-2) act together to recruit other presynaptic components and SVs (Fig. III.1). SYD-1 and SYD-2 seem to be ubiquitously involved in synapse formation. The notion that SYD-1 and SYD-2 are key organizing molecules for synaptic assembly is supported by the finding that other AZ proteins as well as SV and DCV proteins fail to localize properly to presynaptic sites in HSN as well as GABAergic motor neurons in the absence of SYD-1 or SYD-2 (Zhen and Jin, 1999; Patel et al., 2006). (3) Apart from the regulation through upstream SYD-1 and SYD-2, the other presynaptic proteins and SV proteins accumulate mostly independent of each other. Loss of any of these downstream proteins does not affect SYD-1 or SYD-2 localization, supporting their upstream function (Patel et al., 2006).

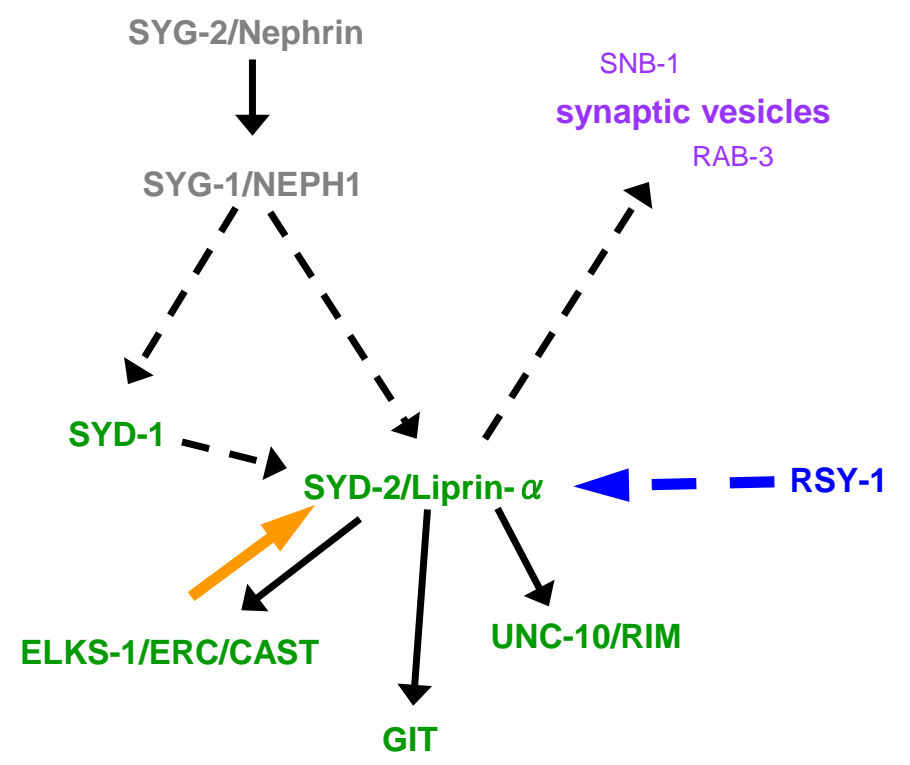

Fig. III.1 Hierarchical assembly of the active zones in C. elegans. The transmembrane adhesion molecules SYG-2 and SYG-1 specify synapse location in the hermaphrodite specific neurons (gray), but not in motor neurons. Downstream regulators SYD-1 and SYD-2 recruit AZ proteins (green) like ELKS, GIT and UNC-10 (see also 2.2) and SV proteins (purple) like SNB-1 and RAB-3 to the 
presynaptic site. RSY-1 has been identified as negative regulator (blue) of SYD-2 (Patel and Shen, 2009). Solid arrows: biochemical interaction; dashed arrows: genetic interaction. Adapted from (Patel et al., 2006).

Yet, precisely how SYD-2 operates is still unknown. Biochemical assays have revealed interaction of the vertebrate SYD-2 homolog Liprin- $\alpha$ with several $A Z$ proteins and the SV transporter kinesin-3 KIF1A/UNC-104 (also refer to 2.2.5). LosS of SYD-2 has been shown to affect DP length in C. elegans (Zhen and Jin, 1999) whereas a gain-of-function (GF) mutation was able to bypass the requirement of upstream regulator SYD-1. However, the ability of SYD-2 GF to promote AZ assembly requires the presence of ELKS (Dai et al., 2006). Recently, a Regulator of SYnapse formation (RSY-1) has been identified to negatively affect SYD-2 function, probably by compromising the binding of SYD-2 and ELKS (Fig. III.1) (Patel and Shen, 2009).

Therefore SYD-2 facilitates a promising candidate and was used on this study to investigate the ultrastructure, assembly and function of presynaptic DPs in synaptic transmission. 


\section{Results Chapter 1}

The triggered release of synaptic vesicles (SV) is tightly regulated by a complex network of proteins that form the active zone (AZ) at presynaptic terminals (reviewed in Dresbach et al., 2001). In the center of each presynaptic AZ, a distinct electron dense projection (DP) can be visualized by electron microscopy (EM). These DPs are surrounded by a cloud of SVs and dense core vesicles (DCV). Despite their importance for regulated SV release, the molecular composition, 3D structure as well as their precise function remains unclear. In this study, I used the model organism Caenorhabditis elegans and applied high-pressure-freeze (HPF) EM in combination with $3 \mathrm{D}$ reconstructions of serial EM thin sections and tomograms to resolve the $3 \mathrm{D}$ architecture of the presynaptic DP.

C. elegans locomotion is controlled by two types of motor neurons, excitatory and inhibitory motor neurons releasing acetylcholine (ACh) and $\gamma$-Aminobutyric acid (GABA) respectively. They innervate the body wall muscles (BWM) of $C$. elegans in an alternating fashion via activation and contralateral inhibition, thus generating the characteristic sinusoidal movement of the worm (McIntire et al., 1993). En passant synapses are formed along the axons of the motor neurons and contact postsynaptic muscle arms extending from adjacent BWMs to form NMJs (White et al., 1986, Dixon and Roy, 2005) (Fig. IV.1). Motor neurons themselves are innervated by a set of command interneurons that receive their input from sensory neurons upon stimulation by environmental cues (de Bono and Maricq, 2005; refer also to 2.4). 


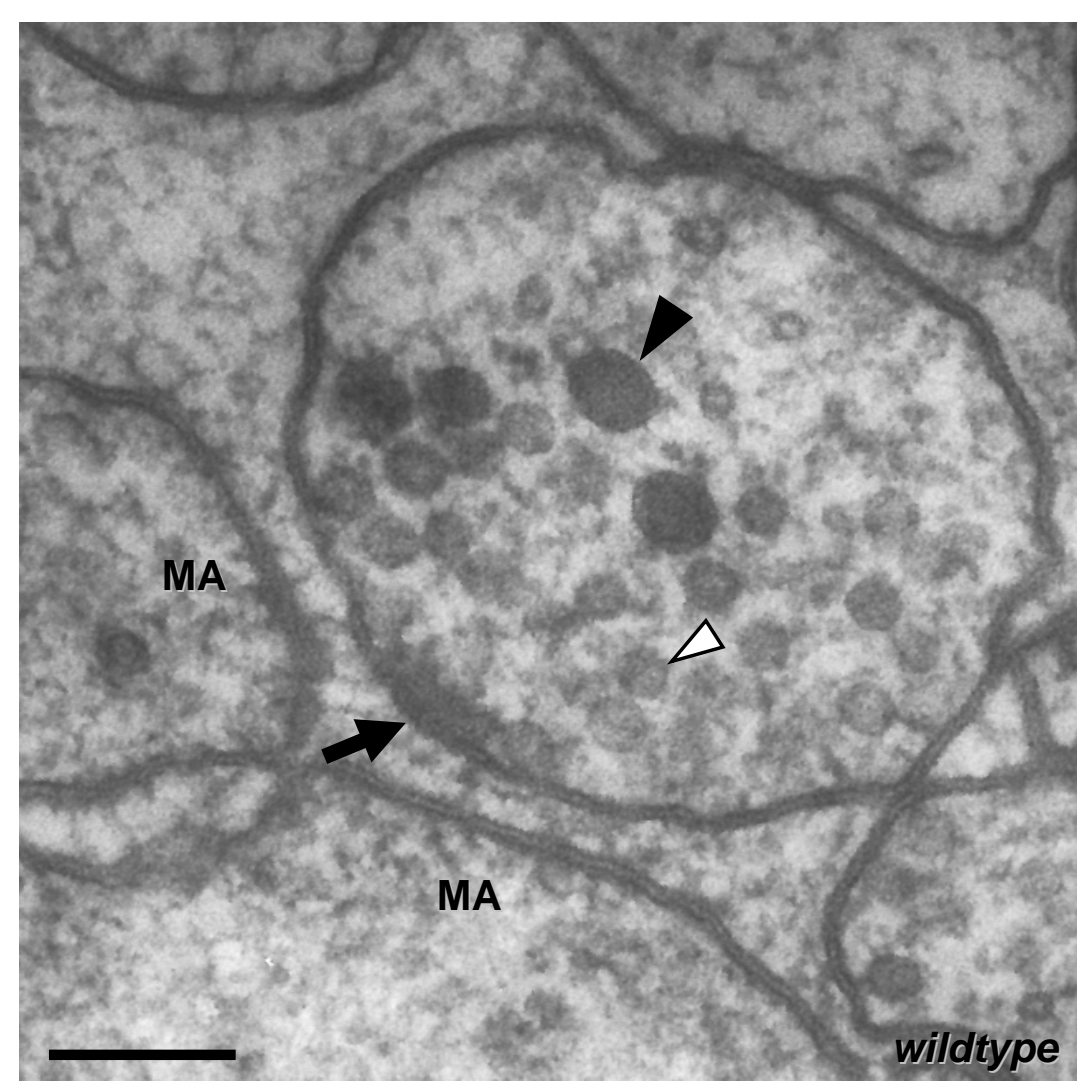

Fig. IV.1 Synapse morphology at neuromuscular junctions. Electron micrograph of a motor neuron synapse. The presynaptic dense projection (arrow), SVs (white arrowhead), DCVs (black arrowhead) and the postsynaptic muscle arm (MA) are indicated. The image was provided by Dr. Jan Hegermann. Scale bar is $100 \mathrm{~nm}$.

The $C$. elegans DP can easily be recognized in EM cross sections and has been described as rather simple plaque-like structures after conventional EM preparation (Fig. II.7) (White et al., 1986; refer also to 2.3; Zhai and Bellen, 2004). However, recent advances in preservation procedures and 3D-image analysis allowed us to obtain ultrastructural details of synapses in high resolution, indicating that the architecture of $C$. elegans DPs is more elaborate than previously thought.

\subsection{D reconstructions reveal the complex structure of presynaptic dense projections in C. elegans.}

Reconstruction from $50 \mathrm{~nm}$ ultrathin serial sections (Fiala, 2005) of worms prepared via HPF and freeze substitution (FS) provided an initial assessment of NMJ DP morphology (refer to 9.3 and 9.6 for more details). Cholinergic as well as GABAergic 
motor neuron DPs are repeatedly observed to split into branches (Fig. IV.2). SVs localize between the dense material, often close or docked at the presynaptic membrane.
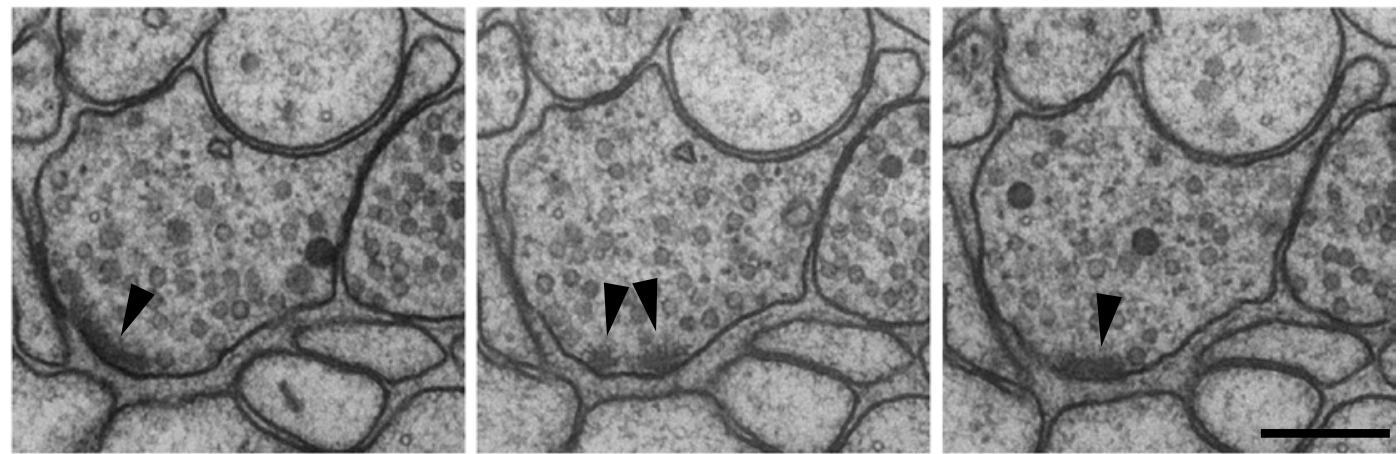

Fig. IV.2 Dense projections of C. elegans NMJs often branch. In serial thin cross sections (50 nm) of wild type C. elegans neuromuscular synapses DPs were regularly observed to branch into two smaller DPs or to fuse in subsequent sections, contradicting the simple ultrastructure described so far (White et al., 1986). Scale bar is $200 \mathrm{~nm}$.

Additionally, fibrous extensions were observed emanating from this core structure, contacting SVs in the vicinity of the DP (Fig. IV.3, left). These so-called tethers are already visible in thin section, but even more so in tomographic reconstructions of the dense projection (Fig. IV.3, right).
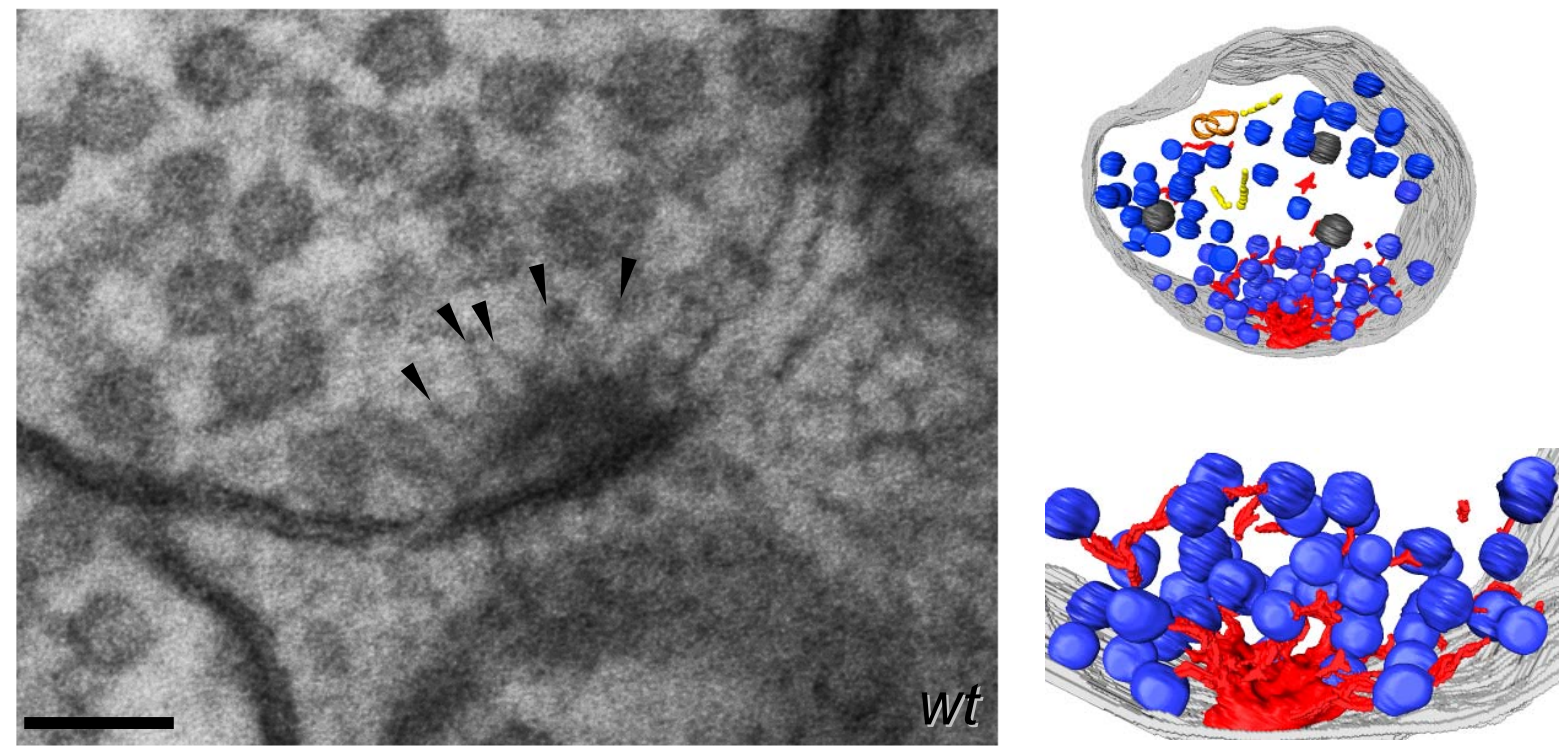

Fig. IV.3 Fibrous extensions extend from the DP contacting adjacent SVs. Improved preservation of synaptic ultrastructure through HPF and FS allowed observation of thin tethers emanating from the DP into the synaptic terminal. SVs are often found in close contact with these tethers. Left: $50 \mathrm{~nm}$ thin 
section: arrowheads point at tethers. Scale bar is $50 \mathrm{~nm}$. Right: (top) 3D volume segmentation of a tomographic reconstruction acquired from a $250 \mathrm{~nm}$ section. Synaptic terminal with membrane (gray), microtubules (yellow), DP and emanating tethers (red), SVs (blue) and DCVs (black). (Bottom) enlarged view of the same DP and tethers with surrounding SVs. The segmented tomogram was provided by Dr. Jan Hegermann.

To better assess DP morphology, I generated longitudinal thin sections parallel to the AZ membrane that allow a top view onto the DP (Fig. IV.4). It became apparent from these sections, that NMJ DPs exhibit one or more branch points from which small branches extend into the $X$ and $Y$ plane in a rather symmetrical fashion. Between these branches, bay-like slots (termed "bays" below) are formed in which SVs were frequently found close to the presynaptic plasma membrane (Fig. IV.4).

\section{physical thin sections}
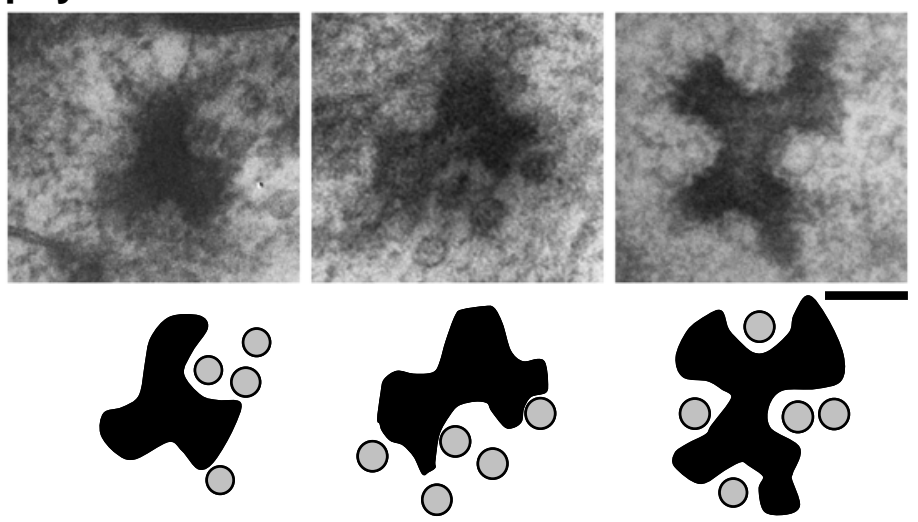

electron tomography
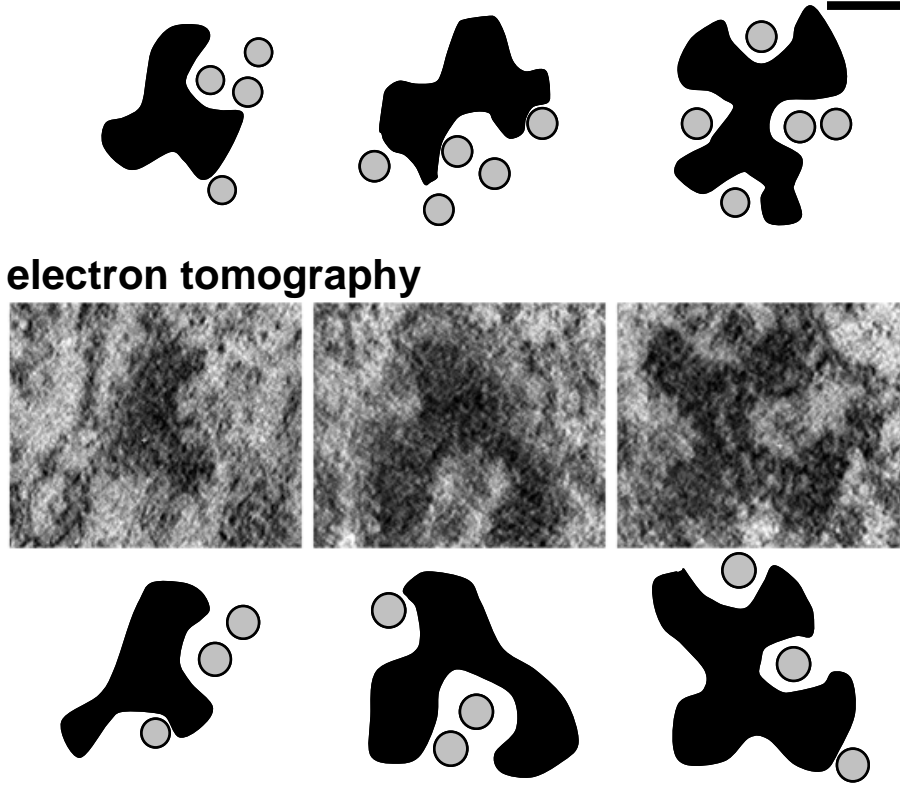

Fig. IV.4 Branched morphology of the NMJ DP was verified by longitudinal thin sections and tomography. Top: $50 \mathrm{~nm}$ longitudinal thin sections. In sections parallel to the presynaptic membrane, the branched architecture of NMJ DPs is clearly visible. Schematic representations are shown below each image: DP (black), SVs (gray). SVs localize in the bays formed by the branches. Scale bar is 100 $\mathrm{nm}$. Bottom: Projection from a tomographic reconstruction of a $300 \mathrm{~nm}$ thick section. EM tomography confirmed the branched DP structure observed in longitudinal sections. Schematic representations are 
shown below. Tomograms and tomographic projections were provided by Alexandr Goncharov, thin sections were provided by Dr. Jan Hegermann and the author.

Thin section images from this viewpoint were challenging to obtain, as acquisition relied on the low probability of sectioning the synapse in the appropriate orientation. Tomographic reconstructions, however, allow rotation in any direction and are relatively easy to obtain. I collected double tilt series from thick cross sections (250$300 \mathrm{~nm}$ ) tilted from $-60^{\circ}$ to $+60^{\circ}$ with $1^{\circ}$ increment and reconstructed the volume via back projection using IMOD (Kremer et al., 1996). The IMOD Slicer window allows tilting of the reconstructed volume at the desired angle. Several projections similar to top views onto the DP were obtained and resembled the same DP architecture observed in longitudinal sections (Fig. IV.4). Again, SVs were frequently observed close to the plasma membrane within the bays formed at DP branch points.

Cellular components such as the endoplasmatic reticulum (ER), microtubules are clearly visible in tomograms. Importantly, the distribution of SVs and DCVs relative to the DP are clearly visible in the tomograms (Fig. IV.3). In agreement with a previous analysis of wild type C. elegans synapses (Stigloher et al., 2011), these reconstructions confirmed the presence of an elaborate network of tethers between SVs as well as between SVs and the DP (Fig. IV.3).

The complex morphology of DPs in excitatory as well as inhibitory motor neurons was verified by volume segmentation of tomographic reconstructions. (Fig. IV.5). Compared to cholinergic synapses, GABAergic synaptic terminals are usually larger and often contain two separate DPs per terminal. However, the size of GABAergic DP is similar to that of cholinergic synapse DPs (data not shown). 
cholinergic
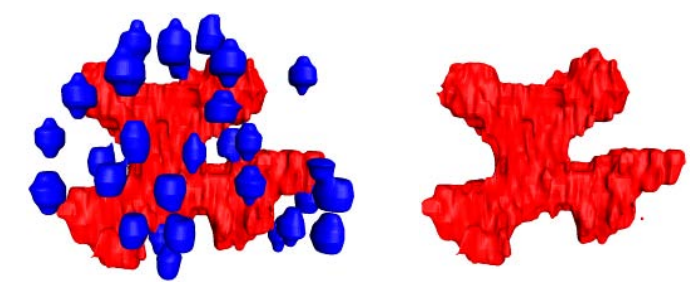

GABAergic
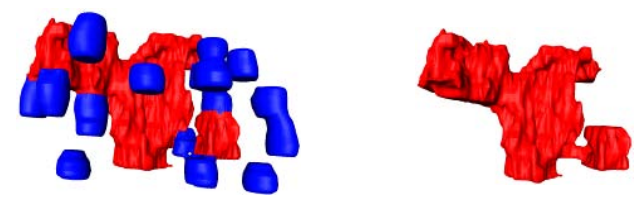

Fig. IV.5 Architecture of GABAergic and cholinergic synapse DPs are similar. EM tomography revealed that GABAergic and cholinergic synapses exhibit the same branched morphology with SVs localizing in the bays between the branches. The cholinergic DP tomogram was provided by Alexandr Goncharov.

Based on this analysis, I hypothesized that proteins required for normal DP morphology will play important roles in SV recruitment and consequently in synaptic transmission. Because of the frequent observation of SVs in the DP bays, I propose these bays be designated fusion sites.

Longitudinal thin sections of neuron-neuron synapses in the ventral nerve cord revealed a similarly symmetric organization of DPs as in NMJs (Fig. IV.6). Some synapses exhibit considerably larger DPs with accordingly more branch points and bays. Yet, the characteristic branch points are clearly recognizable. As expected, SVs were found within the bays close to the plasma membrane.

The high similarity in structural design between NMJ and neuron-neuron DPs indicates the existence of a defined subunit from which these structures are build, thus creating a regular pattern of branches and bays. 


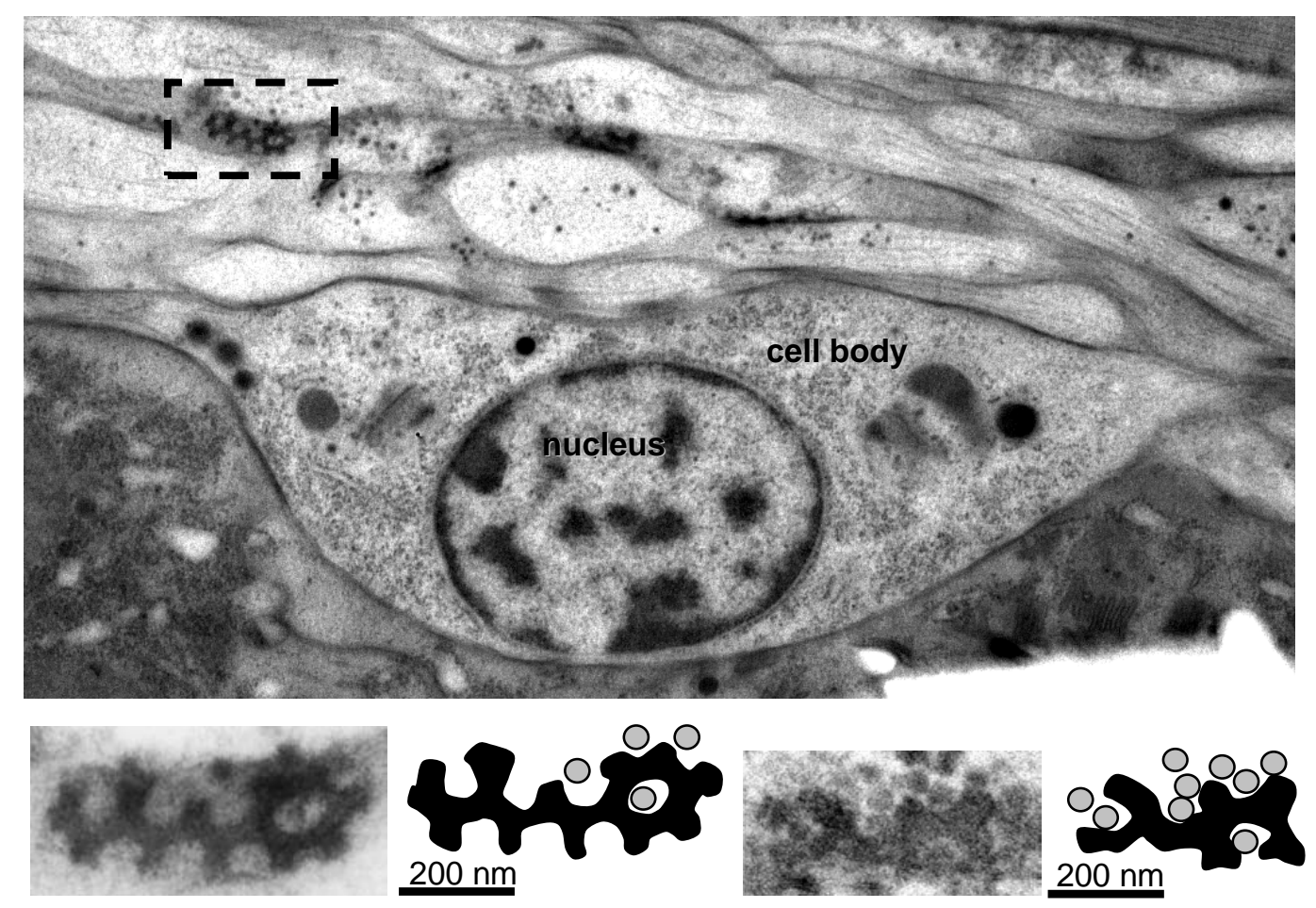

Fig. IV.6 Neuron-neuron synapse DPs have a similar geometric organization as NMJ DPs. Longitudinal thin sections parallel to the presynaptic membrane of neuron-neuron synapses in the ventral nerve cord. Enlarged image of the boxed DP is shown below. Long neuron-neuron synapse DPs have a similarly complex morphology with regularly spaced branches as NMJ DPs. SVs localize frequently in the bays. Schematic representations are shown to the right. Overview image and left thin section were provided by Dr. Jan Hegermann.

\subsection{The active zone protein SYD-2 regulates the size of DPs and affects proper vesicle localization at presynaptic terminals.}

Having resolved the 3D architecture of presynaptic DPs in C. elegans, I was interested in understanding which proteins form the DPs, how they assemble and what their function in synaptic transmission is. Therefore, I analyzed mutants which lack proteins involved in synaptogenesis and reconstructed their DPs from EM serial sections and tomograms.

The C. elegans Liprin- $\alpha$ homolog SYD-2 has been implicated in the recruitment of AZ proteins to presynaptic DPs, probably via interactions mediated by its coiled coil and sterile alpha motif (SAM) domains (Fig. IV.7). Most synaptic proteins fail to localize to synaptic sites in syd-2 LF mutants, indicating that it is an important organizer of DP morphology (Zhen and Jin, 1999; Patel et al., 2006). 

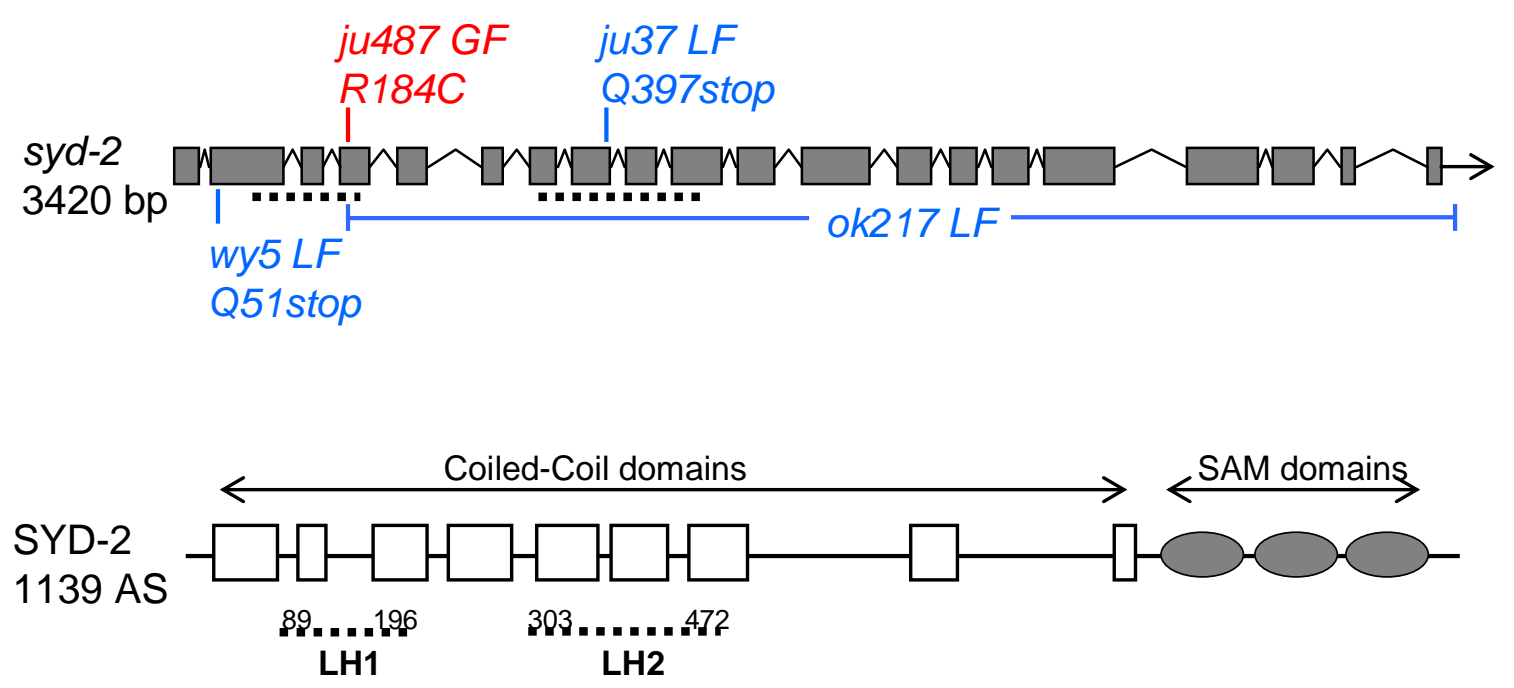

Fig. IV.7 syd-2 gene and SYD-2 protein structure. Schematic of the syd-2 gene structure indicating loss-of-function mutations (blue) and the gain-of-function mutation (red) used in this study. SYD-2 is the C. elegans homolog of mammalian Liprin- $\alpha$ and is composed of $\mathrm{N}$-terminal coiled coil domains and three C-terminal SAM domains. Adapted from (Taru and Jin, 2011). Two Liprin Homology domains (LH1 and LH2) were identified within the coiled coil domains, indicated by dotted lines (Serra-Pages et al., 1998, Taru and Jin, 2011).

Previously, motor neuron DPs of a $C$. elegans syd-2 LF mutants prepared by conventional chemical fixation have been reported to show alterations in DP length (Zhen and Jin, 1999). Furthermore, synapses of the hermaphrodite specific neurons (HSN) in the same syd-2 allele fail to recruit fluorescently-tagged synaptic markers (Dai et al., 2006, Patel et al., 2006).

Recently, the function of highly conserved coiled coil segments in the SYD-2 Nterminus (Liprin 브omology domains LH1 and LH2; Fig. IV.7) (Schoch et al., 2002) has been described more closely. LH1 was found to be crucial for SYD-2 dimer formation and could suppress the syd-1 LF phenotype in HSN synapses (Taru and Jin, 2011).

These studies indicate that SYD-2 plays an important role in synapse assembly and/or stability. To investigate the influence of SYD-2 on NMJ DP architecture in C. elegans, I took advantage of available syd-2 LF (allele ju37, ok217 and wy5) and gain-of-function (GF; allele ju487) mutants (Fig. IV.7). The syd-2(ju487) GF mutant exhibits a R184C amino acid exchange in the highly conserved coiled coil $\mathrm{N}$ terminus. Enhanced activity of SYD-2 R184C was sufficient to bypass the requirement of upstream SYD-1 to promote synapse formation (Dai et al., 2006). 
Additionally, our collaborator Ya Dai has generated a strain that overexpresses a transgene containing the GF protein SYD-2 R184C in the syd-2(ju487) GF background (syd-2(ju487); juls204)). This mutant was termed syd-2 super-gain-offunction (super-GF). Western blot analysis of worm lysates clearly showed the overexpression of SYD-2 R184C in the syd-2 super-GF mutant compared to syd-2 GF mutant or wild type levels of SYD-2 (Fig. IV.8). The truncated SYD-2 protein in syd-2 LF mutants was not detected as the according part of the blot was used for the actin loading control.

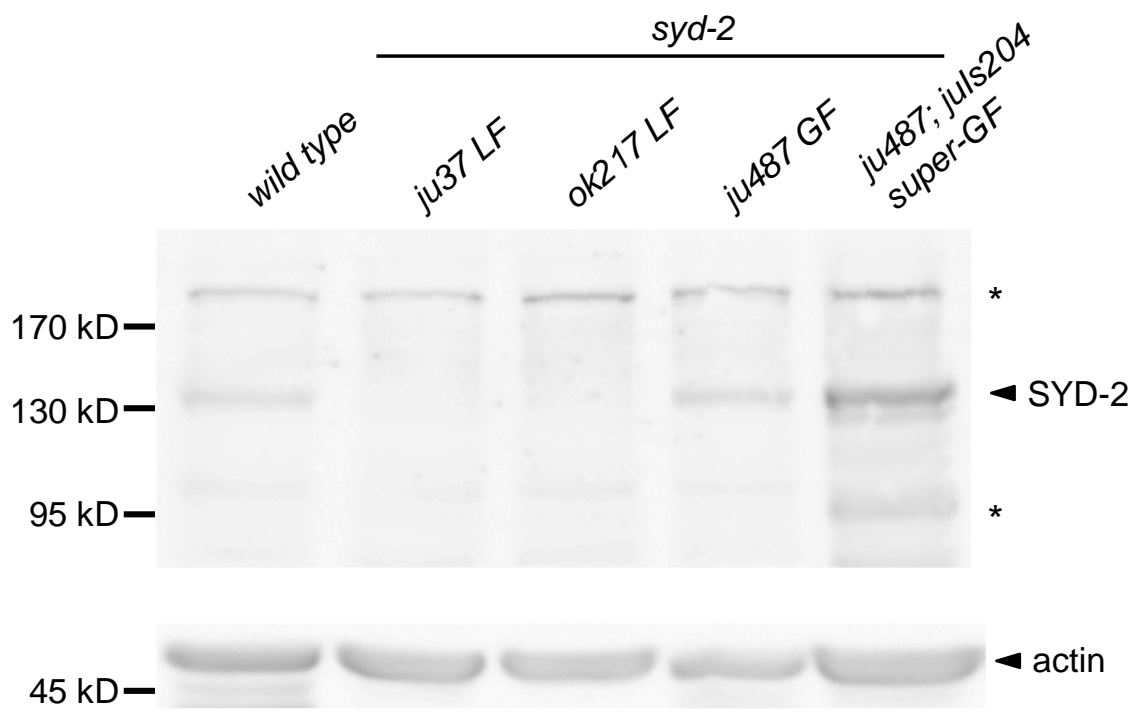

Fig. IV.8 Western blot analysis following SDS-PAGE of worm lysates. Overexpression of SYD-2 R184C protein in the syd-2 super-GF ((ju487); nuls204) mutant was detectable in Western blot of total worm lysates. The polyclonal antibody against the N-terminus of $C$. elegans SYD-2 recognizes full length SYD-2 (130 kDa) in wild type as well as full length SYD-2 R184C in syd-2 GF (ju487) and syd-2 super-GF ((ju487); juls204) mutants. Overexpression of SYD-2 R184C protein in the syd-2 super-GF mutant could be verified by a stronger band compared to wild type and syd-2 GF. As expected, no full length SYD-2 was detectable in the syd-2 LF mutant alleles ok217 and ju37. Actin was used as loading control. Asterisks mark unspecific bands.

As described by Zhen and Jin (Zhen and Jin, 1999), the syd-2 (ju37) LF mutation leads to an amber stop in exon 8 (aa 397) while syd-2 (ok217) exhibits a large deletion disrupting the gene from exon 4 to 12 (personal communication Hidenori Taru). The sequence upstream of the deletion of syd-2(ok217) contains a missense mutation leading to an ochre stop at aa 200, truncating SYD-2 just after the LH1 domain (Wagner et al., 2009) (Fig. IV.7). LF allele wy5 exhibits a point mutation 
leading to an early stop at aa 51 at the 5' end of exon 2 and was proposed to be a syd-2 null (Patel et al., 2006; personal communication Kang Shen).

Consistent with previous findings, my analysis of $50 \mathrm{~nm}$ electron micrographs of syd2 LF mutants showed no alteration of DP width (data not shown) (Zhen and Jin, 1999). However, the length of the DPs in the longitudinal axis as calculated from serial sections was significantly reduced. (Fig. IV.9). In worms prepared via HPF and FS, wild type DPs ranged between 100 and $350 \mathrm{~nm}(181 \pm 9.02)$, while syd-2 LF mutant alleles ju37 and ok217 showed a tendency toward shorter DPs ranging from 50 to $250 \mathrm{~nm}$ (117.6 \pm 8.92 and $128.8 \pm 8.18$, respectively) (Fig. IV.9). It is possible that syd-2(ju37) and syd-2(ok217) produce truncated proteins that have a dominant negative effect, interfering with proper DP assembly. In fact, the presence of truncated SYD-2 protein products have previously been demonstrated via Western blot in syd-2 (ju37) mutants but not in syd-2(ok217) (Wagner et al., 2009).

Initial analysis of the null allele wy5 revealed a DP size ranging from 50 to $300 \mathrm{~nm}$ (148.8 \pm 8.64). The DP phenotype is less severe compared to ju37 and ok217 alleles. I have not yet performed SDS-PAGE and Western blot analysis of syd-2(wy5) worm lysate to confirm the absence of SYD-2 protein in the wy5 allele. It is conceivable that some read through product is translated despite the stop codon, or that translation started from a downstream ATG and resulted in a functional protein. The wy5 allele was not included in further experiments of this study.

Together with the published data on SYD-2/Liprin- $\alpha$ function in recruiting AZ proteins (Zhen and Jin, 1999, Dai et al., 2006, Patel et al., 2006), our observations indicate that SYD-2 is required for normal DP assembly. If that is the case, the syd-2 GF mutation (Dai et al., 2006) should have opposite effects to those of LF mutants and promote DP formation. Consistent with this prediction, syd-2(ju487) mutants exhibit wild type-like DPs as well as strongly elongated DPs up to $650 \mathrm{~nm}$ (250 \pm 17.75$)$. (Fig. IV.9).

Surprisingly, the syd-2 super-GF mutants did not show a similar phenotype, but rather DPs of wild type length $(212.3 \pm 10.93)$. 


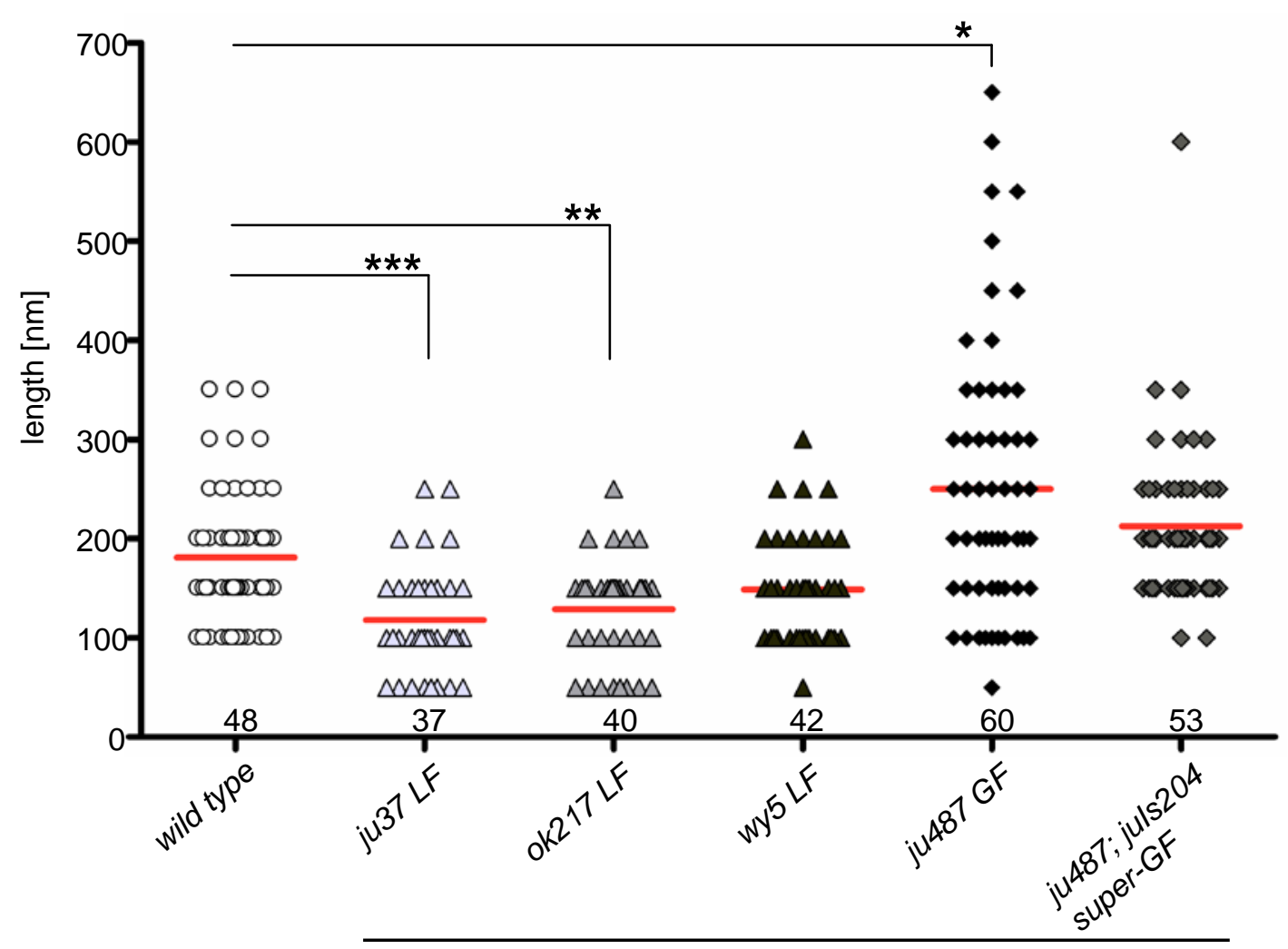

syd-2

Fig. IV.9 DP length of NMJs is affected in syd-2 mutants. DP length along axons was analyzed from $50 \mathrm{~nm}$ serial cross sections. Syd-2 LF mutants (ok217 and ju37) show significantly shorter DPs compared to wild type. In contrast, syd-2(ju487) GF mutants exhibit significantly elongated DPs up to $650 \mathrm{~nm}$. The syd-2 super-GF mutants typically do not show elongated DPs. The mean is indicated by the red line. $\mathrm{N}$ is indicated below data points. Statistics: Kruska-Wallis test and Dunn's multiple comparison test. $\left({ }^{*} \leq 0.05 ;{ }^{* *} \leq 0.01 ;{ }^{* *} \leq 0.001\right)$.

To analyze the effects of SYD-2 on the NMJ DP ultrastructure, 3D reconstructions from serial sections and tomograms of syd-2 LF and GF mutants were generated. Serial section reconstructions of synaptic terminals demonstrate the reduced size of the DP and reduced number of SVs surrounding the DP in syd-2 LF mutants (Fig. IV.10). Reconstructions of the syd-2 GF DP clearly show the enhanced size and the conserved branched morphology (Fig. IV.10). Elongation of the DP seems to be accompanied by the formation of more branch points and bays, similar to the long neuron-neuron DPs (Fig. IV.6). Yet, excessive expression of SYD-2 R184C protein in the syd-2 super-GF mutant does not seem to enhance the elongation of DPs, suggesting that other limiting factors might constrain the effect of SYD-2 super-GF. 
Tomographic reconstructions of NMJ DPs indicate that the bays are maintained in syd-2 mutants (Fig. IV.10). Clear DP branch points were difficult to identify in syd-2 LF mutants due to the reduced DP size. Nevertheless, a small bay structure was preserved in which SVs localize and contact the presynaptic membrane. DP morphology in syd-2 GF and super-GF mutants was wild type-like in terms of branch points and bays.

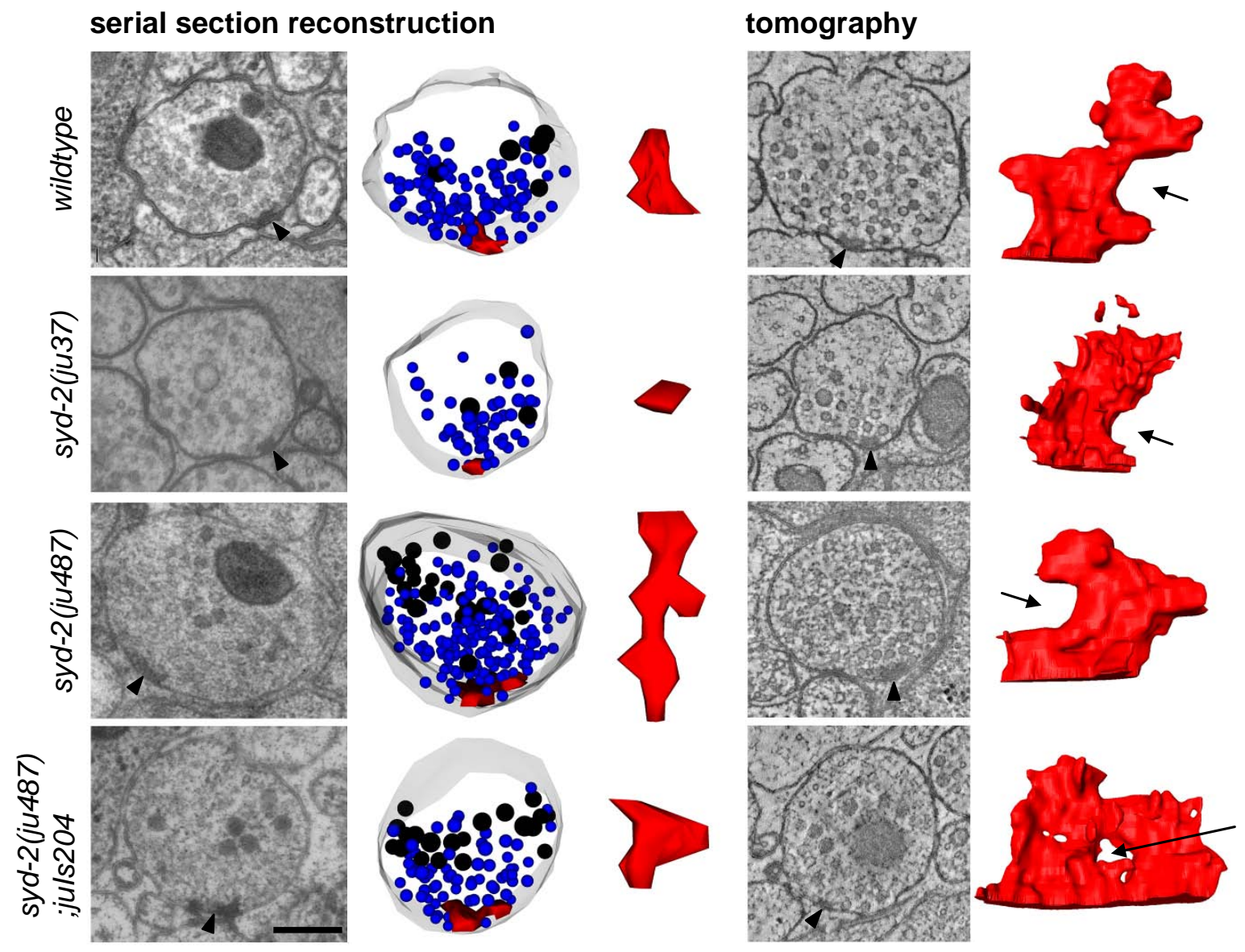

Fig. IV.10 3D reconstructions of NMJ DPs indicate the preservation of branched morphology in syd-2 mutants. Serial section reconstructions illustrate the size of NMJ DPs and the clustering of SVs and DCVs around the DP. A thin section (50) $\mathrm{nm}$ and the corresponding reconstruction of the synaptic terminal and a top view of the DP alone is shown for wild type and syd-2 LF mutants (ju37 and ok217) and syd-2 GF and super-GF mutants (ju487 and ju487; juls204).DPs (red), SVs (blue) and DCVs (black) were reconstructed. Scale bar is $200 \mathrm{~nm}$. Compared to wild type, syd-2 LF mutants have smaller NMJ DPs, while NMJ DPs in syd-2 GF mutant animals are rather elongated with more bays.

Tomographic reconstructions reveal precise the 3D structure of NMJ DPs. Projection from a reconstructed volume of a $250 \mathrm{~nm}$ thick section and the corresponding 3D trace of the DP (red) are shown for wild type, syd-2 LF mutants and syd-2 GF and super-GF mutants. The branched structure is largely maintained in syd-2 mutants, although the LF mutants show decreased complexity due to 
reduced size of the DPs. Arrows highlight a typical bay of the DP. Sample preparation and reconstruction was done by Dr. Jan Hegermann and the author.

To gain a more complete impression of motor neuron morphology in syd-2 mutants, cholinergic and GABAergic neurons were reconstructed within $10 \mu \mathrm{m}$ of ventral nerve cord by serial reconstructions of $50 \mathrm{~nm}$ thin sections. Not only the DP length but also the overall frequency of DPs was reduced in syd-2 LF mutants (Fig. IV.11).

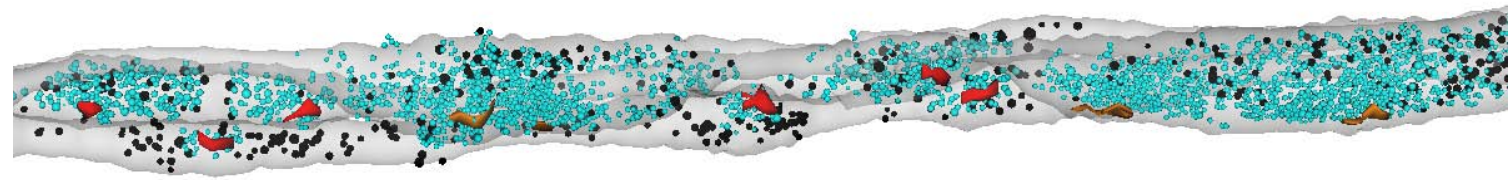

wild type

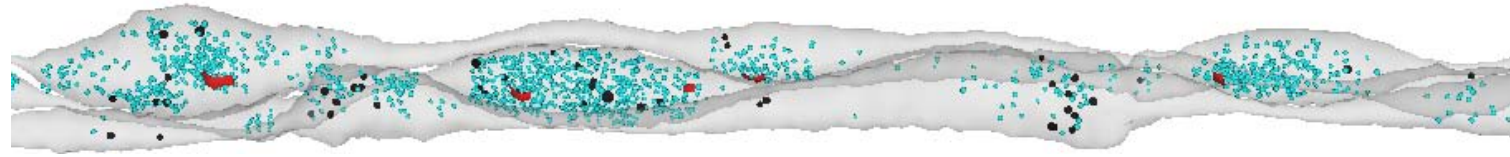

syd-2 (ju37) LF

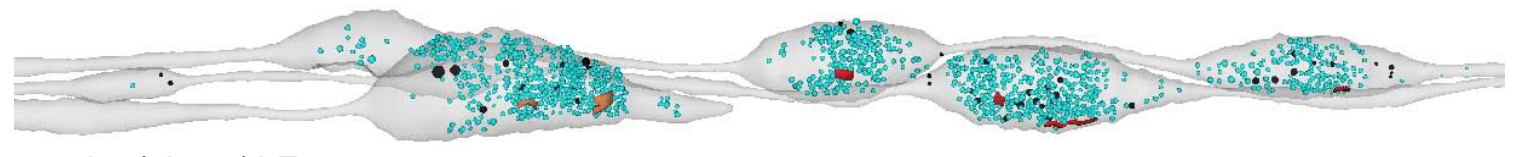

syd-2 (ok217) LF
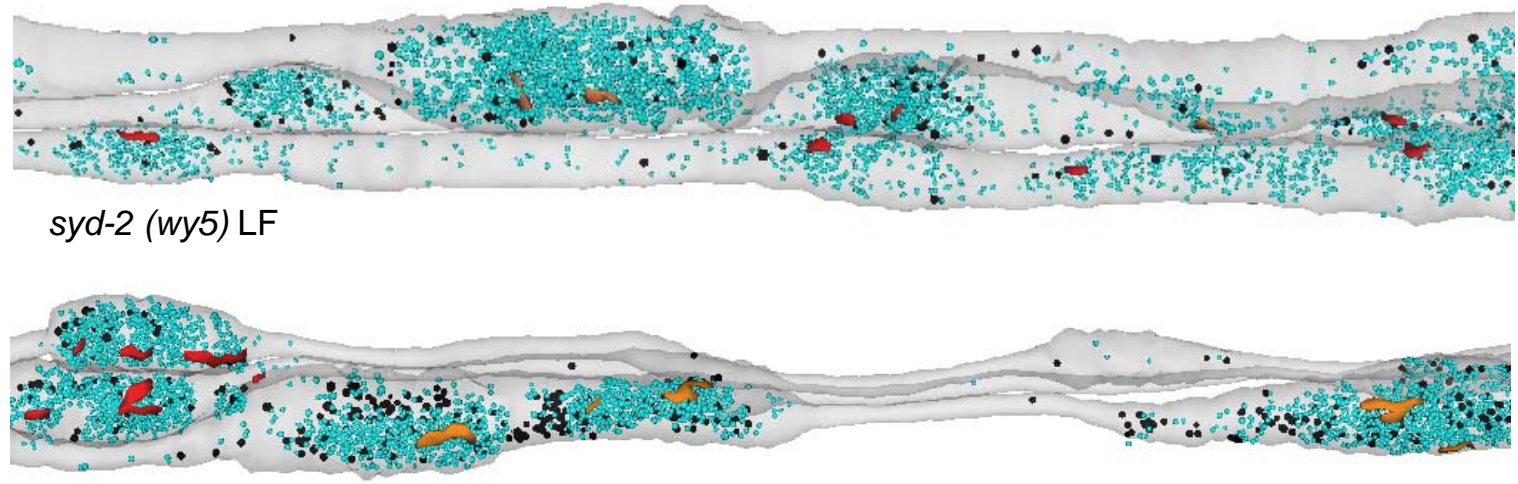

syd-2 (ju487) GF

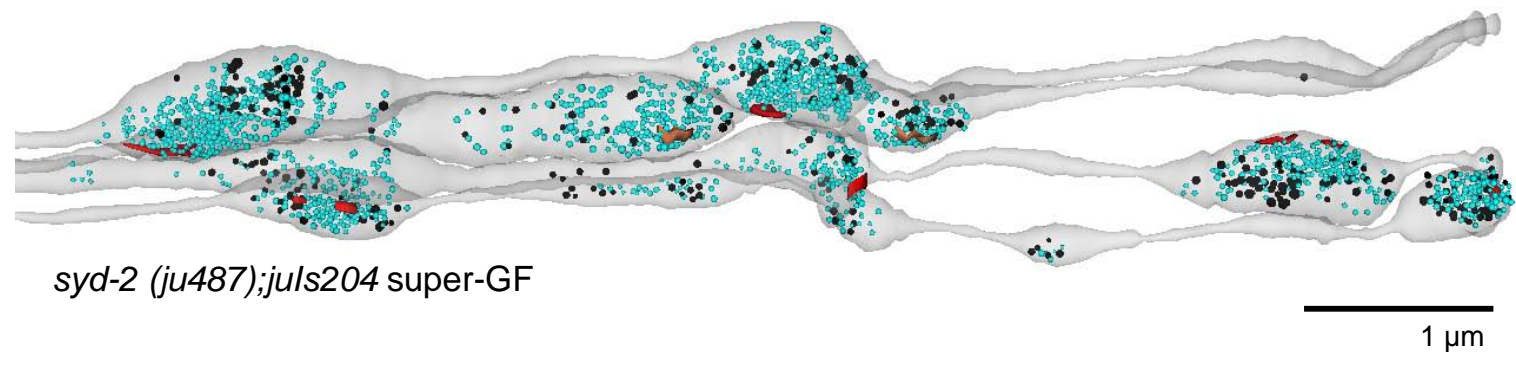

Fig. IV.11 3D Reconstructions of GABA- and cholinergic motor neurons in the ventral nerve cord of wild type and syd-2 mutants. Axon membranes (gray), cholinergic neuron DPs (red), GABAergic neuron DPs (orange), SVs (light blue) and DCVs (black) were reconstructed over $\sim 8 \mu \mathrm{m}$. 
In wild type animals, DPs are surrounded by a SV cloud and few DCVs. The loss of SYD-2 in syd2(ju37) and syd-2(ok217) mutants leads to shorter and less frequent DPs and a reduction in SVs and DCVs. Gain-of-function syd-2(ju487) mutants display elongated DPs in NMJs and normal vesicle localization. Overexpression of SYD-2 R184C in syd-2(ju487); juls204 mutants does not lead to elongated but rather wild type-like DPs. Sample preparation and reconstruction was done by Dr. Jan Hegermann and the author.

In line with previous reports that SYD-2 recruits SV proteins to synaptic sites, serial reconstructions of single synapses (Fig. IV.10) and nerve cords (Fig. IV.11) revealed smaller SV clouds in syd-2 LF mutants (Zhen and Jin, 1999, Dai et al., 2006, Patel et al., 2006). Quantification from EM profiles with visible DP confirmed significantly less SVs and DCVs at synaptic terminals of syd-2 LF mutants (Fig. IV.12 ). In GF and super-GF mutants, the localization of SVs is unaltered compared to wild type ( Fig. IV.10; Fig. IV.11; Fig. IV.12 ).
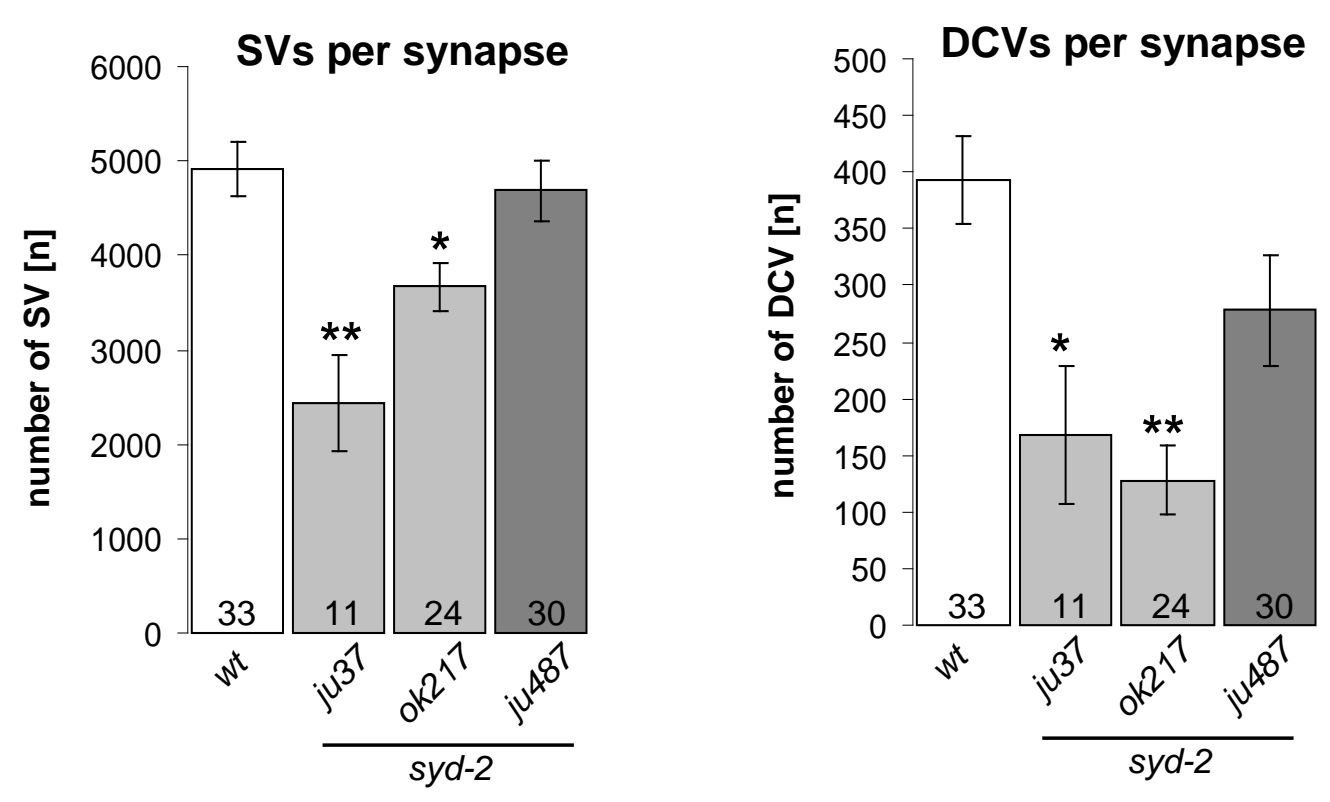

Fig. IV.12 Less SVs and DCVs are localized at NMJ DPs in syd-2 LF mutants. The numbers of SVs and DCVs in wild type and syd-2 mutants were counted in sections with the DP visible and was normalized to the size of the synaptic terminal. LF syd-2 mutants have significantly reduced numbers of SVs and DVCs at NMJs. Error bars represent S.E.M. Statistics: Kruska-Wallis test and Dunn's multiple comparison test. $\left({ }^{*} \leq 0.05 ;{ }^{* *} \leq 0.01 ;{ }^{* * *} \leq 0.001\right)$.

These ultrastructural observations could be verified by analysis of the fluorescentlytagged SV markers synaptobrevin (SNB) and RAB-3 in cholinergic DA and DB (Fig. 
IV.13) and GABAergic DD (Fig. IV.14) motor neurons in the dorsal nerve cord. Each fluorescence punctum along the axon represents the SV cloud in a presynaptic terminal. The overall fluorescence intensity is generally diminished in syd-2 LF mutants and the number of detectable puncta is reduced, suggesting that less SVs are localized at the presynaptic terminals. Again, syd-2 GF and syd-2 super-GF mutants show no obvious phenotype.
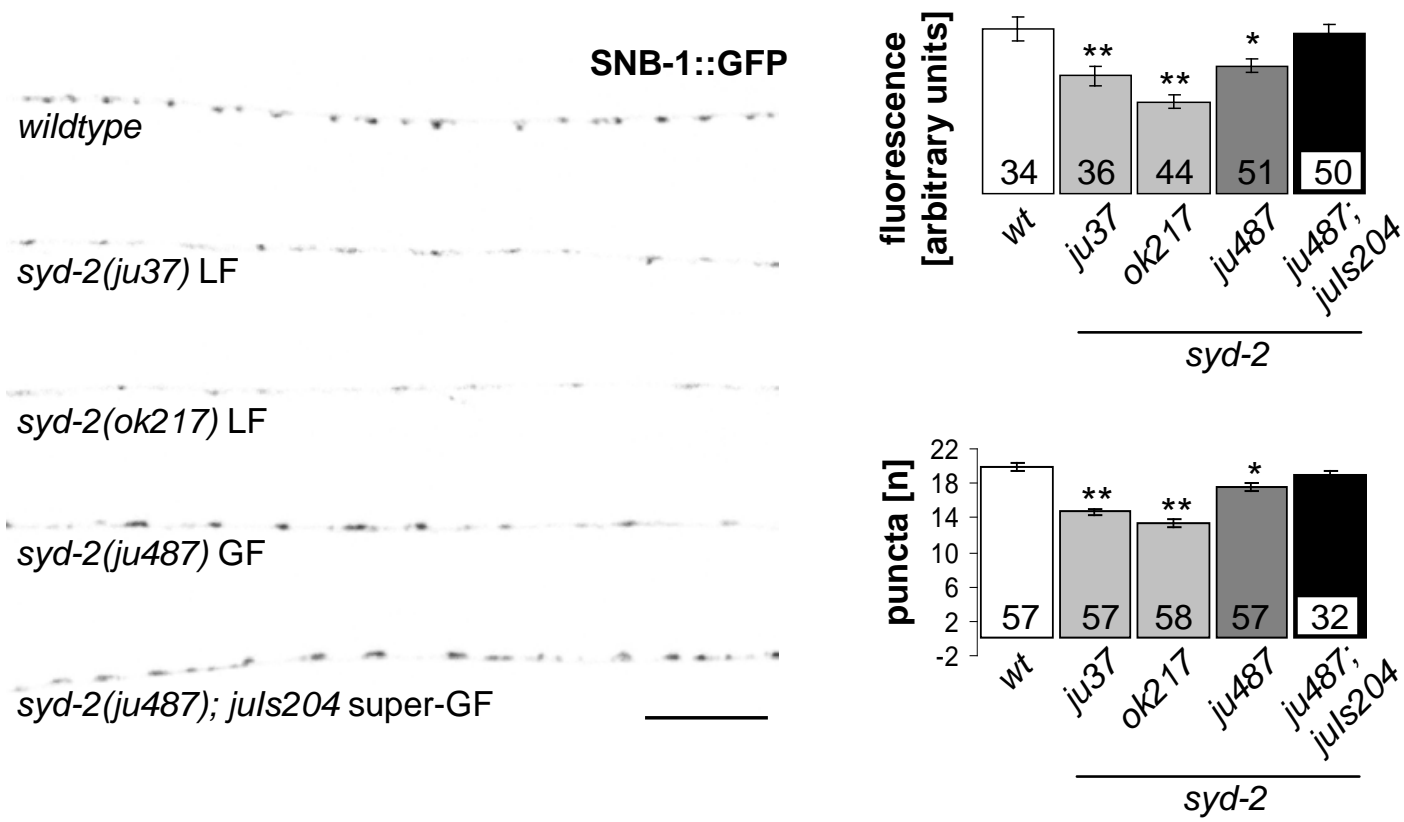

RAB-3::VENUS

wildtype

syd-2(ju37) LF

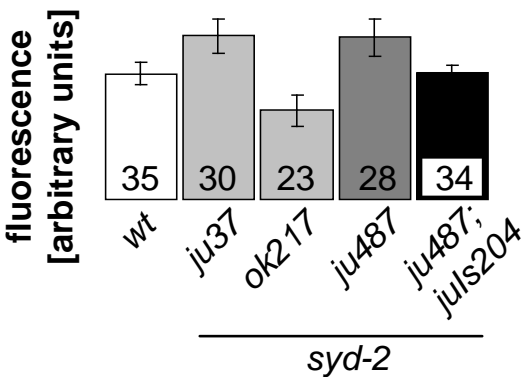

syd-2(ok217) LF

syd-2(ju487) GF

syd-2(ju487); juls204 super-GF

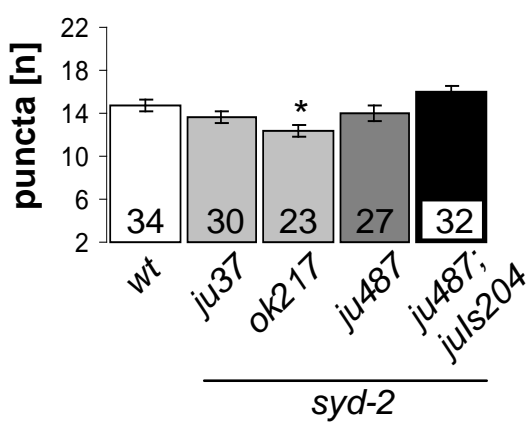

Fig. IV.13 Synaptic vesicle localization is altered at cholinergic NMJs in syd-2 LF mutants. Fluorescence quantification of SV marker proteins SNB-1::GFP and RAB-3::VENUS in wild type and syd-2 mutant strains reveals defects in the axonal distribution of SVs in cholinergic DA and DB motor 
neurons. Total fluorescence and the number of synaptic puncta are generally reduced in syd-2 LF mutants but are wild type-like in syd-2 GF and syd-2 super-GF mutants. Scale bar is $10 \mu \mathrm{m}$. Error bars represent S.E.M. Statistics: ANOVA and Dunnett's post-test $\left({ }^{*} \leq 0.05 ;{ }^{* *} \leq 0.01 ;{ }^{* *} \leq 0.001\right)$.

SNB-1::GFP

wildtype

syd-2(ju37) LF

syd-2(ok217) LF

syd-2(ju487) GF

syd-2(ju487); juls204 super-GF
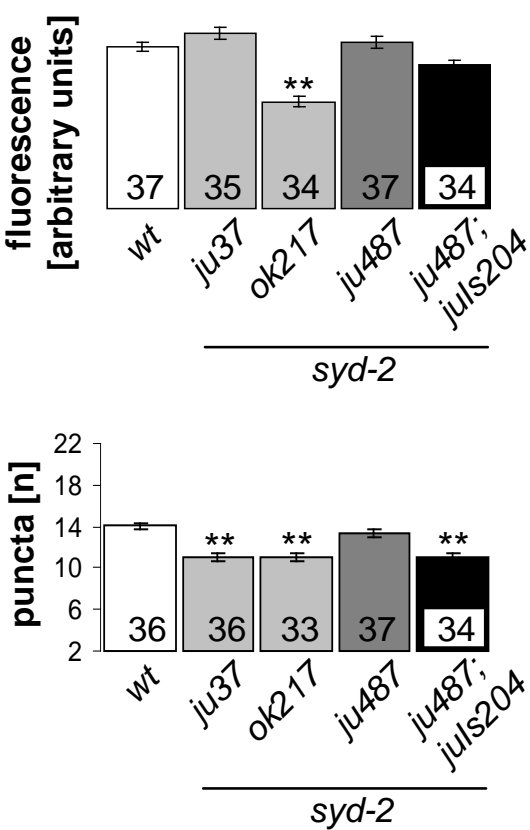

syd-2(ok217) LF

RAB-3::mCherry

wildtype

syd-2(ju37) LF

syd-2(ju487) GF

syd-2(ju487); juls204 super-GF

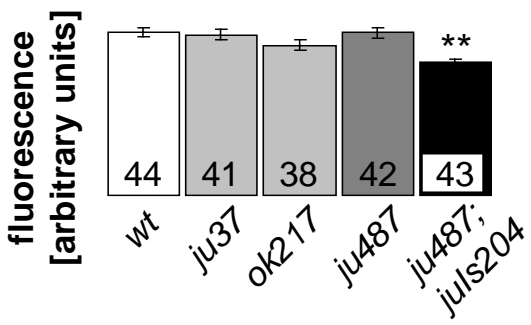

syd-2

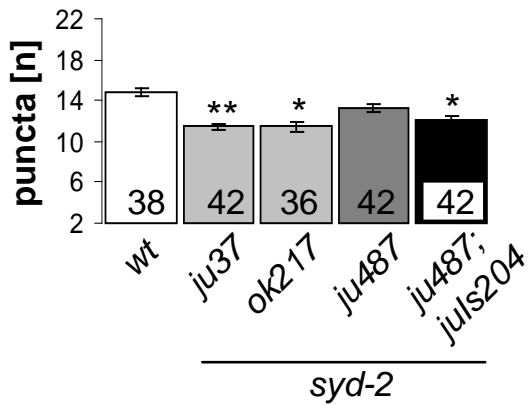

Fig. IV.14 Synaptic vesicle localization is altered at GABAergic NMJs in syd-2 LF mutants. Fluorescence quantification of SV marker proteins SNB-1::GFP and RAB-3::mCherry in wild type and syd-2 mutant strains reveals defects in the axonal distribution of SVs in GABAergic DD motor neurons. Total fluorescence and the number of synaptic puncta are generally reduced in syd-2 LF mutants but 
are wild type-like in syd-2 GF and syd-2 super-GF mutants. Scale bar is $10 \mu \mathrm{m}$. Error bars represent S.E.M. Statistics: ANOVA and Dunnett's post-test $\left({ }^{*} \leq 0.05 ;{ }^{* *} \leq 0.01 ;{ }^{* * *} \leq 0.001\right)$.

As previously shown, the AZ protein RIM-1/UNC-10 localizes to presynaptic sites despite the absence of SYD-2 in syd-2 LF mutants, but puncta are more irregular and unevenly spaced (Deken et al., 2005, Dai et al., 2006). Consistent with these findings, I observed GFP-tagged RIM-1 puncta with slightly reduced fluorescence in cholinergic DA and DB motor neurons in syd-2 LF (Fig. IV.15). The localization in distinct puncta was wild type-like. Reduced fluorescence may be caused by improper integration of RIM-1 into the AZ scaffold in the absence of SYD-2.

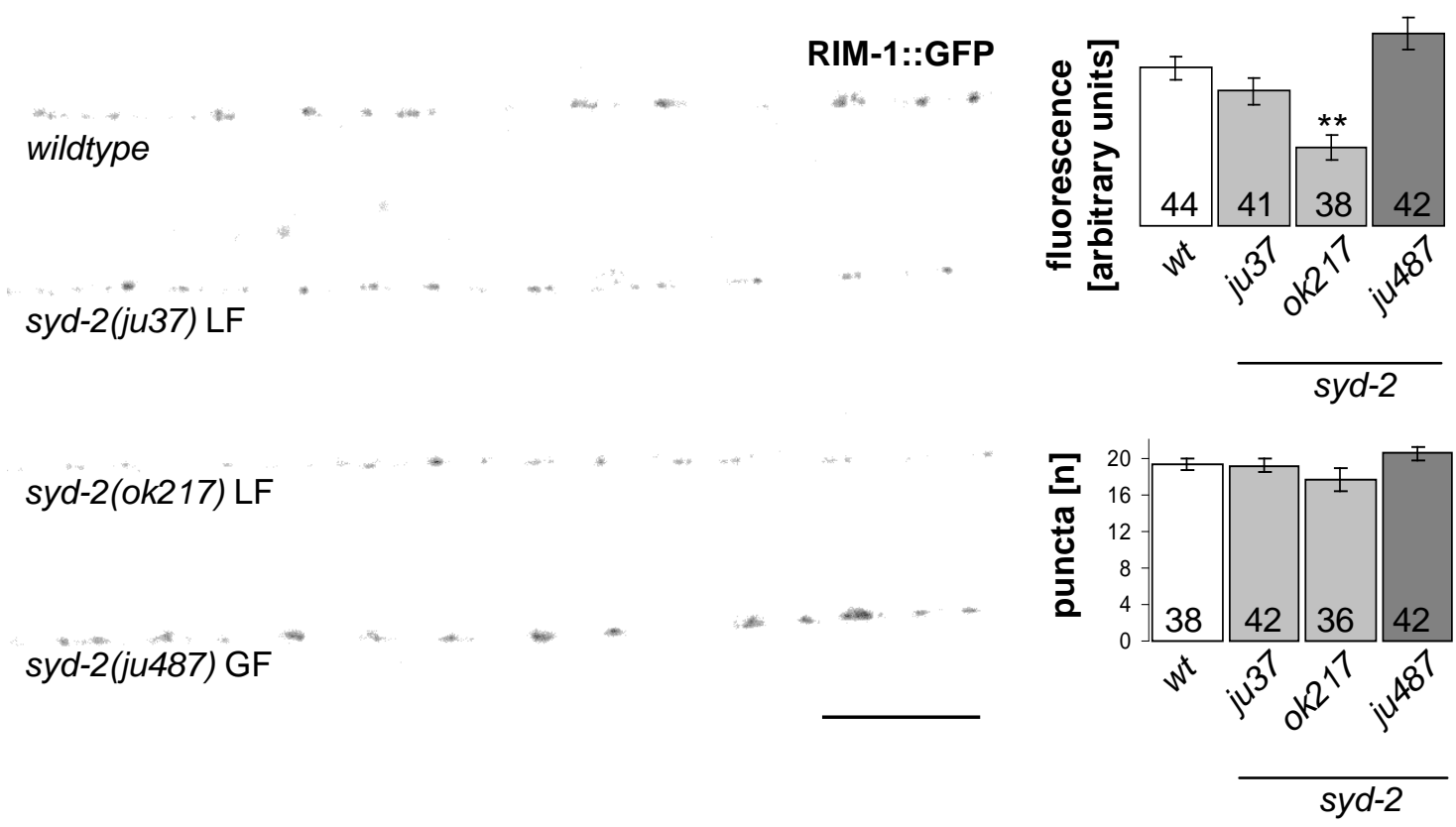

Fig. IV.15 Localization of AZ marker RIM-1/UNC-10 at NMJs is normal in syd-2 mutants. Fluorescence quantification of AZ marker proteins RIM-1::GFP in wild type and syd-2 mutant strains reveals largely normal synaptic localization in cholinergic DA and DB motor neurons. The number of puncta is similar to wild type in all mutants. Overall fluorescence is reduced in syd-2(ok217) LF mutants. Scale bar is $10 \mu \mathrm{m}$. Error bars represent S.E.M. Statistics: ANOVA and Dunnett's post-test. $\left({ }^{*} \leq 0.05 ;{ }^{* *} \leq 0.01 ;{ }^{* * *} \leq 0.001\right)$.

\subsection{Synaptic transmission is affected in syd-2 mutant animals}

To ensure proper signal transduction, SVs have to be efficiently recruited to the $A Z$ to dock and fuse with the presynaptic membrane (Pfeffer, 1999, Stigloher et al., 2011). As recently reported, synaptic transmission and the recruitment and docking of SVs is impaired in syd-2(ju37) LF and unc-10(md1117) LF mutants (Stigloher et al., 
2011). Our analysis of docked vesicles at the $A Z$ membrane in synaptic midsections of both syd-2 LF alleles (ju37 and ok217) could reproduce the reported findings (Fig. IV.16).

The number of docked SVs was comparable to wild type in syd-2 GF mutants. Surprisingly, fewer vesicles were docked in the syd-2 super-GF mutants within $90 \mathrm{~nm}$ (Bin I to III) of the DP (Fig. IV.16). This is similar to the phenotype observed in syd-2 LF mutants.
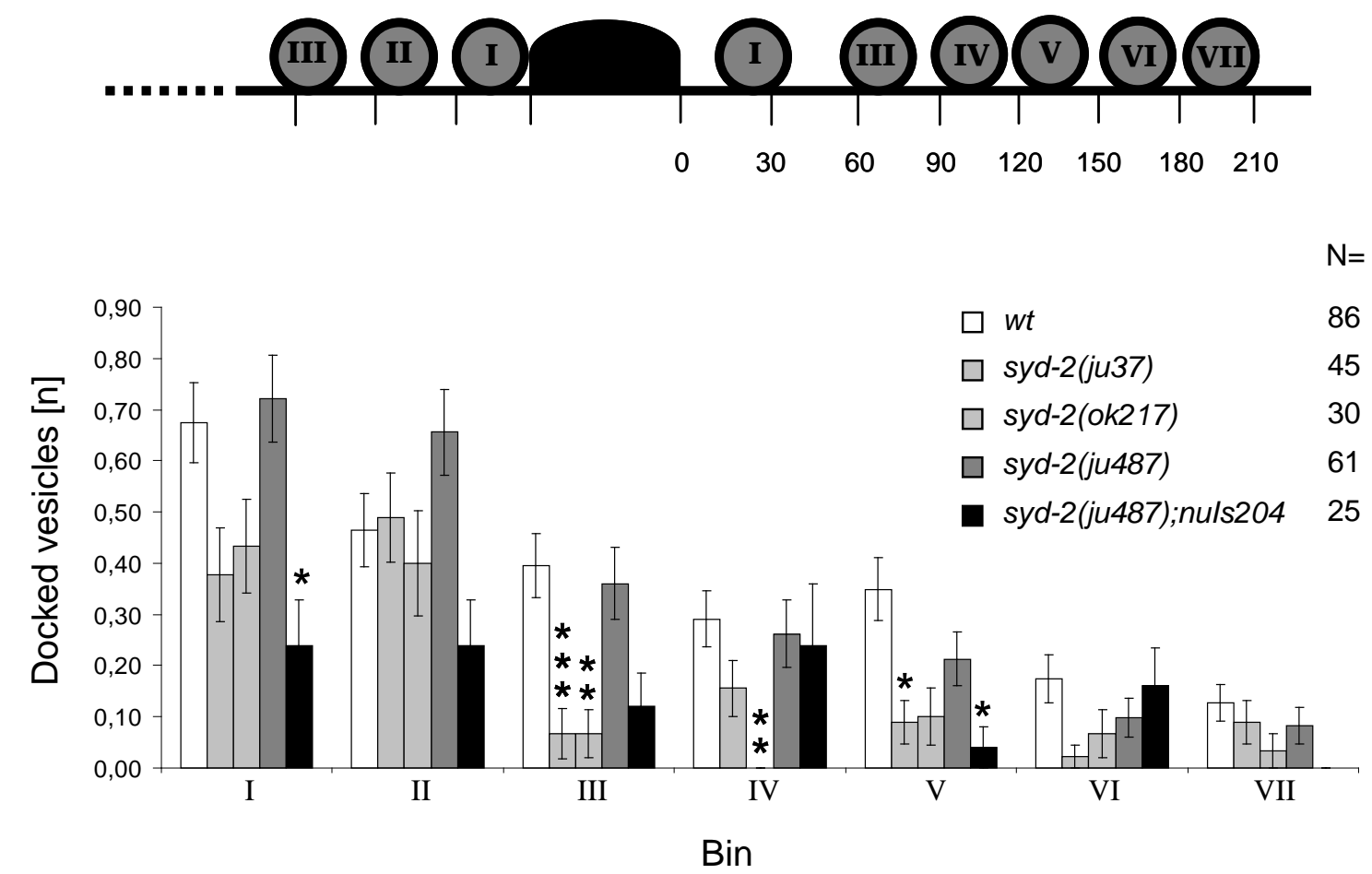

Fig. IV.16 Reduced numbers of SVs are docked to the AZ membrane in syd-2 LF mutants. SVs in direct contact with the presynaptic membrane of the $A Z$ were assigned to bins according to their distance from the DP as indicated on top. The average number of docked SVs per profile is reduced in syd-2 LF mutant alleles ju37 and ok217 as well as syd-2 super-GF (ju487; nuls204) mutants. Syd2(ju487) GF mutants show wild type-like numbers of docked SVs. Error bars represent S.E.M. Statistics: Kruska-Wallis test and Dunn's multiple comparison test. $\left({ }^{*} \leq 0.05 ;{ }^{* *} \leq 0.01 ;{ }^{* * *} \leq 0.001\right)$. Sample preparation, imaging and quantification were done by Dr. Jan Hegermann and the author.

As expected from the reduced number of docked SVs available for fusion, syd-2 LF and syd-2 super-GF show a reduced evoked response (Fig. IV.17). The results indicate that SYD-2 plays a pivotal role in assembling a functional transmission machinery that effectively delegates SVs to the $A Z$ for triggered release. Although the SYD-2 GF protein has a positive effect on DP formation by increasing its size and possibly the number of potential fusion sites, it does not notably enhance synaptic 
transmission. The overexpression of SYD-2 R184C GF protein in the syd-2 super-GF mutant does not improve DP formation and synaptic transmission, but has a rather negative effect, supporting the notion that other limiting factors might constrain the effect of SYD-2 super-GF.

\section{Evoked response $5 \mathrm{mM} \mathrm{Ca}$}

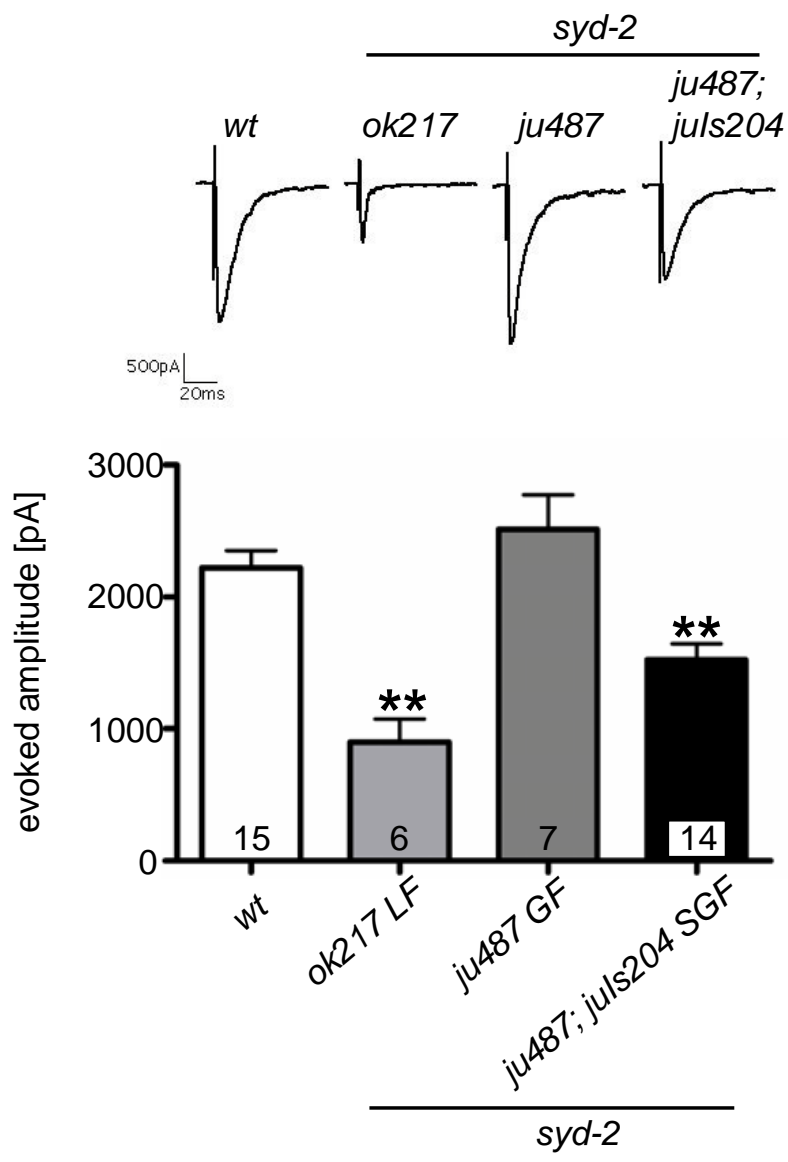

Fig. IV.17 Evoked response is reduced in syd-2 LF and syd-2 super-GF mutants. Representative traces of evoked synaptic currents recorded from body wall muscles (BWMs) via whole-cell voltage-clamp of wild type and syd-2 mutants are shown. Quantification shows that the amplitude of the evoked response is significantly reduced in syd-2(ok217) LF and syd-2(ju487); juls204 super-GF mutants. Evoked response in syd2(ju487) GF mutants is normal. Error bars represent S.E.M. Statistics: ANOVA and Dunnett's post-test. (* $\leq 0.05 ;{ }^{* *} \leq 0.01 ;{ }^{* * *} \leq 0.001$ ). Recordings were done by Prof. Janet Richmond.

The specialized HSNs release serotonin and regulate egg-laying together with the cholinergic VC4 and VC5 neurons by innervating vm2 vulva muscles (White et al., 1986, Shen et al., 2004). Similar proteins are involved in synapse assembly in NMJ and HSN synapses (Patel et al., 2006). Egg-laying behavior therefore provides an easy read-out for impaired synaptic transmission. Sluggish locomotion and defective egg-laying has been reported previously for syd-2(ju37) LF mutants (Zhen and Jin, 1999). Syd-2(ok217) LF mutants displayed a similar egg-laying phenotype in which the initiation of egg-laying is delayed for about 6h compared to wild type animals and eggs are retained longer in the gonad (data not shown). Consequently, fewer eggs are laid by syd-2 LF mutants (Fig. IV.18). 


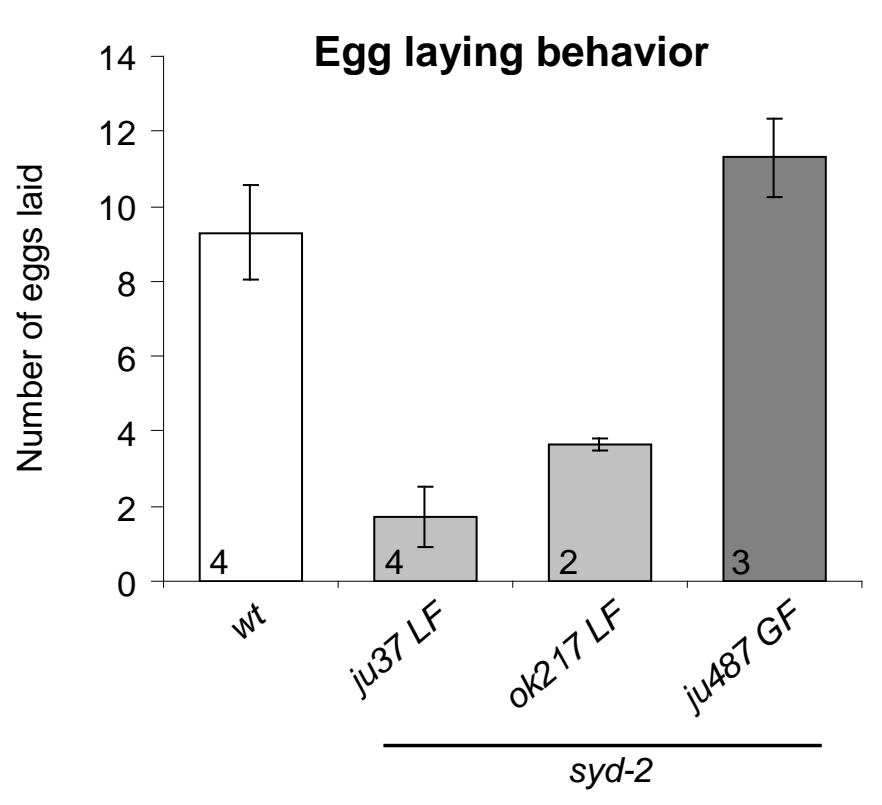

Fig. IV.18 syd-2 LF mutants are egglaying defective. Number of eggs laid by 10 worms after $2 \mathrm{~h}$ was quantified and averaged over three trials. Loss of SYD-2 results in impaired synaptic signaling of motor neurons VC4 and VC5 and hermaphrodite specific neurons onto vulva muscles. Number of eggs laid by syd-2 LF mutants is strongly reduced. Egg-laying in syd-2 GF mutants is comparable to wild type. Error bars represent S.E.M.

To test whether this effect is only attributed to the presynaptic defects described above or is also due to postsynaptic alterations, we carried out levamisole and aldicarb assays. Aldicarb is an acetylcholine (ACh) esterase inhibitor that prevents the degradation and thus removal of $\mathrm{ACh}$ from the synaptic cleft. Subsequent continuous stimulation of the postsynaptic ACh receptors in the muscle cells leads to paralysis of the animal.

In agreement with reported transmission defects, syd-2(ju37) and syd-2(wy5) LF mutants have been shown to be moderately resistant to aldicarb (Zhen and Jin, 1999, Klassen et al., 2010). My aldicarb assays could reproduce this finding. Moderate aldicarb resistance phenotypes were observed for syd-2 LF mutant alleles ju37 and ok217 (Fig. IV.19), using unc-10(md1117) mutants a reference strain for a strong aldicarb resistant phenotype (Koushika et al., 2001, Deken et al., 2005). I also tested syd-2(ju487) GF and syd-2(ju487); juls204 super-GF mutants. In syd-2 GF mutants the evoked response was unaltered (Fig. IV.17), yet they were hypersensitive compared to wild type and tom-1(ok285), a marker for strong aldicarb hypersensitivity (Dybbs et al., 2005). The total number of docked vesicles per synapse was slightly increased in GF mutants compared to wild type (data not shown). More fusion sites for SVs in elongated DPs may not alter synaptic transmission drastically but could account for aldicarb hypersensitivity.

Even more surprising, syd-2 super-GF mutants with defective SV docking and clearly reduced evoked response are rather sensitive to aldicarb. So far, I am not able to 
conclusively explain this result. Possibly enhanced activation via regulating command interneurons in indissected animals leads to a stronger release of $\mathrm{ACh}$ in these mutants (de Bono and Maricq, 2005).

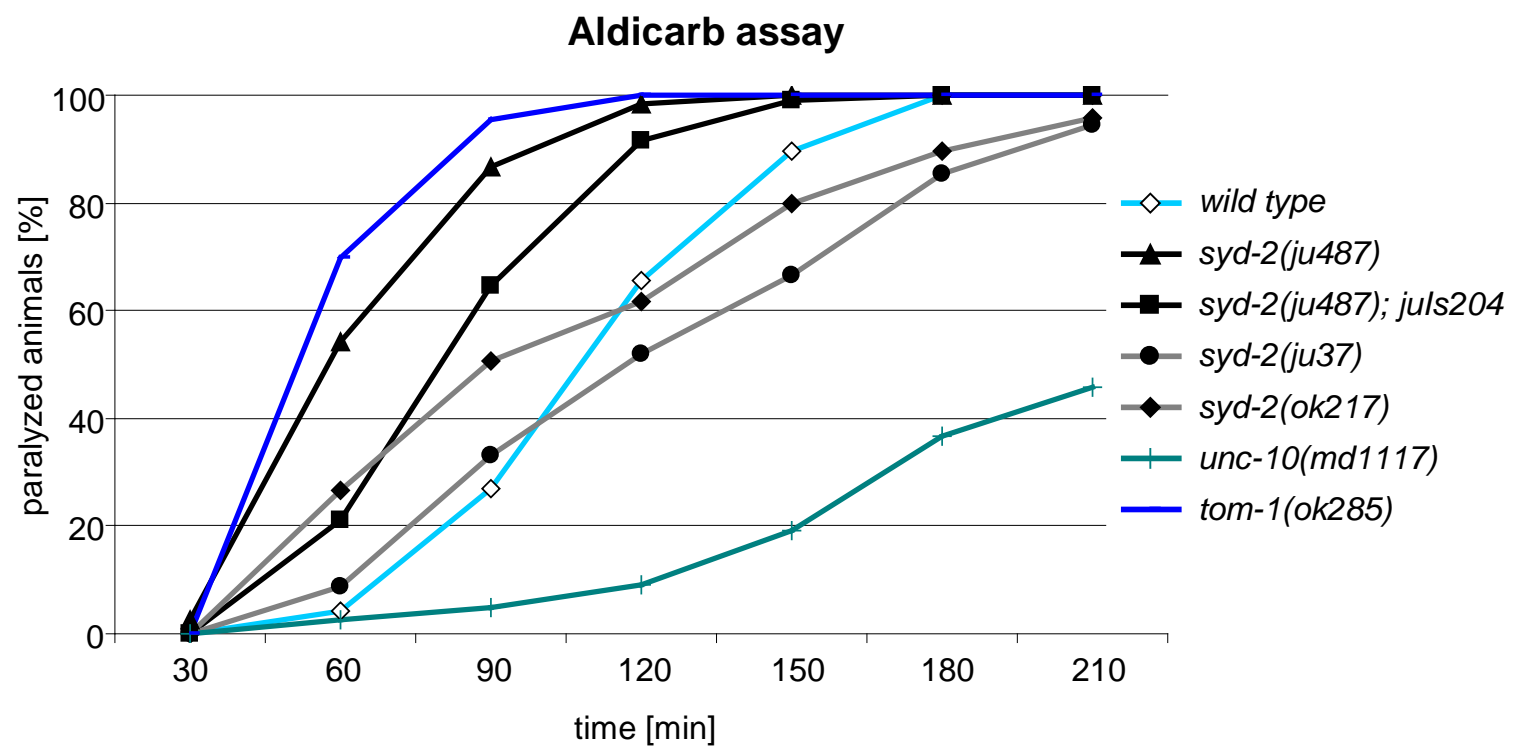

Fig. IV.19 Aldicarb sensitivity is altered in syd-2 mutants. Both syd-2 LF mutants show moderate resistance to aldicarb whereas syd-2 GF and super-GF mutants were hypersensitive compared to wild type worms. 30 worms were scored for paralysis every 30 minutes on $1.5 \mathrm{mM}$ aldicarb plates. tom-1 and unc-10 strains were used as markers for strong aldicarb sensitivity and resistance, respectively, as suggested by (Mahoney et al., 2006). Four trials were averaged for each time point.

To test for defects at the postsynaptic site, we applied levamisole, an agonist for levamisole-sensitive ACh receptors. Continuous activation of these receptors in muscle cells by levamisole eventually leads to paralysis. Mutants with defective clustering of levamisole receptors exhibit resistance due to reduced signal transduction. All syd-2 mutants tested except for syd-2(ok217) LF showed wild typelike sensitivity to levamisole, suggesting normal postsynaptic levamisole-sensitive ACh receptor clustering (Fig. IV.20). 


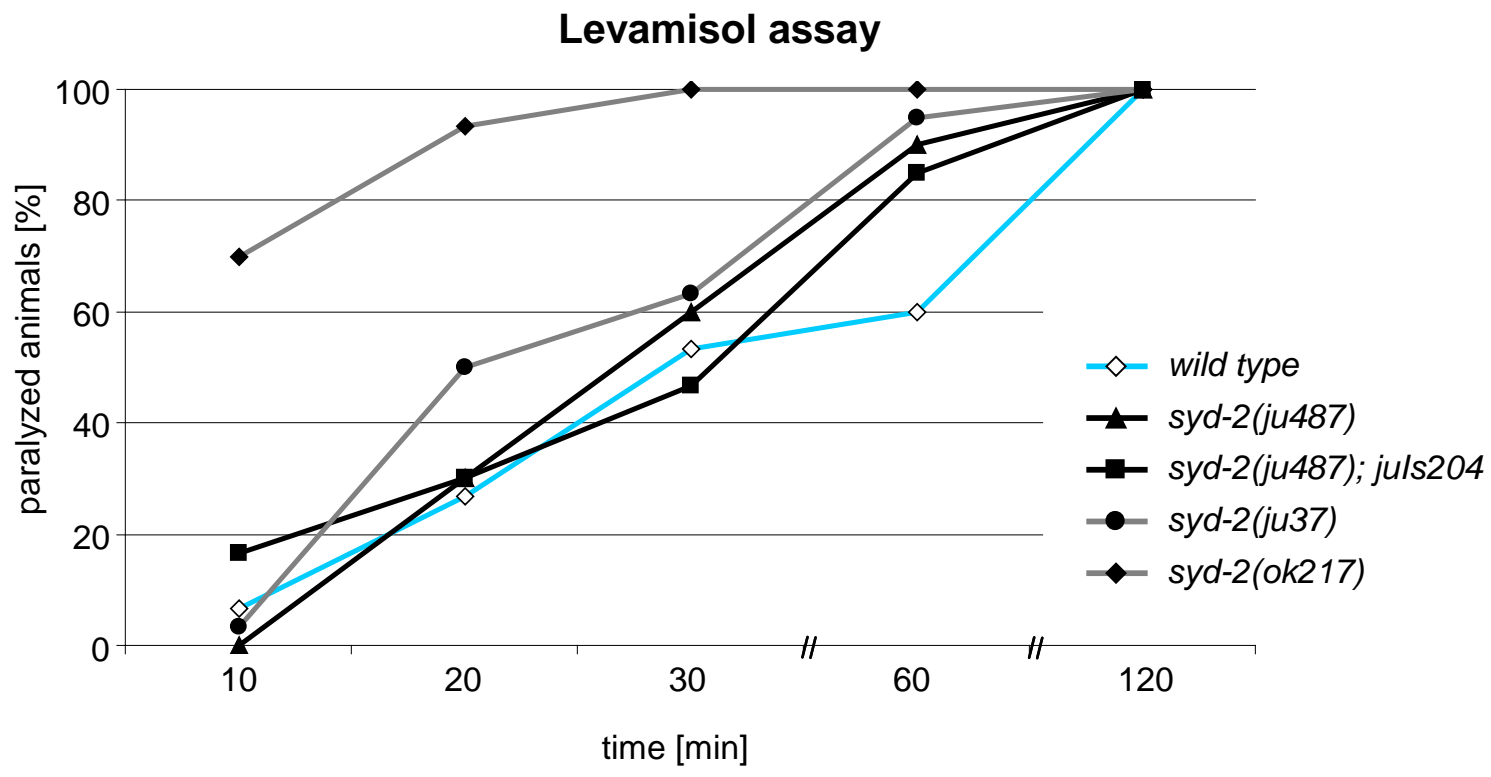

Fig. IV.20 Levamisole sensitivity is normal in syd-2 mutants except for syd-2(ok217) LF. Syd2(ju37) LF, syd-2ju487) GF and syd-2(ju487); juls204 super-GF mutants show similar levamisole sensitivity as wild type worms. The syd-2(ok217) LF mutant is more sensitive to levamisole. 10 worms per plate were scored for paralysis every 10 minutes on $0.2 \mathrm{mM}$ levamisole plates. Three trials were averaged for each time point.

Vertebrate Liprin- $\alpha$ has been implicated not only in pre- but also postsynaptic function by indirectly targeting AMPA ( $\alpha$-amino-3-hydroxy-5-methylisoxazole-4-propionic acid) receptors and cadherin- $\beta$-catenin complexes (reviewed in Spangler and Hoogenraad, 2007). The deletion in the syd-2(ok217) allele disturbs most of the N-terminal coiled coil domains known to be important for interaction with various other synaptic proteins (Ko et al., 2003b, Spangler and Hoogenraad, 2007). It is possible that binding domains involved in postsynaptic function of SYD-2 are lost in the ok217 allele but not in ju37, leading to postsynaptic defects. Although the ok217 allele has been crossed out several times, additional background mutations could exist and influence the phenotype.

\subsection{The ability of SYD-2 to promote the formation of longer DPS depends on ELKS-1}

As pointed out in the introduction, SYD-2 has been shown to interact with a number of synaptic proteins. Thus, it is likely that one or more interaction partners augment 
its function (alo refer to 2.2.5). ELKS-1 is the C. elegans homolog of vertebrate ELKS (glutamine, leucine, lysine, and serine-rich), a protein mainly composed of coiled coil domains (Fig. IV.21) (Ohtsuka et al., 2002). Interactions with other AZ proteins like RIM1, Munc13-1 (Ohtsuka et al., 2002) and also Liprin- $\alpha$ (Ko et al., 2003b) have been reported in vertebrates, Drosophila and C. elegans.

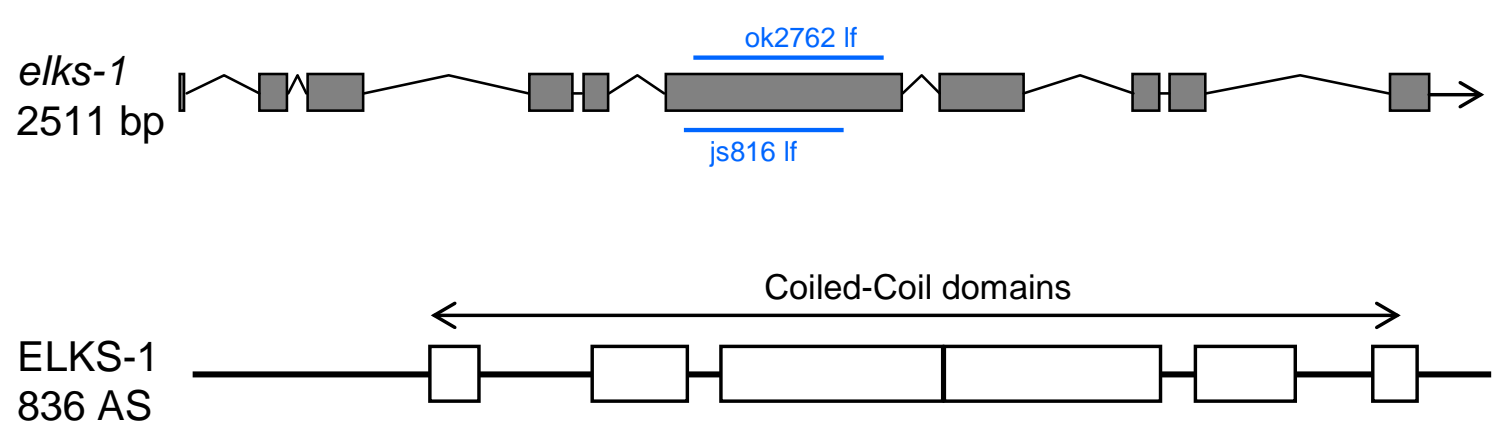

Fig. IV.21 elks-1 gene and ELKS-1 protein structure. Loss of function mutations (blue) are indicated in the gene model. ELKS-1protein mainly consists of coiled coil domains. Structure prediction (HUSAR sequence analysis package) was adapted from (Wagh et al., 2006).

Although Drosophila mutants lacking the ELKS homolog Bruchpilot have clear synaptic defects (Kittel et al., 2006, Wagh et al., 2006), elks-1 LF mutants in C. elegans do not show any obvious phenotype in synaptic transmission or $A Z$ morphology (Deken et al., 2005). However, no detailed EM analysis of AZ ultrastructure and no extensive analysis of elks mutants in other organisms has been performed. Nevertheless, ELKS-1 seems to play an important regulatory role in $C$. elegans HSN assembly. SYD-2 GF is only able to bypass the requirement of SYD-1, an upstream regulator of AZ assembly (Hallam et al., 2002, Patel et al., 2006), in the presence of ELKS-1 (Dai et al., 2006).

Yet, the kind of interaction or dependency between SYD-2 and ELKS-1 remains unclear. To gain more insight into the function of ELKS-1 in synapse assembly, I initially performed ultrastructural analysis of two elks-1 null mutants, which exhibit a deletion in exon 6 (Fig. IV.21) (Deken et al., 2005). Neither elks-1 mutant revealed obvious alterations in NMJ AZ or motor neuron axon morphology (Fig. IV.22; data for elks-1(js816) not shown). 


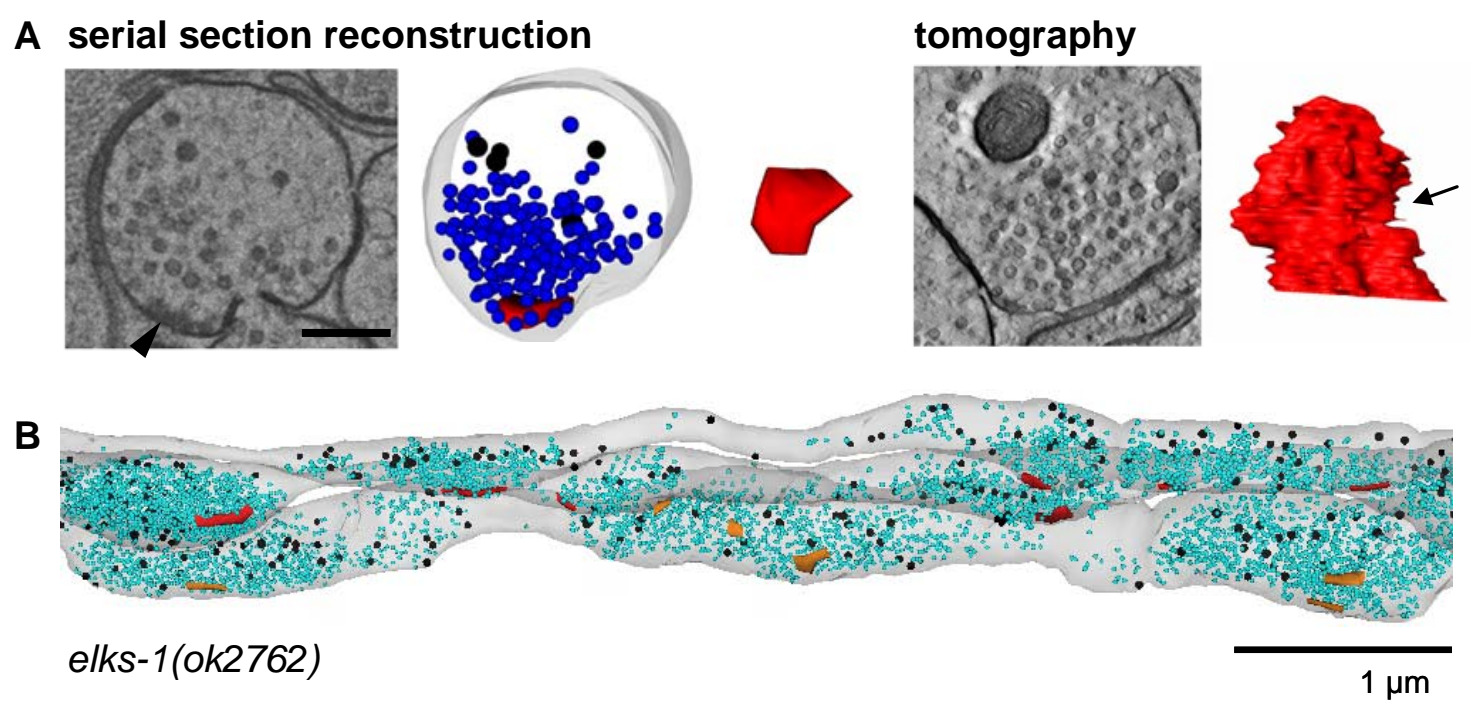

Fig. IV.22 Axon and synapse morphology is unaltered in elks-1 LF mutants. (A) Serial section reconstructions to visualize DPs size and vesicle clusters as well as tomographic reconstructions of the NMJ DP were performed in elks-1 mutants (compare to wild type in Fig. IV.10). Scale bar is 200 $\mathrm{nm}$. 3D reconstructions did not reveal any alterations in synapse morphology in terms of size, vesicle clustering and bay-structure (arrow) in elks-1(ok2762) mutants. (B) Serial section reconstruction of cholinergic and GABAergic motor neurons in the ventral nerve cord shows normal axon morphology as well as SV and DCVs localization (compare to wild type in Fig. IV.11).

I analyzed the total number of SVs and DCVs per AZ as well as the number of docked vesicles at the $A Z$ membrane per profile as described above for syd-2 mutants SV and DCV transport to synaptic sites and docking to the presynaptic membrane was unchanged in elks-1 LF mutants according to quantifications from electron micrographs (Fig. IV.23). 
A
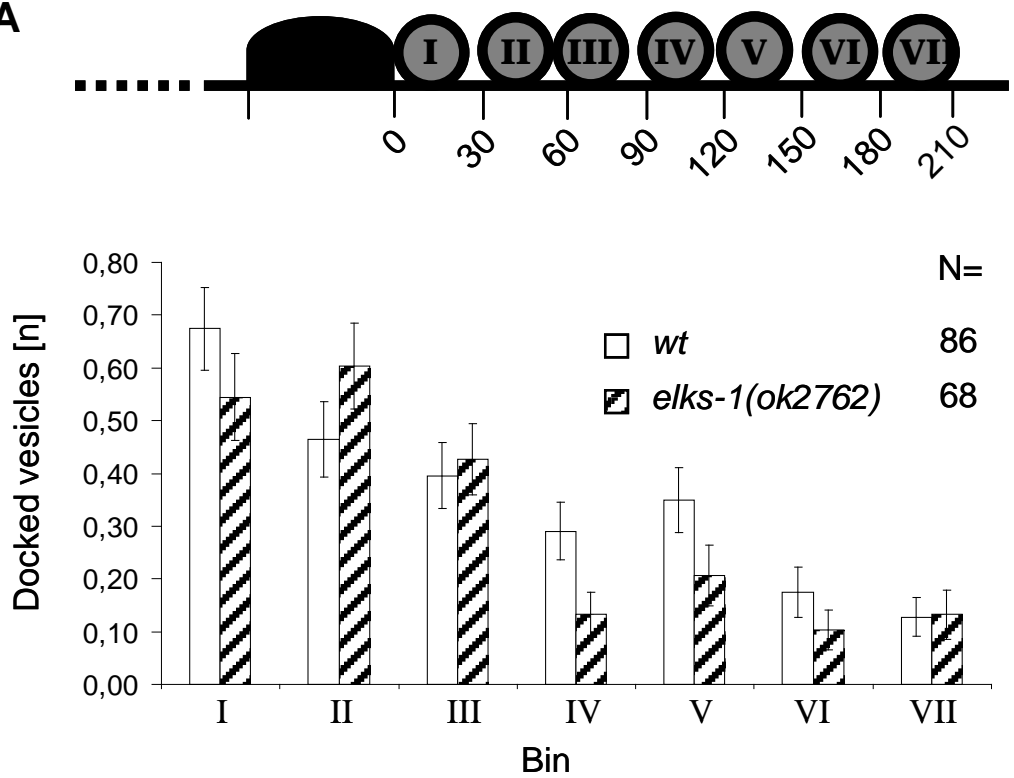

B SVs per synapse

DCVs per synapse
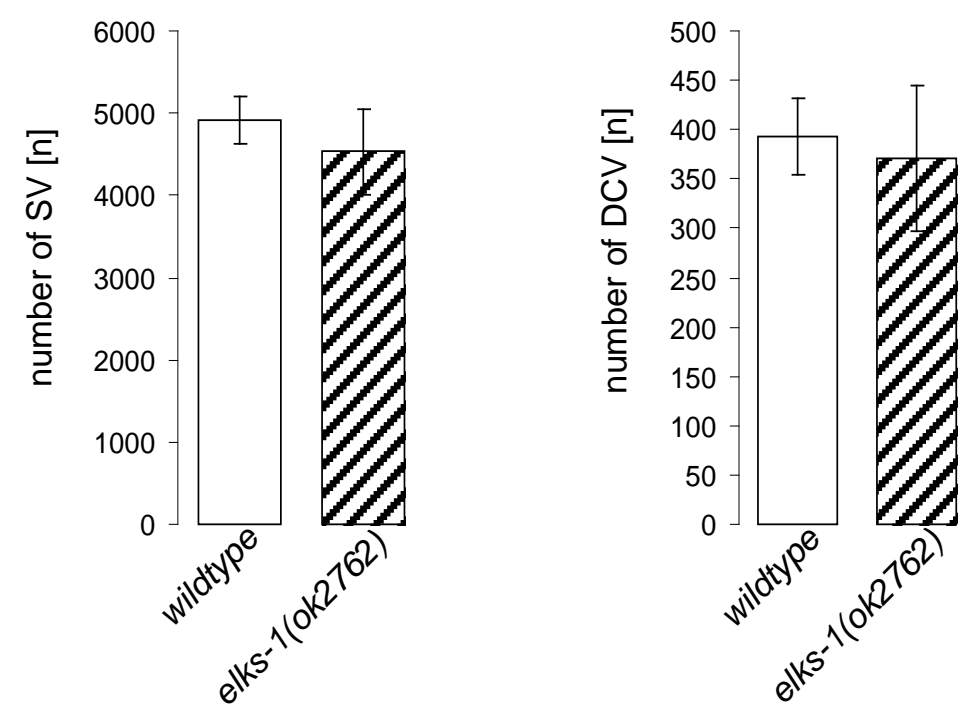

Fig. IV.23 Normal SV docking and vesicle clustering in elks-1 LF mutants. (A): Assignment of SVs directly contacting the presynaptic membrane of the AZ into categories (top) was done as described in Fig. IV.16. The average number of docked vesicles at the AZ membrane per synapse profile is similar to wild type in elks-1 mutants. Error bars represent S.E.M. Statistics: Mann Whitney test. (B): The number of SVs and DCVs in wild type and elks-1 mutants was counted in sections with the DP visible and was normalized to the size of the synaptic terminal. The total number of SVs and DCVs is unaltered in elks-1 mutants. Error bars represent S.E.M. Statistics: Unpaired t test $\left({ }^{*} \leq 0.05\right.$; $\left.{ }^{* *} \leq 0.01 ;{ }^{* * *} \leq 0.001\right)$. 
In agreement with these observations and published data (Deken et al., 2005), no alteration in evoked response amplitude could be detected in elks-1 LF mutants (Fig. IV.24).

\section{Evoked response $5 \mathrm{mM} \mathrm{Ca}$}

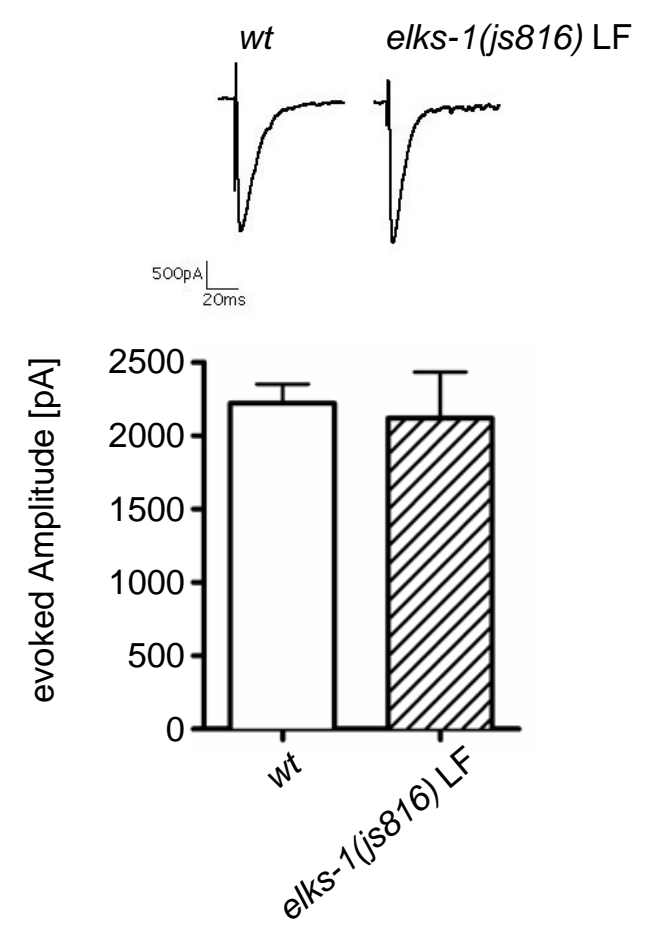

Fig. IV.24 Normal evoked response in elks-1 LF mutants. Representative traces of evoked synaptic currents recorded from BWM via whole-cell voltageclamp in wild type and elks-1 mutants are shown. Quantification shows that the amplitude of the evoked response in elks-1(js816) LF mutants is comparable to wild type. Error bars represent S.E.M. Statistics: Unpaired $t$ tes $\left.\leqslant 0.05 ; \quad * * \leq 0.01 ; \quad{ }^{* * *} \leq 0.001\right)$. Recordings were done by Prof. Janet Richmond.

Co-immuno-precipitation has previously been utilized to show that interaction between ELKS-1 and SYD-2 GF containing the point mutation R184C is enhanced compared to ELKS-1 interaction with wild type SYD-2 (Dai et al., 2006). The GF mutation is located in the $\mathrm{N}$-terminal coiled coil domains within the highly conserved LH1 domain of SYD-2. To investigate more precisely which region specifically facilitates the binding, the interaction between ELKS-1 and the highly conserved LH domains of SYD-2 (Taru and Jin, 2011) was examined by in-vitro pull-down assay and yeast two hybrid studies.

SYD-2N (aa 1-517) or SYD-2N R184C (aa 1-517), which included both LH domains, and ELKS-1 were tagged to either His6 or GST, respectively. Western blot analysis of the eluted protein complexes clearly showed greater amounts of ELKS-1 pulled down with SYD-2N R184C than with wild type SYD-2 N (Fig. IV.25). 
A

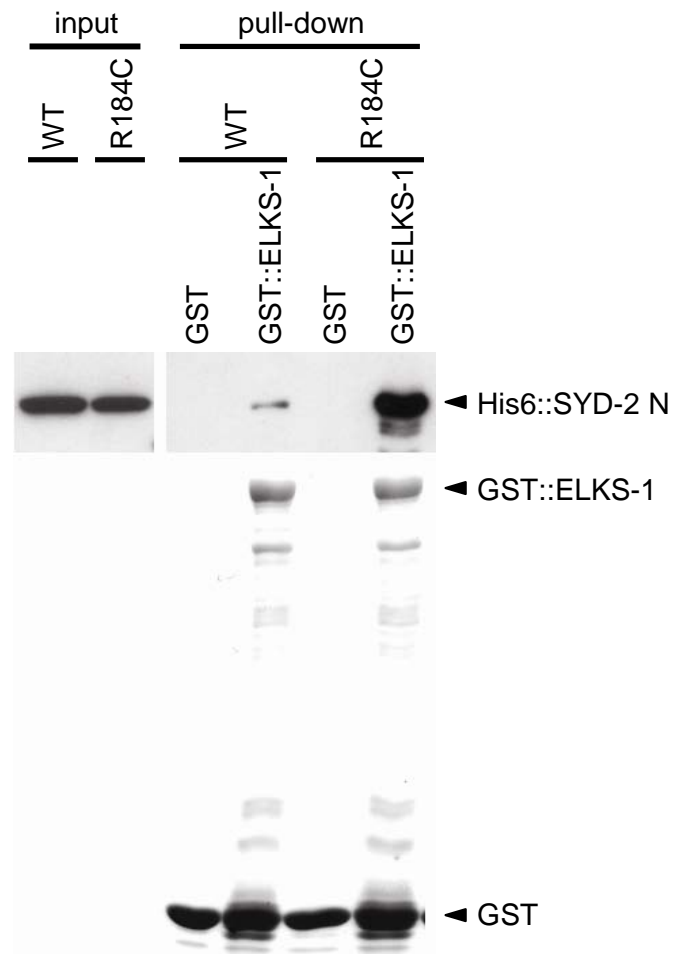

B

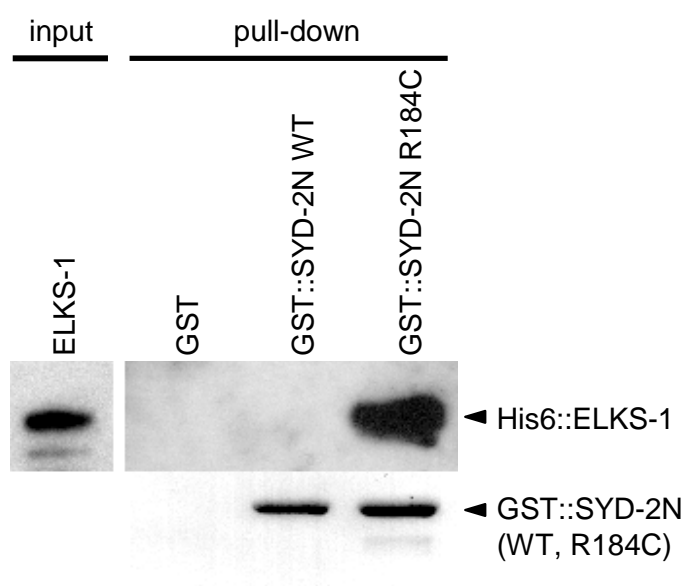

Fig. IV.25 The amino acid exchange R184C in SYD-2 GF protein leads to enhanced interaction with ELKS-1. Interaction between ELKS-1 and the N-terminus of wild type (WT) SYD-2 or SYD-2 R184C, respectively, was examined. Interaction of (A) His6::SYD-2 N and GST::ELKS-1 or (B) His6::ELKS-1 and GST::SYD-2 $\mathrm{N}$ was shown by in vitro pull-down assay. Proteins levels were analyzed by Western blotting with anti-His6 antibody (upper panels) and by Ponceau S stain (lower panels). More SYD-2 N (aa 1-517) R184C compared to wild type SYD-2 is pulled down with ELKS (A). Vice versa more ELKS-1 binds to SYD-2 N (aa 1-517) R184C than to wild type SYD-2 (B). Experiments were carried out by Prof. Hidenori Taru.

To further narrow down the SYD-2 binding site for ELKS-1, I performed yeast-twohybrid interaction assays with full length ELKS-1 and the LH1 or R184C LH1 domain (aa 91-207) of SYD-2, respectively. On histidine auxotrophy plates, the SYD-2 GF substitution R184C in the LH1 domain clearly led to a strong interaction with ELKS, while interaction with wild type LH1 was too weak or transient to be detected (Fig. IV.26). 


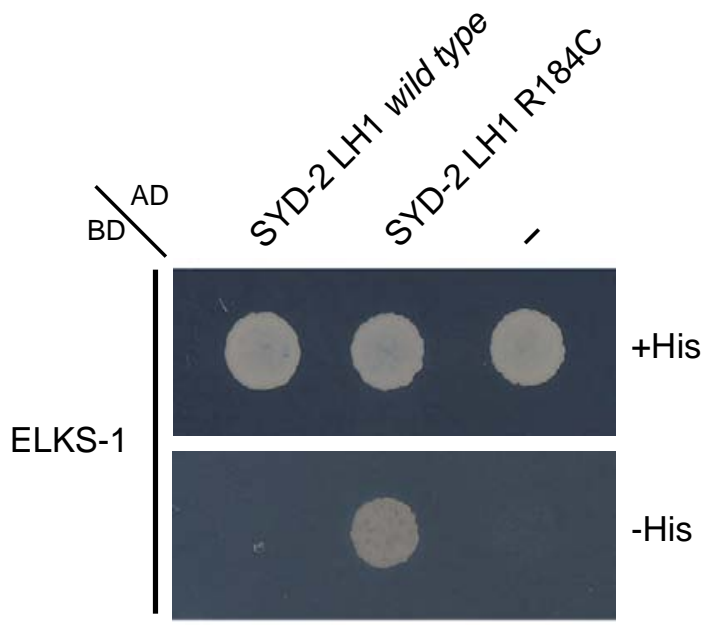

Fig. IV.26 SYD-2 LH1 with R184C amino acid exchange shows enhanced binding to ELKS-1. Yeast two hybrid analysis of SYD-2 LH1 or SYD-2 LH1 R184C and full length ELKS-1, respectively. HIS growth assays indicates binding of ELKS-1 to SYD-2 LH1 R184C. Interaction with wild type SYD$2 \mathrm{LH} 1$ was too weak to be detected.

Both assays revealed that enhanced interaction of ELKS-1 with GF SYD-2 is mediated specifically by the LH1 domain. Taru and Jin have recently assigned the ability of homomeric dimer formation to the LH1 domain of SYD-2 using gel-filtration and multiangle light scattering (MALS) experiments (Taru and Jin, 2011). Similar experiments were conducted for this study with the R184C SYD-2 N-terminus (aa 91207). Enhanced dimerization and even oligomerization were detected for the R184C LH1 domain (Fig. IV.27, blue trace). As reported, wild type LH1 also forms dimers, but predominantly exists as monomer (Fig. IV.27, red trace) (Taru and Jin, 2011).

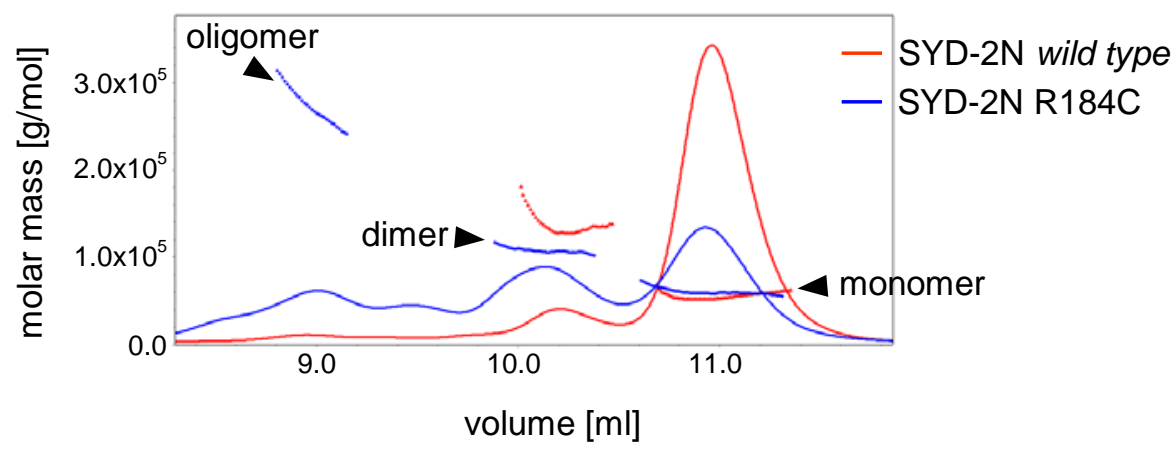

Fig. IV.27 The highly conserved LH1 domain in the SYD-2 N-terminus oligomerizes more easily when the R184C substitution is present. SYD-2 LH1 (aa 91-207) fused to maltose binding protein was analyzed by gel-filtration with size-exclusion column and multiangle light scattering (MALS). SYD- 
$2 \mathrm{LH} 1$ wild type protein is mainly detected as monomers at low concentration (red line) whereas SYD2 LH1 R184C preferentially forms dimers or higher oligomers (blue line). The chromatogram indicates the differential reflective index and molecular mass $(\mathrm{g} / \mathrm{mol})$ for each peak calculated from light scattering data. The experiments were conducted by Prof. Hidenori Taru.

The requirement of ELKS-1 for SYD-2 GF to promote AZ assembly in a SYD-1independent manner has been shown via fluorescence microscopy and biochemical assays (Dai et al., 2006). However, the effect on synaptic ultrastructure has not been investigated so far. We generated syd-2; elks-1 double mutants with the LF and GF alleles of syd-2 and compared them to the respective single mutants.

As shown above, elks-1 alone had no effect on DP ultrastructure (Fig. IV.22). Double LF mutants syd-2(ju37); elks-1 exhibited the same phenotype as single syd-2(ju37) mutants in terms of DP length, suggesting that both proteins function in the same pathway (Fig. IV.28).

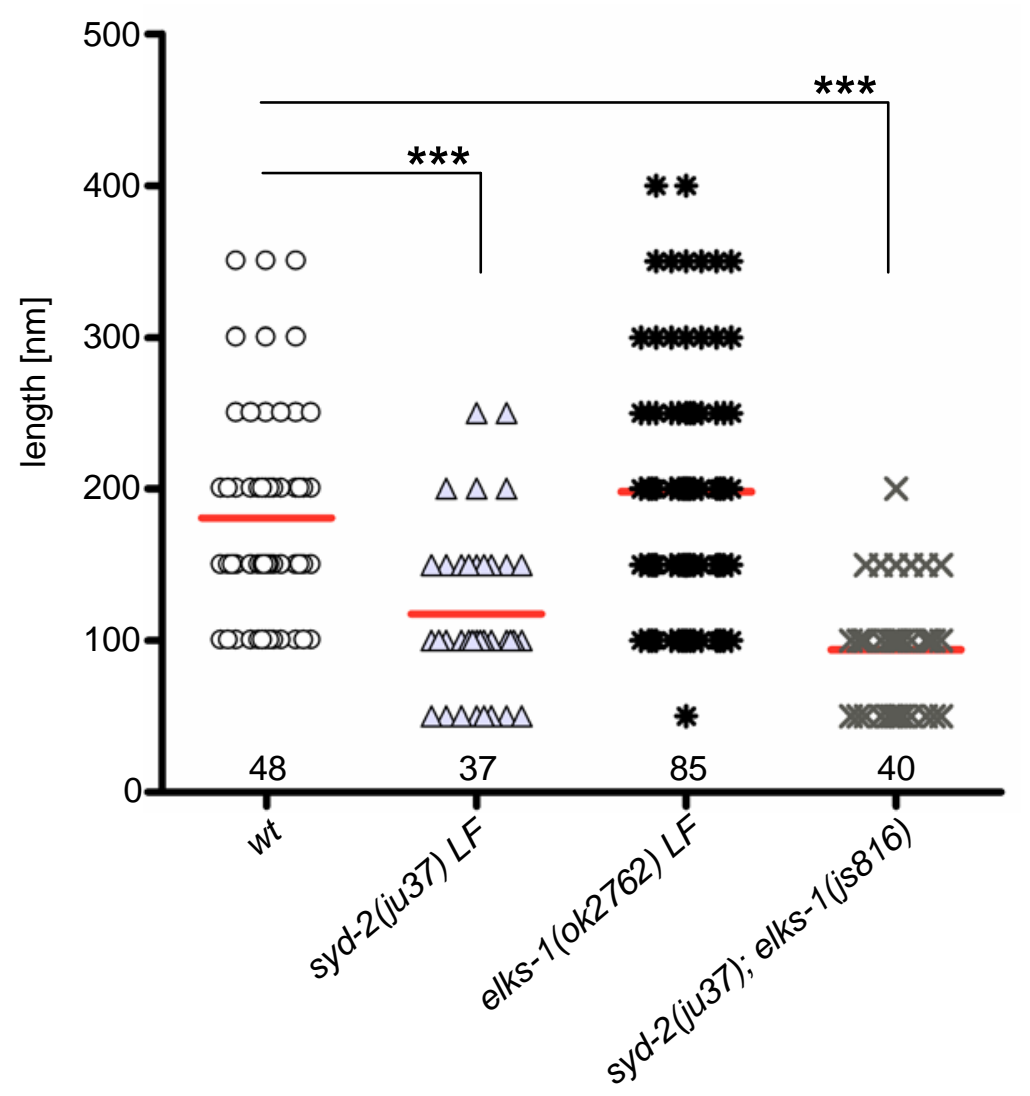

Fig. IV.28 Double syd-2; elks-1 LF mutants are similar to syd-2 single LF mutants. DP length along the axons was analyzed from $50 \mathrm{~nm}$ serial cross sections. Single syd-2(ju37) LF mutants show reduced DP size while elks-1(ok2762) LF mutants show no obvious phenotype. ELKS-1 is known to be required for syd-2 GF functionality (Dai et al., 2006), but loss of ELKS-1 in the syd-2 LF background 
does not enhance the phenotype of the single syd-2 LF mutants. The mean is indicated by the red line. $\mathrm{N}$ is indicated below data points. Statistics: Kruska-Wallis test and Dunn's multiple comparison test. $\left({ }^{*} \leq 0.05 ;{ }^{* *} \leq 0.01 ;{ }^{* * *} \leq 0.001\right)$.

In line with these findings, loss of ELKS-1 in the syd-2 GF background clearly inhibits the formation of elongated DPs observed in the syd-2 GF single mutant (Fig. IV.29), supporting the proposed requirement of ELKS-1 for SYD-2 GF to be functional (Dai et al., 2006).

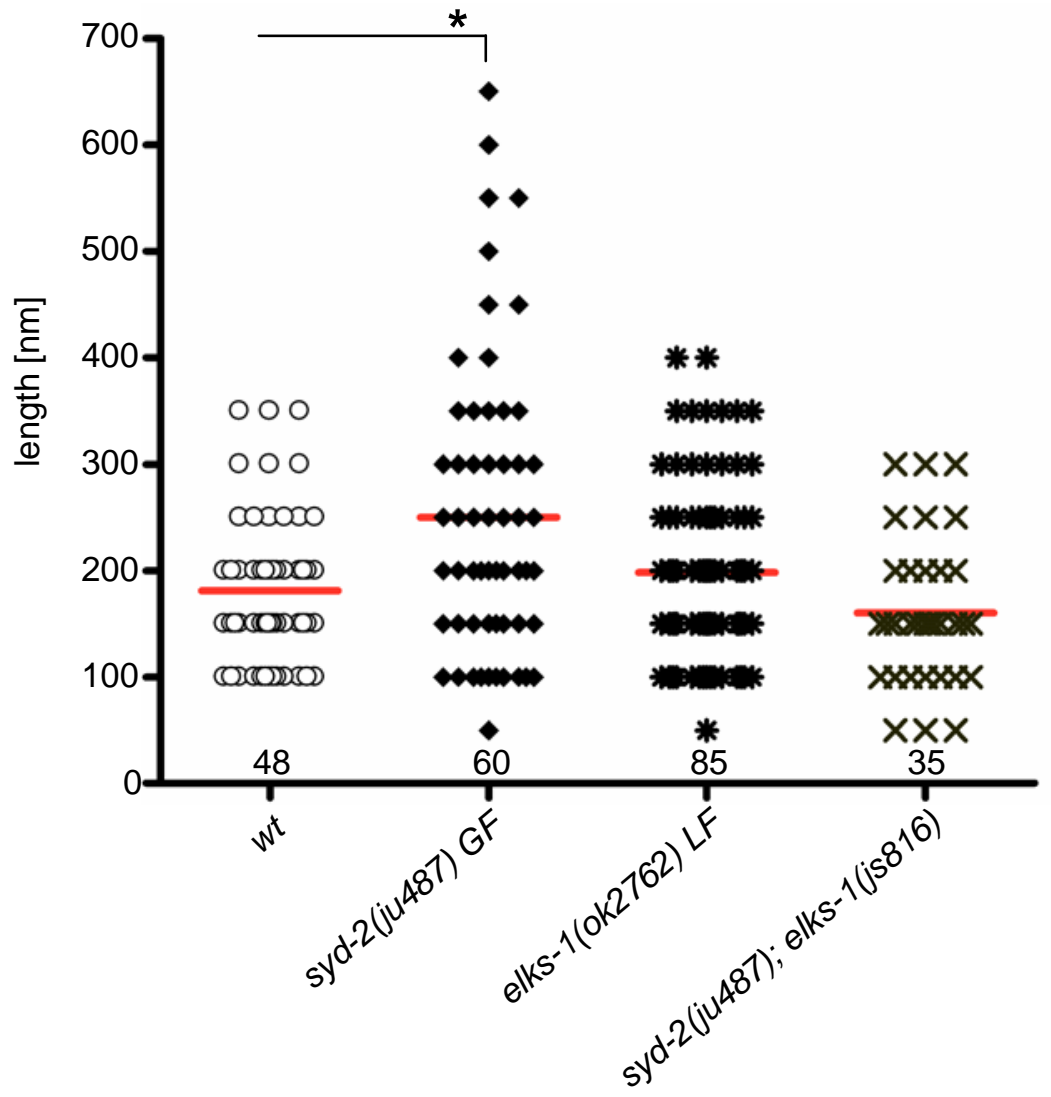

Fig. IV.29 ELKS-1 is required in the syd-2 GF mutant to form elongated NMJ DPs. DP length along the axons was analyzed from $50 \mathrm{~nm}$ serial cross sections. Single syd-2 GF mutants can form strongly elongated DPs that are not found wild type animals. These longer DPs are lost in syd-2 GF; elks-1 LF double mutants while wild type-size DPs are still formed. The mean is indicated by the red line. $\mathrm{N}$ is indicated below data points. Statistics: Kruska-Wallis test and Dunn's multiple comparison test. $\left({ }^{*} \leq 0.05 ;{ }^{* *} \leq 0.01 ;{ }^{* * *} \leq 0.001\right)$.

To test whether the loss of elongated DPs in syd-2 GF; elks-1 LF double mutants has an impact on synaptic transmission, electrophysiological recordings were performed. 
No significant difference in evoked response amplitude could be observed at either low or high extracellular calcium (Fig. IV.30; $0.5 \mathrm{mM}$ calcium data not shown). As wild type size DPs are still formed in the syd-2(ju487);elks-1(js816) mutants, this finding was not unexpected.

\section{Evoked response $5 \mathrm{mM} \mathrm{Ca}$}

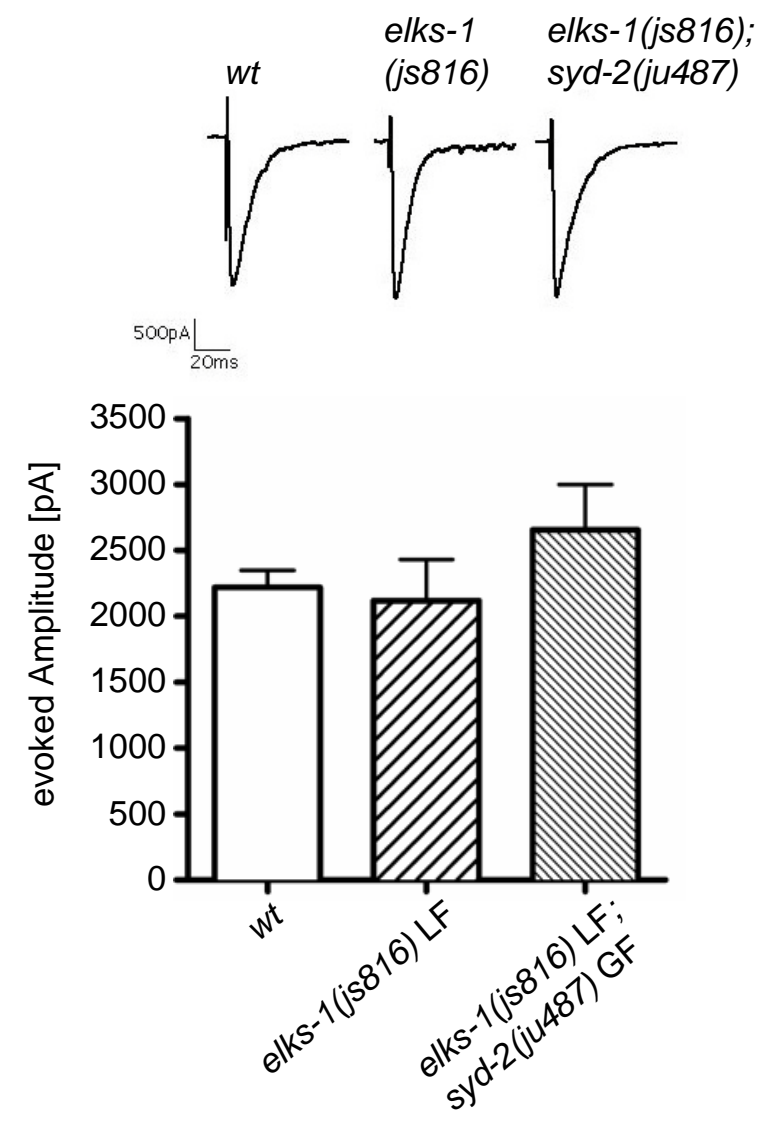

Fig. IV.30 Evoked response is not altered in elks-1(js816); syd-2(ju487) double mutants. Representative traces of evoked synaptic currents recorded from BWM via whole-cell voltage-clamp in wild type, elks1(js816) and elks-1(js816); syd-2(ju487) double mutants are shown. Quantification shows that loss of elks-1 in the syd-2 GF background does not affect the amplitude of the evoked response in these mutants. Error bars represent S.E.M. Statistics: ANOVA and Dunnett's post-test $\leq Q .05 ; \quad{ }^{* *} \leq 0.01$; $\star * \star \leq 0.001)$. Recordings were done by Prof. Janet Richmond.

On the basis of the presented ultrastructural and biochemical evidence, I propose ELKS-1 to function in DP assembly through the interaction with SYD-2.

\subsection{Loss of the negative regulator RSY-1 enhances elongation of DPs in syd-2 GF mutants}

Localization and size of the AZ DPs must be tightly regulated to ensure controlled synaptic transmission at correct sites. Many proteins promoting AZ assembly have been identified, yet only few counteracting negative regulators have been proposed (van Roessel et al., 2004, Nakata et al., 2005, Ding et al., 2007, Klassen and Shen, 


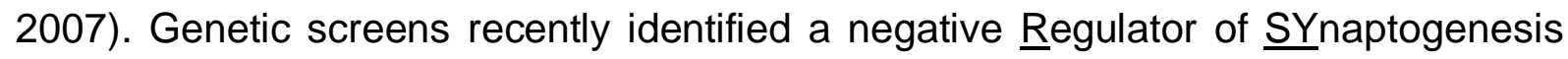
(RSY-1) in HSN neurons directly acting on SYD-2.

RSY-1 compromises the binding of SYD-1 and ELKS-1, which normally promotes SYD-2 function. RSY-1 also directly impairs interaction between SYD-2 R184C and ELKS-1 (Patel and Shen, 2009). As negative regulator with opposed function to SYD2, the phenotype of rsy-1 LF mutants should be similar to syd-2 GF mutants as discussed by Patel and Shen. The rsy-1(wy94) allele exhibits a point mutation leading to an early stop codon (Q180Stop) and was proposed to be an rsy-1 null (Patel and Shen, 2009). As suggested, rsy-1(wy94) LF mutants showed a tendency toward longer DPs compared to wild type (Fig. IV.31). However, this tendency was not statistically significant. I hypothesized, that a combination of the positive signal of SYD-2 GF with the loss of the negative signal of RSY-1 may lead to strongly elongated DPs. Indeed, analysis of NMJ DP length in cholinergic motor neurons of syd-2(ju487) GF; rsy-1(wy94) LF double mutants revealed very long DPs up to 750 nm (Fig. IV.31), whereas the width remained similar to wild type NMJ DPs (data not shown). At the same time, shorter DPs (50-100 nm) could not be observed in these double mutants. These date indicate that the loss of the negative regulator RSY-1 in a syd-2 GF background enhances the assembly of larger DPs promoted by SYD-2 GF. 


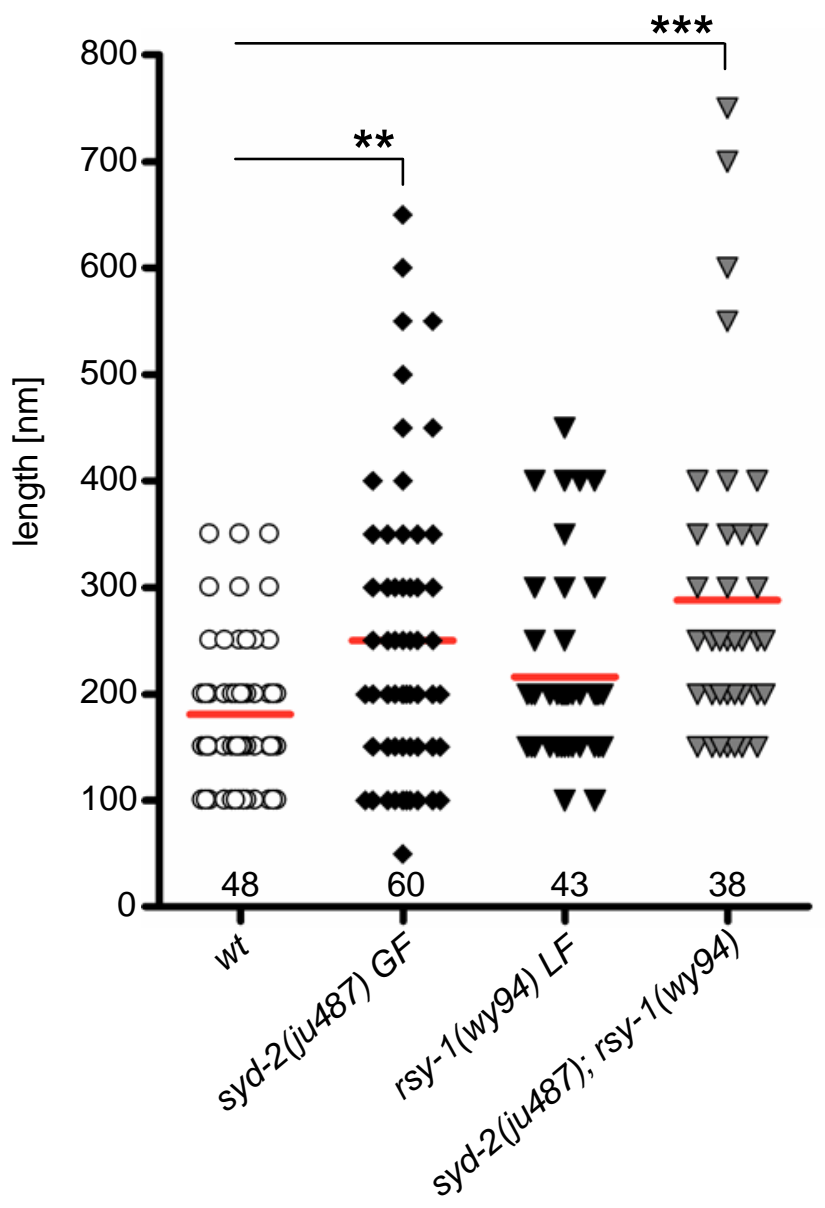

Fig. IV.31 Loss of the negative regulator rsy-1 enhances the formation of elongated NMJ DPs in syd-2 GF mutants. DP length along the axons was analyzed from $50 \mathrm{~nm}$ serial cross sections. Single rsy-1(wy94) LF mutants show a slight tendency for longer DPs compared to wild type. The DP phenotype of syd-2(ju487) GF mutants is enhanced in syd-2(ju487) GF; rsy-1(wy94) LF double mutants, which show DPs up to $750 \mathrm{~nm}$ length. The mean is indicated by the red line. $\mathrm{N}$ is indicated below data points. Statistics: Kruska-Wallis test and Dunn's multiple comparison test. $\left({ }^{*} \leq 0.05\right.$; $\left.* * \leq 0.01 ;{ }^{* * *} \leq 0.001\right)$.

Serial section reconstruction of the ventral nerve cord demonstrates prominent NMJ DPs in syd-2 GF; rsy-1 LF double mutants. The general axon morphology is normal and SVs cluster around the DPs comparable to wild type (Fig. IV.32). 


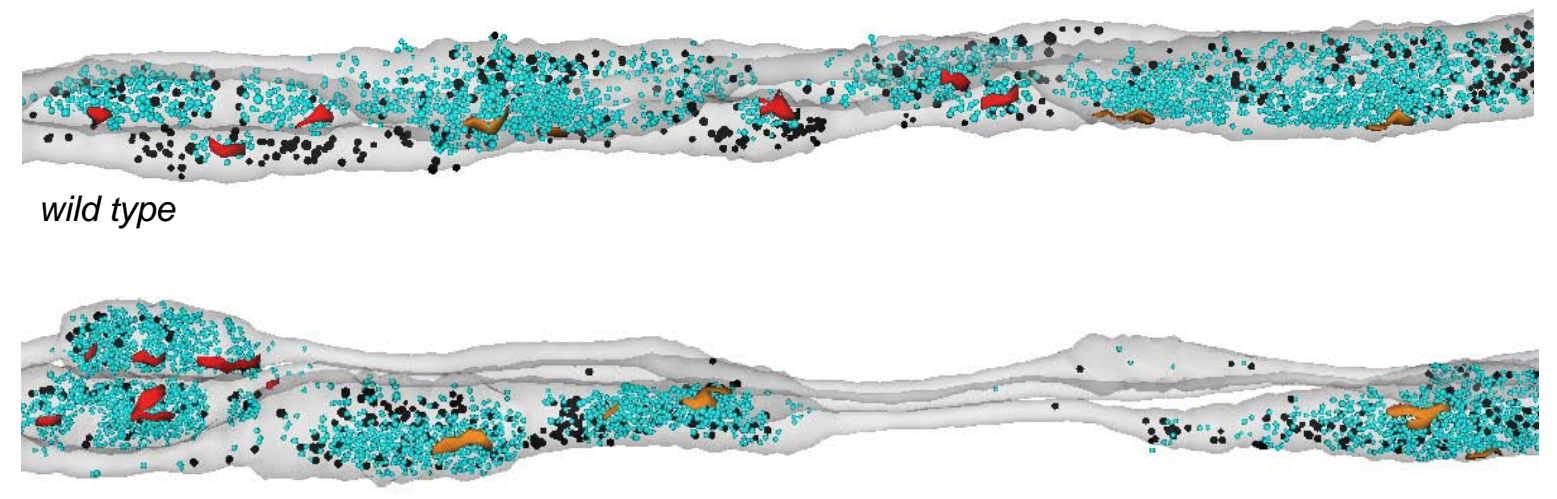

syd-2 (ju487)

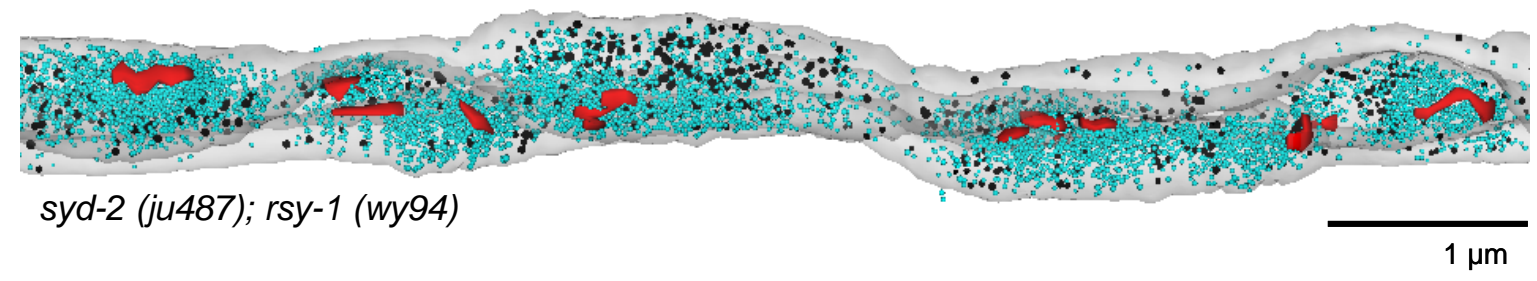

Fig. IV.32 3D Reconstructions of GABAergic and cholinergic motor neurons in the ventral cord of syd-2 GF; rsy-1 LF double mutants. Axon membranes (gray), cholinergic neuron DPs (red), GABAergic neuron DPs (orange), SVs (light blue) and DCVs (black) were reconstructed over $\sim 8 \mu \mathrm{m}$. NMJ DPs are prominent and elongated in syd-2(ju487) GF; rsy-1(wy94) LF double mutants. Vesicles are localized in typical clusters around DPs. Longer DPs seem to recruit more SVs to synaptic terminals.

Quantification of docked and total SV and DCV numbers per terminal and electrophysiological recordings of the single and double mutants have yet to be done to elucidate whether strongly enlarged DPs are able to recruit and dock more vesicles compared to smaller wild type NMJ DPs.

\subsection{The size of NMJ DPs is regulated by counteracting regulators}

Although many $A Z$ proteins have been demonstrated to affect synapse assembly and synaptic transmission, my ultrastructural analysis showed that loss of most $A Z$ proteins can not or only moderately disrupt NMJ DP formation and gross morphology in C. elegans. Except for syd-2 LF mutants, I did not observe a distinct DP phenotype in any of the mutants (Fig. IV.33), indicating that there might be functional redundancy between AZ proteins. RIM-1/UNC-10 was shown to be part of the DP 
(Wang et al., 1997), but it is likely to contribute to the DP fine structure in form of tethers rather than to its size (Stigloher et al., 2011) (Fig. IV.33).

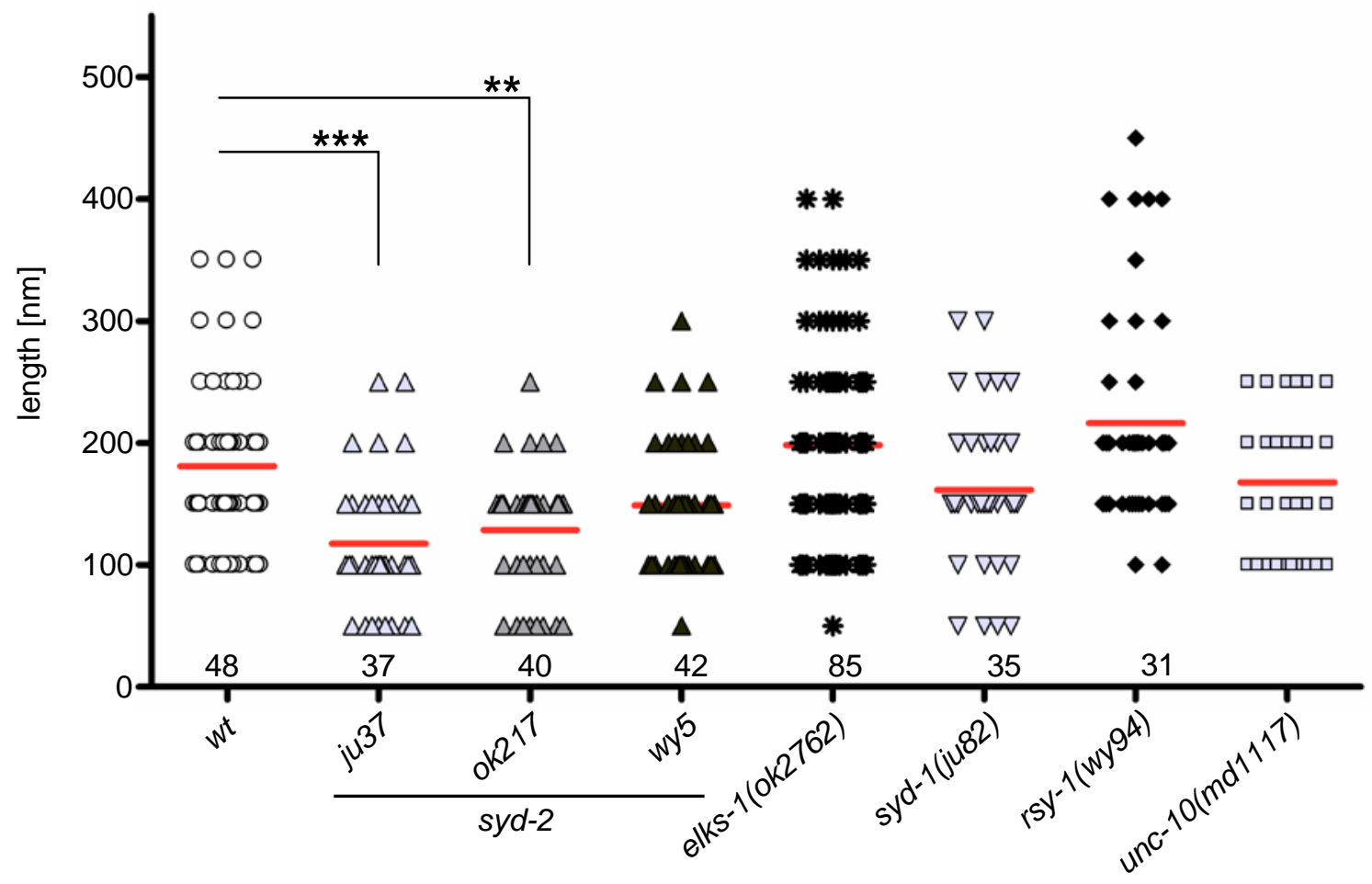

Fig. IV.33 LF mutations in genes known to encode $A Z$ proteins do not affect NMJ DP morphology in $\boldsymbol{C}$. elegans. DP length along the axons was analyzed from $50 \mathrm{~nm}$ serial cross sections. In contrast to syd-2 LF mutants, the LF mutants for the AZ proteins ELKS-1, SYD-1 and UNC-10 as well as the negative regulator RSY-1 showed no significant phenotype in NMJ DP length compared to wild type. The mean is indicated by the red line. $\mathrm{N}$ is indicated below data points. Statistics: Kruska-Wallis and Dunn's posttest with selected pairs $\left({ }^{*} \leq 0.05 ;{ }^{* *} \leq 0.01 ;{ }^{* * *} \leq 0.001\right)$.

In this study, I showed that SYD-2 and RSY-1 are positive and negative regulators of AZ assembly, respectively. They specifically affect DP morphology, most notably DP length. ELKS-1 was confirmed as an additional positive regulator of DP size acting through SYD-2. I propose RSY-1 as a negative regulator, specifically limitating average NMJ DPs size to about 150 to $200 \mathrm{~nm}$. Loss of this limitation signal allows enlargement of the DP structure, especially when combined with enhanced assembly activity of SYD-2 R184C.

I propose that SYD-2 regulates DP architecture and complexity as a positive regulator in concert with ELKS-1 rather than regulating the assembly itself. 


\section{Discussion Chapter 1}

The active zone (AZ) of presynaptic terminals is a complex network of interacting proteins regulating the triggered release of synaptic vesicles (SV). An electron dense projection (DP) can be found in the center of most AZs, yet its exact function, molecular composition and 3D structure remains unclear for most synapse types. The nematode $C$. elegans and its neuromuscular junction (NMJ) have been used as a model system for many studies concerning synaptogenesis and the regulation of synaptic transmission. Yet, investigations of its 3D architecture are limited. In this study, I took advantage of the high-pressure-freezing (HPF) and freeze-substitution (FS) technique that allows ultrastructural preservation close to native state for small samples such as $C$. elegans and combined it with electron microscopy (EM) tomography. I provide a new characterization of $C$. elegans NMJ DPs architecture and shed some light on proteins regulating its size.

\subsection{A branched DP structure can provide a microenvironment for vesicle fusion.}

The description of the $C$. elegans NMJs as rather simple and small plaque-like structure goes back to the serial section reconstructions of the $C$. elegans nervous system by John White (White et al., 1986). Since then, preparation and imaging techniques as HPF and EM tomography have been developed allowing a highresolution analysis of AZ morphology. In this study, I show that AZ DPs in NMJs and also neuron-neuron synapses are not simple, but usually exhibit at least one branch point in the center from which three branches extend rather symmetrically into the $X$ and $Y$ plane (Fig. IV.4). Between these branches, bay-like slots are formed in which SV were frequently found close to or in direct contact with the plasma membrane. I therefore propose that these bays are designated release site where SVs fuse and release their neurotransmitters. C. elegans NMJs are rather small, on average 200 $\mathrm{nm}$ in length and usually have only one or two branch points, which allow the formation of three to four bays (Fig. V.1). 
neuromuscular junction

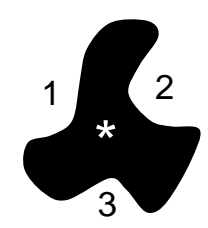

neuron-neuron synapse
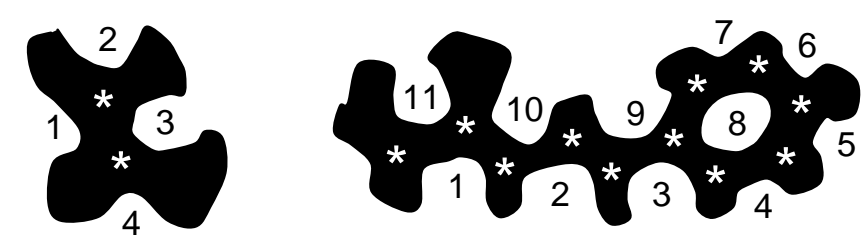

Fig. V.1 Bay structure in C. elegans DPs. Left: NMJ DPs usually exhibit one or two branch points (asterisks) from which small branches symmetrically extend. Three to four bays are formed between these branches and are proposed vesicle fusion sites (numbered). Right: longer neuron-neuron synapses have similar but more branch points that are evenly spaced and accordingly form more bays.

The symmetric character of the DPs becomes even more apparent in longer neuronneuron synapse DPs. Branch points occur periodically within the structure and branches extend symmetrically, forming evenly spaced bays (Fig. V.6). The structural similarity indicates a common assembly principal as well as common structural components between NMJs and neuron-neuron synapses. I hypothesize that the smallest NMJs represents a single DP building unit and that longer DPs are polymers of single units assembled together. This architectural principle is not unique to C. elegans. Tangential electron micrographs of Drosophila T-Bars, similar to the longitudinal sections presented in this work, have revealed an $\mathrm{X}$ or $\mathrm{Y}$ - shaped base, highly similar to the proposed $C$. elegans single building unit (Fig. V.2) (Feeney et al., 1998, Wichmann and Sigrist, 2010).

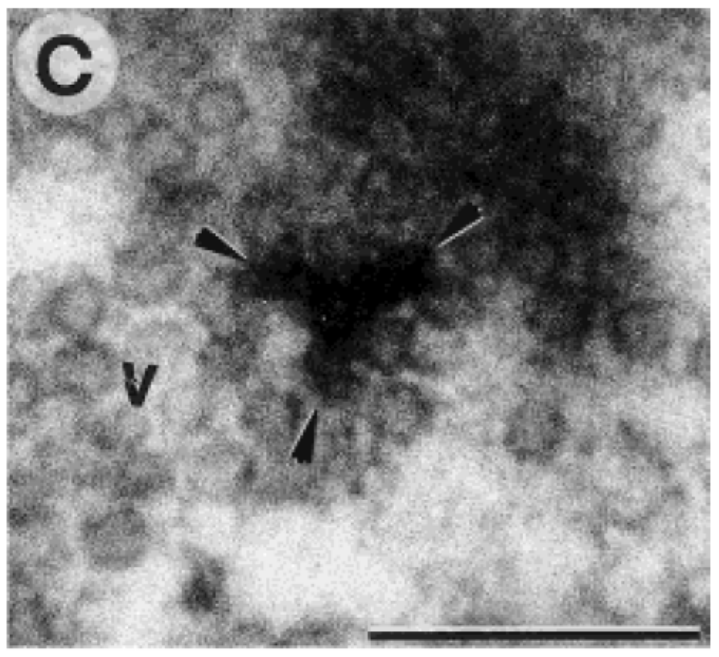

Fig. V.2 Tangential view of the presynaptic NMJ dense T-bar base in the larvae of the flesh flie Sarcophaga bullata. Similar to the NMJ DP structure in C. elegans, the base of the AZ T-bar in motor nerve terminals shows a branched structure (arrowheads) with SVs ( $v$ ) in the bays. Reprinted from (Feeney et al., 1998) with permission. 
The base of the T-bar similarly provides bay-like slots for SV fusion in close proximity to the calcium channels recruited by the T-bar component Bruchpilot. The localization of calcium channels to fusion sites it critical to ensure that, upon stimulation, the calcium influx can reach the primed SVs. The SV-associated calcium sensor synaptotagmin then triggers vesicle fusion with the plasma membrane (FernandezChacon et al., 2001).

Similar to Bruchpilot, other AZ proteins have been shown to contribute to the DP structure or recruit other synaptic proteins. In C. elegans, the Liprin- $\alpha$ homolog SYD2 has been implicated in recruiting many other AZ proteins like ELKS-1 and RIM-1 and SV proteins as RAB-3 and SNB-1 to the AZ. Loss of function studies also suggest that SYD-2 together with RIM-1 contributes to the formation of tethers emanating from the DP (Stigloher et al., 2011). The exact function and structural composition of these tethers is unknown, but it is likely that they contact nearby SVs to recruit them to the fusion sites. Loss of these tethers has been shown to result in reduced evoked response, possibly due to the inefficient recruitment of SVs to the $A Z$ plasma membrane.

According to ultrastructural studies, most $A Z$ components including the fusion machinery are likely to be located within or close to the branches of the DP surrounding the bays (Wang et al., 1997, Tao-Cheng et al., 2000, Yeh et al., 2005, Weimer et al., 2006). Calcium channels are recruited to the DP (Kittel et al., 2006, Gracheva et al., 2008) and tethers can guide nearby SV to the fusion sites. I therefore propose that a microenvironment is created within those bays that can tightly regulate triggered SV fusion. Although NMJ DP architecture varies tremendously between synapse types and organisms, their structure serves the same purpose: local recruitment of the fusion machinery and guidance of SVs to specific fusion sites in the AZ membrane via tethers or, in the case of sensory neurons, via large ribbons or spherical projections. The "particle web" DP in mammalian CNS synapses may have a different geometry, but similarly provides designated fusion slots for single SVs surrounded by an AZ protein scaffold (Phillips et al., 2001). Size and complexity are likely to resemble adjustments to the type of release and size of the readily releasable pool (RRP).

The RRP, that is the pool of vesicles rapidly fusing upon onset of high frequency or hyperosmotic stimulation, has been shown to constitute a surprisingly small percentage of the total SV pool in many synapses (reviewed in Rizzoli and Betz, 
2005). One electrophysiological study in C. elegans estimated the RRP in wild type C. elegans to contain around 18 vesicles (Gracheva et al., 2006). It has to be noted that, due to the small size of $C$. elegans neurons, RRP measurements are whole cell recordings collected from muscle cells. The RRP therefore does not resemble the RRP of one but several cholinergic synapses (personal communication Erik Jorgensen). It can be assumed that the structure of the C. elegans DP is directly correlated to its function. Therefore I propose that only 3 to 4 vesicles may be readily releasable per cholinergic terminal and that these vesicles are located in the bays of the DP.

The proposal of a single DP building unit raises the question how specific entities of $A Z$ proteins are assembled. Studies in hippocampal neurons have identified specific dense core vesicles (DCVs) associated with AZ proteins Piccolo and Bassoon, socalled Piccolo-Bassoon transport vesicles (PTVs). Quantitative immuno-fluorescence analysis revealed that each vesicle contained about half of the amount of Bassoon, Piccolo and RIM protein that is found in individual presynaptic terminal (Shapira et al., 2003). It was proposed that AZs are generated from two to three of these PTVs (Shapira et al., 2003). A similar proposal has been made for photoreceptor ribbon synapses in the mouse retina. Electron dense precursor spheres were shown to be associated with the ribbon DP proteins Piccolo, Bassoon, RIBEYE and RIM1. These precursors were transported to nascent release sites during synaptogenesis to form synaptic ribbons (Regus-Leidig et al., 2009). It is conceivable that AZ transport organelles in C. elegans - although not yet identified - contain specific AZ protein entities and DP single units are assembled from a defined number of such organelles or vesicles.

\subsection{SYD-2 and RSY-1 are counteracting proteins that define NMJ DP size}

\subsubsection{SYD-2 determines the length of NMJ DPs}

To build such a highly structured NMJ, organized assembly of AZ proteins has to occur. Many genetic and biochemical interactions have been identified between known AZ components. Yet, their precise function remains largely unclear. A hierarchical assembly has been proposed for hermaphrodite specific neurons (HSN) 
in C. elegans. Upstream regulators recruit downstream effectors to nascent synapses. SYD-2 /Liprin- $\alpha$ has been identified as one of the major assembly molecules. Loss-of-function (LF) mutants of SYD-2 have been shown to exhibit longer NMJ DPs compared to wild type (Zhen and Jin, 1999). I performed a similar ultrastructural EM analysis of $C$. elegans syd-2 LF mutants, including the syd-2(ju37) allele analyzed by Zhen and Jin. However, the severe truncation of SYD-2 in allele ju37 and presumed loss of SYD-2 in allele ok217 lead to significantly shorter DPs in our preparations. I explain this contradicting data with the different sample preparation methods used for EM. Zhen and Jin prepared the animals via classical chemical fixation, which possibly allowed DP material and other components to move and diffuse during the rather long fixation and dehydration procedure (Zhen and Jin, 1999). In this study, I was able to preserve the ultrastructure in an almost native state by applying HPF and FS, which allows evaluation of DP size in a more reliable and reproducible way. Both syd-2 LF alleles analyzed showed similarly reduced DP size and reduced number of overall SVs and DCVs, suggesting that severe truncation or complete loss of SYD-2 leads to inefficient recruitment of AZ proteins (Fig. IV.9 and IV.12).

In contrast, a syd-2 gain-of-function (GF) mutation leads to the formation of elongated DPs. This GF mutation has been proposed to be more active and thus able to bypass the requirement of upstream regulator SYD-1 (Dai et al., 2006). Overexpression of wild type SYD-2 has been reported to mimic the SYD-2 GF phenotype (Dai et al., 2006, Patel et al., 2006). It has yet to be determined whether SYD-2 overexpression also leads to the formation of elongated DPs in C. elegans NMJs. This would indicate that the amount of SYD-2 directly determines the length of the DPs. It is interesting to note that elongated DPs at NMJs in SYD-2 GF mutants seem to be similar polymers of DP single building units as observed in wild type neuron-neuron synapses, indicating a similar assembly principle (Fig. IV.10).

It is not yet clear how the exchange of an arginine with a cysteine in the LH1 domain results in a gain of function, but it is possible that a cystein at this position forms a disulfide bond with a second SYD-2 GF molecule, leading to a stable SYD-2 GF dimer. The formation of dimers and higher oligomers through the SYD-2 LH1 domain has just recently been proposed to enhance SYD-2 activity in promoting presynaptic assembly (Taru and Jin, 2011). It is therefore conceivable that more stable SYD-2 dimers may recruit more AZ proteins to form longer DPs. These interactions may be 
direct or indirect through binding of UNC-104. Together with the data presented in this work, I suggest that SYD-2 is involved in regulating the size of DPs rather than their assembly per se.

\subsubsection{ELKS-1 enhances SYD-2 function}

Evidence for stronger interaction of the SYD-2 GF N-terminus with a second SYD-2 protein or the AZ protein, ELKS-1, was provided in this work. It had previously been shown that ELKS-1 needs to be present for SYD-2 GF to bypass the loss of upstream regulator SYD-1. We could narrow down the interaction site to the LH1 domain of SYD-2 by a yeast-two-hybrid screen and in vitro pull-down assays (Fig. IV.25 and IV.26). By analyzing AZ morphology of syd-2 GF; elks-1 LF double mutants we could also show that ELKS-1 is needed in vivo for the formation of elongated DPS in the syd-2 GF background (Fig. IV.29). As ELKS-1 single mutants do not show a clear behavioral or morphological phenotype, the question remains why ELKS-1 interaction with SYD-2 GF is needed for SYD-2 GF functionality.

SYD-2 activity has been shown to depend on its upstream regulator SYD-1, which in turn has been shown to facilitate interaction of ELKS and SYD-2 (Patel and Shen, 2009). It is conceivable that both proteins can activate SYD-2 function, possibly by promoting dimer formation or a conformational change. When only ELKS-1 is lost, SYD-1 may compensate the loss of ELKS-1 function and therefore no phenotype is observed in elks-1 LF mutants. However, in the syd-2 GF background, SYD-1 alone is not sufficient to promote SYD-2 activity leading to increased recruitment of $A Z$ material. Therefore, the strongly elongated DPs are lost, but wild type size DP formation still occurs. If both positive regulators are lost, SYD-2 and SYD-2 GF functionality is strongly compromised as shown by impaired HSN synapse assembly (Dai et al., 2006). It has yet to be determined whether loss of SYD-1 and ELKS-1 together has a similarly strong phenotype in C. elegans motor neurons. It is conceivable that SYD-1 enhances SYD-2 activity directly and through facilitating ELKS-1 interaction with SYD-2. This interaction possibly makes SYD-2 more accessible for dimerization and interactions with components involved in synapse assembly. 


\subsubsection{RSY-1 counteracts SYD-2 function in defining DP size}

If positive regulators like SYD-2 and ELKS-1 enhance AZ formation, there must be counteracting negative regulators as well. Not just DP size has to be limited, but also the formation of ectopic synapse formation in dendrites, axon, commissures or the cell body. Only few of these negative regulators have been identified. In C. elegans, ectopic formation of synapses in dendrites is prevented by axon guidance cues like UNC-6/netrin and its receptor UNC-5 (Poon et al., 2008). Wnt signaling pathways have been shown to spatially restrict synapse formation to a specific segment of motor neuron axons (Klassen and Shen, 2007). Besides the correct localization of synaptic sites, their size and number have to be defined. The regulator of presynaptic morphology (RPM-1) is homologous to Drosophila Highwire and mammalian Pam and has been shown to restrict the size of presynaptic terminals during the development of GABAergic motor neurons in C. elegans (Zhen et al., 2000).

In Drosophila, it was proposed that the anaphase-promoting complex/cyclosome $(\mathrm{APC} / \mathrm{C})$ regulates synapse size and number by modulating levels of its downstream effector Liprin- $\alpha$ by local ubiquitin-mediated degradation (van Roessel et al., 2004).

A visual genetic screen in $C$. elegans recently identified a new negative regulator of synaptogenesis (RSY-1) (Patel and Shen, 2009), which was shown to directly interact with SYD-1 and SYD-2 in vitro and in vivo. Protein interaction assays in HEK cells provided evidence that RSY-1 negatively affects SYD-2 functionality. This negative regulation was proposed to be direct as well as indirect by impairing SYD-1 binding to ELKS-1, possibly affecting the ability of SYD-1 to enhance SYD-2 function. The connection between RSY-1, ELKS-1 and the key assembly molecules SYD-1 and SYD-2 indicates that they could cooperate as components of a local machinery regulating DP formation and size. Indeed, I was able to show that the absence of RSY-1 allows formation of slightly elongated DPS (Fig. IV.31). Combining the enhanced positive signal of SYD-2 GF and the lack of negative signal of RSY-1 in a syd-2 GF; rsy-1 LF double mutant allowed the formation of greatly enlarged DPs (Fig. IV.31). Further experiments will have to be performed to shed light on the functional implications of these findings. Electrophysiological recordings are needed to investigate if synaptic transmission is enhanced when larger DPs are present. In single syd-2 GF mutants we were not able to detect a difference compared to wild type, but the effect could be too small to be detected. Additional EM analysis has to be conducted to demonstrate whether the number of docked SVs increases with 
higher numbers of bays in strongly elongated DPs, which would be in line with enhanced synaptic transmission. Serial EM tomography needs to be conducted to determine if the 3D structure of the enlarged DPs is indeed similar to the complex neuron-neuron synapses.

RSY-1 was shown to interact with the first two SAM domains of the SYD-2 Cterminus, but the binding sites of RSY-1 are unknown and have yet to be determined. The mechanism how RSY-1 impairs SYD-1 and SYD-2 function is not clear. A competitive binding with ELKS-1 to SYD-2 is unlikely as RSY-1 was suggested to interact with the SAM domains (Patel and Shen, 2009). Mammalian ERC/ELKS, however, was shown to bind to the N-terminal coiled coil domains of Liprin- $\alpha$ /SYD-2 (Ko et al., 2003b). If SYD-2 exists in an active and inactive state, it is conceivable that RSY-1 might stabilize the inactive state, counteracting the function of SYD-1 and ELKS-1. If this was true, analysis of DP size in syd-1; elks-1 double mutants should show a similar reduction of DP size as syd-2 LF mutants. Investigations of hermaphrodite specific neurons (HSN) in the same mutants have been performed in C. elegans, but cannot easily be consulted to clarify the precise regulatory mechanism, as single mutant phenotypes are already stronger than in NMJ synapses (Dai et al., 2006). Synapse assembly regulation in HSN and NMJs may be adjusted differently and one has to be cautious to draw conclusions about NMJ DP assembly from these findings and vice versa.

\subsubsection{Interaction of SYD-2 with kinesin UNC-104 as mechanism to alter DP size}

Other interactions with SYD-2 that may be involved in DP assembly include binding to synaptic proteins like RIM-1, GIT, LAR and also to kinesin-3 UNC-104 (SerraPages et al., 1998, Schoch et al., 2002, Ko et al., 2003a). Interestingly, SYD-2 was shown to enforce anterograde movement of UNC-104 in C. elegans (Wagner et al., 2009). Direct interaction of $\mathrm{N}$-terminal coiled coil domains of the mammalian SYD-2 homolog Liprin- $\alpha$ with the Liprin-binding domain in the UNC-104/KIF1A stalk region had already been demonstrated in vitro (Shin et al., 2003). Wagner and colleagues could additionally show strong interaction of the SYD-2 C-terminus, including the SAM domains with the UNC-104 stalk domain. Two possibilities arise from these findings: (1) SYD-2 could promote UNC-104 dimerization and thus turn UNC-104 into 
a more processive motor in anterograde direction (Tomishige et al., 2002). (2) Binding of SYD-2 to UNC-104 could affect recruitment of synaptic proteins from the cell body to nascent synapses at two check points during the transport: in the cell body where SYD-2 could act as an adaptor protein between synaptic protein transport vesicles and UNC-104; or at synaptic sites where binding to UNC-104 could trigger cargo unloading. While the precise role of this interaction has yet to be determined, SYD-2 binding to UNC-104 is clearly important for efficient synapse assembly (Miller et al., 2005). An unc-104 mutant with a defective Liprin-binding domain would be a great tool to study the role of SYD-2 interaction with UNC-104. So far, no such mutant has been identified in C. elegans. Possibly, because the phenotype is too weak to be select in a mutagenesis screen or too strong so mutants are not viable. Generation of an UNC-104 construct with a mutated Liprin-binding domain by PCR mutagenesis is a possibility to address the question. However, we would be unable to provide a clean background in which this construct could be expressed, because unc-104 null mutants are not viable. Therefore, in vivo studies in C. elegans with this construct are not feasible. Nevertheless, in vitro analysis of synaptic protein localization and protein transport in neuron cell culture expressing this construct could provide valuable insight into the function of SYD-2 interaction with UNC-104 kinesin during synapse and DP assembly.

\subsubsection{Synaptic plasticity via regulation of DP size}

The presence of counteracting proteins that can dynamically regulate DP size must have a functional implication. The fact that the nervous system of $C$. elegans is highly stereotypic also means that neuronal plasticity is probably not mediated by addition or elimination of synapses or neurons, but rather dynamic alterations of synapse strength. This synaptic plasticity can either occur during development or postdevelopmentally in adult animals as a means of network homeostasis and scaling. I propose that SYD-2, ELKS-1 and RSY-1 play a major role in regulating the NMJ DP size to adjust synapse strength by providing more or less vesicle fusion sites (Fig. V.3). 


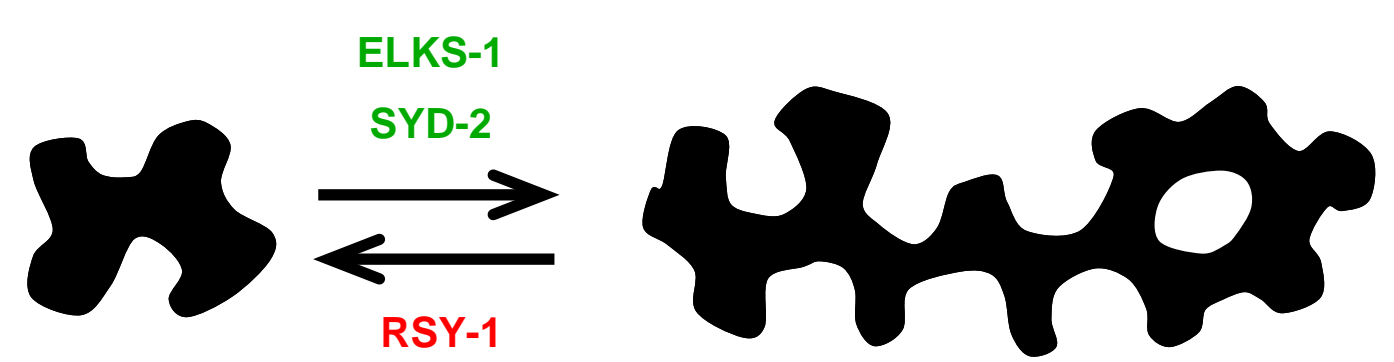

Fig. V.3 Positive and negative regulators control DP size. Counteracting AZ proteins ELKS, SYD-2 and RSY-1 are thought to cooperate to define DP length at NMJ in C. elegans. ELKS-1 and SYD-2 promote the elongation of DP size, whereas RSY-1 limits DP size by negatively affecting SYD-2 functionality.

Under normal conditions, cholinergic NMJ DP size is limited to an average of 150$200 \mathrm{~nm}$ (Fig. IV.9). If needed, activity of negative or positive regulators can be increased or decreased. This may be accomplished by regulating their expression levels, via degradation (Hoogenraad et al., 2007), ubiquitination (van Roessel et al., 2004) and many other molecular modifications. As discussed above, Drosophila Liprin-a/SYD-2 levels were shown to be regulated by the anaphase-promoting complex/cyclosome (APC/C) (van Roessel et al., 2004). In the C. elegans genome, $15 \mathrm{APC} / \mathrm{C}$ orthologs have been identified so far (Shakes et al., 2011). It is therefore imaginable that this mechanism is conserved and might be utilized to adjust DP size. Reasons for alteration of synaptic strength could be environmental stress such as continuous stimuli by chemical or mechanical cues. C. elegans has also been shown to be able to learn and associate their cultivation temperature with food (Hedgecock and Russell, 1975). This process may be mediated by adjustment of synaptic transmission in thermosensory neurons, possibly via regulation of DP size.

The $A Z$ of every synapse is a highly complex regulatory network that organizes the delicate processes of triggered SV fusion to transmit a very specific signal to a postsynaptic target cell. An important element of this network is the DP that brings all components of the fusion machinery into close proximity and thus ensures efficient neurotransmitter release. In this study I shed light onto the mechanisms involved in the formation and regulation of the characteristic AZ DP by the counteracting proteins SYD-2, ELKS-1 and RSY-1 in vitro and in vivo in the nematode C. elegans. 


\section{Introduction Chapter 2}

Neurons are highly polarized cells with an axon responsible for signal transmission and one or more dendrites for signal reception and integration. Accordingly, different organelles and proteins have to be transported from the cell body to the respective compartment. For instance, neurotransmitters and active zone (AZ) proteins for the release machinery have to be delivered to the axons while receptors have to be transported to the dendrites. In general, this transport is believed to occur in vesicular transport carriers that are transported along microtubule tracks, probably via the action of ATP-dependent motor proteins (Fig. VI.1). In vertebrate axons, microtubules are oriented with their plus-ends towards the synapse, and with their minus-ends towards the cell body. Kinesins are believed to generally mediate anterograde axonal transport from the cell body to synapse while dyneins and minusend directed kinesins generate retrograde transport from synapse to cell body (reviewed in Goldstein and Yang, 2000).

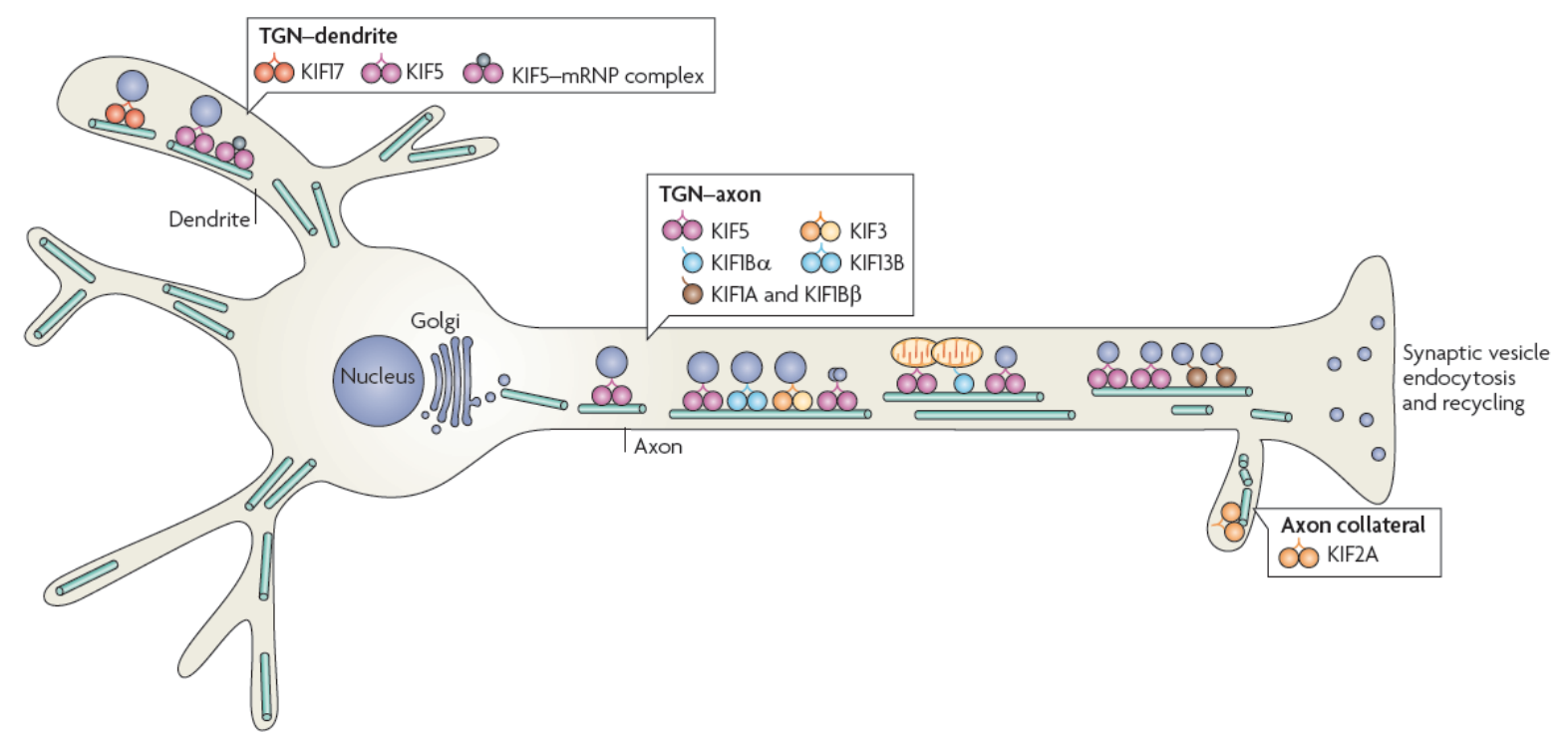

Fig. VI.1 Intracellular transport by different kinesin motors in neurons. In neuronal axons microtubule plus ends always point distally. Plus end-directed motors therefore transport their cargos to the cell periphery. Different kinesin families are indicated. They are responsible for axonal transport to the synapse and into the dendrite, respectively. Reprinted with permission from Macmillan Publishers Ltd: Nature Reviews Molecular Cell Biology, (Hirokawa et al., 2009), copyright (2009). 


\subsection{The kinesin family of motor proteins}

Kinesins were first identified in the giant axons of squids as proteins that induce microtubule-based movements, yet with different enzymatic behavior and molecular weight than myosin and dynein. Vale and colleagues therefore introduced the name kinesin (Vale et al., 1985). They can be divided into subfamilies according to sequence similarity, domain organization and motility properties. To date, 18 different kinesins were found to be encoded in the Caenorhabditis elegans genome. (http://www.cellbio.duke.edu/kinesin/OrgTables/caeel.html hosted by the Department of Cell Biology at Duke University). UNC-104/KIF1A is a neuron-specific kinesin that has been proposed as key anterograde motor for dense core vesicle (DCV) and synaptic vesicle (SV) transport (Hall and Hedgecock, 1991). It is a member of the kinesin-3 subfamily and was originally identified in C. elegans (Ohtsuka et al., 2002). Homologs are present in Drosophila (Imac; Pack-Chung et al., 2007) and mouse (KIF1A; Okada et al., 1995)

UNC-104 features an N-terminal motor domain with an ATP binding pocket, facilitating ATP-dependent movement along the microtubules (Fig. VI.2) (reviewed in Vale and Milligan, 2000). The C-terminus of UNC-104 has been shown to recruit cargo vesicles via its pleckstrin homology $(\mathrm{PH})$ domain, which binds to the membrane lipid phosphatidylinositol-4,5-bisphosphate $\left(\mathrm{PI}(4,5) \mathrm{P}_{2}\right)$ (Fig. VI.2) (Klopfenstein et al., 2002, Klopfenstein and Vale, 2004). Coiled coil regions connect the motor and PH domain and are thought to promote dimerization resulting in a more cooperative and faster unidirectional instead of slow a bidirectional movement (Tomishige et al., 2002, Shimizu et al., 2005). 


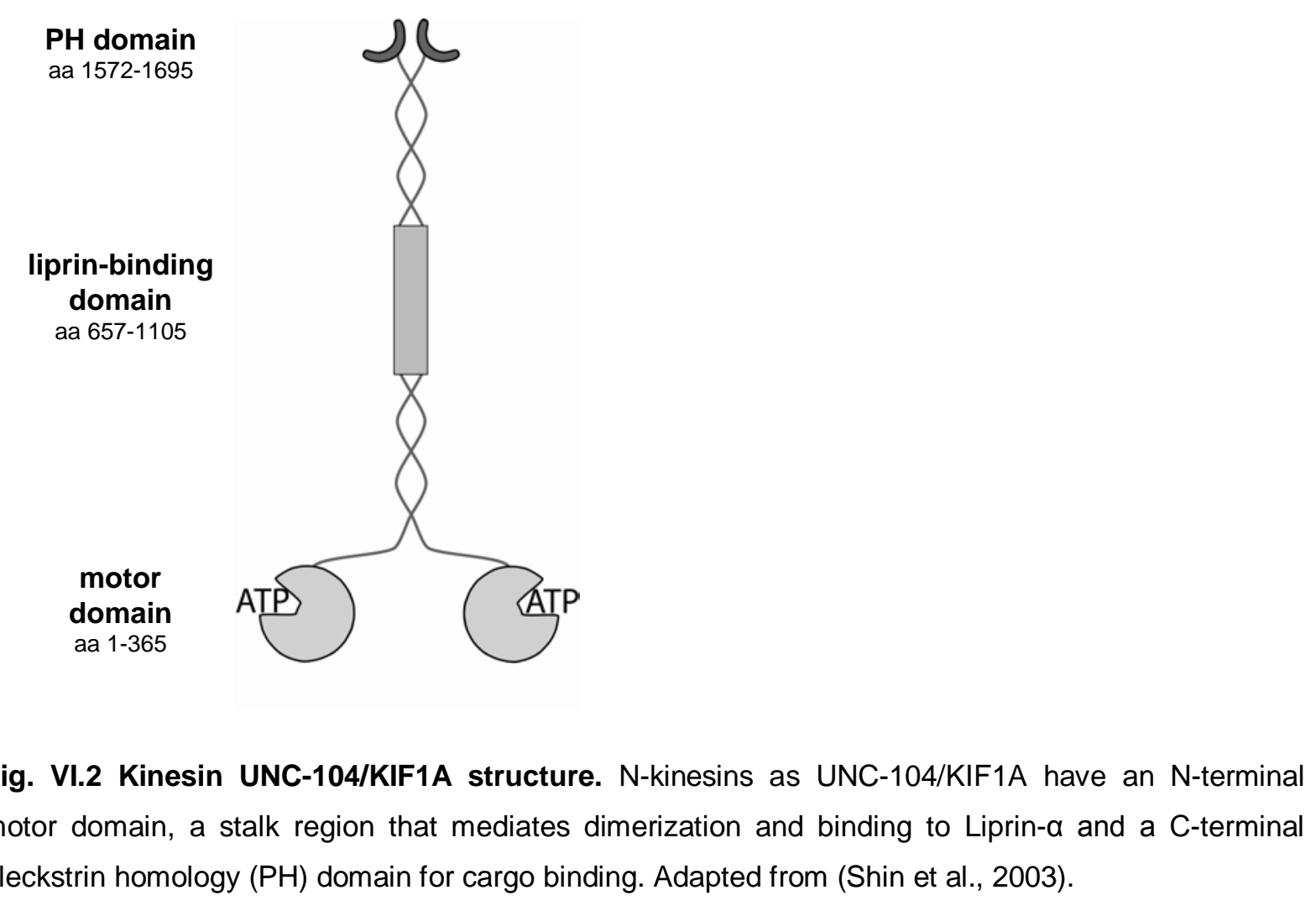

\subsection{Kinesin cargo recognition and unloading}

The precise mechanism how kinesins identify their specific cargo is not fully understood. They can either directly bind to the cargo membrane or via adaptor/receptor proteins that mediate specific binding. Several adaptor proteins have been identified in vertebrates (reviewed in Hirokawa et al., 2009), in Drosophila (Toda et al., 2008) and C. elegans (Byrd et al., 2001, Sakamoto et al., 2005, Chua et al., 2012). An equally unresolved question is how kinesins recognize the correct target site for unloading their cargo. There is some evidence that contact to postsynaptic targets may trigger the formation of exocytosis sites, possibly through transmembrane signaling of adhesion molecules (Hazuka et al., 1999, Bruses, 2000). Subsequent changes in the cytoplasm may be recognized by kinesins, inducing them to stop and release their cargo (Roos and Kelly, 2000). Phosphorylation has been demonstrated for different kinesins as possible regulatory mechanism for loading and uncloading cargo (Guillaud et al., 2008, Chua et al., 2012). 


\subsection{UNC-104 and its function in synaptogenesis}

Loss of UNC-104 in C. elegans impairs axonal localization of fluorescently-tagged proteins associated with SVs and DCVs. (Hall and Hedgecock, 1991, Okada et al., 1995, Zahn et al., 2004). Instead, the cargo accumulates in the cell bodies (Hall and Hedgecock, 1991; Patel et al., 2006). Similar phenotypes were observed in Drosophila kinesin-3 imac mutants (Pack-Chung et al., 2007) and mouse KIF1A mutants (Yonekawa et al., 1998). On the contrary, dense projection (DP) and $A Z$ proteins are usually not or only moderately affected (Hall and Hedgecock, 1991, Zhen and Jin, 1999, Koushika et al., 2001, Sieburth et al., 2005, Patel et al., 2006). This suggests that different proteins are sorted onto different types of transport organelles, which are then recognized by their specific motor protein (Fig. VI.1).

A recent characterization of unc-104(e1265) mutants in C. elegans demonstrated defective binding of $\mathrm{PI}(4,5) \mathrm{P}_{2}$ due to a point mutation in the $\mathrm{PH}$ domain. Reduced cargo-binding ability in turn results in lower levels of UNC-104, suggesting degradation of motors without cargo. In line with this observation, retrograde movement of UNC-104 is limited, indicating that motors are not transported back to the cell body, but degraded upon cargo unloading (Kumar et al., 2010).

UNC-104/KIF1A mutants resemble not just a great tool to investigate intracellular transport per se, but also its role in synaptogenesis. With increasing knowledge about cargo-kinesin specificity, we can use these motor proteins as genetic tool to selectively eliminate transport of distinct synaptic cargo to the axon. Subsequent analysis of behavioral phenotypes and synapse morphology will provide further insight into the mechanisms of synapse assembly and the individual functions of specific synaptic proteins.

How and where SVs are generated has been a controversial topic. It is assumed that they reach the synaptic terminal as precursors and are then locally transformed into mature SVs. However, the nature of these precursor vesicles and the process of their maturation are under debate. In unc-104 mutants these SV precursors are retained in the cell body and accessible for analysis. Previous descriptions of these vesicles are contradicting. Either clear core or dense core vesicles have been observed (Hall and Hedgecock, 1991, Jorgensen et al., 1995). So far, unc-104 mutants have been analyzed mainly via fluorescence microscopy and biochemistry, largely neglecting the ultrastructural aspect of the remaining AZs and the vesicle-filled cell bodies. 
In this study we took advantage of the improved ultrastructural preservation of tissue through high-pressure-freezing and freeze substitution to characterize the synaptic ultrastructure of C. elegans unc-104 mutants. By combining fluorescence analysis and electron microscopy, we analyzed the vesicles retained in unc-104 mutant cell bodies and the morphology of the remaining synaptic terminals. 


\section{Results Chapter 2}

Anterograde transport of synaptic vesicles (SVs) has been shown to be mediated by the neuron-specific kinesin-3 UNC-104/KIF1 (Hall and Hedgecock, 1991). Despite intense investigations in the kinesin field, ultrastructural information about unc-104 mutants are limited. To characterize neuron morphology in C. elegans, I analyzed three unc-104 LF alleles with different mutation sites by combining localization studies of fluorescently-tagged proteins with electron microscopy (EM). The amino acid exchanges G96E and G314E in unc-104(rh43) mutants affect the ATP binding pocket and reduced the functionality of the motor domain (Fig. VII.1) (Kumar et al., 2010).

In unc-104(e1265) mutants, the D1497N amino acid exchange within the pleckstrin homology $(\mathrm{PH})$ domain leads to strongly reduced $\mathrm{Pl}(4,5) \mathrm{P}_{2}$ binding (Kumar et al., 2010), whereas a Q1418Stop mutation in unc-104(ce515) mutants terminates the UNC-104 protein just prior to the $\mathrm{PH}$ domain (personal communication Ken Miller; Fig. VII.1).

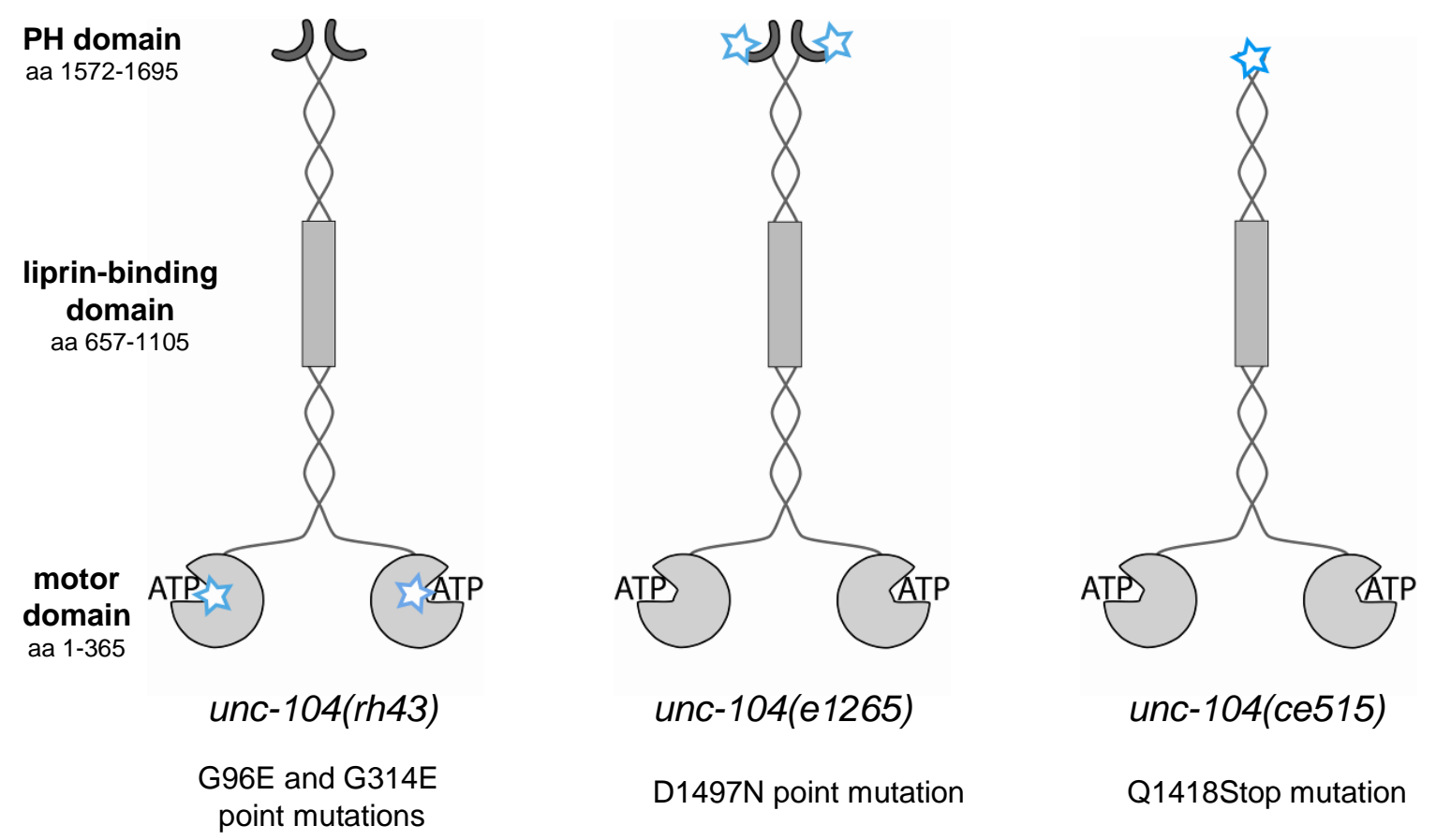

Fig. VII.1 UNC-104 protein domains and mutation sites. Motor domain, Liprin-binding domain and $\mathrm{PH}$ domain are indicated. Mutation sites are indicated within the protein model (star) and described underneath. The mutation in allele rh43 disrupts the ATP binding pocket and therefore affects the 
motor domain of the kinesin. Allele e1265 and ce515 have an affected or missing PH domain and therefore defective cargo binding. Adapted from (Shin et al., 2003).

\subsection{Synaptic vesicles mislocalize to cell bodies and dendrites in unc-104 mutants}

For an initial characterization of the transport defects in all three unc-104 mutants, I expressed fluorescently-tagged SV and DCV marker proteins specifically in cholinergic DA and DB motor neuron under the control of the unc-129 promoter (Sieburth et al., 2005). Dorsal nerve cord and cell bodies were imaged to check for altered SV and DCV localization.

The SV proteins RAB-3 and synaptobrevin (SNB)-1 as well as neuropeptides NLP-21 (Neuropeptide-Like $\underline{\text { Protein) }}$ and INS-22 (type-alpha INSulin-like molecule) are expressed in a clear puncta pattern along the axons in the dorsal cord. Each punctum represents a cloud of SVs or DCVs, respectively. In all unc-104 mutants no fluorescence was detectable in the dorsal cord, suggesting the absence or strong reduction of vesicles at synaptic sites (Fig. VII.2 and Fig. VII.3). 


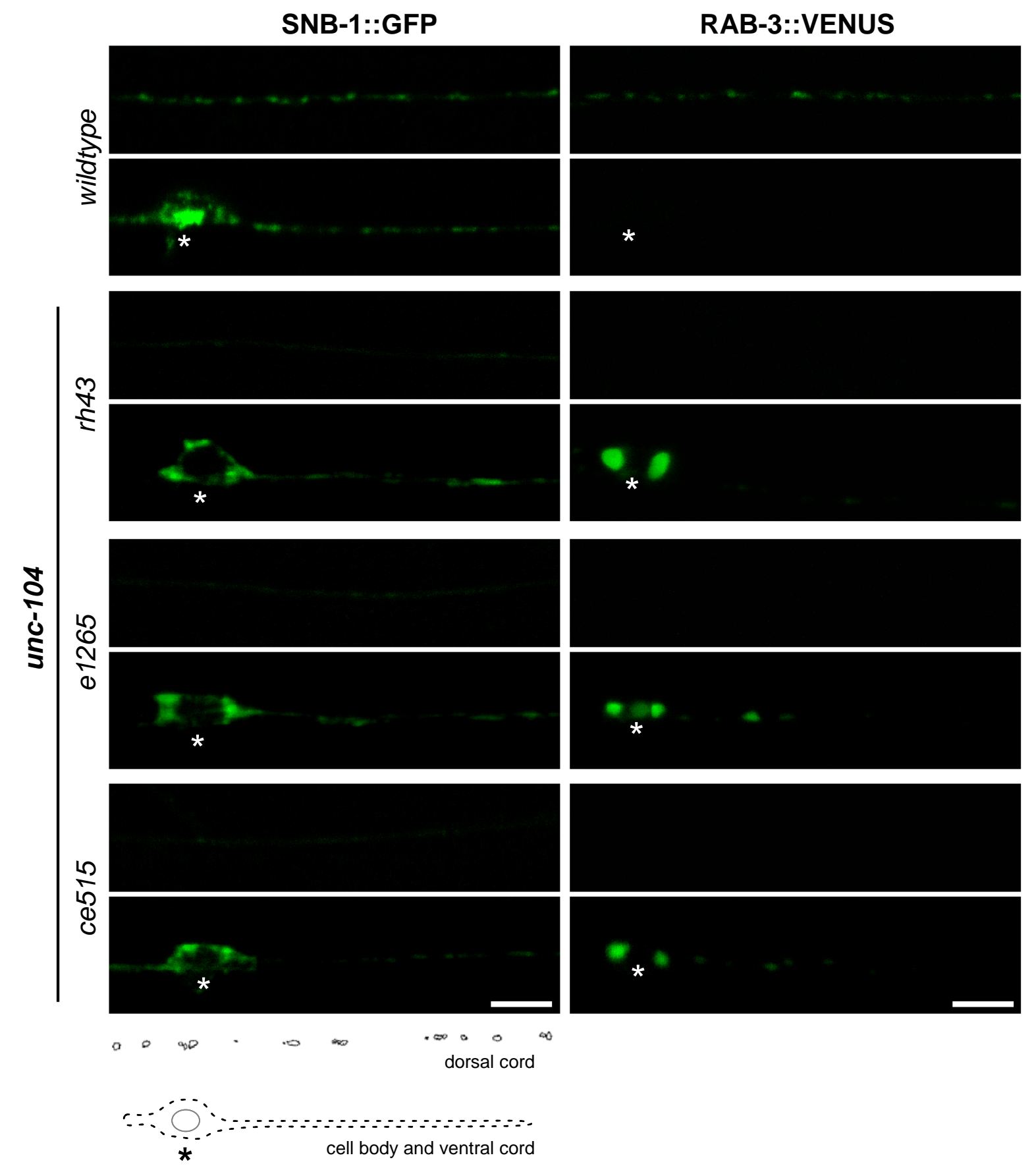

Fig. VII.2 SV marker expression in wild type and unc-104 mutants. SNB-1::GFP and RAB$1::$ VENUS were expressed under the unc-129 promoter. Dorsal cord axons and ventral cord cell body with dendrite are shown as indicated in scheme below. Asterisks mark position of cell body. Fluorescence is lost in axons and accumulates in cell bodies and proximal dendrite in unc-104 mutants. Scale bar is $5 \mu \mathrm{m}$.Crossing of the marker strains into unc-104 mutant background was done by the author and Dr. Christoph Wrede. Imaging was done by the author. 
Instead, fluorescence markers accumulate in unc-104 mutant cell bodies and proximal regions of the dendrites. This phenotype is most apparent for the RAB3::VENUS construct, where fluorescence is absent in the cell bodies of wild type animals. All other markers exhibited fluorescence in wild type cell bodies due to overexpression of the construct. Additionally, some SV and DCV puncta emerge in motor neuron dendrites (ventral nerve cord) in unc-104 mutants (Fig. VII.2 and Fig. VII.3), possibly due to retrograde transport via dynein (Koushika et al., 2004).

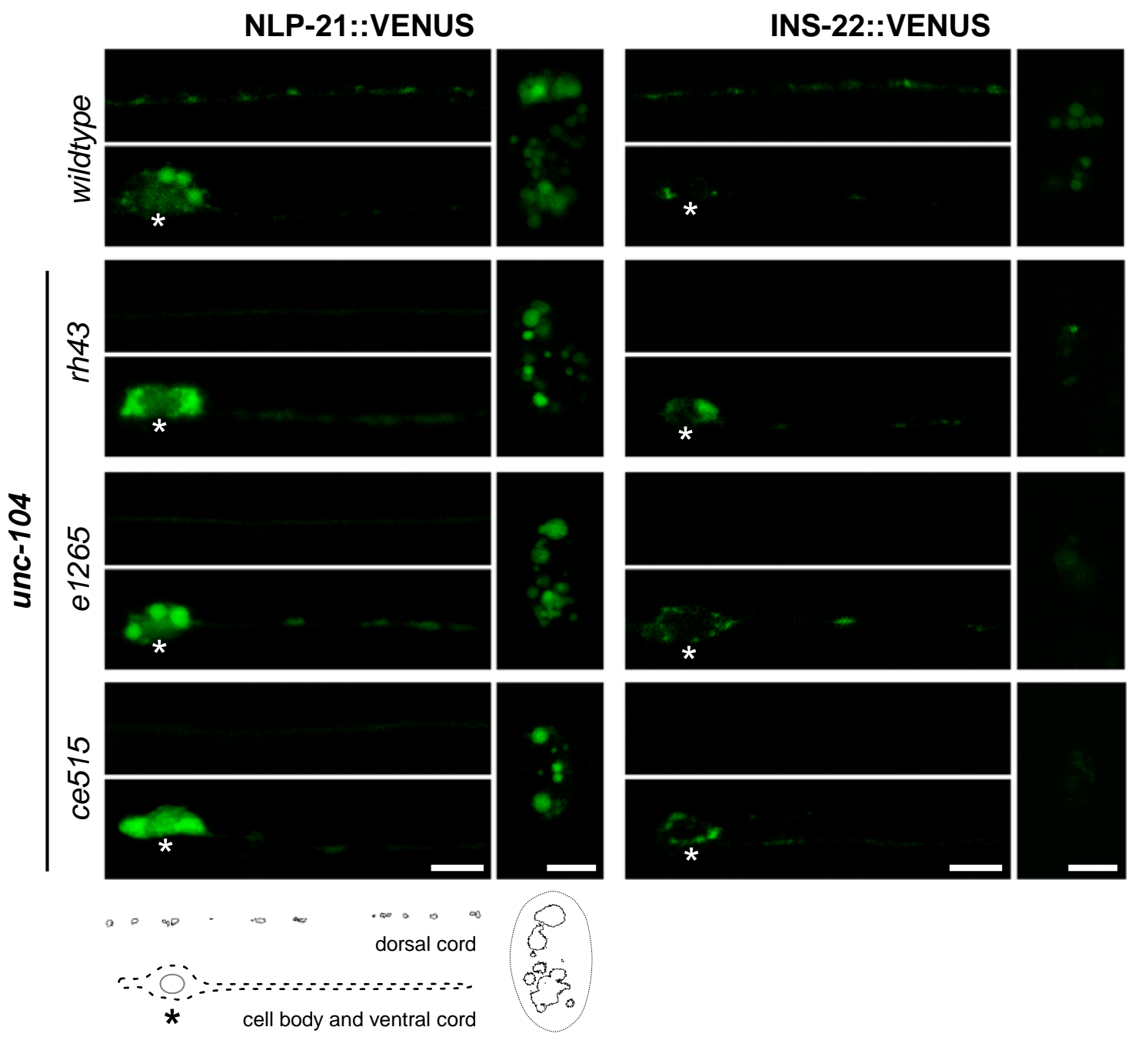

Fig. VII.3 DCV marker expression in wild type and unc-104 mutants. NLP-21::VENUS and INS22::VENUS were expressed under the unc-129 promoter. Dorsal cord (axons), cell body with adjacent ventral cord (dendrites) and coelomocytes are shown as indicated by scheme below. Asterisks mark position of cell body. Fluorescence is lost in axons and accumulates in cell bodies and parts of the dendrites in unc-104 mutants. Secretion of fluorescently-tagged neuropeptides is indicated by fluorescence uptake by coelomocytes. Scale bar is $5 \mu \mathrm{m}$. Crossing of the marker strains into unc-104 
mutant background was done by the author and Dr. Christoph Wrede. Imaging was done by the author.

Fluorescently-tagged neuropeptides are secreted from DCVs into the synaptic cleft and diffuse into the pseudocoelom of $C$. elegans. Eventually they reach the coelomocytes, macrophage-like cells responsible for filtering the pseudocoelomic fluid of the body cavity. The fluorescently-tagged peptides are taken up by bulk endocytosis (Fares and Greenwald, 2001) and analysis of their fluorescence intensity can be used as indirect measurement of DCV release. Despite the absence of DCVs from synaptic sites, secretion must still take place to some extend as VENUS-tagged neuropeptides still reach coelomocytes (Fig. VII.3).

\subsection{Active zone proteins localize to axons and dendrites in unc- 104 mutants.}

Active zone (AZ) proteins have been reported to be largely independent from UNC104 transport (Koushika et al., 2001, Shen and Bargmann, 2003, Patel et al., 2006) although the number of AZs is reduced in unc-104 mutants (Hall and Hedgecock, 1991). To compare AZ protein transport in the three different unc-104 mutants, I expressed the AZ markers RIM-1::GFP and SYD-2::GFP under the unc-129 promoter. SYD-2 and RIM-1 were exclusively expressed at presynaptic sites in wild type animals (Fig. VII. 4). As previously reported for unc-104(e1265) mutants, the localization pattern of $A Z$ proteins is more irregular and fluorescence intensity is reduced in all three unc-104 mutants compared to wild type (Sieburth et al., 2005, Yeh et al., 2005). 

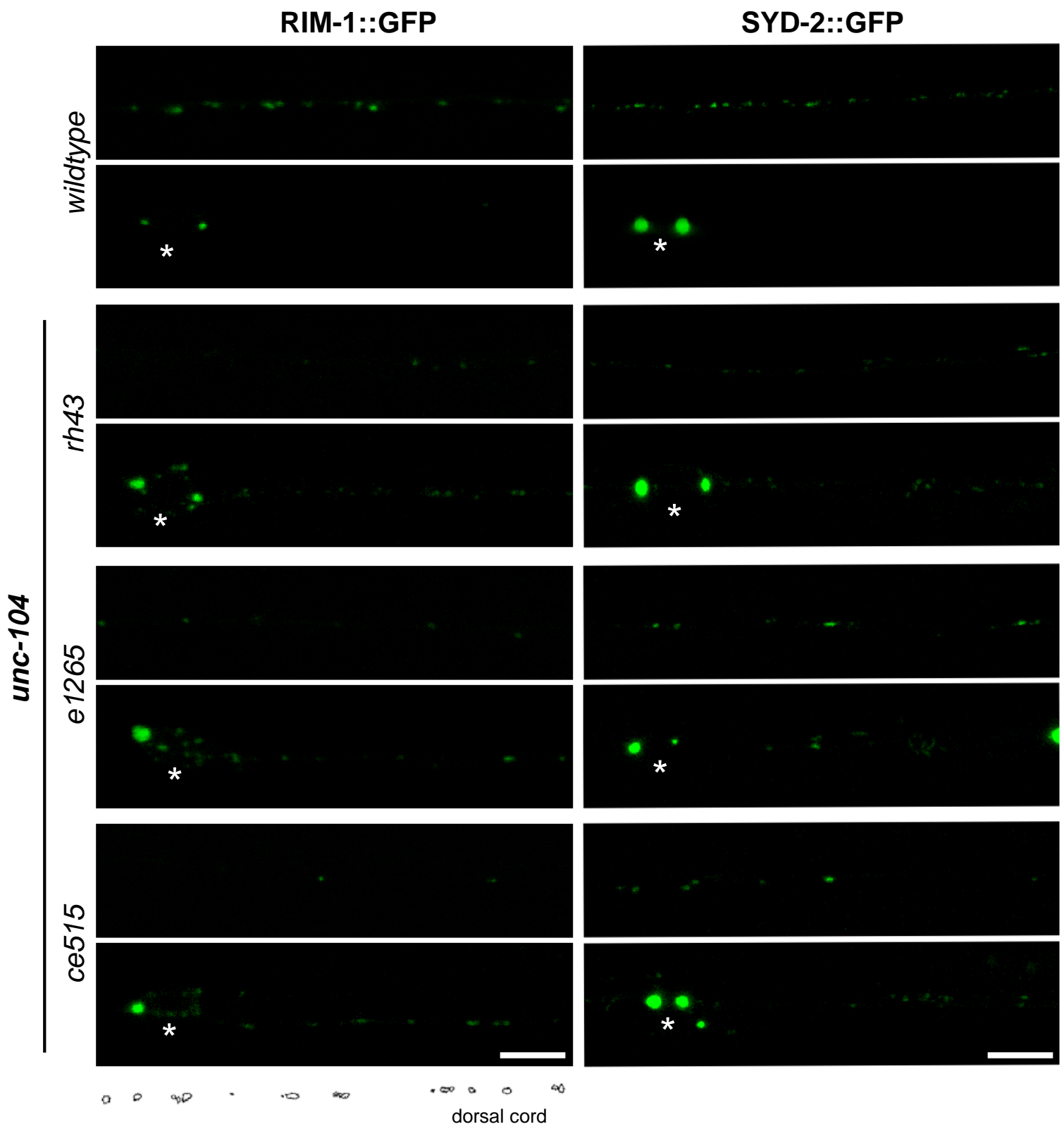

dorsal cord

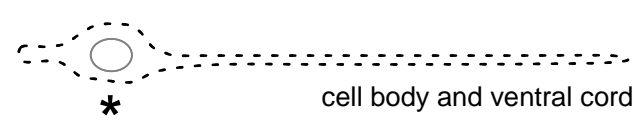

Fig. VII.4 AZ marker expression in wild type and unc-104 mutants. RIM-1::GFP and SYD-2::GFP were expressed under the unc-129 promoter. Dorsal cord (axons) and cell body with adjacent ventral cord (dendrites) are shown as indicated by scheme below. Asterisks mark position of cell body. In unc104 mutants dorsal puncta are reduced in fluorescence and number while proximal dendrites exhibit ectopically expressed RIM-1 and SYD-2. Scale bar is $5 \mu \mathrm{m}$. Crossing of the marker strains into unc104 mutant background was done by the author and Dr. Christoph Wrede. Imaging was done by the author. 
Preliminary data suggest that about $50 \%$ of the puncta in axons of unc-104 mutants are lost compared to wild type (data not shown). Instead, a fraction of the AZ proteins is transported to proximal regions of the dendrites, possibly forming ectopic AZs as suggested by the distinct localization in puncta (Fig. VII.4). These data indicate that AZ protein transport to the axon is partially maintained, but the amount of transported protein is clearly reduced when UNC-104 is defective. Retrograde transport mechanisms may prevail and thus induce transport of $A Z$ proteins into the proximal region of the dendrites.

\subsection{SVs and DCVs are absent from synapses in dorsal cord motor neurons.}

Evidence for limited transport of SV and DCV proteins to synaptic sites and reduced number of DPs has been shown above and was previously reported. Ultrastructural studies of unc-104 mutants have been performed for the nerve ring synapses and the cell bodies in the ventral ganglion (Hall and Hedgecock, 1991), but the synapse and cell body morphology of motor neurons have not yet been thoroughly investigated.

Serial section reconstruction of dorsal nerve cords in wild type and all three unc-104 mutants revealed different degrees of defective anterograde transport. In agreement with Hall and Hedgecock (Hall and Hedgecock, 1991)) some SVs and DCVs were found in the dorsal cord and usually close to a DP in unc-104(rh43) mutants despite restricted motor activity of UNC-104 (Fig. VII.5). In contrast, both alleles with disturbed cargo-binding domain (e1265 and ce515) were completely devoid of SVs and DCVs. Few and usually small DPs were found in the axons of the dorsal cord (Fig. VII.5). 

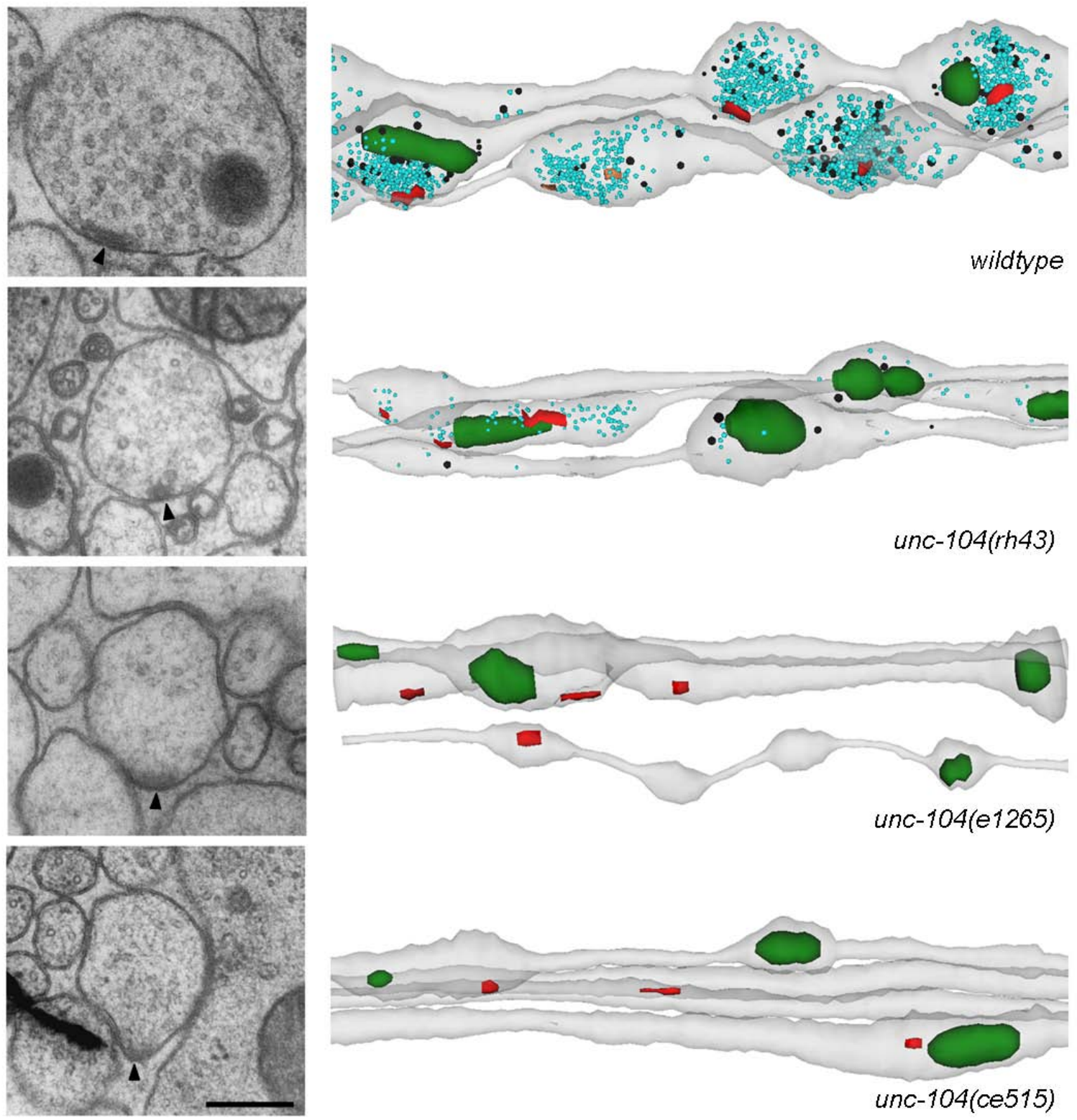

unc-104(rh43)
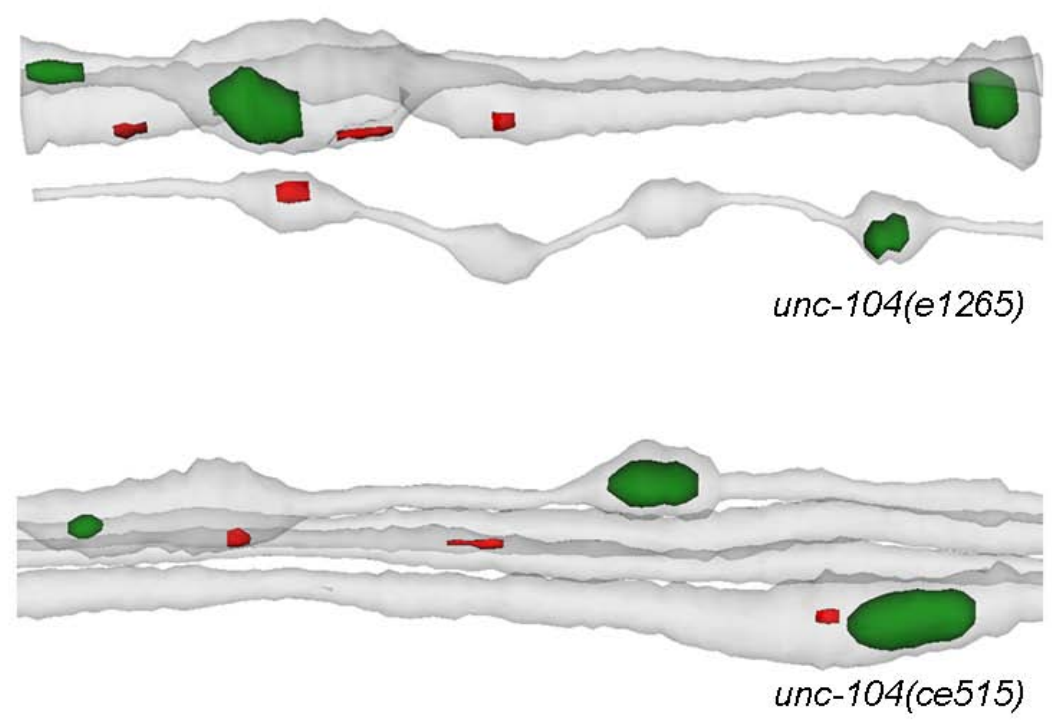

Fig. VII.5 Vesicle transport to synaptic sites is severely disturbed in unc-104 mutants. Serial section reconstructions of dorsal nerve cords reveal complete absence of SVs (light blue) and DCVs (black) in unc-104 mutants with affected PH domains (allele e1265 and ce515). Disturbed motor domains (allele rh43) lead to strongly reduced numbers of SVs and DCVs at synapses. Presynaptic dense projections (black arrowhead, red) are present in all unc-104 alleles. Scale bar is $200 \mathrm{~nm}$. For unc-104(rh43) sample preparation, imaging and alignment were done by Dr. Christoph Wrede.

Axonal swellings of the dorsal cord neurons as observed at wild type synapses are largely missing or not as prominent in unc-104 mutants. Mitochondria are normally localized in dorsal cord axons in all unc-104 strains, suggesting that other motor proteins are responsible for their transport (Hall and Hedgecock, 1991, Nangaku et al., 1994, Cho et al., 2007). 


\subsection{DCV-like vesicle accumulation and ectopic synapses formation in unc-104 mutant cell bodies}

SVs or their precursors have been reported to remain in the neuronal cell bodies of unc-104 mutants due to deficient anterograde transport (Hall and Hedgecock, 1991, Zahn et al., 2004). However, the nature of these vesicles remains unclear. Jorgensen (Jorgensen et al., 1995) showed clear core SV-like vesicles in cross sections of snt-1; unc-104 cell bodies. In contrast Hall and Hedgecock (Hall and Hedgecock, 1991) described vesicles retained in unc-104(rh43) mutant ventral ganglion cell bodies as similar to SVs but with an electron dense core. To resolve this issue, I used highpressure-freezing (HPF) and freeze substitution (FS) to prepare all three unc-104 mutant strains in a close-to-native state and analyzed the ultrastructure of motor neuron cell bodies and the enclosed vesicles. Cell bodies of all three unc-104 mutants were filled with a plethora of large DCV-like vesicles (Fig. VII.6). 


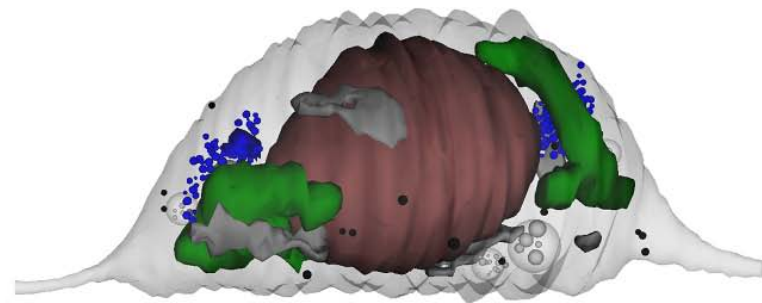

wildtype
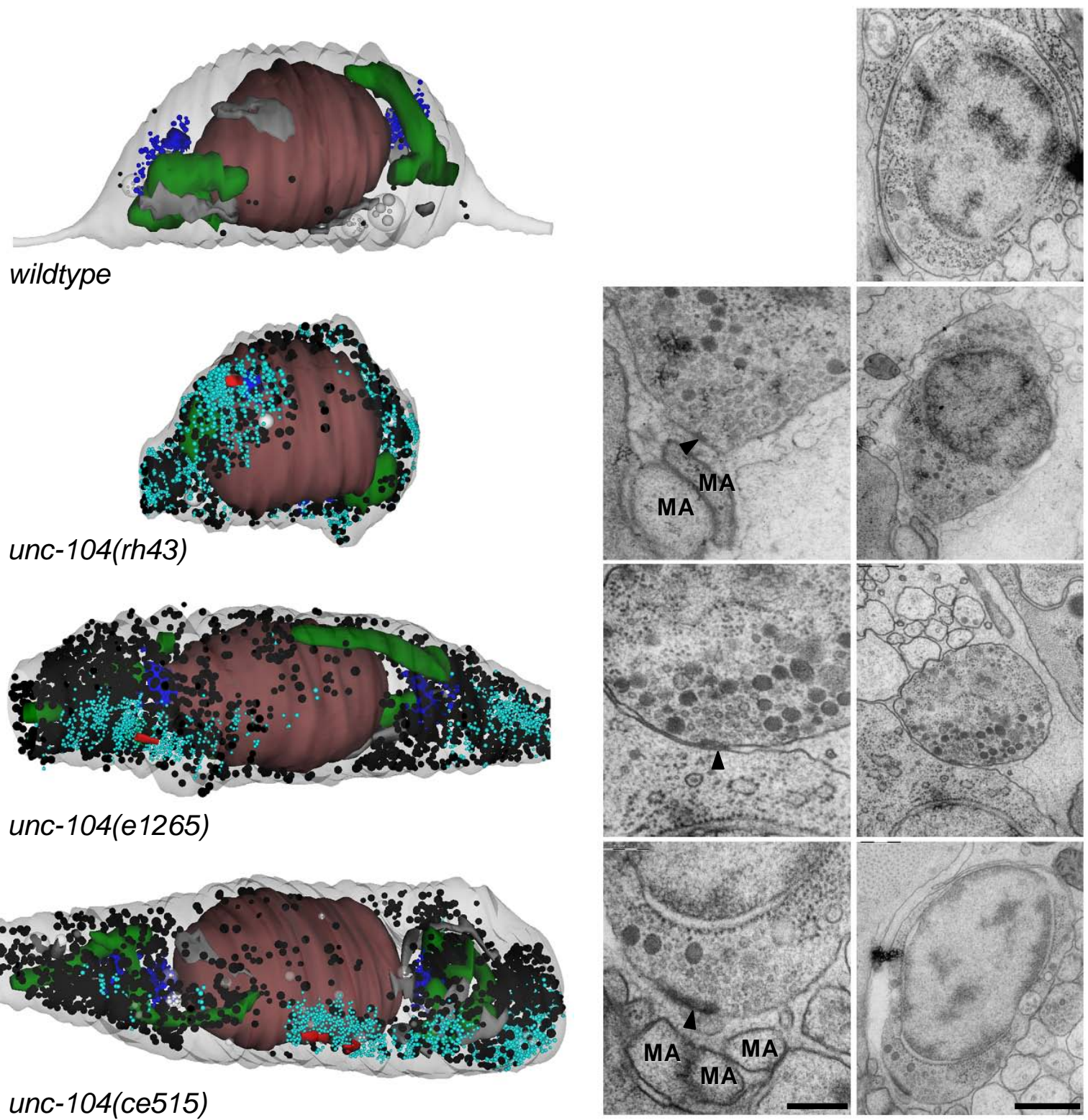

unc-104(e1265)

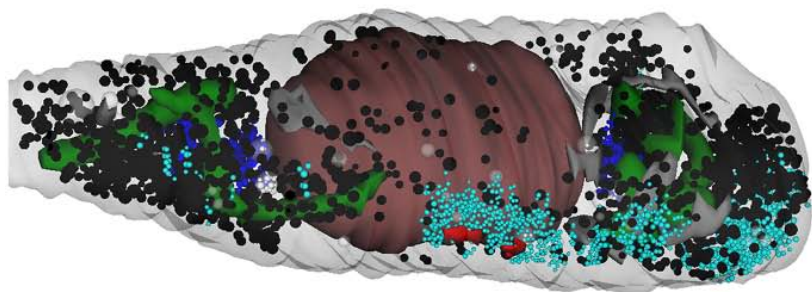

unc-104(ce515)

Fig. VII.6 Accumulation of DCV-like vesicles and ectopic synapse formation in unc-104 mutant cell bodies. Serial section reconstructions and corresponding electron microscope thin sections of cell body synapses (left) and cell body overviews (right) are shown for wild type and all three unc-104 mutants. Scale bars are $200 \mathrm{~nm}$ and $500 \mathrm{~nm}$. Cell bodies of wild type animals usually contain two Golgi complexes (blue), mitochondria (green), endoplasmatic reticulum (dark gray), multi vesicular bodies (white transparent) and the nucleus (brown). In unc-104 mutants, these organelles still localize properly. Additionally numerous large DCVs (black) fill the cell body. Some cell bodies even contain morphologically normal synapses with a DP (red, arrowheads) a SV cloud (light blue) and postsynaptic muscle arms (MA).

Quantifications revealed that unc-104 mutant alleles with stronger transport defects (e1265 and ce515) have larger DCV-like vesicles compared to rh43 and wild type (Fig. VII.7). Serial section reconstructions suggest that the excessive amount of large 
DCV-like vesicles in these two mutants causes a swelling of the cell bodies (Fig. VII.6).

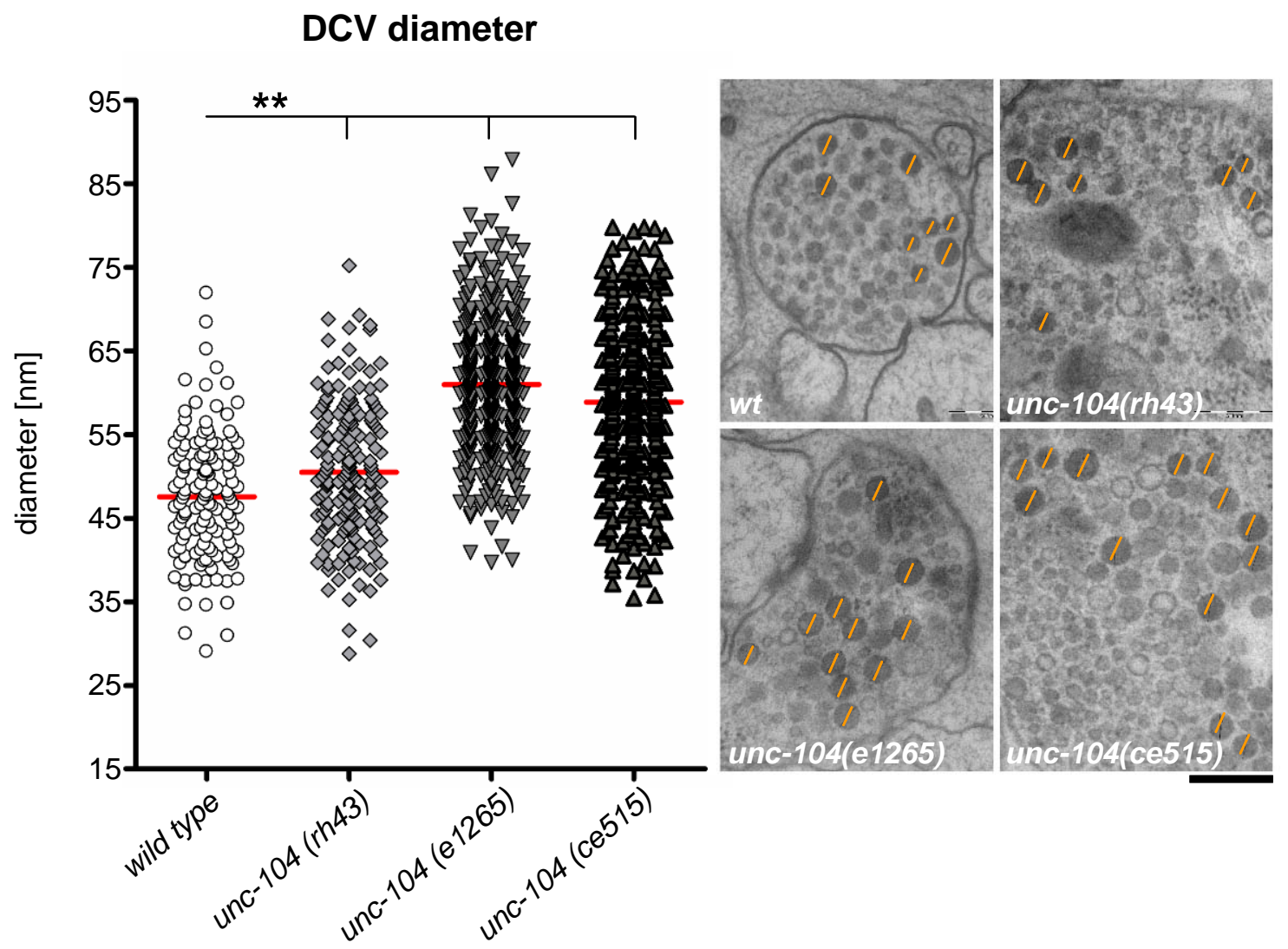

Fig. VII.7 DCV-like vesicles in the cell bodies of unc-104 mutants are larger than wild type synaptic SCVs. DCV diameters in wild type synapses were compared to DCV-like vesicles accumulating in unc-104 mutant cell bodies. Representative EM profiles are shown on the right. Vesicles considered for quantification are marked (orange line). With an average diameter of $50.5 \mathrm{~nm}$ unc-104(rh43) vesicles are larger but still rather similar to wild type DCVs $(47.5 \mathrm{~nm} ; \mathrm{p}<0.01)$. DCV-like vesicles in unc-104(e1265) and unc-104(ce515) mutants have clearly larger diameters (61.0 and 58.9 $\mathrm{nm} ; \mathrm{p}<0.01)$ compared to wild type. The mean is indicated by the red line. $\mathrm{N}$ is indicated below data points. Statistics: ANOVA and Dunnett's post-test. ( $\left.{ }^{*} \leq 0.05 ;{ }^{* *} \leq 0.01 ;{ }^{* * *} \leq 0.001\right)$. Scale bar is $200 \mathrm{~nm}$.

Occasionally, ectopic synapses were observed in motor neuron cell bodies. They exhibited a small DP and a distinct cluster of clear core vesicles. These vesicles were similar to SVs, but reduced in size (Fig. VII.8). 


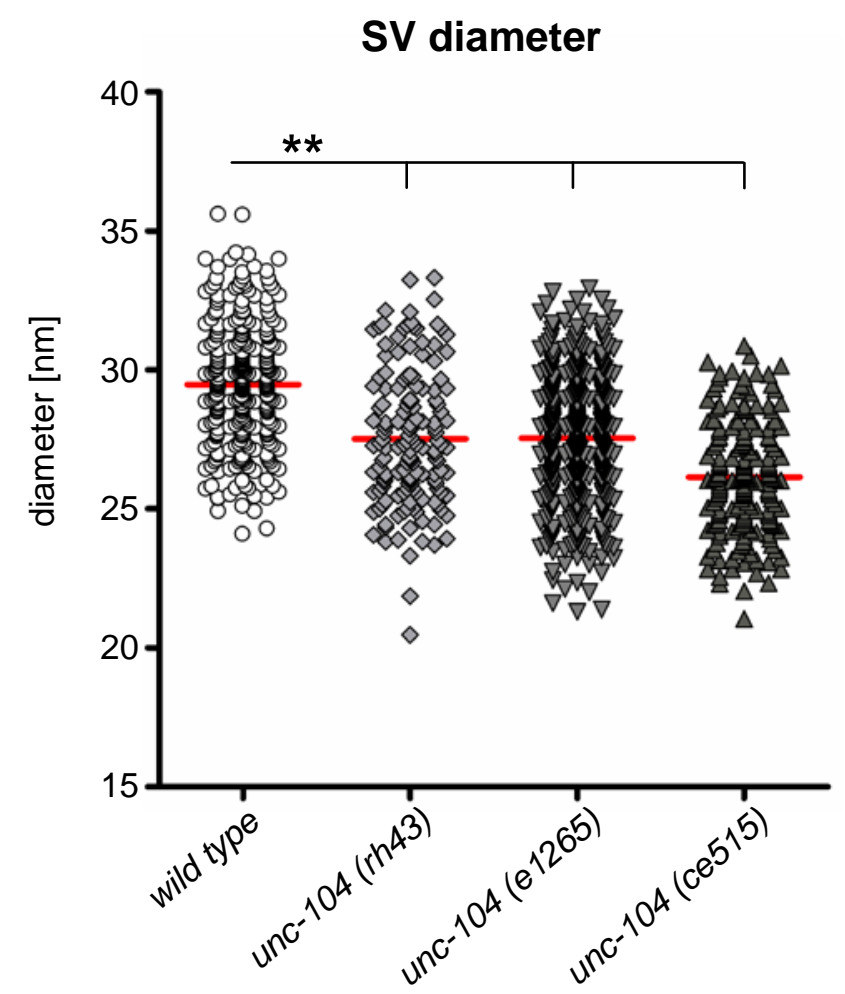

Fig. VII.8 Clear core vesicles at cell body DPs in unc-104 are smaller than wild type SVs. SV diameter in wild type synapses and unc-104 cell body synapses were quantified. Compared to wild type SVs (29.5 nm diameter), unc-104 clear core vesicles are smaller (27.5 nm in e1265 and rh43; $26.1 \mathrm{~nm}$ in ce515). The mean is indicated by the red line. $\mathrm{N}$ is indicated below data points. Statistics: ANOVA and Dunnett's post-test. $\left({ }^{*} \leq 0.05 ;{ }^{* *} \leq 0.01 ;{ }^{* * *} \leq 0.001\right)$.

Ultrastructural and fluorescence analysis demonstrated that muscle arms were usually localized directly opposite of the occasional cell body synapses, possibly representing a postsynaptic target cell (Fig. VII.6 and Fig. VII.9). 

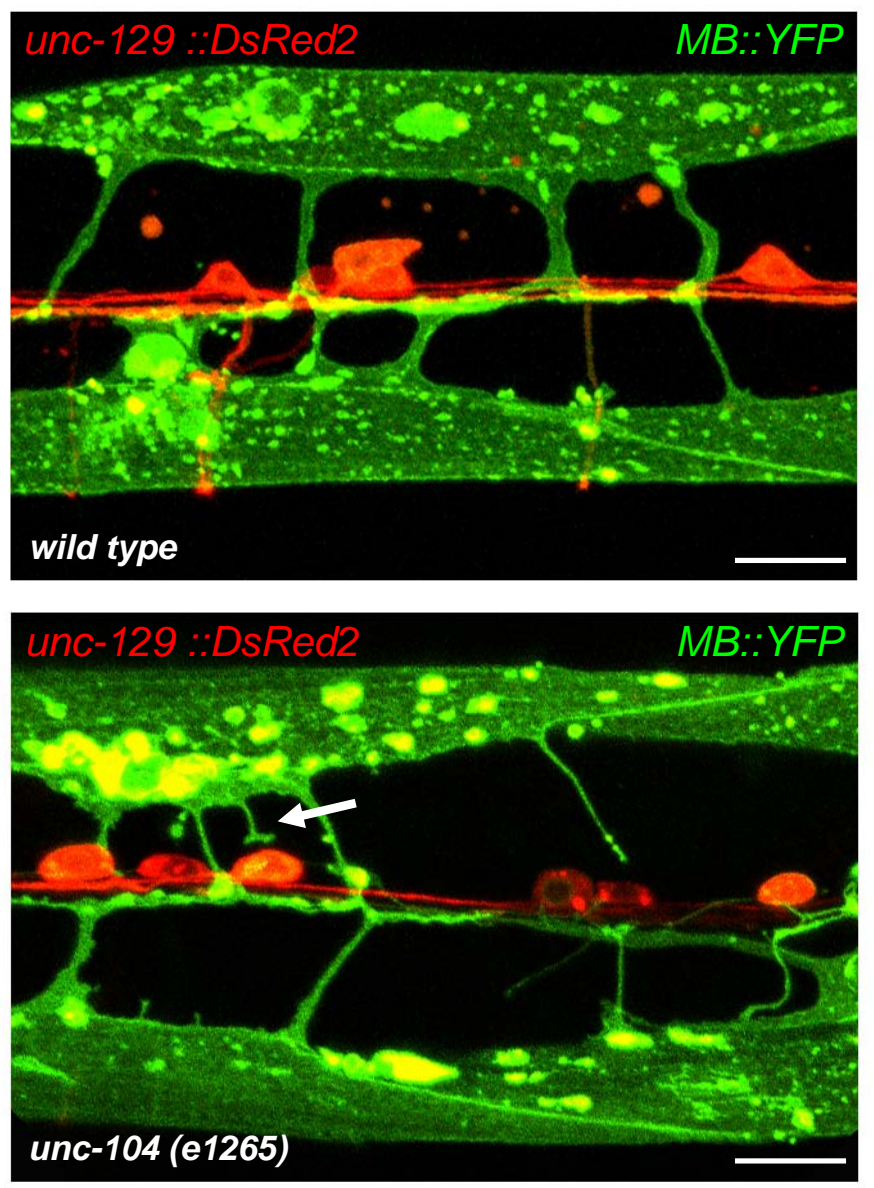

Fig. VII.9 Muscle arms contact cell bodies in unc-104 mutants. Membrane-anchored YFP (MB::YFP) was expressed in a subset of distal BWM under the him-4 promoter (green). DsRed2 was expressed in DA and DB cholinergic motor neurons under the unc-129 promoter (red). Scale bar is 10 $\mu \mathrm{m}$. In wild type animals, BWMs (asterisks) extend a distinct number of muscle arms (arrowheads) to towards axons in the adjacent (here ventral) nerve cord. In unc-104 mutants, muscle arms also contact cell bodies (arrow) and grow in a more irregular fashion.

According to my EM serial section reconstructions, other organelles like mitochondria, the Golgi complex and endoplasmatic reticulum were properly localized in the cell bodies of unc-104 mutants (Fig. VII.6) and appeared to be unaffected by impaired UNC-104 functionality.

My ultrastructural analysis revealed a unique DCV population in cell bodies of unc104 mutants. These DCV-like vesicles are larger in size compared to wild type synaptic DCVs (Fig. VII.7) and I suggest them to resemble cargo or precursor vesicles that are different from synaptic DCVs found in wild type synapses. 


\section{Discussion Chapter 2}

The involvement of the kinesin-3 family member UNC-104/KIF1A in synaptic vesicle (SV) and dense core vesicle (DCV) transport to synaptic sites had been previously suggested (Hall and Hedgecock, 1991), yet the precise implications in assembling synaptic ultrastructure and the nature of the vesicles retained in the cell bodies of unc-104/KIF1A mutants had not been determined.

I analyzed three different C. elegans unc-104 mutants. The unc-104(rh43) allele exhibits a defective motor domain whereas the other two alleles (e1265 and ce515) have a defective or truncated pleckstrin homology $(\mathrm{PH})$ domain for cargo binding, respectively.

My ultrastructural characterization via electron microscopy (EM) revealed new details about unc-104 mutant phenotypes, which had not been reported: The localization of both clear core SVs and DCVs to dorsal nerve cord axons of motor neurons is completely abolished when lipid-binding via the PH domain is impaired (alleles e1265 and ce515) (Fig. VII.5). However, the formation of dense projections (DPs) at presynaptic sites is largely unaffected by either defective UNC-104 PH or motor domains. Lesions in the motor domain in unc-104(rh43) compromise but do not abolish vesicle transport. Reduced numbers of both SVs and DCVs can be found close to DP in the dorsal cord axons of these mutants.

Second, vesicles with a dense core are the predominant class of vesicles accumulating in the cell bodies of all three unc-104 mutants analyzed. These DCVlike vesicles are significantly larger than the DCVs typically found in wild type presynaptic terminals containing neuropeptides. The increased size of these DCVlike vesicles is more pronounced in mutants with defective $\mathrm{PH}$ domain than mutants with defective motor domain (Fig. VII.7).

Third, synapses were occasionally formed in cell bodies of all three unc-104 mutants analyzed. The morphology of these synapses was wild type-like in the way that SVs clustered around the DP and postsynaptic muscle arms localized opposite to the synapse (Fig. VII.6).

These ultrastructural findings were supported by analysis of fluorescently-tagged marker proteins for SVs, DCVs and active zones (AZs). In unc-104 mutants, SV and DCV marker fluorescence was completely absent or below detection level in dorsal nerve cord axons, suggesting that no or only very few SV and DCV proteins were 
transported in anterograde direction. However, a clear increase of RAB-3::VENUS fluorescence was observed in the cell bodies of these mutants. In line with the ultrastructural observations, this finding suggests that SV and DCV proteins are retained in the cell body due to compromised UNC-104 transport.

In contrast, fluorescence derived AZ markers RIM-1 and SYD-2 were still observed in distinct puncta along the nerve cord, resembling the position of presynaptic terminals. However, fluorescence intensity was reduced and additional puncta appeared in the ventral cord dendrites of the same motor neurons. These findings revealed new insights into the role of UNC-104/KIF1A in synaptogenesis.

\subsection{UNC-104 specifically transports large DCV-like SV precursors}

The cell bodies of the unc-104 mutants analyzed in this study were filled with a plethora of DCV-like vesicles from 35 to $85 \mathrm{~nm}$. In contrast, only very few clear core SVs or SV-like vesicles were found in the cell body, except for rare ectopic synaptic sites. At the same time, dorsal cord axons of mutants with defective $\mathrm{PH}$ domain were completely devoid of SVs or synaptic DCVs. These findings suggest two things: UNC-104 transports large DCV-like vesicles and these DCVs are likely to be SV precursors. In line with this hypothesis, the DCV-like vesicles are morphologically different from synaptic DCVs in presynaptic terminals of wild type animals, indicating that they belong to a different class of DCVs. The electron dense core indicates a proteinaceous content. In line with their proposed function as SV precursor, they should contain SV proteins but not neuropeptides. Labeling with antibodies against specific SV and DCV proteins (e.g. Rab-3 and NLP-21, respectively) via immuno-EM would be one step to identify the specific content and function of these DCV-like vesicles. Once a component specific for these DCV-like vesicles is known, immunoisolation of these vesicles from unc-104 mutants could be performed similar to previously reported experiments in rat brain (Zhai et al., 2001, Shapira et al., 2003). Subsequent SDS-PAGE and Western blot analysis of coimmuno-isolated proteins could identify further components of the DCV-like vesicles. On the basis of the protein content, the SV precursor hypothesis could be refined or rejected.

Fluorescence analysis of synaptic protein markers in the dorsal and ventral nerve cord synapses of $C$. elegans allowed me to do a quick initial examination of defects in 
SV and DCV transport and localization. However, like many other transgenic markers, these fluorescently-tagged synaptic proteins are overexpressed and exhibit some fluorescence in the wild type cell bodies. Therefore, their suitability to resolve accumulation of fluorescently-tagged proteins in the cell bodies is limited. Fortunately, the cell body accumulation of RAB-3::VENUS was very low in wild type animals. Hence, this construct could be used in unc-104 mutants to clearly observe the increase in ectopic RAB-3::VENUS signal in the cell bodies. This accumulation was pronounced around the two Golgi complexes on both sides of the nucleus where DCVs are generated (Fig. VII.2). In the absence of SVs in the cell body, this indicates that RAB-3 might be localized on the DCV-like vesicles instead, supporting my hypothesis that they might be SV precursors. The accumulation of fluorescentlytagged neuropeptides NLP-21 and INS-22 suggests that UNC-104 transports DCVlike vesicles as well as synaptic DCVs, possibly via similar interactions or mechanisms.

The observation that the DCV-like vesicles are smaller in unc-104 mutants with defective motor domain (allele rh43) than in mutants with defective cargo-binding domain (alleles e1265 and ce515) (Fig. VII.7) could be explained by a feedback mechanism after initial release at the synapses in unc-104(rh43) motor neuron axons. In fact, feedback mechanisms after DCV fusion via IDA-1 have been proposed for DCV biogenesis (Cai et al., 2009). In unc-104(rh43) mutants moderate amounts of SVs and synaptic DCVs were observed via electron microscopy (EM) around DPs in presynaptic terminals (Fig. VII.5). I assume that release can take place at these synapses, as individual AZ DPs seem morphologically normal. After neurotransmitter release, an intracellular or extracellular signaling cascade could provide feedback to the cell body where the generation of SV precursors, possibly the observed DCV-like vesicles, is down-regulated. Hence the number of DCV-like vesicles would decrease in unc-104(rh43) mutants. Smaller size synaptic DCVs might, however, be continuously generated and largely remain in the cell body due to compromised UNC-104 function. This would result in the accumulation of smaller synaptic DCVs rather than larger DCV-like SV precursors in unc-104(rh43) mutants.

This feedback mechanism would be abolished in unc-104(e1265) and unc104(ce515) mutants due to the complete absence of SVs at presynaptic terminals and, consequently, lack of synaptic transmission. Antibody labeling of neuropeptides 
like NLP-21 or INS-22 via immuno-EM could be a way to differentiate the DCV-like vesicles from the neuropeptide-containing synaptic DCVs.

\subsection{Active zone components are transported differently than SV precursors and DCVs}

Despite the transport defects in all three unc-104 mutants analyzed in this study, the formation of DPs in dorsal nerve cord motor neuron axons was only moderately affected. This indicates that the transport of $A Z$ material is independent of $S V$ precursor and DCV transport. This argument is in line with the hypothesis that $A Z$ proteins are associated with a different organelle than SV and DCV proteins as previously suggested by Okada and colleagues (Okada et al., 1995). They showed that vesicles associated with KIF1A contained SV proteins such as synaptotagmin, synatophysin, and Rab3A but not AZ proteins like syntaxin 1A or SNAP25 (Okada et al., 1995). They additionally identified $80 \mathrm{~nm}$ DCVs, which associated almost exclusively with AZ components but not with SV proteins in mammalian brain lysates (Zhai et al., 2001, Shapira et al., 2003). Although the nature of AZ transport organelles remains unclear, these finding suggest that they might resemble a third class of DCVs. Evidence from photoreceptor ribbon synapses in the mouse retina supports this notion. Ribbon-associated proteins Piccolo, Bassoon, RIBEYE and RIM1 were shown to be transported in electron dense precursor spheres to nascent presynaptic sites early in synaptogenesis (Regus-Leidig et al., 2009). With the postnatal formation of mature synaptic ribbons, the number of precursor spheres declined.

In line with previous observations in the C. elegans nerve ring (Hall and Hedgecock, 1991), I found that the number of DPs is moderately reduced in unc-104 mutant motor neurons. During the collection of serial section electron micrographs I had the impression that the frequency of DPs in dorsal cord axons is lower in the two mutant alleles e1265 and ce515. They both have a stronger transport defect compared to unc-104(rh43) such that no vesicles reach the dorsal nerve cord. Of these two strains, unc-104(ce515) had the lowest frequency of DPs and exhibited significantly stronger uncoordinated (unc) movement. Adult worm body size of unc-104(ce515) mutants is reduced compared to unc-104(e1265) or wild type animals. It is 
conceivable that the complete deletion of the $\mathrm{PH}$ domain in allele ce515 affects additional activities of this domain, besides binding to $\mathrm{PI}(4,5) \mathrm{P}_{2}$ in vesicle membranes. In fact, Kumar and colleagues showed that the UNC-104 PH domain preferentially binds to $\mathrm{PI}(4,5) \mathrm{P}_{2}, \quad \mathrm{PI}(4) \mathrm{P}$ and brain lipids like Phosphatidylethanolamine, Phosphatidylserine, Phosphatidic acid and others. It can additionally bind phosphatidylcholine (PC) and phosphatidylinositol (PI). The lesion in the unc-104(e1265) $\mathrm{PH}$ domain leads to a reduced affinity to $\mathrm{PI}(4,5) \mathrm{P}_{2}, \mathrm{PI}(4) \mathrm{P}$ and brain lipids. However, PC and PI are still bound (Kumar et al., 2010). A complete loss of the PH domain as in unc-104(ce515) may therefore lead to an overall abolished binding to lipids and thus result in a stronger phenotype than unc-104(e1265).

Thorough quantification of fluorescently-tagged AZ markers would allow quantification of DP frequency over the whole length of motor neuron axons and might support or refute my initial impression. First confocal images the number of fluorescent puncta from AZ proteins SYD-2::GFP and RIM-1::GFP in the dorsal nerve cords did not show a clear difference between the three unc-104 mutants (Fig. VII.4). If the number of DPs correlates with the degree of defective transport, one interpretation would be that AZ proteins are transported via UNC-104 or at least in collaboration with UNC-104. This transport should occur in organelles that bind differently to UNC-104 than DCV-like vesicles or synaptic DCVs. The AZ protein Liprin-a/SYD-2 has been shown to interact with the stalk region of KIF1A/UNC-104 in vitro and in vivo (Shin et al., 2003, Wagner et al., 2009). It is conceivable that motor proteins use SYD-2 to identify $A Z$ transport organelles and that the Liprin-binding domain of UNC-104/KIF1A mediates this interaction. The point mutations in the unc104 mutants analyzed in this study should not affect the stalk region with the putative Liprin-binding domain. Taking into account the mutation sites of the three unc-104 mutants analyzed, I assume that the binding site for SYD-2 is intact and AZ protein transport can still be accomplished. The reduced number of DPs could then be accredited to the reduced levels of UNC-104 as it was reported for mutants with defective cargo binding in vivo (Kumar et al., 2010). As already discussed in paragraph V., unc-104 mutants with defective Liprin-binding domain are not available for $C$. elegans and would have to be generated by chance via methods like Ethyl methanesulfonate (EMS) mutagenesis. However, it is not clear whether these mutants would have a visible phenotype to allow selection in a mutagenesis screen. 
Apart from that, in vitro studies could provide valuable insight into SYD-2 interaction with UNC-104.

Other explanations for the correlation between reduced DP number and defective transport in unc-104 mutants are imaginable: Muscle arms from dorsal body wall muscles have been shown to be misguided to the ventral nerve cord and to cell bodies in unc-104(rh43) and unc-104(e1265) mutants (Hall and Hedgecock, 1991; this work). However, the contact to postsynaptic muscles cell was proposed to be necessary for successful synaptogenesis in $C$. elegans motor neurons (Plunkett et al., 1996). Formation of AZs in the dorsal nerve cord axons may therefore be compromised due to reduced muscle-neuron interaction in unc-104 mutants.

Also, impaired transport of proteins specifically involved in the formation and maintenance of the complex AZ network could be disturbed in unc-104 mutants. For instance, clustering of postsynaptic AMPA receptors depends on the UNC-104 binding partner SYD-2/Liprin-a (Wyszynski et al., 2002). Mislocalization of SYD-2 and its downstream targets might cause impaired synapse formation, maturation and stabilization. There is evidence from other organisms that specific proteins are needed to ensure synapse formation and maintenance (reviewed in Garner et al., 2002). Nerve-derived Agrin was found to be crucial for synapse growth and maintenance at vertebrate skeletal neuromuscular junctions (Lin et al., 2001), whereas the cell-adhesion molecule integrin is required for maturation of hippocampal synapses (Chavis and Westbrook, 2001).

The lack of transmission activity could additionally lead to the elimination of $A Z s$ (Verhage et al., 2000). Disassembly of AZs could be mediated by RSY-1, which has been proposed to eliminate ectopic synapses in HSN axons during development (Patel and Shen, 2009). The regulation of Liprin- $\alpha$ /SYD-2 levels via $\mathrm{Ca}^{2+}$-calmodulindependent kinase II (CaMKII) (Hoogenraad et al., 2007) and APC/C complex (van Roessel et al., 2004) could be another mechanism to facilitate $A Z$ removal. As discussed in paragraph $\mathrm{V}$, loss of Liprin-a/SYD-2 leads to strongly reduced DP size and possibly loss of AZs.

Further investigations are necessary to understand which kinesin or other motor proteins are responsible for $A Z$ component transport, which adaptor proteins and transport organelles are involved and how $A Z$ cargo unloading is accomplished at nascent presynaptic sites. 


\subsection{Ectopic synapses are formed in unc-104 mutant cell bodies}

In all three analyzed unc-104 mutants, we occasionally observed cell bodies with SV clusters around a small DP. These ectopic synapses were never found in wild type animals. Formation of synapses in unc-104 mutant cell bodies is likely due to the local accumulation of synaptic proteins.

In general, formation of AZs could be triggered by initiation of signaling cascades upon contact of neurons with postsynaptic target cells (Hazuka et al., 1999, Ahmari et al., 2000, Bruses, 2000). Subsequent cytoskeleton remodeling could prepare synaptic sites for AZ and DP formation and recruit SV precursors. For instance Drosophila Dock, a SH2-SH3 domain adapter protein, was suggested to play a role in remodeling the growth cone cytoskeleton in developing axons during formation of mature NMJ presynaptic terminals (Desai et al., 1999). AZ transport organelles like the proposed subclass of the large DCV-like vesicles could be transported to nascent synaptic sites. By fusing with the plasma membrane they could deposite their cargo and initiate the formation of the $A Z$ scaffold. Subsequently, recruitment of SV precursors to these AZs could be initiated. To date it is unclear how and where SVs are generated. One possibility is that SV precursors fuse with the presynaptic plasma membrane. Internal sorting and organization of SV proteins and subsequent endocytosis could provide a mechanism for local SV formation de novo (reviewed in Hannah et al., 1999).

The fluorescence analysis in this study showed that a multitude of precursors containing SV or AZ proteins, respectively, accumulate in unc-104 mutant cell bodies. As suggested previously (Hedgecock et al., 1990, Hall and Hedgecock, 1991), motor axon terminals might release diffusible factors to attract muscle arms. These factors might be released from vesicles accumulating in unc-104 mutant cell bodies, triggering postsynaptic muscle arms to grow towards the cell bodies rather than the nerve cord. Contact with a postsynaptic partner may then induce synapse formation in the cell body in a similar manner as in wild type motor axons (Plunkett et al., 1996). I propose that the ectopic synapses observed in unc-104 mutant cell bodies are likely to be functional as postsynaptic muscle arms localize directly opposite to the $A Z$. Moreover, SVs cluster around a DP, suggesting that the machinery for vesicle fusion as well as retrieval has been established. The release of fluorescently-tagged 
neuropeptides NLP-21::GFP and INS-22::GFP into the body cavity was indirectly observed, but not yet quantified, by its endocytic accumulation in coelomocytes. These cells are macrophage-like and responsible for filtering the pseudocoelomic fluid of the body cavity in C. elegans. Although DCVs probably fuse outside the AZ to release their neuropeptides, this process requires a regulatory fusion machinery typically found at synapses (Martin and Kowalchyk, 1997).

\subsection{Perspective}

To gain more insight into the individual steps of synapse formation, I will take advantage of the "empty" state of dorsal motor neuron axons in unc-104(e1265) and unc-104(ce515) mutants. A heat shock construct to inject and express wild type UNC-104 in the unc-104 mutant background is currently generated. At the permissive temperature, UNC-104 protein will be expressed specifically in C. elegans motor neurons and should restore synapse morphology.

Confocal microscopy analysis of SV and DCV marker protein localization and corresponding high-pressure-freeze EM can be used to capture different time points during synaptogenesis after induction of UNC-104 expression. This might allow me to follow the individual steps to synapse formation, specifically the formation of the $A Z$ and the SV pool. If my hypothesis is true that large DCV-like vesicles resemble SV precursors, they should first arrive at AZs and mature SVs should only appear later in the presynaptic terminal but not in synapse free regions of the axon as the commissures. It will also be interesting to see whether ectopic synapses in the cell bodies are preserved in mutants upon wild type UNC-104 expression. If muscle arms are retracted to be innervated by their adjacent nerve cord, it would indicate a correlation between DCV precursor localization and muscle arm guidance as proposed by Hall and Hedgecock (Hedgecock et al., 1990, Hall and Hedgecock, 1991).

As motor protein involved in the transport of SV precursors and possibly AZ material, UNC-104/KIF1A function is of major interest in the research field of synaptogenesis. The viability of mutants and the ability of hermaphrodites to reproduce despite strong phenotypes make $C$. elegans a great model organism to further study the role and 
Discussion Chapter 2

regulation of kinesins in general and UNC-104 specifically to elucidate their implication in synapse formation. 


\section{Materials and Methods}

\subsection{Strains}

Strains were grown at $20^{\circ} \mathrm{C}$ as described (Brenner, 1974). Strains used in this study are listed in Table 1.

Table 1 C. elegans strains and source used in this study

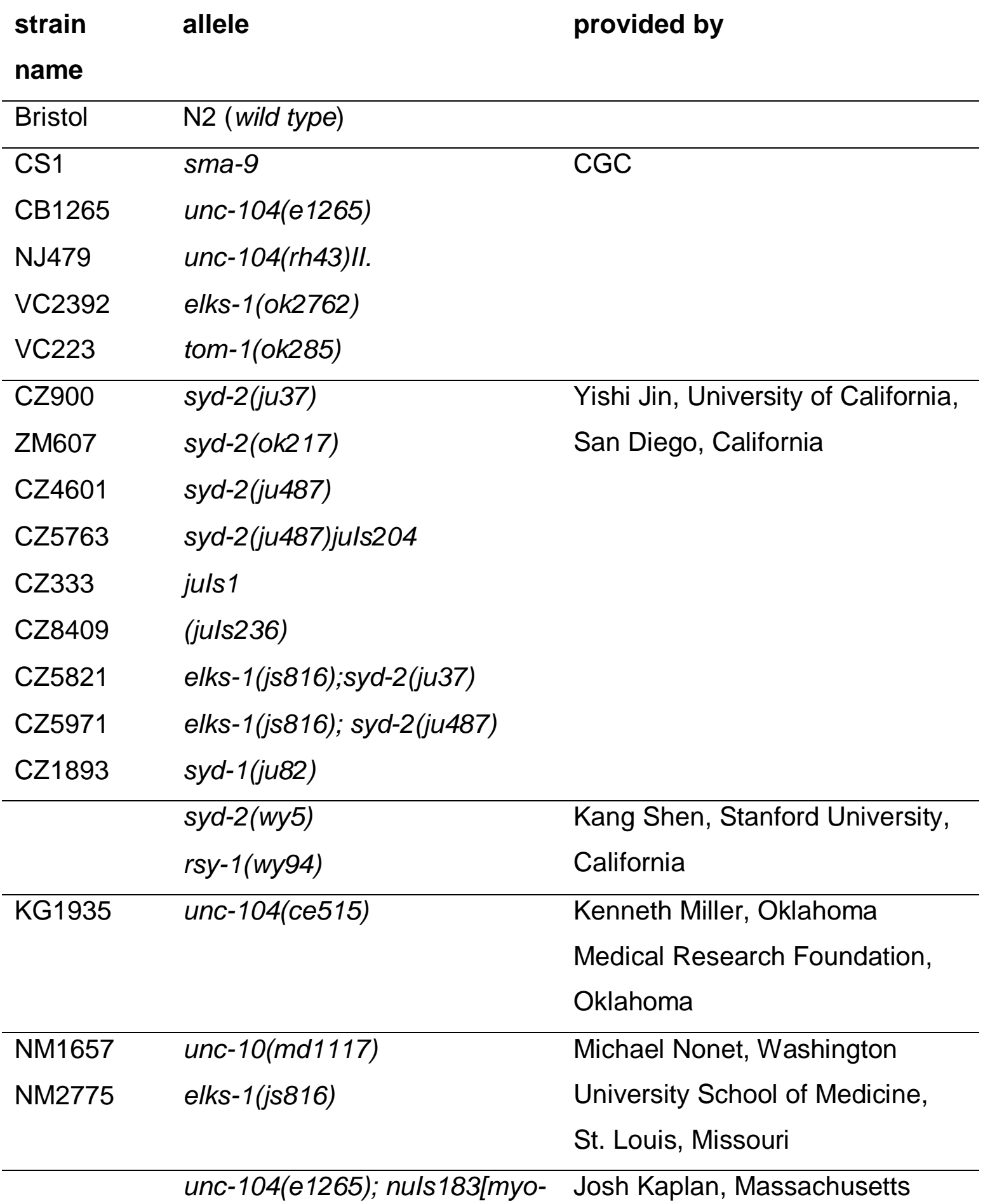




\begin{tabular}{lll}
\hline & $2:: g f p ;$ unc-129::nlp-21-venus] & General Hospital, Boston, \\
& III & Massachusetts \\
KP3292 & nuls152[ttx-3::mRFP; punc- & \\
& 129::gfp-snb-1] & \\
KP3931 & nuls168[myo-2::gfp; punc- & \\
& 129::venus-rab-3] & \\
\hline GQ584 & nuls183[punc-129::nlp-21- & Original strain provided by Josh \\
& venus] & Kaplan, Massachusetts General \\
GQ585 & nuls195[myo-2::gfp; punc- & Hospital, Boston, MA; out- \\
& 129::ins-22-venus] & crossing of marker allele in the \\
GQ179 & nuls159[ttx-3::mRFP; punc- & Eimer lab \\
& 129::gfp-syd-2] & \\
GQ178 & nuls165 [myo-2::gfp; punc- \\
& 129::unc-10-gfp] & \\
trls30 (him-4p::MB::YFP + \\
\end{tabular}

\subsection{Genetics}

Primer sequences listed on wormbase (http://www.wormbase.org/species/c elegans/ variation/WBVar00091522\#02-45-3) were used to verify deletion in syd-2(ok217) mutants. All other syd-2 mutant strains were sequenced with primers listed in the Appendix Table 5, generated with ApE (Paradis et al., 2004). Due to mild or no phenotypes in syd-2 mutants, crossed were performed over the balancer sma- 9 . Males from strains carrying fluorescently-tagged marker proteins (CZ333, CZ8409, nuls152, nuls159, nuls165, nuls168; nuls183; nuls195; trls30) were crossed with hermaphrodites of syd-2;+/sma-9;+ or unc-104 mutants. Syd-2 homozygotes were verified by sequencing or PCR; unc-104 mutants by unc-phenotype.

The syd-2(ju487) overexpression mutant was generated by Ya Dai as follows: The extrachromosomal array (juEx1011) was made of 20ng/ul syd-2(ju487) full length genomic PCR product amplified using primers YJ3748 (ggtgaagattgcgattcgg) and YJ3727 (atgcacgcatagttagaacgg), coinjected with Pttx-3-RFP. The mutant was generated by UV-trimethylpsoralen mutagenesis of the strain: juEx1011; syd- 
2(ju487)X. The integrant was backcrossed against N2, and found to be linked to the X chromosome, near syd-2.

\subsection{High-pressure-freezing / Freeze substitution}

A aluminium platelet of $2 \mathrm{~mm} \varnothing$ and $100 \mu \mathrm{m}$ depth (Microscopy Services, Flintbek) was filled with a thick suspension of E. coli OP50 and water. 20 to 30 young adult worms were transferred into the chamber and cryofixed using a BalTec HPM10. Freeze substitution was carried out in a Leica EM AFS according to (Rostaing et al., 2006). Briefly, worms were incubated in $0,1 \%$ tannic acid at $-90{ }^{\circ} \mathrm{C}$ for $100 \mathrm{~h}$, then washed and incubated for $32 \mathrm{~h}$ in $2 \% \mathrm{OsO}_{4}$ (each w/v in dry acetone), slowly increasing temperature to $4^{\circ} \mathrm{C}$. Infiltration with Epoxy resin (Agar Scientific, AGAR 100 Premix kit hard) was done at room temperature with increasing concentration from $50 \%, 90 \%$ to $100 \%$ over $48 \mathrm{~h}$. Finally worms were embedded in Epon between two glass slides, one covered with a layer of Teflon spray. A stripe of parafilm (Pechiney Plastic Packaging Company) on each short side of the slide was used as space holder.

\subsection{Production of formvar coated grids}

A $0.5 \%$ Formvar (Polyvinylformaldehyd; PLANO) solution was made in water-free chloroform. A dropping funnel was filled about $3 \mathrm{~cm}$ high with the formvar solution so that a clean glass slide was covered to $2 / 3$. The solution was drained from the funnel leaving a thin layer of formvar on the glass slide. The slides were set aside to dry for at least 10 minutes. To detach the formvar film from the slide, it was cut with a blade along the rim of the slide and dipped slow and perpendicularly into a 2 liter beaker filled with water. Formvar film will float off and detach from the glass slide. Grids were placed onto the films and picked off with a stripe of parafilm (Pechiney Plastic Packaging Company).

\subsection{Electron microscopy}

$50 \mathrm{~nm}$ thin sections or $250 \mathrm{~nm}$ thick sections for tomography of C. elegans were cut with an Leica UC6 ultramicrotome. Ribbons of consecutive sections were transferred 
onto Formvar-coated copper slot-grids (PLANO; G2500C) or 200 Mesh grids (PLANO; G2200C). For post-staining, grids were incubated for $45 \mathrm{~min}$ on drops of 4 $\%(w / v)$ uranyl acetate in water and then washed in distilled water. After removing excessive water with filter paper (Macherey-Nagel), grids were placed on drops of lead citrate (Reynolds, 1963) for $2 \mathrm{~min}$ in a $\mathrm{CO}_{2}$-free chamber (petri dish with wet $\mathrm{NaOH}$ pellets; Roth) and rinsed in distilled water. Excessive water was removed with filter paper. Micrographs were taken at a Zeiss EM 902A, operated in the bright field mode at $80 \mathrm{kV}$ with a $1024 \times 1024 \mathrm{CCD}$ (charge-coupled divice) detector (Proscan CCD HSS 512/1024; Proscan Electronic Systems, Scheuring, Germany). GABAergic and cholinergic neurons were discriminated by their postsynaptic partners (Hallam et al., 2002).

\subsection{Serial section reconstruction}

Micrographs of consecutive sections were imported into "Reconstruct" (Fiala, 2005) and aligned in the rigid mode. Vesicles and multivesicular bodies were marked in the center of the structure with the circle tool encoding their maximal diameter and later displayed as sphere. All larger components (DP, membranes, nucleus, mitochondria, ER) were traced in all sections and displayed as "Boissonnat surface" with maximal facets. For large structures (membranes, nucleus, mitochondria) z-traces were generated through the center of the structure and smoothened with a Moving Average Filter Length of 10.

\subsection{Electron tomography}

250 and $350 \mathrm{~nm}$ post-stained cross sections (see 9.5). Cationic gold tracers (15nm, EM Grade; Aurion Cat\# 25449) were applied for 5 minutes on both sides of the grid and later used as fiducial markers for volume reconstruction.

Carbon coating was applied on both sides. Two tilt series shifted $90^{\circ}$ against each other were recorded at a JEOL 4000EX TEM with a 4Kx4K lens coupled CCD camera at $400 \mathrm{KV}$ and $25000 \mathrm{x}$ magnification or a JEOL2100 TEM with a $4 \mathrm{~K} \times 2.6 \mathrm{~K}$ Orius SC1000A camera. Each series was collected from $-60^{\circ}$ to $60^{\circ}$ with 2 degree increment. 
To minimize specimen deformation during the image collection process, irradiation at all tilt angles was done before collecting the tilt series. Alignment and reconstruction of the two tilt series was done using IMOD software package (Kremer et al., 1996). For tomograms of wild type and syd-2 mutants, fine alignment and reconstruction was done using TxBR package (Lawrence et al., 2006). DP volume segmentation was done by manual tracing using the magic wand tool in Amira software (http://www.amira.com).

\subsection{Quantification of EM data}

Analysis of Electron micrographs was done using imageJ (http://rsbweb.nih.gov/ij/index.html). Vesicle diameters were analyzed with the "straight line" tool. The distance of docked vesicles to the dense projections was measured with the "segmented line" tool from the midpoint of the vesicle/membrane contact along the presynaptic membrane to the closest DP/membrane contact. Bouton size of the synapses was determined with the "polygon selection" tool.

\subsection{Fluorescence microscopy and quantification}

Fluorescence patterns were analyzed via confocal light microscopy. Live animals were placed in on $2 \%$ agarose pads with $50 \mathrm{mM} \mathrm{Na-azide} \mathrm{in} \mathrm{M9} \mathrm{buffer} \mathrm{for}$ immobilization. Images were obtained on an inverted confocal microscope (SP2; Leica) with a $63 \times$ NA 1.32 oil immersion objective at $20^{\circ} \mathrm{C}$. Image stacks of the nervecords and cell bodies were collected and average and maximum intensity projections calculated using Leica confocal software. The posterior part of the dorsal nerve cord was imaged in young adult animals when oriented toward the objective. For quantification maximum intensity projections obtained with the same settings were loaded into ImageJ (http://rsbweb.nih.gov/ij/index.html). Projections were transformed to 16-bit images, the same threshold was set and total fluorescence analyzed using the "polygon selection" tool. 


\subsection{Western blot}

For SYD-2 protein quantification mixed staged worm extracts were prepared (modified after Gally et al., 2004). Six large plates $(\phi 9 \mathrm{~cm}$ ) of worms were rinsed off and washed two times with M9 buffer, pelleted by centrifugation and resuspended in $400 \mu \mathrm{l}$ of homogenisation buffer (see 11.2) including protease inhibitor (Roche cOmplete, EDTA-free Protease Inhibitor Cocktail Tablets). Samples were frozen at $80^{\circ} \mathrm{C}$ for 30 minutes, sonificated 3 times $30 \mathrm{sec}$ and total protein content was measured with a Nanodrop (PEQLAB ND-1000). Wormlysis was mixed 1:1 with 2xSDS sample buffer (see 11.2) and denatured for 10 minutes at $90{ }^{\circ} \mathrm{C}$. Equal amounts of total protein extract per strain were loaded onto a $7.5 \%$ stacking gel run at $40 \mathrm{~mA}$ and resolved on a separating SDS-polyacrylamide gel (see 11.2) run at 80 $\mathrm{mA}$ in $1 \mathrm{x}$ SDS running buffer (see 11.2) in a BioRad Mini-PROTEAN 3 cell electrophoresis system. Proteins were blotted onto nitrocellulose membrane by semi dry Western blot (Stack from top to bottom: 3x Wattman paper; SDS Gel; Nitrocellulose membrane; 3x Wattman paper) for $2 \mathrm{~h}$ at $100 \mathrm{~mA}$ in Blotbuffer (see 11.2). After applying blocking buffer for $1 \mathrm{~h}$ at room temperature and continuous shaking, the membrane was probed against SYD-2 and the loading control actin at 4 ${ }^{\circ} \mathrm{C}$ over night, continuously shaking. The polyclonal goat antibody against the $\mathrm{N}$ terminal region aa 30-88 of SYD-2 (Santa Cruz Biotechnology) and the polyclonal rabbit antibody (Sigma) were used in 1:500 dilution in blocking buffer. Peroxidasecoupled secondary antibodies (donkey anti-goat, Santa Cruz Biotechnology; goat anti-rabbit, The Jackson Laboratory) were used in 1:10000 dilution in blocking buffer and applied at room temperature for 45 minutes. Membranes were incubated in developing solution (see 11.2) for 2 minutes in the dark and imaged with Fujifilm LAS3000.

\subsection{Aldicarb assay}

Small agar plates $(\varnothing 3 \mathrm{~cm}$ ) were seeded with $50 \mu \mathrm{l}$ OP50 in the plate center and incubated overnight at $37^{\circ} \mathrm{C}$. Aldicarb (Sigma-Aldrich; 33386-100MG) was dissolved in $70 \%$ Ethanol. Concentration was adjusted to the weight of the agar and spread over the seeded plates. Ethanol evaporated for about 30 minutes from open plates. Plates were immediately used for assays. 30 adult worms were picked onto the bacteria lawn and paralysis was scored every 30 minutes. Animals were considered 
completely immobilized when also nose and tail showed no movement after body touch with a worm pick. Immobilized worms were picked off the plate and noted for each time point.

\subsection{Levamisole assay}

Levamisole of the desired concentration was added to the liquid agar and plates were poured and kept at $4^{\circ} \mathrm{C}$ until used. One day before use, about $50 \mu$ l of OP50 was dropped onto the middle of the plates and incubated at $37{ }^{\circ} \mathrm{C}$ over night to grow a thin bacteria lawn. 10 young adult animals were placed on the plates and motility was checked every 10 minutes by tapping the plates and body touches with a worm pick. Animals were considered completely immobilized when no nose or tail movement was detectable anymore.

\subsection{PCR mutagenesis and cloning}

Classic recombinant molecular biology techniques were used for molecular cloning (Maniatis et al., 1982). Bacteria and yeast strains, vectors and constructs are listed below in Table 2, 3 and 4. Constructs are described with primers used for amplification and restriction sites used for cloning. Sequences and description of all primers (Invitrogen) are listed in the Appendix 11.3.

Table 2 Bacteria and yeast strains used in study

\begin{tabular}{lll} 
Strain & Description & Source \\
\hline DH5a & $\begin{array}{l}\text { E.coli strain, competent cells used for } \\
\text { molecular cloning }\end{array}$ & Invitrogen \\
AH109 & $\begin{array}{l}\text { S. cerevisae strain used in yeast-2-hybrid } \\
\text { assays }\end{array}$ & Clontech \\
& $\begin{array}{l}\text { E. coli strain used as food for C. elegans } \\
\text { OP50 }\end{array}$ & (Brenner, 1974)
\end{tabular}

Table 3 Backbone vectors used in this study

\begin{tabular}{llll} 
Vector & Resistance & Description & Source \\
\hline pGEMT & Ampicillin & entry vector for TA cloning & Promega
\end{tabular}




\begin{tabular}{llll}
\hline L4440 & Ampicillin & vector used for subcloning & $\begin{array}{l}\text { Kindly provided } \\
\text { by Julie Ahringer }\end{array}$ \\
pGBKT7 & Kanamycin & $\begin{array}{l}\text { yeast-2-hybrid vector with DNA } \\
\text { binding domain }\end{array}$ & $\begin{array}{l}\text { Clontech } \\
\text { pGADT7 }\end{array}$ \\
& Ampicillin & $\begin{array}{l}\text { yeast-2-hybrid vector with DNA } \\
\text { activating domain }\end{array}$ & Clontech
\end{tabular}

$20 \mu \mathrm{l}$ of $50 \mathrm{mg} / \mathrm{mL} \quad \mathrm{X}$-Gal (5-Brom-4-chlor-3-indoxyl- $\beta$-D-galactopyranosid) in dimethylformamid and $10 \mu \mathrm{l}$ of $1 \mathrm{M}$ IPTG in $\mathrm{H}_{2} \mathrm{O}$ were dispersed onto pre-warmed ampicillin LB plates in case of pGMT vectors. For all other vectors, pre-warmed ampicillin LB plates were used.

In general, $100 \mu$ l of competent cells (DH5a) were thawed for each transformation. Complete ligation suspension or $1 \mu \mathrm{l}$ of construct for retransformation was added and incubated for 30 minutes on ice. Cells were heat shocked at $42{ }^{\circ} \mathrm{C}$ for 45 seconds and immediately put back on ice for 2 minutes. $900 \mu \mathrm{l}$ of (pre-warmed) LB medium (without ampicillin) was added and incubated on a shaking platform for $1 \mathrm{~h}$ at $37^{\circ} \mathrm{C}$; shaking speed approximately 800 rpm. After incubation, cells were centrifuged for 1 min at $12000 \mathrm{rpm}$, supernatant poured off and pellet resuspended in remaining medium. Cells were plated onto ampicillin plates and incubated at $37^{\circ} \mathrm{C}$ over night. In case of pGMT vectors, dark blue colonies contained empty vectors, white and light blue colonies probably vector and inserted fragment.

5 - 10 (white) colonies were picked and used for miniprep (Invitrogen, PureLink Quick Plasmid Mini Prep Kit). Purified plasmids were used for cloning. Constructs are listed in Table 4.

PCR amplification and PCR mutagenesis of syd-2 LH1 domain and elks-1 was done using Pfu-Ultra II Polymerase (Stratagene).

The pGEX-6 vector containing the DNA sequence of the LH1 domain was provided by Katrin Schwarze. LH1 wild type was amplified with primers oGQ 2183 and oGQ 2237 introducing restriction sites. After gel extraction, the fragment was digested and ligated into L4440 between Agel / Xhol sites at $6{ }^{\circ} \mathrm{C}$ over night (MKM 020). The LH1 fragment was then subcloned (Agel / Xhol) into pGADT7 and pGBKT7 vectors for yeast-2-hybrid screen (MKM 024 and MKM 025). 
The base exchange found in the LH1 domain of syd-2(ju487) mutants was generated by PCR mutagenesis. For mutagenesis and introduction of restriction sites primers oGQ 2181 and oGQ 2237 were used. Amplification of the mutagenic fragment was done with primers OGQ 2183 and oGQ 2237. After gel extraction, the fragment was digested and ligated into L4440 between Agel / Xhol sites at $6{ }^{\circ} \mathrm{C}$ over night (MKM 021). The mutant LH1 fragment was then subcloned (Agel / Xhol) into pGADT7 and pGBKT7 vectors for yeast-2-hybrid screen (MKM 026 and MKM 027).

The gene elks-1 was amplified in two parts from a $C$. elegans cDNA library (Proquest).

Amplification of the first part and introduction of restriction sites was done with primers OGQ 2038 and OGQ 2056. After adding a poly-A tail with Taq polymerase (Invitrogen), the fragment was ligated into a pGEM-T vector (Promega) at $6{ }^{\circ} \mathrm{C}$ over night (MMK 001). The procedure was the same for the second part of elks-1, but primers oGQ 2063 and oGQ 2068 were used (MMK 004). Ligation of elks-1 part 1 (Agel and BamHI), part 2 (BamHI and Xhol) and a L4440 vector (Agel / Xhol) was done at $6{ }^{\circ} \mathrm{C}$ over night (MMK 006). The complete elks-1 fragment was then subcloned between Agel / Xhol sites into pGADT7 and pGBKT7 for Yeast-two-hybrid screen (MMK 028 and MMK 029).

Table 4 Constructs generated in this study

Construct Plasmid and gene of interest Restriction sites

\begin{tabular}{lll}
\hline MKM 001 & pGEM-T elks-1 part 1 & TA cloning \\
\hline MKM 004 & pGEM-T elks-1 part 2 & TA cloning \\
\hline MKM 006 & L4440 elks-1 & Agel / Xhol \\
\hline MKM 020 & L4440 LH1 & Agel / Xhol \\
\hline MKM 021 & L4440 LH1 mutant & Agel / Xhol \\
\hline MKM 024 & pGADT7 LH1 & Agel / Xhol \\
\hline MKM 025 & pGBKT7 LH1 & Agel / Xhol \\
\hline MKM 026 & pGADT7 LH1 mutant & Agel / Xhol \\
\hline MKM 027 & pGBKT7 LH1 mutant & Agel / Xhol
\end{tabular}




\begin{tabular}{lll}
\hline MKM 028 & pGADT7 elks-1 & Agel / Xhol \\
\hline MKM 029 & pGBKT7 elks-1 & Agel / Xhol \\
\hline
\end{tabular}

\subsection{Egg-laying assay}

For each strain 10 adult animals were placed onto a fresh plate and let acclimate for $1 \mathrm{~h}$. Eggs were picked off the plates and discarded. Eggs were counted 2 hours later and averaged over 4 trials.

\subsection{Biochemistry (performed by collaborator Hidenori Taru)}

Following bacterial expression constructs for GST-fusion and His6-tagged proteins were generated using Gateway cloning system (Invitrogen) as described in the manufacturer's protocols: ELKS-1/pGEX-6P (pCZGY688), ELKS-1/pRSET B (pCZGY659), SYD-2 N (1-517) WT/pRSET (pCZGY682), and SYD-2 N (1-517) R184C/ pRSET (pCZGY683). SYD-2 fragment (aa 1-515) was cloned between BamHI and Sall sites of modified pGEX-6P vector to generate SYD-2 N WT/pGEX6P (pCZ757) or SYD-2 N R184C/pGEX-6P (pCZ758). SYD-2 91-207 was cloned between EcoRI and BamHI sites of pMAL-c2 vector to generate SYD-2 91207/pMAL-c2 or SYD-2 91-207 R184C/pMAL-c2. Plasmids constructions were confirmed by sequencing.

Recombinant GST-, His6-, MBP- fusion proteins were produced in E.coli BL21(DE3) with pGEX-6P, pRSET, pMAL-c2 plasmids incubating for $16 \mathrm{~h}$ at $22{ }^{\circ} \mathrm{C}$ in the presence of IPTG. Proteins were extracted by sonication in PBS $\mathrm{pH} 7.4$ with $0.1 \%$ TritonX-100, $1 \mathrm{mM}$ DTT, and protease inhibitors (Roche). For GST pull-down analysis, GST-fusion proteins in supernatant were immobilized to Glutathionesepharose (GE Healthcare), incubated with His6 proteins in supernatants for $3 \mathrm{~h}$ at 4 ${ }^{\circ} \mathrm{C}$, and washed for 4 times. Protein complexes were eluted and analyzed by ponceau S staining and western blotting using SuperSignal West Femto Substrate (Pierce) and mouse monoclonal anti-His6 (Novagen) antibody. For gel-filtration analysis, MBP-LH1 and MBP-LH1(R184C) were purified with Amylose resin (NEB) and $40 \mu \mathrm{g}$ of proteins were applied to an Agilent $1100 \mathrm{HPLC}$ with PROTEIN KW-803 column (Shodex) equilibrated with $20 \mathrm{mM}$ potassium phosphate pH6.8, $50 \mathrm{mM}$ 
sodium chloride at $0.5 \mathrm{~mL} / \mathrm{min}$ flow rate, detected by DAWN HELEOS 8+ and Optilab rEX (Wyatt).

\subsection{Yeast-two-hybrid screen}

$20 \mathrm{ml}$ of YAPD and 1 colony of freshly spread yeast (AH109) were grown over night at $30{ }^{\circ} \mathrm{C}$. For each transformation $700 \mu \mathrm{l}$ of over night yeast culture was used, centrifuged 2 minutes at $2000 \mathrm{rpm}$ and supernatant was removed. Cells were resuspended (vortexed) in $100 \mu$ l competence inducing One-Step-Buffer (see 11.2). About $2 \mu \mathrm{g}$ of potential interaction partner plasmids were added (one construct with DNA Binding domain, one with DNA activation domain). Mixture was incubate at room temperature for 15 minutes, then heat shocked at $45^{\circ} \mathrm{C}$ for 30 seconds and the suspension plated with glass beads onto selection plates. Plates were incubated at $30{ }^{\circ} \mathrm{C}$ for $2-3$ days. Growing colonies had both vectors included and were used for yeast two hybrid screen.

Spotting: Some colonies were scraped of the plates with an inoculating loop and dissolve into $1 \mathrm{ml} \mathrm{H}_{2} \mathrm{O}$ in a $1.5 \mathrm{ml}$ E-cup. $\mathrm{OD}_{600}$ was measured immediately after vortexing against a $\mathrm{H}_{2} \mathrm{O}$ blank using an Eppendorf BioPhotometer. Suspension were diluted with $\mathrm{H}_{2} \mathrm{O}$ to an $\mathrm{OD}_{600}$ of 0.2 in $100 \mu \mathrm{l}$. $5 \mu \mathrm{l}$ were spotted onto minimal plates (leu - trp, see 11.2) as control and selection plates (-leu - trp -his) to test for interaction.

\subsection{Electrophysiology (performed by collaborator Janet Richmond)}

Electrophysiological methods were performed as previously described (Richmond, 2009). Briefly, animals were immobilized with Histoacryl Blue glue. The ventral medial body wall muscles were exposed by a lateral cuticle incision with a glass needle. Body wall muscle recordings were done in the whole-cell voltage-clamp configuration (holding potential, $-60 \mathrm{mV}$ ) using an EPC-10 patch-clamp amplifier digitized at $1 \mathrm{kHz}$. The $5 \mathrm{mM} \mathrm{Ca}^{2+}$ extracellular solution consisted of $150 \mathrm{mM} \mathrm{NaCl}, 5$ $\mathrm{mM} \mathrm{KCl}, 5 \mathrm{mM} \mathrm{CaCl}_{2}, 4 \mathrm{mM} \mathrm{MgCl}_{2}, 10 \mathrm{mM}$ glucose, $5 \mathrm{mM}$ sucrose, and $15 \mathrm{mM}$ HEPES ( $\mathrm{pH} 7.3,-340$ mOsm). $\mathrm{Ca}^{2+}$ was replaced with $\mathrm{NaCl}$ in the $0.5 \mathrm{mM} \mathrm{Ca}{ }^{2+}$ extracellular solution. The patch pipette was filled with $120 \mathrm{mM} \mathrm{KCl,} 20 \mathrm{mM} \mathrm{KOH}, 4$ 
$\mathrm{mM} \mathrm{MgCl}_{2}, 5 \mathrm{mM}$ (N-tris[Hydroxymethyl] methyl-2-aminoethane-sulfonic acid), 0.25 $\mathrm{mM} \mathrm{CaCl}$, 4 mM Na${ }_{2}$ ATP, 36 mM sucrose, and 5 mM EGTA (pH 7.2, >315 mOsm). Data acquisition was done with Pulse software (HEKA, Southboro, Massachusetts, United States). Subsequent analysis and graphing was performed using Pulsefit (HEKA), Mini analysis (Synaptosoft Inc., Decatur, Georgia, United States) and Igor Pro (Wavemetrics, Lake Oswego, Oregon, United States).

\subsection{Statistics}

Data is displayed as mean +/- S.E.M. Statistical analysis was performed with Graph Pad prism (GraphPad Prism version 4 for Windows, GraphPad Software, La Jolla California USA, www.graphpad.com). Significance is given as p-value or asterisks after two-tailed Student's t-test or one-way ANOVA with Dunnett's post test ${ }^{*}=$ $p<0.05 ;{ }^{* *}=p<0.01 ;{ }^{* \star *}=p<0.001$ ) for samples where Gaussian distribution was given or assumed. Kruska-wallis test with Dunn's post test between selected pairs was applied for datasets that did not pass the normality test or for which normal distribution could not be assumed. 


\section{References}

Ahmari SE, Buchanan J, Smith SJ (Assembly of presynaptic active zones from cytoplasmic transport packets. Nat Neurosci 3:445-451.2000).

Ahnert-Hilger G, Holtje M, Pahner I, Winter S, Brunk I (Regulation of vesicular neurotransmitter transporters. Rev Physiol Biochem Pharmacol 150:140160.2003).

Altun ZF, Hall DH (2011) Nervous system, general description. In: WormAtlas.

Ashery U, Varoqueaux F, Voets T, Betz A, Thakur P, Koch H, Neher E, Brose N, Rettig J (Munc13-1 acts as a priming factor for large dense-core vesicles in bovine chromaffin cells. EMBO J 19:3586-3596.2000).

Betz $A$, Okamoto $M$, Benseler $F$, Brose $N$ (Direct interaction of the rat unc-13 homologue Munc13-1 with the N terminus of syntaxin. J Biol Chem 272:25202526.1997).

Betz A, Thakur P, Junge HJ, Ashery U, Rhee JS, Scheuss V, Rosenmund C, Rettig $\mathrm{J}$, Brose $\mathrm{N}$ (Functional interaction of the active zone proteins Munc13-1 and RIM1 in synaptic vesicle priming. Neuron 30:183-196.2001).

Brenner S (The genetics of Caenorhabditis elegans. Genetics 77:71-94.1974).

Brose N, Hofmann K, Hata Y, Sudhof TC (Mammalian homologues of Caenorhabditis elegans unc-13 gene define novel family of C2-domain proteins. J Biol Chem 270:25273-25280.1995).

Brose N, Petrenko AG, Sudhof TC, Jahn R (Synaptotagmin: a calcium sensor on the synaptic vesicle surface. Science 256:1021-1025.1992).

Bruses $\mathrm{JL}$ (Cadherin-mediated adhesion at the interneuronal synapse. Curr Opin Cell Biol 12:593-597.2000).

Byrd DT, Kawasaki M, Walcoff M, Hisamoto N, Matsumoto K, Jin Y (UNC-16, a JNKsignaling scaffold protein, regulates vesicle transport in C. elegans. Neuron 32:787-800.2001).

Cai T, Hirai H, Fukushige T, Yu P, Zhang G, Notkins AL, Krause M (Loss of the transcriptional repressor PAG-3/Gfi-1 results in enhanced neurosecretion that is dependent on the dense-core vesicle membrane protein IDA-1/IA-2. PLoS Genet 5:e1000447.2009).

Cases-Langhoff C, Voss B, Garner AM, Appeltauer U, Takei K, Kindler S, Veh RW, De Camilli P, Gundelfinger ED, Garner CC (Piccolo, a novel 420 kDa protein associated with the presynaptic cytomatrix. Eur J Cell Biol 69:214-223.1996).

Chalfie M, Sulston JE, White JG, Southgate E, Thomson JN, Brenner S (The neural circuit for touch sensitivity in Caenorhabditis elegans. J Neurosci 5:956964.1985).

Chalfie M, Tu Y, Euskirchen G, Ward WW, Prasher DC (Green fluorescent protein as a marker for gene expression. Science 263:802-805.1994).

Chavis P, Westbrook G (Integrins mediate functional pre- and postsynaptic maturation at a hippocampal synapse. Nature 411:317-321.2001).

Chen YA, Scheller RH (SNARE-mediated membrane fusion. Nat Rev Mol Cell Biol 2:98-106.2001).

Chisholm AD, Jin $Y$ (Neuronal differentiation in C. elegans. Curr Opin Cell Biol 17:682-689.2005).

Cho KI, Cai Y, Yi H, Yeh A, Aslanukov A, Ferreira PA (Association of the kinesinbinding domain of RanBP2 to KIF5B and KIF5C determines mitochondria localization and function. Traffic 8:1722-1735.2007). 
Chua JJ, Butkevich E, Worseck JM, Kittelmann M, Gronborg M, Behrmann E, Stelzl U, Pavlos NJ, Lalowski MM, Eimer S, Wanker EE, Klopfenstein DR, Jahn R (Phosphorylation-regulated axonal dependent transport of syntaxin 1 is mediated by a Kinesin-1 adapter. Proc Natl Acad Sci U S A 109:58625867.2012).

Coppola T, Magnin-Luthi S, Perret-Menoud V, Gattesco S, Schiavo G, Regazzi R (Direct interaction of the Rab3 effector RIM with Ca2+ channels, SNAP-25, and synaptotagmin. J Biol Chem 276:32756-32762.2001).

Dai Y, Taru H, Deken SL, Grill B, Ackley B, Nonet ML, Jin Y (SYD-2 Liprin-alpha organizes presynaptic active zone formation through ELKS. Nat Neurosci 9:1479-1487.2006).

de Bono M, Maricq AV (Neuronal substrates of complex behaviors in C. elegans. Annu Rev Neurosci 28:451-501.2005).

Deken SL, Vincent R, Hadwiger G, Liu Q, Wang ZW, Nonet ML (Redundant localization mechanisms of RIM and ELKS in Caenorhabditis elegans. J Neurosci 25:5975-5983.2005).

Desai CJ, Garrity PA, Keshishian H, Zipursky SL, Zinn K (The Drosophila SH2-SH3 adapter protein Dock is expressed in embryonic axons and facilitates synapse formation by the RP3 motoneuron. Development 126:1527-1535.1999).

Dick O, tom Dieck S, Altrock WD, Ammermuller J, Weiler R, Garner CC, Gundelfinger ED, Brandstatter $\mathrm{JH}$ (The presynaptic active zone protein bassoon is essential for photoreceptor ribbon synapse formation in the retina. Neuron 37:775-786.2003).

Ding M, Chao D, Wang G, Shen K (Spatial regulation of an E3 ubiquitin ligase directs selective synapse elimination. Science 317:947-951.2007).

Dixon SJ, Roy PJ (Muscle arm development in Caenorhabditis elegans. Development 132:3079-3092.2005).

Donnert G, Keller J, Medda R, Andrei MA, Rizzoli SO, Luhrmann R, Jahn R, Eggeling $C$, Hell SW (Macromolecular-scale resolution in biological fluorescence microscopy. Proc Natl Acad Sci U S A 103:11440-11445.2006).

Dresbach T, Qualmann B, Kessels MM, Garner CC, Gundelfinger ED (The presynaptic cytomatrix of brain synapses. Cell Mol Life Sci 58:94-116.2001).

Dubochet J, Adrian M, Chang JJ, Homo JC, Lepault J, McDowall AW, Schultz P (Cryo-electron microscopy of vitrified specimens. Q Rev Biophys 21:129228.1988).

Dulubova I, Lou X, Lu J, Huryeva I, Alam A, Schneggenburger R, Sudhof TC, Rizo J (A Munc13/RIM/Rab3 tripartite complex: from priming to plasticity? EMBO J 24:2839-2850.2005).

Dybbs M, Ngai J, Kaplan JM (Using microarrays to facilitate positional cloning: identification of tomosyn as an inhibitor of neurosecretion. PLoS Genet 1:616.2005).

Erni R, Rossell MD, Kisielowski C, Dahmen U (Atomic-resolution imaging with a sub50-pm electron probe. Phys Rev Lett 102:096101.2009).

Fares H, Greenwald I (Genetic analysis of endocytosis in Caenorhabditis elegans: coelomocyte uptake defective mutants. Genetics 159:133-145.2001).

Farsad K, Ringstad N, Takei K, Floyd SR, Rose K, De Camilli P (Generation of high curvature membranes mediated by direct endophilin bilayer interactions. J Cell Biol 155:193-200.2001).

Fasshauer D, Sutton RB, Brunger AT, Jahn R (Conserved structural features of the synaptic fusion complex: SNARE proteins reclassified as Q- and R-SNAREs. Proc Natl Acad Sci U S A 95:15781-15786.1998). 
Feeney CJ, Karunanithi S, Pearce J, Govind CK, Atwood HL (Motor nerve terminals on abdominal muscles in larval flesh flies, Sarcophaga bullata: Comparisons with Drosophila. Journal of Comparative Neurology 402:197-209.1998).

Fenster SD, Chung WJ, Zhai R, Cases-Langhoff C, Voss B, Garner AM, Kaempf U, Kindler S, Gundelfinger ED, Garner CC (Piccolo, a presynaptic zinc finger protein structurally related to bassoon. Neuron 25:203-214.2000).

Fernandez I, Arac D, Ubach J, Gerber SH, Shin O, Gao Y, Anderson RG, Sudhof TC, Rizo J (Three-dimensional structure of the synaptotagmin 1 C2B-domain: synaptotagmin 1 as a phospholipid binding machine. Neuron 32:10571069.2001).

Fernandez-Chacon R, Konigstorfer A, Gerber SH, Garcia J, Matos MF, Stevens CF, Brose N, Rizo J, Rosenmund C, Sudhof TC (Synaptotagmin I functions as a calcium regulator of release probability. Nature 410:41-49.2001).

Fiala JC (Reconstruct: a free editor for serial section microscopy. J Microsc 218:5261.2005).

Foster M, Sherrington CS (1897) A textbook of physiology, part three: The centralnervoussystem (7th ed.). London: MacMillan \& Co Ltd.

Fouquet W, Owald D, Wichmann C, Mertel S, Depner H, Dyba M, Hallermann S, Kittel RJ, Eimer S, Sigrist SJ (Maturation of active zone assembly by Drosophila Bruchpilot. J Cell Biol 186:129-145.2009).

Frank J, Wagenknecht T, McEwen BF, Marko M, Hsieh CE, Mannella CA (Threedimensional imaging of biological complexity. J Struct Biol 138:85-91.2002).

Frank M, Eiberger B, Janssen-Bienhold $U$, de Sevilla Muller LP, Tjarks A, Kim JS, Maschke S, Dobrowolski R, Sasse P, Weiler R, Fleischmann BK, Willecke K (Neuronal connexin-36 can functionally replace connexin-45 in mouse retina but not in the developing heart. J Cell Sci 123:3605-3615.2010).

Fujimoto K, Shibasaki T, Yokoi N, Kashima Y, Matsumoto M, Sasaki T, Tajima N, Iwanaga $\mathrm{T}$, Seino $\mathrm{S}$ (Piccolo, a $\mathrm{Ca} 2+$ sensor in pancreatic beta-cells. Involvement of cAMP-GEFII.Rim2. Piccolo complex in cAMP-dependent exocytosis. J Biol Chem 277:50497-50502.2002).

Gage PW (Signal transmission in ligand-gated receptors. Immunol Cell Biol 76:436440.1998).

Gally C, Bessereau JL ([C. elegans: of neurons and genes]. Med Sci (Paris) 19:725734.2003).

Gally C, Eimer S, Richmond JE, Bessereau JL (A transmembrane protein required for acetylcholine receptor clustering in Caenorhabditis elegans. Nature 431:578-582.2004).

Garner CC, Zhai RG, Gundelfinger ED, Ziv NE (Molecular mechanisms of CNS synaptogenesis. Trends Neurosci 25:243-251.2002).

Goldstein LS, Yang Z (Microtubule-based transport systems in neurons: the roles of kinesins and dyneins. Annu Rev Neurosci 23:39-71.2000).

Goodman MB (Mechanosensation. WormBook 1-14.2006).

Gracheva EO, Burdina AO, Holgado AM, Berthelot-Grosjean M, Ackley BD, Hadwiger G, Nonet ML, Weimer RM, Richmond JE (Tomosyn inhibits synaptic vesicle priming in Caenorhabditis elegans. PLoS Biol 4:e261.2006).

Gracheva EO, Hadwiger G, Nonet ML, Richmond JE (Direct interactions between C. elegans RAB-3 and Rim provide a mechanism to target vesicles to the presynaptic density. Neurosci Lett 444:137-142.2008).

Greener T, Grant B, Zhang Y, Wu X, Greene LE, Hirsh D, Eisenberg E (Caenorhabditis elegans auxilin: a J-domain protein essential for clathrinmediated endocytosis in vivo. Nat Cell Biol 3:215-219.2001). 
Guillaud L, Wong R, Hirokawa N (Disruption of KIF17-Mint1 interaction by CaMKIIdependent phosphorylation: a molecular model of kinesin-cargo release. Nat Cell Biol 10:19-29.2008).

Hall DH, Hedgecock EM (Kinesin-related gene unc-104 is required for axonal transport of synaptic vesicles in C. elegans. Cell 65:837-847.1991).

Hallam SJ, Goncharov A, McEwen J, Baran R, Jin Y (SYD-1, a presynaptic protein with PDZ, C2 and rhoGAP-like domains, specifies axon identity in C. elegans. Nat Neurosci 5:1137-1146.2002).

Hannah MJ, Schmidt AA, Huttner WB (Synaptic vesicle biogenesis. Annu Rev Cell Dev Biol 15:733-798.1999).

Harlow ML, Ress D, Stoschek A, Marshall RM, McMahan UJ (The architecture of active zone material at the frog's neuromuscular junction. Nature 409:479484.2001).

Harris TW, Hartwieg E, Horvitz HR, Jorgensen EM (Mutations in synaptojanin disrupt synaptic vesicle recycling. J Cell Biol 150:589-600.2000).

Haucke V, De Camilli P (AP-2 recruitment to synaptotagmin stimulated by tyrosinebased endocytic motifs. Science 285:1268-1271.1999).

Haucke V, Klingauf J (2007) Endocytosis in neurons. In: Protein Trafficking in Neurons(Bean, A., ed) Amsterdam, Boston: Elsevier - Academic Press.

Hazuka CD, Foletti DL, Hsu SC, Kee Y, Hopf FW, Scheller RH (The sec6/8 complex is located at neurite outgrowth and axonal synapse-assembly domains. J Neurosci 19:1324-1334.1999).

Hedgecock EM, Culotti JG, Hall DH (The unc-5, unc-6, and unc-40 genes guide circumferential migrations of pioneer axons and mesodermal cells on the epidermis in C. elegans. Neuron 4:61-85.1990).

Hedgecock EM, Russell RL (Normal and mutant thermotaxis in the nematode Caenorhabditis elegans. Proc Natl Acad Sci U S A 72:4061-4065.1975).

Hirokawa N, Noda Y, Tanaka Y, Niwa S (Kinesin superfamily motor proteins and intracellular transport. Nat Rev Mol Cell Biol 10:682-696.2009).

Hong W (SNAREs and traffic. Biochim Biophys Acta 1744:493-517.2005).

Hoogenraad CC, Feliu-Mojer MI, Spangler SA, Milstein AD, Dunah AW, Hung AY, Sheng $M$ (Liprinalpha1 degradation by calcium/calmodulin-dependent protein kinase II regulates LAR receptor tyrosine phosphatase distribution and dendrite development. Dev Cell 12:587-602.2007).

Huttner WB, Schmidt A (Lipids, lipid modification and lipid-protein interaction in membrane budding and fission--insights from the roles of endophilin A1 and synaptophysin in synaptic vesicle endocytosis. Curr Opin Neurobiol 10:543551.2000).

Jahn R, Lang T, Sudhof TC (Membrane fusion. Cell 112:519-533.2003).

Jahn R, Scheller RH (SNAREs--engines for membrane fusion. Nat Rev Mol Cell Biol 7:631-643.2006).

Jorgensen EM, Hartwieg E, Schuske K, Nonet ML, Jin Y, Horvitz HR (Defective recycling of synaptic vesicles in synaptotagmin mutants of Caenorhabditis elegans. Nature 378:196-199.1995).

Jung N, Haucke V (Clathrin-mediated endocytosis at synapses. Traffic 8:11291136.2007).

Kaufmann N, DeProto J, Ranjan R, Wan H, Van Vactor D (Drosophila liprin-alpha and the receptor phosphatase Dlar control synapse morphogenesis. Neuron 34:27-38.2002). 
Khimich D, Nouvian R, Pujol R, Tom Dieck S, Egner A, Gundelfinger ED, Moser T (Hair cell synaptic ribbons are essential for synchronous auditory signalling. Nature 434:889-894.2005).

Kittel RJ, Wichmann C, Rasse TM, Fouquet W, Schmidt M, Schmid A, Wagh DA, Pawlu C, Kellner RR, Willig KI, Hell SW, Buchner E, Heckmann M, Sigrist SJ (Bruchpilot promotes active zone assembly, Ca2+ channel clustering, and vesicle release. Science 312:1051-1054.2006).

Klassen MP, Shen K (Wnt signaling positions neuromuscular connectivity by inhibiting synapse formation in C. elegans. Cell 130:704-716.2007).

Klassen MP, Wu YE, Maeder Cl, Nakae I, Cueva JG, Lehrman EK, Tada M, GengyoAndo K, Wang GJ, Goodman M, Mitani S, Kontani K, Katada T, Shen K (An Arf-like small $G$ protein, ARL-8, promotes the axonal transport of presynaptic cargoes by suppressing vesicle aggregation. Neuron 66:710-723.2010).

Kloepper TH, Kienle CN, Fasshauer D (An elaborate classification of SNARE proteins sheds light on the conservation of the eukaryotic endomembrane system. Mol Biol Cell 18:3463-3471.2007).

Klopfenstein DR, Tomishige $M$, Stuurman N, Vale RD (Role of phosphatidylinositol(4,5)bisphosphate organization in membrane transport by the Unc104 kinesin motor. Cell 109:347-358.2002).

Klopfenstein DR, Vale RD (The lipid binding pleckstrin homology domain in UNC-104 kinesin is necessary for synaptic vesicle transport in Caenorhabditis elegans. Mol Biol Cell 15:3729-3739.2004).

Ko J, Kim S, Valtschanoff JG, Shin H, Lee JR, Sheng M, Premont RT, Weinberg RJ, Kim E (Interaction between liprin-alpha and GIT1 is required for AMPA receptor targeting. J Neurosci 23:1667-1677.2003a).

Ko J, Na M, Kim S, Lee JR, Kim E (Interaction of the ERC family of RIM-binding proteins with the liprin-alpha family of multidomain proteins. J Biol Chem 278:42377-42385.2003b).

Koch H, Hofmann K, Brose N (Definition of Munc13-homology-domains and characterization of a novel ubiquitously expressed Munc13 isoform. Biochem $\mathrm{J}$ 349:247-253.2000).

Kohn RE, Duerr JS, McManus JR, Duke A, Rakow TL, Maruyama H, Moulder G, Maruyama IN, Barstead RJ, Rand JB (Expression of multiple UNC-13 proteins in the Caenorhabditis elegans nervous system. Mol Biol Cell 11:34413452.2000).

Koushika SP, Richmond JE, Hadwiger G, Weimer RM, Jorgensen EM, Nonet ML (A post-docking role for active zone protein Rim. Nat Neurosci 4:997-1005.2001).

Koushika SP, Schaefer AM, Vincent R, Willis JH, Bowerman B, Nonet ML (Mutations in Caenorhabditis elegans cytoplasmic dynein components reveal specificity of neuronal retrograde cargo. J Neurosci 24:3907-3916.2004).

Kremer JR, Mastronarde DN, Mclntosh JR (Computer visualization of threedimensional image data using IMOD. J Struct Biol 116:71-76.1996).

Kumar J, Choudhary BC, Metpally R, Zheng Q, Nonet ML, Ramanathan S, Klopfenstein DR, Koushika SP (The Caenorhabditis elegans Kinesin-3 motor UNC-104/KIF1A is degraded upon loss of specific binding to cargo. PLoS Genet 6:e1001200.2010).

Lawrence A, Bouwer JC, Perkins G, Ellisman MH (Transform-based backprojection for volume reconstruction of large format electron microscope tilt series. $\mathrm{J}$ Struct Biol 154:144-167.2006).

Lenzi D, von Gersdorff H (Structure suggests function: the case for synaptic ribbons as exocytotic nanomachines. Bioessays 23:831-840.2001). 
Lin W, Burgess RW, Dominguez B, Pfaff SL, Sanes JR, Lee KF (Distinct roles of nerve and muscle in postsynaptic differentiation of the neuromuscular synapse. Nature 410:1057-1064.2001).

Lupetti P, Evangelista V, Barsanti L, Passarelli V, Gualtieri P (2005) Cryotechniques for Electron Microscopy: A Minireview

From Cells to Proteins: Imaging Nature across Dimensions. vol. 3, pp 53-70: Springer Netherlands.

Mahoney TR, Liu Q, Itoh T, Luo S, Hadwiger G, Vincent R, Wang ZW, Fukuda M, Nonet ML (Regulation of synaptic transmission by RAB-3 and RAB-27 in Caenorhabditis elegans. Mol Biol Cell 17:2617-2625.2006).

Maniatis T, Fritsch EF, Sambrook J (1982) Molecular cloning : a laboratory manual. Cold Spring Harbor, N.Y.: Cold Spring Harbor Laboratory.

Martens S, Kozlov MM, McMahon HT (How synaptotagmin promotes membrane fusion. Science 316:1205-1208.2007).

Martin TF, Kowalchyk JA (Docked secretory vesicles undergo Ca2+-activated exocytosis in a cell-free system. J Biol Chem 272:14447-14453.1997).

McDonald KL, Auer M (High-pressure freezing, cellular tomography, and structural cell biology. Biotechniques 41:137, 139, 141 passim.2006).

McIntire SL, Jorgensen E, Kaplan J, Horvitz HR (The GABAergic nervous system of Caenorhabditis elegans. Nature 364:337-341.1993).

McIntosh R, Nicastro D, Mastronarde D (New views of cells in 3D: an introduction to electron tomography. Trends Cell Biol 15:43-51.2005).

Miller KE, DeProto J, Kaufmann N, Patel BN, Duckworth A, Van Vactor D (Direct observation demonstrates that Liprin-alpha is required for trafficking of synaptic vesicles. Curr Biol 15:684-689.2005).

Miller TM, Heuser JE (Endocytosis of synaptic vesicle membrane at the frog neuromuscular junction. J Cell Biol 98:685-698.1984).

Mittelstaedt T, Alvarez-Baron E, Schoch S (RIM proteins and their role in synapse function. Biol Chem 391:599-606.2010).

Moor H (1987) Theory and practice of high pressure freezing. In: Cryotechniques in biological electron microscopy(Steinbrecht, R. A. and Zierold, K., eds), pp 175191 Berlin; New York: Springer-Verlag.

Nakata K, Abrams B, Grill B, Goncharov A, Huang X, Chisholm AD, Jin Y (Regulation of a DLK-1 and p38 MAP kinase pathway by the ubiquitin ligase RPM-1 is required for presynaptic development. Cell 120:407-420.2005).

Nakata T, Kitamura Y, Shimizu K, Tanaka S, Fujimori M, Yokoyama S, Ito K, Emi M (Fusion of a novel gene, ELKS, to RET due to translocation $t(10 ; 12)(q 11 ; p 13)$ in a papillary thyroid carcinoma. Genes Chromosomes Cancer 25:97103.1999).

Nakata T, Yokota T, Emi M, Minami S (Differential expression of multiple isoforms of the ELKS mRNAs involved in a papillary thyroid carcinoma. Genes Chromosomes Cancer 35:30-37.2002).

Nangaku M, Sato-Yoshitake R, Okada Y, Noda Y, Takemura R, Yamazaki H, Hirokawa N (KIF1B, a novel microtubule plus end-directed monomeric motor protein for transport of mitochondria. Cell 79:1209-1220.1994).

Nonet ML (Visualization of synaptic specializations in live C. elegans with synaptic vesicle protein-GFP fusions. J Neurosci Methods 89:33-40.1999).

Ohtsuka T, Takao-Rikitsu E, Inoue E, Inoue M, Takeuchi M, Matsubara K, DeguchiTawarada M, Satoh K, Morimoto K, Nakanishi H, Takai Y (Cast: a novel protein of the cytomatrix at the active zone of synapses that forms a ternary complex with RIM1 and munc13-1. J Cell Biol 158:577-590.2002). 
Okada $\mathrm{Y}$, Yamazaki H, Sekine-Aizawa $\mathrm{Y}$, Hirokawa $\mathrm{N}$ (The neuron-specific kinesin superfamily protein KIF1A is a unique monomeric motor for anterograde axonal transport of synaptic vesicle precursors. Cell 81:769-780.1995).

Pack-Chung E, Kurshan PT, Dickman DK, Schwarz TL (A Drosophila kinesin required for synaptic bouton formation and synaptic vesicle transport. Nat Neurosci 10:980-989.2007).

Paradis E, Claude J, Strimmer K (APE: Analyses of Phylogenetics and Evolution in R language. Bioinformatics 20:289-290.2004).

Patel MR, Lehrman EK, Poon VY, Crump JG, Zhen M, Bargmann Cl, Shen K (Hierarchical assembly of presynaptic components in defined C. elegans synapses. Nat Neurosci 9:1488-1498.2006).

Patel MR, Shen K (RSY-1 is a local inhibitor of presynaptic assembly in C. elegans. Science 323:1500-1503.2009).

Perin MS, Fried VA, Mignery GA, Jahn R, Sudhof TC (Phospholipid binding by a synaptic vesicle protein homologous to the regulatory region of protein kinase C. Nature 345:260-263.1990).

Peters A, Palay SL (The morphology of synapses. J Neurocytol 25:687-700.1996).

Pfeffer SR (Transport-vesicle targeting: tethers before SNAREs. Nat Cell Biol 1:E1722.1999).

Phillips GR, Huang JK, Wang Y, Tanaka H, Shapiro L, Zhang W, Shan WS, Arndt K, Frank M, Gordon RE, Gawinowicz MA, Zhao Y, Colman DR (The presynaptic particle web: ultrastructure, composition, dissolution, and reconstitution. Neuron 32:63-77.2001).

Pierce-Shimomura JT, Morse TM, Lockery SR (The fundamental role of pirouettes in Caenorhabditis elegans chemotaxis. J Neurosci 19:9557-9569.1999).

Plunkett JA, Simmons RB, Walthall WW (Dynamic interactions between nerve and muscle in Caenorhabditis elegans. Dev Biol 175:154-165.1996).

Pobbati AV, Stein A, Fasshauer D (N- to C-terminal SNARE complex assembly promotes rapid membrane fusion. Science 313:673-676.2006).

Poon VY, Klassen MP, Shen K (UNC-6/netrin and its receptor UNC-5 locally exclude presynaptic components from dendrites. Nature 455:669-673.2008).

Prokop A, Meinertzhagen IA (Development and structure of synaptic contacts in Drosophila. Semin Cell Dev Biol 17:20-30.2006).

Regus-Leidig H, Tom Dieck S, Specht D, Meyer L, Brandstatter JH (Early steps in the assembly of photoreceptor ribbon synapses in the mouse retina: the involvement of precursor spheres. J Comp Neurol 512:814-824.2009).

Rettig J, Neher E (Emerging roles of presynaptic proteins in $\mathrm{Ca}++$-triggered exocytosis. Science 298:781-785.2002).

Reynolds ES (The use of lead citrate at high $\mathrm{pH}$ as an electron-opaque stain in electron microscopy. J Cell Biol 17:208-212.1963).

Richmond J (Synaptic function. WormBook 1-14.2005).

Richmond $\mathrm{J}$ (Dissecting and recording from the $C$. Elegans neuromuscular junction. $\mathrm{J}$ Vis Exp.2009).

Richmond JE, Jorgensen EM (One GABA and two acetylcholine receptors function at the $C$. elegans neuromuscular junction. Nat Neurosci 2:791-797.1999).

Riddle DL, Blumenthal T, Meyer BJ, Priess JR (1997) C. elegans II. 2nd edition. Cold Spring Harbor, NY: Cold Spring Harbor Laboratory Press.

Rittweger E, Han KY, Irvine SE, Eggeling C, Hell SW (STED microscopy reveals crystal colour centres with nanometric resolution. Nat Photon 3:144-147.2009). 
Rizzoli SO, Bethani I, Zwilling D, Wenzel D, Siddiqui TJ, Brandhorst D, Jahn R (Evidence for early endosome-like fusion of recently endocytosed synaptic vesicles. Traffic 7:1163-1176.2006).

Rizzoli SO, Betz WJ (Synaptic vesicle pools. Nat Rev Neurosci 6:57-69.2005).

Rizzoli SO, Jahn R (Kiss-and-run, collapse and 'readily retrievable' vesicles. Traffic 8:1137-1144.2007).

Roos J, Kelly RB (Preassembly and transport of nerve terminals: a new concept of axonal transport. Nat Neurosci 3:415-417.2000).

Rostaing P, Real E, Siksou L, Lechaire JP, Boudier T, Boeckers TM, Gertler F, Gundelfinger ED, Triller A, Marty S (Analysis of synaptic ultrastructure without fixative using high-pressure freezing and tomography. Eur $\mathrm{J}$ Neurosci 24:3463-3474.2006).

Rostaing P, Weimer RM, Jorgensen EM, Triller A, Bessereau JL (Preservation of immunoreactivity and fine structure of adult $C$. elegans tissues using highpressure freezing. J Histochem Cytochem 52:1-12.2004).

Sakamoto R, Byrd DT, Brown HM, Hisamoto N, Matsumoto K, Jin $Y$ (The Caenorhabditis elegans UNC-14 RUN domain protein binds to the kinesin-1 and UNC-16 complex and regulates synaptic vesicle localization. Mol Biol Cell 16:483-496.2005).

Schikorski T, Stevens CF (Morphological correlates of functionally defined synaptic vesicle populations. Nat Neurosci 4:391-395.2001).

Schmid SL (Clathrin-coated vesicle formation and protein sorting: an integrated process. Annu Rev Biochem 66:511-548.1997).

Schmitz F, Konigstorfer A, Sudhof TC (RIBEYE, a component of synaptic ribbons: a protein's journey through evolution provides insight into synaptic ribbon function. Neuron 28:857-872.2000).

Schoch S, Castillo PE, Jo T, Mukherjee K, Geppert M, Wang Y, Schmitz F, Malenka RC, Sudhof TC (RIM1alpha forms a protein scaffold for regulating neurotransmitter release at the active zone. Nature 415:321-326.2002).

Schoch S, Gundelfinger ED (Molecular organization of the presynaptic active zone. Cell Tissue Res 326:379-391.2006).

Schuske K, Beg AA, Jorgensen EM (The GABA nervous system in C. elegans. Trends Neurosci 27:407-414.2004).

Schuske KR, Richmond JE, Matthies DS, Davis WS, Runz S, Rube DA, van der Bliek AM, Jorgensen EM (Endophilin is required for synaptic vesicle endocytosis by localizing synaptojanin. Neuron 40:749-762.2003).

Serra-Pages C, Medley QG, Tang M, Hart A, Streuli M (Liprins, a family of LAR transmembrane protein-tyrosine phosphatase-interacting proteins. J Biol Chem 273:15611-15620.1998).

Shakes DC, Allen AK, Albert KM, Golden A (emb-1 encodes the APC16 subunit of the Caenorhabditis elegans anaphase-promoting complex. Genetics 189:549560.2011).

Shapira M, Zhai RG, Dresbach T, Bresler T, Torres VI, Gundelfinger ED, Ziv NE, Garner CC (Unitary assembly of presynaptic active zones from PiccoloBassoon transport vesicles. Neuron 38:237-252.2003).

Shen K, Bargmann Cl (The immunoglobulin superfamily protein SYG-1 determines the location of specific synapses in C. elegans. Cell 112:619-630.2003).

Shen K, Fetter RD, Bargmann Cl (Synaptic specificity is generated by the synaptic guidepost protein SYG-2 and its receptor, SYG-1. Cell 116:869-881.2004). 
Shibasaki T, Sunaga Y, Fujimoto K, Kashima Y, Seino S (Interaction of ATP sensor, cAMP sensor, Ca2+ sensor, and voltage-dependent $\mathrm{Ca} 2+$ channel in insulin granule exocytosis. J Biol Chem 279:7956-7961.2004).

Shimizu Y, Morii H, Arisaka F, Tanokura M (Stalk region of kinesin-related protein Unc104 has moderate ability to form coiled-coil dimer. Biochem Biophys Res Commun 337:868-874.2005).

Shin H, Wyszynski M, Huh KH, Valtschanoff JG, Lee JR, Ko J, Streuli M, Weinberg RJ, Sheng M, Kim E (Association of the kinesin motor KIF1A with the multimodular protein liprin-alpha. J Biol Chem 278:11393-11401.2003).

Sieburth D, Ch'ng Q, Dybbs M, Tavazoie M, Kennedy S, Wang D, Dupuy D, Rual JF, Hill DE, Vidal M, Ruvkun G, Kaplan JM (Systematic analysis of genes required for synapse structure and function. Nature 436:510-517.2005).

Siksou L, Varoqueaux F, Pascual O, Triller A, Brose N, Marty S (A common molecular basis for membrane docking and functional priming of synaptic vesicles. Eur J Neurosci 30:49-56.2009).

Söllner T, Whiteheart SW, Brunner M, Erdjument-Bromage H, Geromanos S, Tempst $\mathrm{P}$, Rothman JE (SNAP receptors implicated in vesicle targeting and fusion. Nature 362:318-324.1993).

Sosa H, Popp D, Ouyang G, Huxley HE (Ultrastructure of skeletal muscle fibers studied by a plunge quick freezing method: myofilament lengths. Biophys $\mathrm{J}$ 67:283-292.1994).

Spangler SA, Hoogenraad CC (Liprin-alpha proteins: scaffold molecules for synapse maturation. Biochem Soc Trans 35:1278-1282.2007).

Stigloher $\mathrm{C}$, Zhan $\mathrm{H}$, Zhen M, Richmond J, Bessereau JL (The presynaptic dense projection of the Caenorhabditis elegans cholinergic neuromuscular junction localizes synaptic vesicles at the active zone through SYD-2/liprin and UNC10/RIM-dependent interactions. J Neurosci 31:4388-4396.2011).

Stretton AO (Anatomy and development of the somatic musculature of the nematode Ascaris. J Exp Biol 64:773-788.1976).

Stroud RM, McCarthy MP, Shuster M (Nicotinic acetylcholine receptor superfamily of ligand-gated ion channels. Biochemistry 29:11009-11023.1990).

Sudhof TC (The synaptic vesicle cycle. Annu Rev Neurosci 27:509-547.2004).

Sudhof TC, Rothman JE (Membrane fusion: grappling with SNARE and SM proteins. Science 323:474-477.2009).

Sulston JE, Horvitz HR (Post-embryonic cell lineages of the nematode, Caenorhabditis elegans. Dev Biol 56:110-156.1977).

Sutton RB, Fasshauer D, Jahn R, Brunger AT (Crystal structure of a SNARE complex involved in synaptic exocytosis at 2.4 A resolution. Nature 395:347-353.1998).

Sweitzer SM, Hinshaw JE (Dynamin undergoes a GTP-dependent conformational change causing vesiculation. Cell 93:1021-1029.1998).

Takamori S, Holt M, Stenius K, Lemke EA, Gronborg M, Riedel D, Urlaub H, Schenck S, Brugger B, Ringler P, Muller SA, Rammner B, Grater F, Hub JS, De Groot BL, Mieskes G, Moriyama Y, Klingauf J, Grubmuller H, Heuser J, Wieland F, Jahn R (Molecular anatomy of a trafficking organelle. Cell 127:831-846.2006).

Takao-Rikitsu E, Mochida S, Inoue E, Deguchi-Tawarada M, Inoue M, Ohtsuka T, Takai $Y$ (Physical and functional interaction of the active zone proteins, CAST, RIM1, and Bassoon, in neurotransmitter release. J Cell Biol 164:301311.2004).

Tao-Cheng JH, Du J, McBain CJ (Snap-25 is polarized to axons and abundant along the axolemma: an immunogold study of intact neurons. J Neurocytol 29:6777.2000). 
Taru $\mathrm{H}$, Jin $\mathrm{Y}$ (The Liprin homology domain is essential for the homomeric interaction of SYD-2/Liprin-alpha protein in presynaptic assembly. J Neurosci 31:1626116268.2011).

Toda H, Mochizuki H, Flores R, 3rd, Josowitz R, Krasieva TB, Lamorte VJ, Suzuki E, Gindhart JG, Furukubo-Tokunaga K, Tomoda T (UNC-51/ATG1 kinase regulates axonal transport by mediating motor-cargo assembly. Genes Dev 22:3292-3307.2008).

tom Dieck S, Sanmarti-Vila L, Langnaese K, Richter K, Kindler S, Soyke A, Wex H, Smalla KH, Kampf U, Franzer JT, Stumm M, Garner CC, Gundelfinger ED (Bassoon, a novel zinc-finger CAG/glutamine-repeat protein selectively localized at the active zone of presynaptic nerve terminals. J Cell Biol 142:499-509.1998).

Tomishige M, Klopfenstein DR, Vale RD (Conversion of Unc104/KIF1A kinesin into a processive motor after dimerization. Science 297:2263-2267.2002).

Tsien RY (The green fluorescent protein. Annu Rev Biochem 67:509-544.1998).

Ungewickell E, Ungewickell H, Holstein SE, Lindner R, Prasad K, Barouch W, Martin $B$, Greene LE, Eisenberg $E$ (Role of auxilin in uncoating clathrin-coated vesicles. Nature 378:632-635.1995).

Vale RD, Milligan RA (The way things move: looking under the hood of molecular motor proteins. Science 288:88-95.2000).

Vale RD, Reese TS, Sheetz MP (Identification of a novel force-generating protein, kinesin, involved in microtubule-based motility. Cell 42:39-50.1985).

van Roessel P, Elliott DA, Robinson IM, Prokop A, Brand AH (Independent regulation of synaptic size and activity by the anaphase-promoting complex. Cell 119:707-718.2004).

Verhage M, Maia AS, Plomp JJ, Brussaard AB, Heeroma $\mathrm{JH}$, Vermeer $\mathrm{H}$, Toonen RF, Hammer RE, van den Berg TK, Missler M, Geuze HJ, Sudhof TC (Synaptic assembly of the brain in the absence of neurotransmitter secretion. Science 287:864-869.2000).

Wagh DA, Rasse TM, Asan E, Hofbauer A, Schwenkert I, Durrbeck H, Buchner S, Dabauvalle MC, Schmidt M, Qin G, Wichmann C, Kittel R, Sigrist SJ, Buchner $\mathrm{E}$ (Bruchpilot, a protein with homology to ELKS/CAST, is required for structural integrity and function of synaptic active zones in Drosophila. Neuron 49:833844.2006).

Wagner OI, Esposito A, Kohler B, Chen CW, Shen CP, Wu GH, Butkevich E, Mandalapu S, Wenzel D, Wouters FS, Klopfenstein DR (Synaptic scaffolding protein SYD-2 clusters and activates kinesin-3 UNC-104 in C. elegans. Proc Natl Acad Sci U S A 106:19605-19610.2009).

Wang Y, Liu X, Biederer T, Sudhof TC (A family of RIM-binding proteins regulated by alternative splicing: Implications for the genesis of synaptic active zones. Proc Natl Acad Sci U S A 99:14464-14469.2002).

Wang Y, Okamoto M, Schmitz F, Hofmann K, Sudhof TC (Rim is a putative Rab3 effector in regulating synaptic-vesicle fusion. Nature 388:593-598.1997).

Weber T, Zemelman BV, McNew JA, Westermann B, Gmachl M, Parlati F, Sollner $\mathrm{TH}$, Rothman JE (SNAREpins: minimal machinery for membrane fusion. Cell 92:759-772.1998).

Weimer RM (Preservation of $C$. elegans tissue via high-pressure freezing and freezesubstitution for ultrastructural analysis and immunocytochemistry. Methods Mol Biol 351:203-221.2006). 
Weimer RM, Gracheva EO, Meyrignac O, Miller KG, Richmond JE, Bessereau JL (UNC-13 and UNC-10/rim localize synaptic vesicles to specific membrane domains. J Neurosci 26:8040-8047.2006).

White JG, Southgate E, Thomson JN, Brenner S (The structure of the nervous system of the nematode Caenorhabditis elegans. Philos Trans R Soc Lond B Biol Sci 314:1-340.1986).

Wichmann C, Sigrist SJ (The active zone T-bar--a plasticity module? J Neurogenet 24:133-145.2010).

Wojcik SM, Brose N (Regulation of membrane fusion in synaptic excitation-secretion coupling: speed and accuracy matter. Neuron 55:11-24.2007).

Wucherpfennig T, Wilsch-Brauninger M, Gonzalez-Gaitan M (Role of Drosophila Rab5 during endosomal trafficking at the synapse and evoked neurotransmitter release. J Cell Biol 161:609-624.2003).

Wyszynski M, Kim E, Dunah AW, Passafaro M, Valtschanoff JG, Serra-Pages C, Streuli M, Weinberg RJ, Sheng M (Interaction between GRIP and liprinalpha/SYD2 is required for AMPA receptor targeting. Neuron 34:39-52.2002).

Yeh E, Kawano T, Weimer RM, Bessereau JL, Zhen M (Identification of genes involved in synaptogenesis using a fluorescent active zone marker in Caenorhabditis elegans. J Neurosci 25:3833-3841.2005).

Yonekawa Y, Harada A, Okada Y, Funakoshi T, Kanai Y, Takei Y, Terada S, Noda T, Hirokawa N (Defect in synaptic vesicle precursor transport and neuronal cell death in KIF1A motor protein-deficient mice. J Cell Biol 141:431-441.1998).

Zahn TR, Angleson JK, MacMorris MA, Domke E, Hutton JF, Schwartz C, Hutton JC (Dense core vesicle dynamics in Caenorhabditis elegans neurons and the role of kinesin UNC-104. Traffic 5:544-559.2004).

Zhai RG, Bellen $\mathrm{HJ}$ (The architecture of the active zone in the presynaptic nerve terminal. Physiology (Bethesda) 19:262-270.2004).

Zhai RG, Vardinon-Friedman H, Cases-Langhoff C, Becker B, Gundelfinger ED, Ziv NE, Garner CC (Assembling the presynaptic active zone: a characterization of an active one precursor vesicle. Neuron 29:131-143.2001).

Zhen $M$, Huang $X$, Bamber $B$, Jin $Y$ (Regulation of presynaptic terminal organization by $C$. elegans RPM-1, a putative guanine nucleotide exchanger with a RING$\mathrm{H} 2$ finger domain. Neuron 26:331-343.2000).

Zhen $\mathrm{M}$, Jin $\mathrm{Y}$ (The liprin protein SYD-2 regulates the differentiation of presynaptic termini in C. elegans. Nature 401:371-375.1999). 


\section{Appendix}

\subsection{Table of abbreviations}

$\quad$ mikrometer

aa aminoacid

ACh acetylcholine

AMPA $\quad \alpha$-amino-3-hydroxy-5-methylisoxazole-4-propionic acid

APC/C anaphase-promoting complex/cyclosome

ATP adenosine triphosphate

AZ Active Zone

BWM body wall muscles

CaMKII calcium/calmodulin-dependent protein kinase II

CAST CAZ-associated structural protein

CAZ cytomatrix at the active zone

CC coiled coil

CCD charge-coupled device

DCV dense core vesicle

DP dense projection

ELKS glutamate $(E)$, leucine $(\mathrm{L})$, lysine $(\mathrm{K})$ and serine $(\mathrm{S})$ rich proteins

EM electron microscopy

ER endoplasmatic reticulum

FS freeze substitution

GABA $\quad \gamma$-Aminobutyric acid

GF gain-of-function

GFP green fluorescent protein

GIT G-protein coupled receptor kinase-interactor

GRIP1 glutamate receptor-interacting protein 1

GST glutathione-S-transferase

HPF high pressure freezing

HSN hermaphrodite specific neurons

INS-22 type-alpha INSulin-like molecule-22

kDa kilo Dalton

KIF1A kinesin family member $1 \mathrm{~A}$

LAR LCA-related protein tyrosine phosphatase 


\begin{tabular}{ll}
\hline LF & loss-of-function \\
LH & liprin homology \\
MA & muscle arm \\
MALS & multiangle light scattering \\
NLP-21 & neuopeptide-like protein-21 \\
NMJ & neuromuscular junction \\
NSF & N-ethylmaleimide-sensitive fusion protein \\
PH & pleckstrin homology \\
PI(4,5)P 2 & phosphatidylinositol-4,5-bisphosphate \\
Rab & Ras-related in brain \\
RIM & Rab interacting molecule \\
RPM-1 & regulator of presynaptic morphology \\
RRP & readily releasable pool \\
RSY-1 & Regulator of synaptogenesis \\
SAM & sterile alpha motif \\
SGF & supergain-of-function \\
SNAP & soluble NSF attachment proteins \\
SNAP-25 & soluble NSF attachment proteins \\
SNB & synaptobrevin \\
SNB-1 & synaptobrevin-1 \\
SV & synaptic vesicle \\
SYD-1 & synapse defective 1 \\
SYD-2 & synapse defective 2 \\
Unc & uncoordinated \\
WT & wild type \\
YFP & Yellow fluorescent protein \\
Zn & \\
zinc-finger domain \\
RN
\end{tabular}




\subsection{Solutions}

\section{Luria-Bertani (LB) Medium}

$5 \mathrm{~g} / \mathrm{l}$ Peptone (Roth)

2.5g/l Yeast extract (Roth)

$5 \mathrm{~g} / \mathrm{l} \mathrm{NaCl}$ (Roth)

$7.5 \mathrm{~g} / \mathrm{l}$ Agar (Roth), for plates

$100 \mu \mathrm{g} / \mathrm{ml}$ Ampicillin (Roth)

$15 \mu \mathrm{g} / \mathrm{ml}$ Kanamycin (Roth)

\section{Nematode Growth Medium (NGM)}

$16 \mathrm{~g} / \mathrm{l}$ Agar

$3 \mathrm{~g} / \mathrm{l}$ Peptone

$3 \mathrm{~g} / \mathrm{NaCl}$

$25 \mathrm{ml} / \mathrm{l}$ 1M KPO4 Solution (Roth)

$1 \mathrm{ml} / \mathrm{l} 1 \mathrm{M} \mathrm{MgSO} 4$ Solution (Roth)

$1 \mathrm{ml} / / 1 \mathrm{M} \mathrm{CaCl} 2$ Solution (Roth)

$1 \mathrm{ml} / \mathrm{l} 10 \mathrm{mg} / \mathrm{ml}$ Nystatin (Sigma)

$1 \mathrm{ml} / \mathrm{l} 5 \mathrm{mg} / \mathrm{ml}$ Cholesterol (Roth)

\section{M9 buffer}

$3 \mathrm{~g} \mathrm{KH}_{2} \mathrm{PO}_{4}$

$6 \mathrm{~g} \mathrm{Na}_{2} \mathrm{HPO}_{4}$

$5 \mathrm{~g} \mathrm{NaCl}$

$1 \mathrm{ml} 1 \mathrm{M} \mathrm{MgSO}_{4}$

\section{Full media YAPD (1 L)}

$10 \mathrm{~g}$ Yeast extract

$20 \mathrm{~g}$ Peptone

$20 \mathrm{~g}$ Glucose

$1 \mathrm{~g}$ Adenin sulphate

$20 \mathrm{~g}$ Agar

\section{One Step Buffer}

0,2 M Lithium actetat (Roth; 0,4 g)

40\% PEG 4000 (Sigma; 12-15 g)

$100 \mathrm{mM}$ DTT $(0,46 \mathrm{~g})$

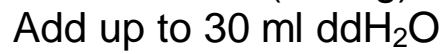

Autoclave or filtrate

Yeast minimal growth medium

$6.8 \mathrm{~g}$ Yeast nitrogen base (Invitrogen)

$1.0 \mathrm{~g} 16$ amino acid mix (-leu. -trp, -his, -ura)

In $940 \mathrm{ml}$ water

$\mathrm{pH} \mathrm{6-7}$

$20 \mathrm{~g}$ Select agar (Invitrogen)

Autoclave solution

$10 \mathrm{ml}$ amino acid solution

(+his $480 \mathrm{mg} / 200 \mathrm{ml}$ )

(+ura $480 \mathrm{mg} / 200 \mathrm{ml}$ ) 
$40 \mathrm{ml} 50 \%$ Glucose

Homogenization buffer

20mMHEPES pH 7.4

$10 \mathrm{mM} \mathrm{KCl}$

$1 \mathrm{mM}$ EDTA

400 mM Pefabloc (Roche)

$10 \%$ APS solution

$1 \mathrm{~g}$ APS (Roth)

$10 \mathrm{ml} \mathrm{ddH} 2 \mathrm{O}$

2x SDS sample buffer

$121.1 \mathrm{~g} / \mathrm{l}$ Tris $\mathrm{pH} 6.8$

$20 \%$ Glycerol (v/v)

$100 \mathrm{~g} / \mathrm{l} \mathrm{SDS}$

$0.002 \%$ Bromophenol blue (w/v)

$200 \mathrm{ml}$ 2-Mercaptoethanol (Sigma Aldrich)

\section{5\% Stacking gel}

$7.5 \mathrm{ml} 30 \%$ Acrylamide/Bis (Roth)

$14.8 \mathrm{ml} \mathrm{ddH} 2 \mathrm{O}$

$7.5 \mathrm{ml} 1.0 \mathrm{M}$ Tris, pH 6.8

$300 \mu \mathrm{l} 10 \%$ SDS

$200 \mu \mathrm{l} 10 \%$ APS (Roth)

$10 \mu \mathrm{l}$ TEMED (Roth)

Separating Gel - 20ml

$2.6 \mathrm{ml} 30 \%$ Acrylamide/Bis

$12.4 \mathrm{ml} \mathrm{ddH} 2 \mathrm{O}$

$5 \mathrm{ml} 1.5 \mathrm{M}$ Tris, pH 8.8

$200 \mu \mathrm{l} 10 \%$ SDS

$400 \mu \mathrm{l} 10 \%$ APS

$20 \mu \mathrm{I}$ TEMED

SDS running buffer (10x)

$30.3 \mathrm{~g} / \mathrm{l}$ Tris

$144 \mathrm{~g} / \mathrm{l}$ Glycine (Roth)

$10 \mathrm{~g} / \mathrm{l} \mathrm{SDS}$ (Roth)

Semi Dry Blot buffer (2 Liter)

11,6 g TRIS

5,8 g Glycin

$0,74 \mathrm{~g}$ SDS

$400 \mathrm{ml} \mathrm{MeOH}$

$\mathrm{pH} 8,9$

\section{1x TBST 1 Liter}

$10 \mathrm{mM}$ TRIS-HCl pH 8,0

$0.05 \%$ Tween 20

$150 \mathrm{mM} \mathrm{NaCl}$ 
Western blot blocking buffer:

$4 \%$ skim milk powder (Sucofin) in 1xTBST

Western blot developing solution

$1 \mathrm{ml}$ Solution A

$100 \mu$ Solution B

$3 \mu \mathrm{l} \mathrm{H}_{2} \mathrm{O}_{2}$

\section{Solution A}

$200 \mathrm{ml} \mathrm{0.1M}$ Tris $\mathrm{HCl}(\mathrm{pH} 8.6)$

50 mg Luminol (Sigma)

\section{Solution B}

$11 \mathrm{mg}$ para-hydroxycoumarin acid (dilute in $10 \mathrm{ml}$ DMSO)

$35 \% \mathrm{H} 2 \mathrm{O} 2$

\section{X PCR buffer}

$100 \mathrm{mM}$ Tris $\mathrm{HCl}$

$500 \mathrm{mM} \mathrm{KCl}$

$30 \mathrm{mM} \mathrm{MgCl} 2$

$\mathrm{pH} 8,3$

$1,49 \mathrm{~g} \mathrm{KCl}$

$0,076 \mathrm{~g} \mathrm{MgCl}_{2}$

$4 \mathrm{ml} 1 \mathrm{M}$ Tris $\mathrm{HCl}$

Add $\mathrm{H}_{2} \mathrm{O}$ to $40 \mathrm{ml}$ total

\subsection{Primer}

Table 5 DNA sequences of all primers used in this study. OGQ numbers refer to an internal reference number of the Eimer lab. Primers are designed for $C$. elegans genome or cDNA libraries. Forward primer $=\mathrm{fwd}$; reverse primer $=\mathrm{rev}$

\begin{tabular}{lll} 
Primer & Primer sequence & Description \\
\hline oGQ 1101 & TTGCATCTGCAAAAGAAACG & $\begin{array}{l}\text { Fwd; outside primer to detect syd- } \\
\text { 2(ok217) deletion }\end{array}$ \\
\hline oGQ 1102 & GCTCCGAACGAAAGAAGTTG & $\begin{array}{l}\text { Rev; outside primer to detect syd-2(ok217) } \\
\text { deletion }\end{array}$ \\
\hline oGQ 2379 & TTTCACGAGCATCTTCTGTC & Fwd; within syd-2(ok217) deletion \\
\hline oGQ 2452 & CAACAACCAGCTCAACAGTATC & Rev; within syd-2(ok217) deletion \\
\hline oGQ 1417 & AGATGAAGAGATTGTCGAG & Fwd; sequencing primers for syd-2(ju487) \\
& & point mutation \\
\hline oGQ 1418 & GGTATGTGATGTATTCTCG & Rev; sequencing primers for syd-2(ju487) \\
& & point mutation \\
\hline oGQ 1419 & CACAACGCACCGATCA & Fwd; sequencing primers for syd-2(ju37) \\
& & point mutation
\end{tabular}


Appendix

\begin{tabular}{|c|c|c|}
\hline oGQ 1420 & CTTGCATTCGTTCTTTGA & $\begin{array}{l}\text { Rev; sequencing primers for syd-2(ju37) } \\
\text { point mutation }\end{array}$ \\
\hline oGQ 1904 & CTCACCACAGCTTTAGAATCCG & $\begin{array}{l}\text { Fwd; outside primer to detect elks-1(js816) } \\
\text { deletion }\end{array}$ \\
\hline oGQ 1905 & CTCACCGCTTCTTTGCTTGCAC & $\begin{array}{l}\text { Rev; outside primer to detect elks-1(js816) } \\
\text { deletion (partially intron!) }\end{array}$ \\
\hline oGQ 2037 & CTTGCACAAGATCCCGATTC & $\begin{array}{l}\text { Rev; outside primer to detect elks- } \\
\text { 1(ok2762) deletion }\end{array}$ \\
\hline oGQ 2038 & $\begin{array}{l}\text { CCCCCACCGGTAATGGCACCTGGTCC } \\
\text { CGCACCA }\end{array}$ & $\begin{array}{l}\text { Fwd; introduces a Agel site in front of the } \\
\text { elks-1 ATG when amplified from cDNA }\end{array}$ \\
\hline oGQ 2040 & GGAGCAACAGAAGACCTATCAG & $\begin{array}{l}\text { Fwd; additional sequencing primer for } \\
\text { C. elegans elks-1 }\end{array}$ \\
\hline oGQ 2056 & GCGGTCTGCATTCTTGTCTG & $\begin{array}{l}\text { Rev; primer behind BamH1 site to amplify } \\
\text { first part of elks-1 with fwd primer oGQ } \\
2038\end{array}$ \\
\hline oGQ 2063 & CGAATGGAACTCCGAGAACG & $\begin{array}{l}\text { Fwd; outside primer to detect elks-1 } \\
\text { (ok2762) deletion and amplify second } \\
\text { piece of elks-1 }\end{array}$ \\
\hline oGQ 2068 & $\begin{array}{l}\text { CCCCTCGAGTCAGCGGCCGCCGGCC } \\
\text { CAAATTCCGTCAGCATC }\end{array}$ & $\begin{array}{l}\text { Rev; to amplify second part of elks-1 } \\
\text { removing the STOP and introducing } \\
\text { instead a Notl, a STOP and a Xhol site }\end{array}$ \\
\hline oGQ 2150 & CCGGGCCTCTTTGTTCATTG & $\begin{array}{l}\text { Fwd; sequencing primer to detect base } \\
\text { exchange in rsy-1(wy94) mutants }\end{array}$ \\
\hline oGQ 2151 & TGGACCAACTATCATCAGGCAC & $\begin{array}{l}\text { Rev; sequencing primer to detect base } \\
\text { exchange in rsy-1(wy94) mutants }\end{array}$ \\
\hline oGQ 2181 & $\begin{array}{l}\text { GTTCGTGAATGTCTTCGAGTAGCAATG } \\
\text { GAGCGT }\end{array}$ & $\begin{array}{l}\text { Fwd; for PCR mutagenesis to introduce } \\
\text { syd-2(ju487) point mutation in LH1 domain }\end{array}$ \\
\hline oGQ 2182 & $\begin{array}{l}\text { TACTCGAAGACATTCACGAACTTTTTCA } \\
\text { TCCAA }\end{array}$ & $\begin{array}{l}\text { Rev; for PCR mutagenesis to introduce } \\
\text { syd-2(ju487) point mutation in LH1 domain }\end{array}$ \\
\hline oGQ 2183 & $\begin{array}{l}\text { CCCCATATGGCACCGGTACAGGAACTT } \\
\text { CAAACAATGACG }\end{array}$ & $\begin{array}{l}\text { Fwd; primer introducing a Ndel and Agel } \\
\text { restriction site at start of the syd-2 LH1 } \\
\text { domain }\end{array}$ \\
\hline oGQ 2237 & $\begin{array}{l}\text { CCCCTCGAGTCAGCGGCCGCCCGAAT } \\
\text { TCTCATCTCСTTTTGT }\end{array}$ & $\begin{array}{l}\text { Rev; primer introducing a Notl, a STOP } \\
\text { and a Xhol restriction site at the end of the } \\
\text { syd-2 LH1 domain }\end{array}$ \\
\hline oGQ 2495 & CGCCСTCTTTCTCTTTCCTTAC & $\begin{array}{l}\text { Fwd; outside primer to detect } \\
\text { unc-10(md1117) deletion }\end{array}$ \\
\hline oGQ 2496 & CCGAGACAGAGGACTCACAC & $\begin{array}{l}\text { Rev; inside primer to detect unc- } \\
\text { 10(md1117) deletion }\end{array}$ \\
\hline
\end{tabular}


Appendix

oGQ 2497 AGCCTACTAACCCATTTCTCACTG Rev; outside primer to detect

unc-10(md1117) deletion

OGQ 2498 TGCCAAAGCAAAGACAAACG

Fwd; inside primer to detect unc-

10(md1117) deletion 


\section{Curriculum vitae}

\section{Personal Information}

Name: Maike Kittelmann, née Hartisch

Date of birth: $25^{\text {th }}$ of November, 1983 in Erfurt, Germany

Nationality: German

Private Address: Kaakweg 15

37077 Goettingen

Email: m.kittelmann@eni-g.de

Working address: European Neuroscience Institute Goettingen, Molecular Neurogenetics

Grisebachstrasse 5, 37077 Goettingen

\section{Education}

02/2009 to date:

PhD Thesis

European Neuroscience Institute Göttingen, Germany

"Synaptic Ultrastructure and Regulation of Synaptic

Transmission in Caenorhabditis elegans"

Supervisor: Dr. Stefan Eimer

Göttingen Graduate School for Neurosciences, Biophysics, and Molecular Biosciences (GGNB)

Sensory and Motor Neuroscience Doctoral Program

Partially funded by GGNB Junior Group Stipend.

04/2008 to 01/2009: Diploma Thesis

Max Planck Institute for Experimental Medicine, Göttingen

"Morphological analysis of the secretion apparatus of

Trichoplax adhaerens"

Supervisor: Dr. Frederique Varoqueaux

Final degree: sehr gut $(A)$

10/2003 to 01/2009: Undergraduate Studies of Biology

Georg-August-University of Göttingen; Germany

\section{Fellowships, Awards}

2009/2010: Education Abroad Program scholarship for studies abroad at the University of California San Diego (living expenses and tuition waiver)

2009/2010: Fulbright Scholarship Travel Grant for studies abroad at University of California San Diego

01/2010 to 12/2012: Junior Group stipend GGNB Göttingen 
09/ 2009 to 07/2010: Studies abroad as Education Abroad Program (EAP) and Fulbright scholar at the University of California, San Diego, USA

Collaborator and host: Dr. Yishi Jin Electron Microscopy-Collaborator: Mark Ellisman

08/2000 to 06/2001: Exchange Student with Youth for Understanding (YFU) in Maquoketa, lowa, USA

\section{Teaching Experiences}

11/2008 to 01/2009: Supervision of lab rotation student Cordelia Imig "Morphology of Monosiga brevicollis"

$25^{\text {th }}-29^{\text {th }}$ July 2011: Organizer and Tutor of the Workshop and Symposium "High Pressure Freeze Electron Microscopy on its way to Correlative Microscopy and 4D EM"

11/2008 to 01/2009: Supervision of lab rotation student Cordelia Imig "Morphology of Monosiga brevicollis"

$12 / 2011$ to $02 / 2012$ Supervision of master lab rotation student David Vasmer "Mitochondrial toxicity of $\alpha$-Synuclein oligomers in C. elegans

\section{Work experience}

05 and 06/2006: Student assistant for Daniela Gocht, Zoology/Anthropology Department for Neurobiology, University of Göttingen

05/2007 to 05/2008: Student assistant for Nikola-Michael Prpic, Zoology/Anthropology, Department for Developmental Biology, University of Göttingen

\section{Publications}

Kittelmann, Hegermann, Goncharov, Taru, Dai, Richmond, Ellisman, Jin, Eimer. Liprin- $\alpha / S Y D-2$ determines the size of active zone dense projection in C. elegans. Manuscript in preparation

Chua, Butkevich, Worseck, Kittelmann, Grønborg, Behrmann, Stelzl, Pavlos, Lalowski, Eimer, Wanker, Klopfenstein, Jahn (2012). Phosphorylation-regulated axonal dependent transport of syntaxin 1 is mediated by a Kinesin-1 adapter. Proc Natl Acad Sci U S A. 109(15):5862-7.

Kittelmann, Schinko, Winkler, Bucher, Wimmer, Prpic. Insertional mutagenesis screening identifies the zinc finger homeodomain 2 (zfh2) gene as a novel factor required for embryonic leg development in Tribolium castaneum (2009). Dev Genes Evol. 219(8):399-407. 


\section{Oral presentations}

02/2009: Frühjahrstreffen des Arbeitskreises PANOS der Deutschen

Gesellschaft für Elektronenmikroskopie

Talk title: "Morphological analysis of the secretion apparatus of

Trichoplax adhaerens"

03/2011: Spring--Meeting of PANOS working group on "Correlative Microscopy" at MPI Dortmund

Talk title: Synaptic Ultrastructure in C. elegans

09/ 2011: Ribbon Synapses Symposium 2011, Göttingen

Talk title: Synaptic Ultrastructure in C. elegans

\section{Conferences and Poster presentations}

02/2009: PANOS, Münster

03/2009: $\quad 8^{\text {th }}$ Göttingen Meeting of the German Neuroscience Society

06/2010: Gordon Research Conference, New Hampshire

03/2011: $\quad$ PANOS, Dortmund

05/2011: Neurizons, Göttingen

09/2011: Ribbon Synapse Symposium, Göttingen 